\title{
IntechOpen
}

\section{Advances in \\ Rehabilitation of Hearing Loss}

Edited by Diego Zanetti and Federica Di Berardino
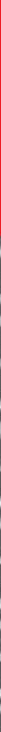



\section{Advances in Rehabilitation of Hearing Loss}

Edited by Diego Zanetti and Federica Di Berardino 

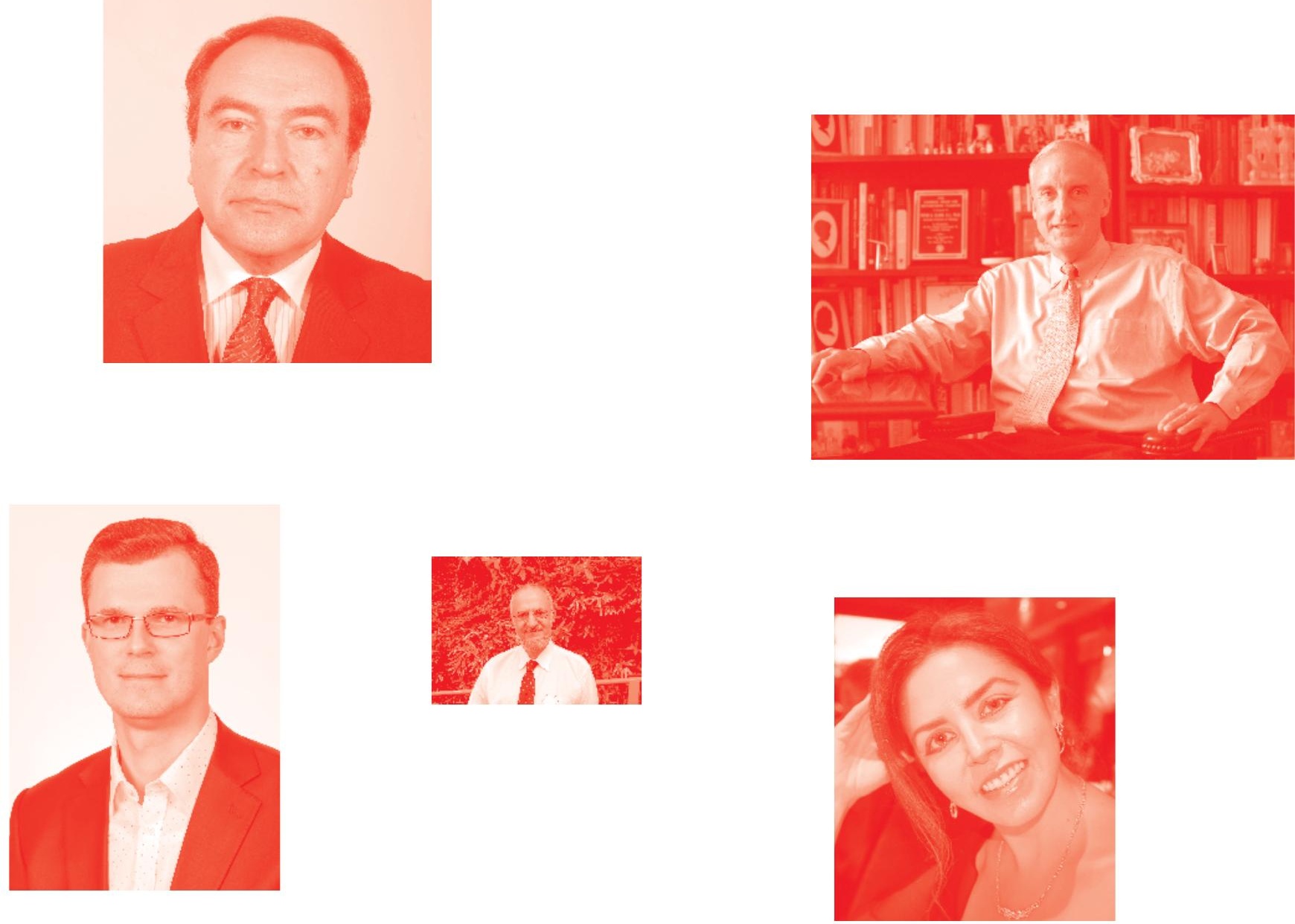

Supporting open minds since 2005
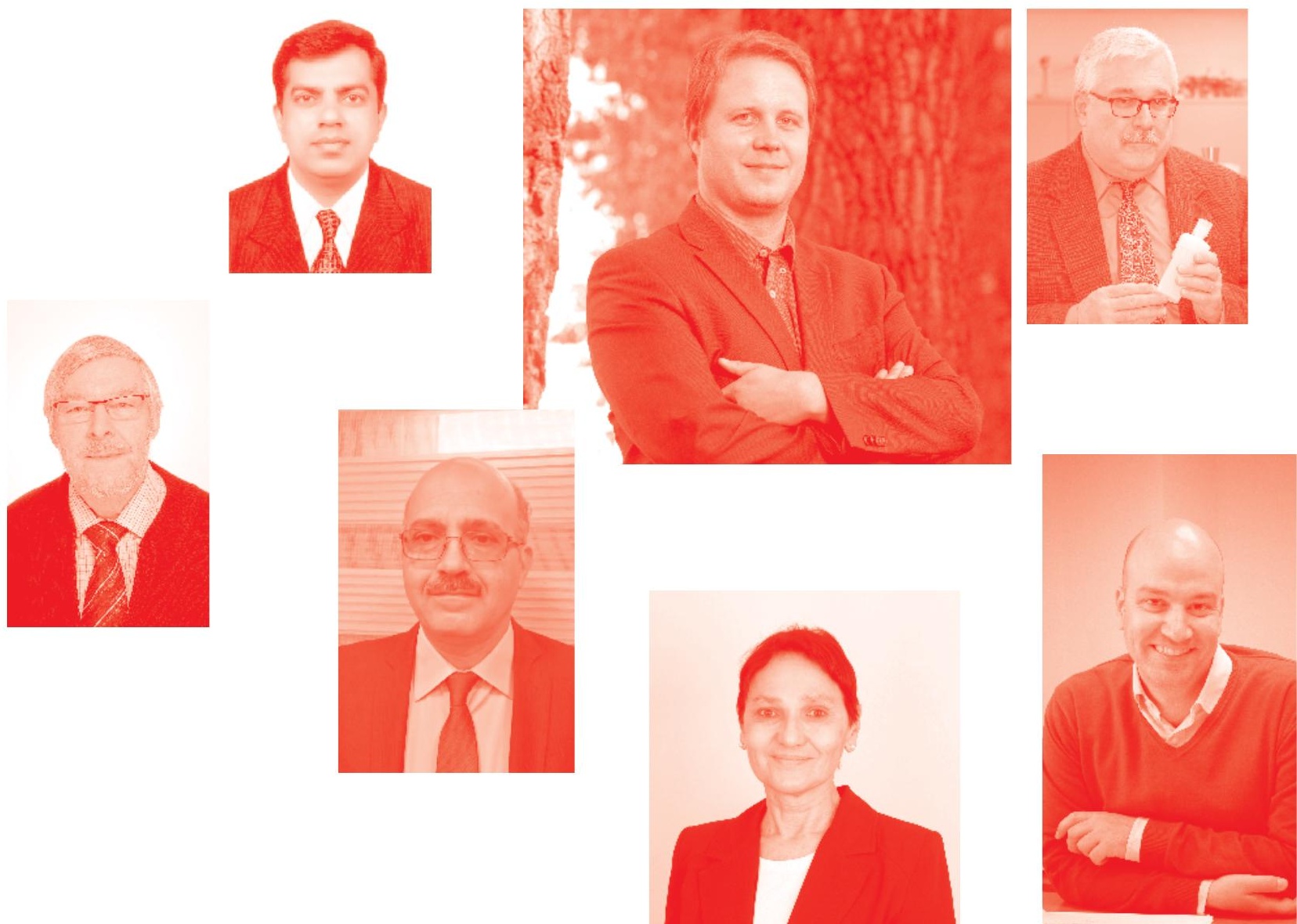
Advances in Rehabilitation of Hearing Loss

http : //dx . doi.org/10.5772/intechopen. 80097

Edited by Diego Zanetti and Federica Di Berardino

\section{Contributors}

Hajime Sano, James Baumgartner, Linda Baumgartner, Ernest Moore, Steven Messina, Michael Seidman, David Shook, Deborah Lamontagne, Brian Taylor, Yasser Fouad, Maurizio Barbara, Simonetta Monini, Patrick S. C. D'Haese, Vincent Van Rompaey, Marc De Bodt, Paul Van De Heyning, Federica Di Berardino, Giorgio Conte, Diego Zanetti, Silvia Casale, Sara Sbaraini, Giampietro Ricci, Arianna Di Stadio, Antonio Della Volpe, Valeria Gambacorta, Angel Ramos-Macias, Silvia Borkoski Barreiro, Juan Carlos Falcón González, Ángel Ramos de Miguel, Bernard Fraysse, Chris J. James

( ) The Editor(s) and the Author(s) 2020

The rights of the editor(s) and the author(s) have been asserted in accordance with the Copyright, Designs and Patents Act 1988. All rights to the book as a whole are reserved by INTECHOPEN LIMITED. The book as a whole (compilation) cannot be reproduced, distributed or used for commercial or non-commercial purposes without INTECHOPEN LIMITED's written permission. Enquiries concerning the use of the book should be directed to INTECHOPEN LIMITED rights and permissions department (permissions@intechopen.com).

Violations are liable to prosecution under the governing Copyright Law .

\section{(cc) BY}

Individual chapters of this publication are distributed under the terms of the Creative Commons Attribution 3.๑ Unported License which permits commercial use, distribution and reproduction of the individual chapters, provided the original author(s) and source publication are appropriately acknowledged. If so indicated, certain images may not be included under the Creative Commons license. In such cases users will need to obtain permission from the license holder to reproduce the material. More details and guidelines concerning content reuse and adaptation can be found at http : //www . intechopen . com/copyright-policy . html .

\section{Notice}

Statements and opinions expressed in the chapters are these of the individual contributors and not necessarily those of the editors or publisher. No responsibility is accepted for the accuracy of information contained in the published chapters. The publisher assumes no responsibility for any damage or injury to persons or property arising out of the use of any materials, instructions, methods or ideas contained in the book.

First published in London, United Kingdom, 2020 by IntechOpen IntechOpen is the global imprint of INTECHOPEN LIMITED, registered in England and Wales, registration number: 11086078 , 7th floor, 10 Lower Thames Street, London,

EC3R 6AF, United Kingdom

Printed in Croatia

British Library Cataloguing-in-Publication Data

A catalogue record for this book is available from the British Library

Additional hard and PDF copies can be obtained from orders@intechopen.com

Advances in Rehabilitation of Hearing Loss

Edited by Diego Zanetti and Federica Di Berardino

p. $\mathrm{cm}$.

Print ISBN 978-1-78985-249-3

Online ISBN 978-1-78985-250-9

eBook (PDF) ISBN 978-1-83880-481-7 


\section{We are IntechOpen, \\ the world's leading publisher of Open Access books}

Built by scientists, for scientists

\section{$4,700+$}

Open access books available

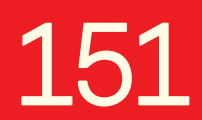

Countries delivered to

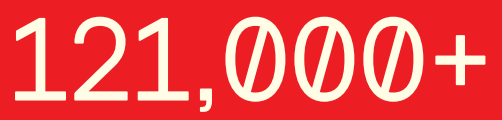

International authors and editors

Our authors are among the

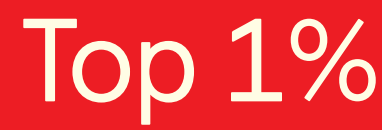

most cited scientists

Contributors from top 500 universities
40010

Downloads

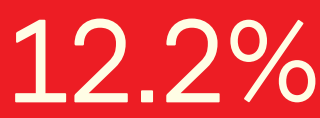

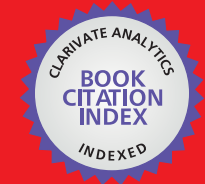

WEB OF SCIENCE ${ }^{\text {MM }}$

Selection of our books indexed in the Book Citation Index in Web of Science ${ }^{\mathrm{TM}}$ Core Collection (BKCI)

Interested in publishing with us?

Contact book.department@intechopen.com

Numbers displayed above are based on latest data collected.

For more information visit www.intechopen.com

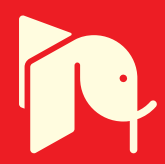





\section{Meet the editors}

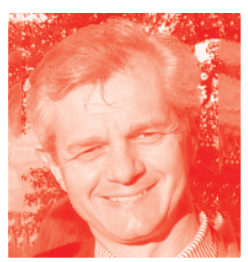

Dr Zanetti has been an otologic and skull base surgeon at the University of Milan since 2015. He worked in otologic surgery and hearing implants at the ENT Dept of the University of Brescia (Italy) (1986-2010) and at the ENT Department of the University of Milano Bicocca (Italy) (2010-2014). He was the Head of the Cochlear Implants Program at the Audiology Department of the IRCCS Fondazione Ca 'Granda Hospital (2015-2019). He graduated in medicine in July 1983. In 1986, he specialized in otolaryngology - head and neck surgery at the University of Milano. In 1995, he specialized in audiology at the University of Padua (Italy). He has undertaken training fellowships at the House Ear Institute, Los Angeles (USA), Instituto de Otologia Garcia-Ibanez, Barcelona (Spain), and Fondation Piortmann, Bordeaux (France). He is the author of 90 publications in international scientific journals. He is the co-author of seven chapters of books and editor of one book on "Cochlear Implants". He is a member of EAONO, Politzer, EFAS, ESBS, Charles Bell, Meniere Societies, Italian Society of Audiology and Phoniatrics, and of the Italian Society of Otolaryngology. His current research interest is focused on cochlear implants and rehabilitative surgery for deafness.

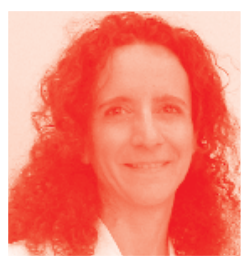

Dr Berardino has been a Professor of Audiology at the University of Milan since 2011. She was a clinical assistant at the Audiology Department of the IRCCS Fondazione Ca 'Granda Hospital since 2009. She graduated in medicine in July 1999. On November 2005, she specialized in audiology and phoniatrics. From September 2006 to-August 2009, she was a Rresearch postdoctoral Fellow (3 years) on "Deafness and cognitive and motor decline". She is the a Author of 70 publiations on in national andinternational relevant ournals. She is the co-member of the EFAS (European federation of Audiological Societies), Italian Society of Audiology and Phoniatrics, and Italian Society of Otolaryngology. Her research interest is the study of etiological factors in audiological and vestibular diseases and in the development and clinical validation of new tools for diagnostic purposes. 



\section{Contents}

Preface

Section 1

Research and Clinical Science

Chapter 1

Associated Health Issues of Patients with Acquired Unilateral

Hearing Loss

by Hajime Sano

Chapter 2

The Site of Lesion in Hearing Loss: Advances in Otoneuroradiology by Giorgio Conte, Silvia Casale, Sara Sbaraini, Federica Di Berardino and Diego Zanetti

Chapter 3

Audiology's Third Pillar: Comprehensive Follow-Up Care and Counseling for Those Who Choose to Self-Direct Their Care

by Brian Taylor

Chapter 4

Progenitor Cell Therapy for Sensorineural Hearing Loss in Infants

by Linda Baumgartner, Michael Seidman, Deborah Lamontagne,

Ernest Moore, David Shook, Steven Messina and James Baumgartner

Section 2

Implantable Hearing Aids

Chapter 5

Congenital Aural Atresia: Hearing Rehabilitation by Bone-Anchored

Hearing Implant (BAHI)

by Giampietro Ricci, Arianna Di Stadio, Valeria Gambacorta

and Antonio Della Volpe

Chapter 6

The Esteem ${ }^{\circledR}$, Fully Implantable Middle Ear Device

by Maurizio Barbara and Simonetta Monini 
Section 3

Cochlear Implants

Chapter 7

The Need to Increase Awareness and Access to Cochlear Implantation by Patrick S.C. D'Haese, Vincent Van Rompaey, Marc De Bodt and Paul Van de Heyning

Chapter 8

Advances in Surgical and Anesthetic Techniques for Cochlear Implantation

by Yasser A. Fouad

Chapter 9

Prognostics Factors of Cochlear Implant in Adults: How Can We Improve Poorer Performers?

by Bernard Fraysse and Chris J. James

Chapter 10

Cochlear Implant in Single-Sided Deafness Children and Adults by Ángel Ramos Macías, Silvia A. Borkoski Barreiro,

Juan Carlos Falcón González and Ángel Ramos de Miguel 


\section{Preface}

Severe hearing loss (HL) can have a significant negative impact on communication, social interactions, and emotional well-being. The WHO estimates that there are about 360 million people in the world with disabling hearing loss $(5.3 \%$ of the world's population). Ninety-one percent of these are adults and $9 \%$ are children.

The last decades have witnessed impressive technological advances in the rehabilitation of HL, leading to significant shifts in the approach and protocols.

The majority of HL cases are of the sensorineural type and can be managed by amplification with hearing aids or active middle ear hearing implants.

In profound $\mathrm{HL}$, the speech perception performance achieved with cochlear implants has been so reliable and rewarding that it has enabled a great expansion of their audiological indication. Bone conduction hearing implants, instead, allow a consistent correction of conductive or mixed hearing losses.

Stem cell manipulation and genetic therapy appear promising but are not yet available for clinical application.

This book strives to provide an in-depth overview of the latest developments in the rehabilitation of HL, through contributions by international leading experts, focused on specific topics within the boundaries of this continuously evolving area.

Hopefully, the insights will assist clinicians, audiologists, hearing aid acousticians, and speech therapists in their planning of the most suitable treatment options for their patients with severe-to-profound hearing loss.

The editor wishes to acknowledge and congratulate his co-editor and all authors for their excellent scientific contributions; Sara Cavicchiolo (Sp. Ther.), Anna Gasbarre (Aud. Tech.), Eliana Filipponi (Aud. Tech.), Cinzia Lazzarini (Sp. Ther.), and Loredana Todini (Sp. Ther.), from the team of the Audiology Dept. of the University of Milano for their trusted cooperation; and Associazione Progetto Udire Onlus for their continuous support.

Diego Zanetti and Federica Di Berardino Fondazione IRCCS Cà Granda, Ospedale Maggiore Policlinico, Università degli Studi di Milano, Milan, Italy 

Section 1

\section{Research and Clinical Science}





\title{
Associated Health Issues of Patients with Acquired Unilateral Hearing Loss
}

\author{
Hajime Sano
}

\begin{abstract}
Patients with unilateral hearing loss have impaired hearing of sounds coming from the affected side, decreased comprehension of speech in noisy environments, and lack of sound localization. There are many conditions that can induce unilateral hearing loss, but idiopathic sudden sensorineural hearing loss (ISSHL) is thought to be most notable because of its high incidence. Patients with ISSHL suddenly acquire unilateral hearing loss; therefore, there are additional significant health problems that complicate the abovementioned symptoms due to the characteristic clinical course of ISSHL including hearing-related discomfort, tinnitus, and anxiety. It has been reported that hearing-related discomfort is closely associated with patients' quality of life. In this chapter, the associated health issues of patients with ISSHL are described, and the interventions employed for patients with unilateral hearing loss are evaluated for their potential in improving the lives of ISSHL patients.
\end{abstract}

Keywords: idiopathic sudden sensorineural hearing loss, bone-anchored hearing aids, contralateral routing of signals, cochlear implant, hearing-related discomfort

\section{Introduction}

Because individuals with unilateral hearing loss have normal hearing in the opposite ear, impairment of auditory communication is less severe than in those with bilateral hearing loss. Therefore, interventions for these patients may not always be indicated. Unilateral hearing loss that is profound is known as singlesided deafness (SSD). Patients with SSD suffer from impaired hearing of sounds coming from the deaf side, lack of sound localization, and deteriorated comprehension of speech in noisy environments. These disorders and related problems can affect academic performance in children. Kuppler et al. reviewed that some children with SSD have significantly decreased self-esteem and increased level of exhaustion and stress because of the effort required to hear, and the tenfold increase of incidence (35\%) of poor performer will be estimated [1]. It was also inferred that SSD have adverse effects on quality of life (QOL) and social life of both children and adults.

The causes of unilateral hearing loss include congenital and acquired diseases. Diseases inducing acquired unilateral hearing loss include idiopathic sudden sensorineural hearing loss (ISSHL), Meniere's disease, mumps, vestibular schwannoma, otosclerosis, otitis media with effusion, chronic otitis media, and cholesteatoma, among others. Hearing loss caused by otosclerosis, chronic otitis media, and 
cholesteatoma can be improved by surgery, but inner ear damage resulting in SSD can rarely be reversed. Meniere's disease usually presents with mild-to-moderate hearing loss. Vestibular schwannoma presents with a wide range of hearing loss, from normal to total deafness, and if the tumor grows, surgical treatment is required, but the possibility of inducing SSD is relatively high after surgery. Though mumps is a disease that can acutely cause SSD, the incidence of hearing complications in mumps patients is quite low. ISSHL is quite a common condition, and it is estimated to produce the most patients with irreversible unilateral hearing loss.

Patients with ISSHL have an extremely dramatic clinical course in which unilateral hearing loss suddenly develops from normal hearing on both sides. In addition to the hearing-related problems that patients with congenital unilateral hearing loss or gradually worsening unilateral hearing loss face, there are other subsequent health issues that arise; therefore, ISSHL could be considered the most significant cause of acquired unilateral hearing loss. This chapter focuses on ISSHL as a representative condition that induces acquired unilateral hearing loss. First, the symptoms and health issues of patients with ISSHL based on the results of a nationwide survey of patients with ISSHL in Japan will be described. Next, the therapeutic interventions for patients with irreversible unilateral hearing loss caused by ISSHL will be considered.

\section{Epidemiology and frequency of ISSHL}

ISSHL is the sudden or acute onset of sensorineural hearing loss of unknown origin. The hearing loss is unilateral in most cases with bilateral involvement reported in $<5 \%$ of cases [2]. The lesion is most often cochlear in origin, and less frequently retrocochlear. Although the cause of ISSHL has not been identified, several pathogenic possibilities have been proposed, such as vascular disorders, viral infections, and membrane breaks. Many treatment regimens have been investigated, including corticosteroids, vasoactive drugs, antiviral drugs, and hyperbaric oxygenation therapy, but none have proven effective. ISSHL is expected to improve on its own or with treatment; however, hearing levels become fixed $\sim 2$ months from the onset, and if it is not cured by then, permanent hearing loss remains.

The incidence of ISSHL is reported to be 3-30 per 100,000 population per year [2], but a recent report in Japan reported 60 per 100,000 population per year [3]. According to the population of Japan, about 78,000 new cases occur annually. For the hearing performance of 1113 patients with ISSHL in the author's hospital during the past 20 years during the persistent phase after treatment, $35 \%$ were completely cured, $27 \%$ had mild hearing loss, $26 \%$ had moderate hearing loss, $8 \%$ had severe hearing loss, and $4 \%$ had profound to total deafness. When this is considered with the above-estimated incidence, it is estimated that 21,000 patients with mild hearing loss, 20,000 patients with moderate hearing loss, 6200 patients with severe hearing loss, and 3100 patients with profound hearing loss develop unilateral hearing loss each year in Japan. Although vestibular schwannoma is also a relatively common disease inducing SSD, the number of surgeries performed annually in Japan to remove the tumors is estimated to be approximately $700[4,5]$, considerably less than the number of patients with SSD caused by ISSHL.

\section{Symptoms and QOL in patients with ISSHL}

Not many reports have investigated the symptoms or QOL in patients with ISSHL. Chiossoene-Kerdel et al. used the Hearing Handicap Inventory for Adults to 
investigate the degree of handicap in patients with ISSHL [6], and most patients had a handicap associated with hearing loss and tinnitus. Carlsson et al. investigated QOL in patients with ISSHL using the EuroQoL-5D, Problems Impact Rating Scale, and the Hospital Anxiety and Depression Scale [7]. They reported that all three indicators were significantly influenced by the presence of tinnitus and vertigo. However, patients with Meniere's disease may have been included in that report, because the incidence of vertigo at the time of investigation was quite high (34\%). Two main symptoms were reported to affect QOL in ISSHL patients with persistent hearing problems: difficulty in hearing and tinnitus $[6,7]$. Unilateral hearing loss is a sudden change for patients who have never experienced hearing problems before the onset of ISSHL. Other problems, such as hearing-related discomfort and anxiety about recurrence, may also affect QOL. These problems may differ from those in patients with congenital SSD.

I and a few others conducted a multicenter clinical study by the Acute Profound Deafness Research Committee of the Ministry of Health, Labour and Welfare in Japan to investigate the symptoms and QOL in the patients with ISSHL in their persistent phase $[8,9]$. The results from that study are described below.

A total of 140 patients with ISSHL (64 males, 76 females; mean age 59.1 years; range $21-85$ years) and 24 patients with congenital SSD (13 males, 11 females; mean age 30.5 years; range 20-77 years) were investigated to determine their symptoms. In the patients with ISSHL, hearing levels of the affected ear were widely distributed from normal to profound, with a peak distribution of $70 \mathrm{dBHL}$. The distribution of time intervals from the onset of hearing loss varied widely from 30 days to 62 years (mean 5.5 years; median 2.7 years). The majority of patients with congenital SSD (71\%) were between 20 and 29 years of age [8]. A symptom questionnaire was newly created to assess the patients' symptoms. In a previous investigation, information on symptoms was elicited from 104 patients with ISSHL using a freewriting method. These symptoms were rewritten and organized to create a new questionnaire comprising 17 questions that covered seven categories: hearing difficulty (three questions), spatial hearing (two questions), hearingrelated discomfort (four questions), tinnitus (two questions), vertigo (one question), attitude to communications (two questions), and anxiety (three questions). We also asked patients with congenital SSD to answer the same questionnaire and compared the results with those patients with ISSHL [8]. The results of four major hearing-related symptoms, hearing difficulty, disability of spatial hearing, discomfort, and tinnitus, are shown in Figure 1. In response to questions regarding hearing difficulty, patients in both groups reported that they frequently had problems. With regard to the items "conversation with several people" and "conversation in noisy place," significantly more patients with ISSHL than with congenital SSD reported difficulty in hearing. In response to all questions regarding hearing-related discomfort, significantly more patients with ISSHL than with congenital SSD experienced symptoms. With respect to tinnitus, few patients with congenital SSD and many patients with ISSHL reported this symptom. In response to questions regarding spatial hearing, no difference between the groups was identified.

The health-related QOL in the patients with ISSHL and congenital SSD was investigated using the short-form health survey version 2 (SF-36). SF-36 provides scores for eight health-related QOL domains and two more comprehensive scores: the physical component summary (PCS) and the mental component summary (MCS). The scores for the eight domains and the two component summaries were standardized (norm-based scoring, Japanese average of 50, standard deviation of 10) for comparison with the scores of people in the general population or those reported in other studies. When the average scores for the two summary components in patients with ISSHL and those with congenital 


\section{Four major symptoms in ISSHL patients}

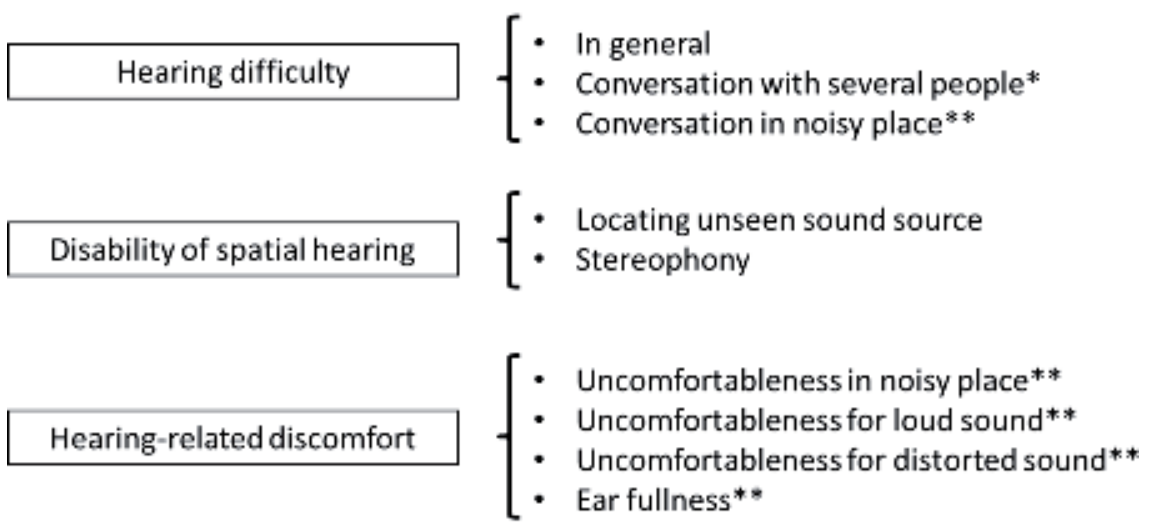

Tinnitus**

Figure 1.

Four major categories of symptoms reported by patients with ISSHL and congenital SSD. This figure was created from the results of Sano et al. [8]. "Hearing difficulty" consists of three items, "disability of spatial hearing" consists of two items, and "hearing-related discomfort" consists of four items in the questionnaire. $P$-values are presented as a comparison of the incidence between ISSHL and congenital SSD: ${ }^{*} p<0.05$, ${ }^{* *} p<0.01$.

SSD were compared with average Japanese scores matched by decade of age, the PCS scores in all age groups for both ISSHL and congenital SSD patients were not significantly different from the Japanese average scores, and MCS scores for patients in their 20s for both ISSHL and congenital SSD and in their 30s for ISSHL were not significantly different from Japanese average scores. However, the MCS scores for patients with ISSHL in their 40-70s were significantly lower than the age-matched average Japanese scores (Figure 2).

Next, multiple linear regression analysis was used to investigate confounders influencing MCS scores in patients with ISSHL. The effects of age, hearing level at the time of investigation, time from onset, and responses to three items of the symptom questionnaire (hearing difficulty in general, discomfort in noisy places, and tinnitus) were evaluated. Unexpectedly, the results showed that the response to the item of discomfort in noisy places was the sole significant confounder.

"Hearing-related discomfort" was not a particularly notable symptom before this study; therefore, we further investigated this finding. When the relationship between the response to the item "discomfort in noisy places" and the hearing level in the affected ear was investigated, the response was not associated with hearing level [8]. A high incidence of this symptom was reported by ISSHL patients with moderate to profound hearing loss in the affected ear. We can infer that people with moderate hearing loss feel discomfort from noise because they hear noisy sounds in the affected ear, which can be too loud as a result of recruitment phenomenon and can be distorted by impairment of frequency selectivity. But why do patients with profound hearing loss in their affected ear feel this discomfort? In the direct expression of the discomfort by the patients, some of them described it as "It feels very noisy because the noise around me spreads all over the space around me." We proposed that the reason for this symptom in patients with unilateral profound hearing loss was sudden loss of the ability to localize the sounds coming from various directions, and we called this condition "collapse of spatial hearing perception." 


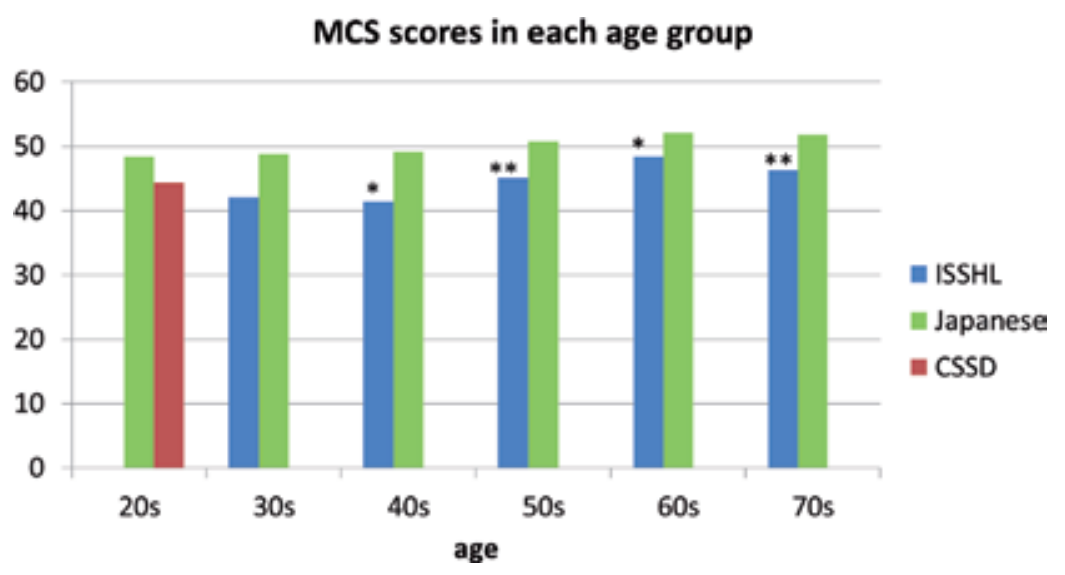

Figure 2.

Mental component summary (MCS) scores of SF-36 in patients with ISSHL and congenital SSD. The graph was created using the data from Sano et al. [8]. "Japanese" indicates age-matched Japanese average scores. The results of 20s group of ISSHL and other than 20s groups of congenital SSD (CSSD) are not indicated because their numbers of patients were too small. P-values are presented as a comparison of scores between each age group of ISSHL or congenital SSD and the age-matched Japanese average: ${ }^{*} p<0.05,{ }^{* *} p<0.01$.

Individuals who had normal hearing in both ears before the onset of ISSHL suddenly lose the ability of spatial hearing, and they instantly begin and remain to feel discomfort in noisy environment. This symptom is important for understanding the QOL problems associated with ISSHL.

\section{Therapeutic interventions for irreversible unilateral sensorineural hearing loss caused by ISSHL}

As mentioned earlier, patients with ISSHL have several hearing-related symptoms, which can be divided into four categories: hearing difficulty, disability of spatial hearing, discomfort, and tinnitus. Hearing difficulty includes several situations, such as conversation with several people and speech perception in noise. The efficacy of therapeutic interventions for patients with ISSHL needs to be evaluated against those four categories. The available methods for evaluating those four categories are summarized in Table 1.

The degree of unilateral hearing loss caused by ISSHL varies from mild to profound, and the selection of interventions depends on the degree of hearing loss. The interventions for ISSHL patients with severe-to-profound hearing loss can be considered the same as those for SSD patients, which include cochlear implant (CI), bone-anchored hearing aids (BAHAs), and contralateral routing of signals (CROS)

\begin{tabular}{lll}
\hline & Objective evaluation & Subjective evaluation \\
\hline Hearing difficulty & Speech discrimination test & SSQ/APHAB/GHABP \\
\hline Spatial hearing & Sound localization & SSQ \\
\hline Discomfort & Not available & Not available \\
\hline Tinnitus & Not available & THI/VAS \\
\hline
\end{tabular}

SSQ, speech, spatial and qualities of hearing scale; $A P H A B$, abbreviated profile of hearing aid benefit; GHABP, Glasgow hearing aid benefit profile; THI, tinnitus handicap inventory; VAS, visual analog scale.

Table 1.

Evaluation methods for four major problems in patients with ISSHL. 
hearing aid. If hearing loss is mild to moderate, a conventional air- conducted hearing aid may be the primary treatment. These treatments are described below.

\subsection{Therapeutic effects for patients with SSD}

The methods for evaluating the therapeutic effects of interventions for patients with SSD have generally included sound localization test, speech comprehension in noise, and subjective evaluation. The effects of the BAHA, CROS hearing aid, and $\mathrm{CI}$ interventions are summarized below.

\subsubsection{BAHA and CROS hearing aid}

The BAHA and CROS hearing aid have essentially the same characteristics in that a microphone is placed on the affected ear side and the sound is heard in the normal ear. Whereas a BAHA transmits sound via bone conduction, a CROS hearing aid transmits sound to a receiver on the normal ear by wireless or wired transmission. The therapeutic effect of these hearing aids can be summarized from the results of two systematic reviews for adult-acquired SSD [10,11].

For sound localization by BAHAs, Kim et al. [10] reported that the percentage of correct sound localization was $13-65.8 \%$ before BAHA implantation and 15-68.5\% after implantation, showing no significant difference in six studies. In a recent study, Agtrberg et al. [12] reported that BAHA neither improved nor deteriorated the localization abilities of patients with SSD. Kitterick et al. [11] reported that sound localization was not changed by CROS hearing aids in five studies and a significant deficit was indicated in one study. Therefore, it can be concluded that there is no improvement in sound localization with BAHAs or CROS hearing aids.

Speech comprehension in noise improves under certain conditions. Kim et al. [10] reported that in the situation of speech coming from the front and noise coming from the normal ear side, speech discrimination was statistically significantly improved after BAHA implantation in four out of six studies. Kitterick et al. [11] conducted a meta-analysis using data from the Hearing in Noise Test. A significant benefit was identified in the situation of speech coming from the front and noise coming from the normal ear side for both BAHAs and CROS hearing aids; however, a significant deficit was identified for both devices in the situation of noise coming from the affected ear side. The effects of BAHAs and CROS hearing aids are generally similar, with the former thought to be slightly superior. For the subjective evaluation of the benefits and adverse effects of the interventions, the abbreviated profile of hearing aid benefit (APHAB) and the Glasgow hearing aid benefit profile (GHABP) have been widely used. Kitterick et al. conducted a meta-analysis using data from the APHAB before and after the use of BAHAs and CROS hearing aids. Significant benefits of the BAHA were found for three subscales, reverberation, ease of communication, and background noise, but not for aversion to loud sound. Significant benefits of the CROS hearing aid were also found for two subscales: background noise and reverberation.

\subsubsection{Cochlear implant}

$\mathrm{CI}$ is a method of treating the deaf ear itself so that it can regain hearing ability. Therefore, the treatment concept is essentially different from the BAHA and CROS hearing aid. A study of CI for adult-acquired SSD was reviewed. Two systematic reviews $[13,14]$ and several subsequent reports [15-18] yielded similar results. These are summarized as follows. 
For sound localization, Kitterick et al. [11] reported that only one of the three studies showed statistically significant improvement after CI surgery. Although most of studies reported improvement of sound localization after CI, a metaanalysis could not be conducted because of heterogeneous methodologies.

For speech comprehension in noise, Blasco and Redleaf [12] conducted a metaanalysis and reported in the situation where both speech and noise were coming from the front; the signal-to-noise ratio for speech perception in noise was significantly improved following CI. However, in the situation where speech was coming from the front and noise was coming from the affected side, no improvement was observed.

For the subjective evaluation, Kitterick et al. [11] conducted a meta-analysis using data from the speech, spatial and qualities of hearing scale (SSQ) [19]. They found significant improvement for all three subscales: speech, spatial, and "other" qualities.

For the subjective evaluation of severity of tinnitus, Blasco and Redleaf [13] conducted a meta-analysis using a visual analog scale from three studies and found statistically significant improvement following CI implantation.

Overall, an important difference in the results of CI from those of the BAHA and CROS hearing aid is that there was a possibility of improvement for sound localization and spatial hearing ability. It is considered that these two factors are associated with each other. In addition, Legaris et al. reported that cortical reorganization and restoration of binaural function in the brain might be produced after 1 year of experience with CI in adult SSD patients by evaluation of cortical auditory evoked potential changes [20].

\subsection{Potential of interventions for ISSHL patients with severe-to-profound hearing loss}

BAHAs and CROS hearing aids improve hearing from the deaf side. Improvement in speech comprehension in noise can be expected when the speech comes from the deaf side or front and the noise comes from the normal ear side. However, the ability for sound localization cannot be expected, and it is not possible to restore spatial hearing function. Therefore, among the associated problems for patients with ISSHL with severe-to-profound hearing loss, both devices seem to give no benefit for spatial hearing, discomfort, or tinnitus.

A CI improves speech comprehension in noise at least as well as the BAHA or CROS hearing aid. A CI also seems to have potential to improve sound localization, which could lead to restoration of spatial hearing ability. The SSQ subjective evaluation contains many assessment items related to spatial hearing [19], and the scores of spatial hearing were reported to improve after cochlear implantation. Although there have been no reports directly evaluating "hearing-related discomfort" as an important symptom of ISSHL, it may be improved if spatial hearing ability can be restored. Direct evaluation of this symptom is needed in future assessments. Patients with tinnitus can also be expected to experience improvement with a CI $[13,15,18]$. Overall, although further investigation is needed, a CI has the potential to improve speech comprehension in noise, spatial hearing, and tinnitus and may also improve discomfort. The indication of CI in patients with ISSHL is the confirmation of cochlear pathogenesis, and relatively early surgery after ISSHL onset should be considered [18].

\subsection{Potential of interventions for ISSHL patients with mild-to-moderate hearing loss}

Conventional hearing aids are indicated for ISSHL patients with unilateral mildto-moderate hearing loss. However, patients with mild-to-moderate hearing loss on 
the affected ear and normal hearing on the opposite ear are less likely to realize the benefits of hearing aids on the affected ear and are often unable to wear them. Since some degree of auditory function remains in the affected ear, symptoms such as difficulty in hearing and impaired sound localization are milder than in patients with SSD, and, as a result, the beneficial effect of wearing a hearing aid seems to be difficult to perceive subjectively and to detect objectively. In addition, Kumpik et al. reviewed from several studies that horizontal localization by adult humans can adapt to varying degree to asymmetric hearing loss induced by occluding one ear [21]. Therefore, the abilities of sound localization and spatial hearing may be spontaneously restored in some degree in the patients with unilateral mild-to-moderate hearing loss. There do not seem to be any previous reports that examined the effect of hearing aids for hearing disability in patients with unilateral mild-to-moderate hearing loss.

Hearing-related discomfort is also common in ISSHL patients with unilateral moderate hearing loss, but the mechanism may differ from that in patients with unilateral severe-to-profound hearing loss. Patients with moderate hearing loss are more likely to have discomfort with sounds heard on the affected side, that is, increased loudness of noise caused by the recruitment phenomenon or distortion caused by the impairment of frequency selectivity function is unpleasant. Therefore, it is unlikely that a hearing aid will improve excessive loudness and distortion of sound.

On the other hand, tinnitus symptoms are more common in patients with mildto-moderate hearing loss due to ISSHL. Tinnitus retraining therapy using a hearing aid as a means of sound therapy has been widely conducted. At present, there is no high-quality evidence from systematic reviews [22], but improvement in the Tinnitus Handicap Inventory or visual analog scales has been widely recognized. In the future, it will be necessary to establish evidence of tinnitus improvement and to evaluate speech comprehension in noise and sound localization as well as hearingrelated discomfort in ISSHL patients with mild-to-moderate hearing loss.

\section{Summary}

ISSHL is an important cause of persistent unilateral sensorineural hearing loss that affects thousands of new patients annually in Japan. The problems caused by ISSHL can be categorized into four factors: hearing difficulty, deterioration of spatial hearing, hearing-related discomfort, and tinnitus. The interventions that have been used to treat patients with unilateral hearing loss can be adapted to patients with ISSHL. The expected benefits of interventions for ISSHL patients are shown in Table 2. Although there are presently no treatments that provide satisfactory outcomes, $\mathrm{CI}$ is possibly the current most effective means of restoring some

\begin{tabular}{llll}
\hline Grade of hearing loss & & Severe-deaf & Mild-moderate \\
\hline Intervention & BAHA/CROS & CI & Conventional HA \\
\hline Speech in noise & Partially improved & Partially improved & $?$ \\
\hline Spatial hearing & $\rightarrow$ & Possibly improved & $?$ \\
\hline Discomfort & $?$ & $?$ & $?$ \\
\hline Tinnitus & $\rightarrow$ & Improved & Improved \\
\hline
\end{tabular}

BAHA, bone-anchored hearing aid; CROS, contralateral routing of signals; CI, cochlear implant; HA, hearing aid; $\rightarrow$, not improved; ?, not available for applicable investigations.

Table 2.

Expected effect of interventions for patients with ISSHL. 
of the lost binaural functions in patients with ISSHL who have severe-to-profound hearing loss. On the contrary, the effect of BAHA and CROS is quite restricted for such patients.

In the future, it will be necessary to unify evaluation methods for sound localization, speech comprehension in noise, and subjective questionnaires. Health-related QOL should be a component of the subjective assessments, and "hearing-related discomfort," which negatively impacts QOL for ISSHL patients, must be included as a subjective evaluation item.

\section{Conflict of interest}

The author has no conflict of interest to declare.

\section{Author details}

Hajime Sano

Department of Rehabilitation, School of Allied Health Sciences, Kitasato University, Japan

*Address all correspondence to: sanohj@med.kitasato-u.ac.jp

IntechOpen

(C) 2019 The Author(s). Licensee IntechOpen. This chapter is distributed under the terms of the Creative Commons Attribution License (http://creativecommons.org/licenses/ by/3.0), which permits unrestricted use, distribution, and reproduction in any medium, provided the original work is properly cited. (cc) BY 


\section{References}

[1] Kuppler K, Lewis M, Abdele KE. A review of unilateral hearing loss and academic performance: Is it time to reassess traditional dogmata? International Journal of Pediatric Otorhinolaryngology. 2013;77:617-612. DOI: 10.1016/1.ijporl.2013.01.014

[2] Schreiber BE, Agrup C, Haskard DO, Luxon LM. Sudden sensorineural hearing loss. Lancet. 2010;375:1203-1211. DOI: 10.1016/ S0140-6736(09)62071-7

[3] Nakashima T, Sato H, Gyo K, Hato N, et al. Idiopathic sensorineural hearing loss in Japan. Acta Oto-Laryngologica. 2014;134:1158-1163. DOI: 10.3109/00016489.2014.919406

[4] Babu R, Sharma R, Bagley JB, Hatef J, Friedman AH, Adamson C. Vestibular schwannomas in the modern era: Epidemiology, treatment trends, and disparities in management. Journal of Neurosurgery. 2013;119:121-130. DOI: 10.3171/2013.1.JNS121370 Epub: 22 February 2013

[5] Hahimoto S. Epidemiology and pathophysiology of vestibular schwannoma-Current concept. Jikotokei. 2016;88:994-999 (Japanese)

[6] Chiossoene-Kerdel JA, Bagley DM, Stoddart RL, et al. An investigation of the audiologic handicap associated with unilateral sudden sensorineural hearing loss. The American Journal of Otology. 2000;21:645-651

[7] Carlsson PI, Hall M, Lind KJ, et al. Quality of life, psychosocial consequences, and audiological rehabilitation after sudden sensorineural hearing loss. International Journal of Audiology. 2011;50:139-144. DOI: 10.3109/14992027.2010.533705

[8] Sano H, Okamoto M, Ohhashi K, Ino T, Iwasaki S, Ogawa K. Self-reported symptoms in patients with idiopathic sudden sensorineural hearing loss. Otology \& Neurotology. 2013;34:1405-1410. DOI: $10.1097 /$ MAO.0b013e3182a03705

[9] Sano H, Okamoto M, Ohhashi K, Iwasaki S, Ogawa K. Quality of life reported by patients with idiopathic sudden sensorineural hearing loss. Otology \& Neurotology. 2012;34:36-40. DOI: 10.1097/MAO.0b013e318278540e

[10] Kim G, Ju HM, Lee SH, Kim HS, Kwon JK, Seo YJ. Efficacy of boneanchored hearing aids in single-sided deafness: A systematic review. Otology \& Neurotology. 2017;38:473-483. DOI: 10.109/MAO.000000000001359

[11] Kitterick PT, Smith SN, Lucas L. Hearing instruments for unilateral severe-to-profound sensorineural hearing loss in adults: A systematic review and meta-analysis. Ear and Hearing. 2016;37:495-507. DOI: 10.1097/ AUD.0000000000000313

[12] Agtrberg MJH, Snik AFM, Van de Goor RMG, Hol MKS, Van Opstal AJ. Sound localization performance of patients with single-side deafness is not improved when listening with a boneconduction device. Hearing Research. 2019;372:62-68. DOI: 10.1016/j. heares.2018.04.007. Epub: 19 April 2018

[13] Blasco MA, Redleaf MI. Cochlear implantation in unilateral sudden deafness improves tinnitus and speech comprehension: Meta-analysis and systematic review. Otology \& Neurotology. 2014;35:1426-1432. DOI: 10.1097/MAO.0000000000000431

[14] Grossmann W, Brill S, Moeltner A, Mlynski R, Hagen R, Radeloff A. Cochlear implantation improves spatial release from masking and restores localization abilities in single-sided deaf patients. Otology \& Neurotology. 
2016;37:658-664. DOI: $10.1097 /$

MAO.0000000000001043

[15] Louza J, Hempel JM, Krause E, Berghaus A, Muller J, Braun T.

Patient benefit from cochlear implantation in single-sided deafness:

A 1-year follow-up. European Archives of Oto-Rhino-Laryngology. 2017;27:2405-2409. DOI: 10.1097/ MAO.0000000000000841

[16] Buss E, Dillon MT, Rooth MA, King ER, Deres EJ, Buchman CA, et al. Effects of cochlear implantation on binaural hearing in adults with unilateral hearing loss. Trends in Hearing. 2018;22:2331216518771173. DOI: $10.1177 / 2331216518771173$

[17] Prejban DA, Hamzavi JS, Arnoldner C, Liepins R, Honeder C, Keider A, et al. Single sided deaf cochlear implant users in the difficult listening situation: Speech perception and subjective benefit. Otology \& Neurotology. 2018;39:e803-e809. DOI: 10.1097/ MAO.0000000000001963

[18] Lin YW, Cheng X, Chen B, Peng $\mathrm{K}$, Ishiyama $\mathrm{A}, \mathrm{Fu} \mathrm{QJ}$. Effect of tinnitus and duration of deafness on sound localization and speech recognition in noise in patients with single-side deafness. Trends in Hearing. 2018;22:2331216518813802. DOI: $10.1177 / 2331216518813802$

[19] Gatehouse S, Noble W. The speech, spatial and qualities of hearing scale (SSQ). International Journal of Audiology. 2004;43:85-99

[20] Legaris E, Galvin J, Roux S, Gomot M, Aoustin JM, Marx M. Cortical reorganization after cochlear implantation for adults with single-sided deafness. PLoS ONE; 2018;13:e0204402. DOI: 10.1371/ journal.pone.0204402

[21] Kupik DP, King AJ. A review of the effects of unilateral hearing loss on spatial hearing. Hearing Research. 2019;372:17-28. DOI: 10.1016/j. heres.2018.08.003

[22] Sereda M, Xia J, EI Refaie A, Hall DA, Hoare DJ. Sound therapy (using amplification devices and/ or sound generators) for tinnitus. Cochrane Database of Systematic Reviews. 2018;27(12):CD013094. DOI: 10.1002/14651858.CD013094.pub2 



\title{
The Site of Lesion in Hearing Loss: Advances in Otoneuroradiology
}

\author{
Giorgio Conte, Silvia Casale, Sara Sbaraini, \\ Federica Di Berardino and Diego Zanetti
}

\begin{abstract}
The last decade has witnessed significant advances in imaging of the middle and inner ear and the auditory pathways. High resolution computerized tomography (CT) scanners and new magnetic resonance (MR) sequences have been implemented in clinical practice as valuable supportive tools for the Audiologist in the identification of the site of lesion and for the surgical planning by the Otologist. The purpose of this chapter is to review the current advanced methods of neuroradiological evaluation of patients with sensorineural hearing loss (SNHL), either congenital or acquired, especially focusing on the assessment of candidates to cochlear implantation (CI), with plenty of explicative images.
\end{abstract}

Keywords: sensorineural hearing loss, congenital inner ear malformations, acquired inner ear disorders, flat panel CT, high-resolution MR-sequences

\section{Introduction}

The imaging assessment of the inner ear and the auditory pathway requires high-resolution techniques because their anatomical structures are small and complex. In the last 10 years, computed tomography (CT) and magnetic resonance (MR) has reached much higher spatial resolution for bony and neural structures.

Multi-section CT (MSCT) is the technique of choice for the study of the temporal bone, thanks to its high spatial resolution. However flat panel CT (FPCT) constitutes the newest alternative technique, since it guarantees some additional advantage compared with MSCT in terms of ultra-high isotropic spatial resolution $\left(\sim 150 \times 150 \times 150 \mu \mathrm{m}^{3}\right)$ and reduction of the effective dose of up to $40 \%$ [1-3].

On the other hand, 3 Tesla MR scanners, thanks to the high-resolution sequences such as tridimensional (3D) T2 weighted sequence (3D-T2 $\mathrm{w}$, spatial resolution: $0.4 \times 0.4 \times 0.6 \mathrm{~mm}^{3}$, field of view: $140 \times 140 \mathrm{~mm}$, time of scan: $5^{\prime} 32^{\prime \prime}$ ) and 3D Fluid Attenuated Inversion Recovery (FLAIR), have provided new insight to detect changes in the inner ear $[4,5]$. MR is technique of choice to study the peripheral and central auditory pathways.

The purposes of this chapter is to review the state of the art of pre-operative neuroradiological assessments of patients with sensorineural hearing loss (SNHL) especially focusing on candidates to a cochlear implant (CI). Advanced imaging of the morphology and the integrity of anatomical structures will be presented in order to show the current capabilities of correct site of lesion identification. 


\section{Inner ear and cochlear-vestibular nerve}

\subsection{Congenital disorders}

Inner ear can be affected by malformative and acquired anomalies, which can cause profound to severe hearing impairment. FPCT scan is more accurate than conventional MSCT in the morphologic evaluation of inner ear; FPCT allows an excellent examination of the $32-\mathrm{mm}$ spiral canal of the snail-shaped cochlea, that winds 2 and $1 / 2$ turns around the modiolus. These turns are separated by interscalar septa, where defects of it and of the modiolus can describe different types of incomplete partition of the cochlea. Sennaroğlu and Bajin summarized the findings of abnormalities involving each inner ear structure, as described in Table 1 [6].

The most common cochlea anomalies are incomplete partition type I (IP-I) and incomplete partition type II (IP-II). In the IP-I, the cochlea results in a cystic appearance, due to the lack of the entire modiolus and the cribriform area, associated with a large cystic vestibule. In the IP-II (classic Mondini deformity), the cochlea forms a cystic apex, due to the coalescence of the middle and the apical turns, accompanied by a dilated vestibule and enlarged vestibular aqueduct

(Figure 1). A rare disorder associated with congenital mixed hearing loss is incomplete partition type III, an X-Linked inner ear anomaly, caused by the absence of the bony modiolus and of the septum between the base of the cochlea and the internal auditory canal, with no associated anomalies in the vestibular structures of the inner ear (Figure 2) [6, 7]. This anomaly is associated with fixed stapes footplate, which represents a surgical risk of perilymph gusher during stapedectomy, and it represents even a risk of misdirected insertion of the cochlear electrode through the internal acoustic canal, inside the cerebellopontine angle against the brain stem, during cochlear implantation (Figure 3).

Cochlear malformations can be accompanied by anomalies of the vestibule or of the semicircular canals, resulting in aplasia, hypoplasia or dilatation [8].

Another cause of SNHL is the presence of enlarged vestibular aqueduct described as larger than $1.5 \mathrm{~mm}$ at the midpoint in the axial plane between the common crus and the external aperture, associated with a normal cochlea, vestibule and semicircular canals [8]. The Pöschl projection improves the accuracy in the measurement of the aqueduct. It is defined as $45^{\circ}$ from either the sagittal or coronal

\begin{tabular}{ll}
\hline Type of IEM & Radiological findings \\
\hline Complete labyrinthine aplasia & Absent labyrinth \\
\hline Rudimentary otocyst & Incomplete millimetric otic capsule remnant \\
\hline Cochlear aplasia & Absent cochlea \\
\hline Common cavity & Round or ovoid cystic structure for cochlea and vestibule \\
\hline Cochlear hypoplasia & Cochlear size small \\
\hline Incomplete partition—I & Cystic cochlea \\
\hline Incomplete partition-II & Cystic cochlea apex \\
\hline Incomplete partition-III & Modiolus absent, interscalar septa present \\
\hline Enlarged vestibular aqueduct & Normal cochlea with enlarged vestibular aqueduct \\
\hline Cochlear acqueduct abnormalities & Narrow or absent cochlear acqueduct \\
\hline
\end{tabular}

Table 1.

Sennaroğlu and Bajin's description of the findings of abnormalities involving inner ear structures. 

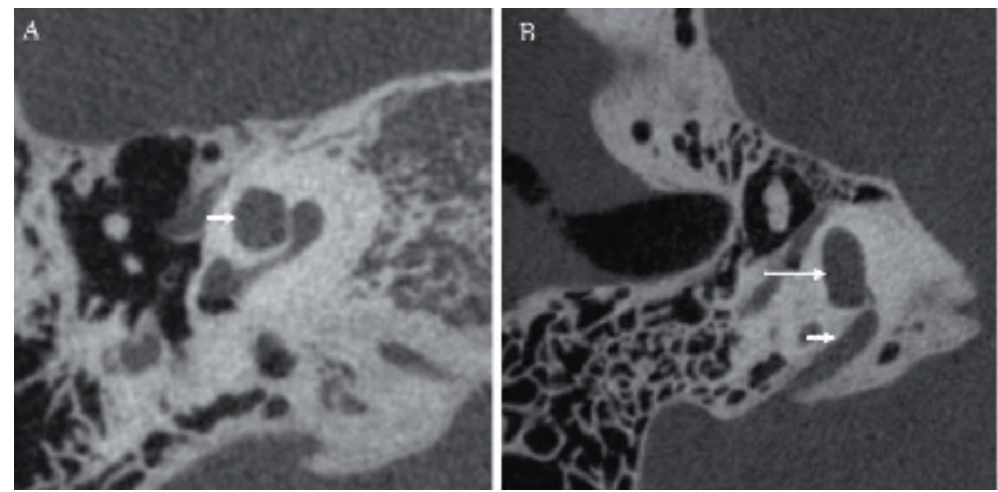

Figure 1.

FPCT on axial plane showing IP-II deformity (classic Mondini deformity): the cochlea forms a cystic apex, due to the fusion of the middle and the apical turns (arrow, A), accompanied by a dilated vestibule (long arrow, B) and enlarged vestibular aqueduct (short arrow, B).

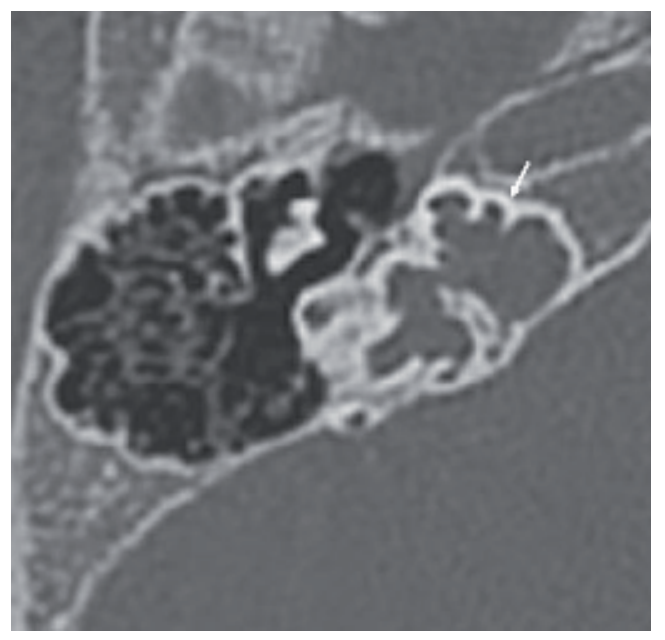

Figure 2.

FPCT on axial plane showing IP-III deformity (arrow), caused by the absence of the bony modiolus and of the septum between the base of the cochlea and the internal auditory canal.

planes or perpendicular to the long axis of the pyramid, or parallel to the superior semicircular canal (Figure 4) [9].

Enlarged vestibular aqueduct, accompanied by enlarged endolymphatic sac, can be associated with other cochlear and vestibular anomalies, which can affect therapeutic strategies: scalar asymmetry with enlargement of anterior chamber composed of the scala vestibuli and the scala media, or modiolar deficiency that shows a flattened and attenuated modiolus, well evaluated in 3D-T2w (Figure 5).

It is important to identify the atresia or the hypoplasia of the cochlear canal, by measuring the length and the width of the bony canal of the cochlear nerve, respectively obtained by drawing a perpendicular line from the base of the modiolus to the inner margin of the fundus of the internal auditory canal (IAC) and by drawing a line along the inner bony margins (Figure 6). The measurements can detect a hypoplastic bony canal, which may be indicative of a embryologic malformation of the cochlear-vestibular nerve (CVN), in patients with congenital SNHL [10-12].

The presence of aplasia or hypoplasia of the CVN must be ruled out since may affect the choice of surgical technique and $\mathrm{CI}$ device, as well as the performance 


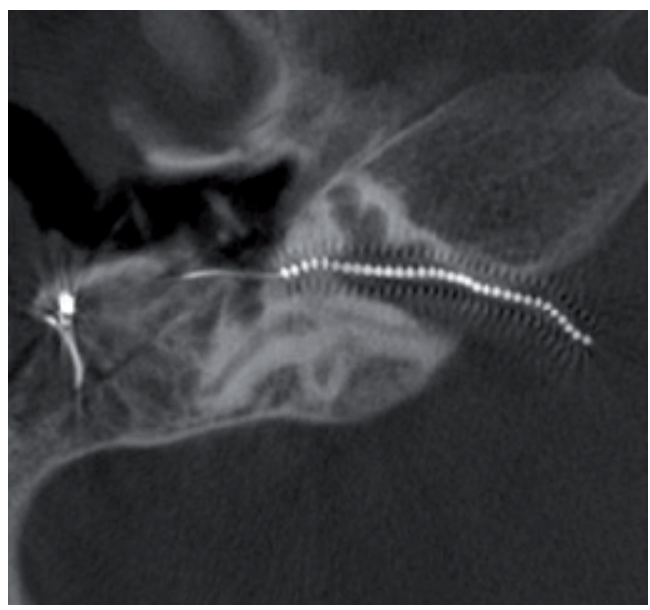

Figure 3.

FPCT on axial plane showing a complicated insertion of cochlear implant in IP-III deformity. The CI electrode enters the internal acoustic canal, reaching the cerebellopontine angle.
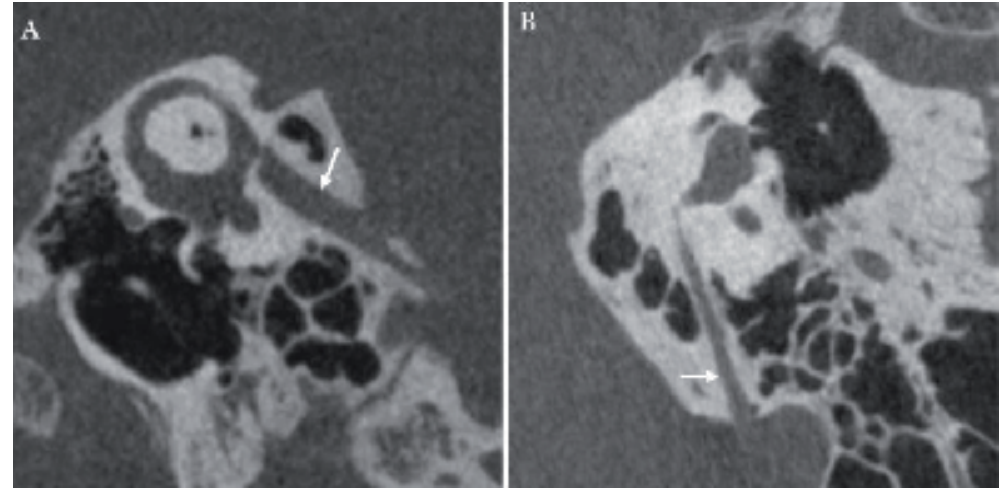

Figure 4 .

FPCT on Pöschl projection (A), which is reconstructed approximately $45^{\circ}$ from either the sagittal and coronal planes, parallel to the loop of the superior semicircular canal, and axial plane (B) between the common crus and the external opening, showing an enlarged vestibular aqueduct (arrows).

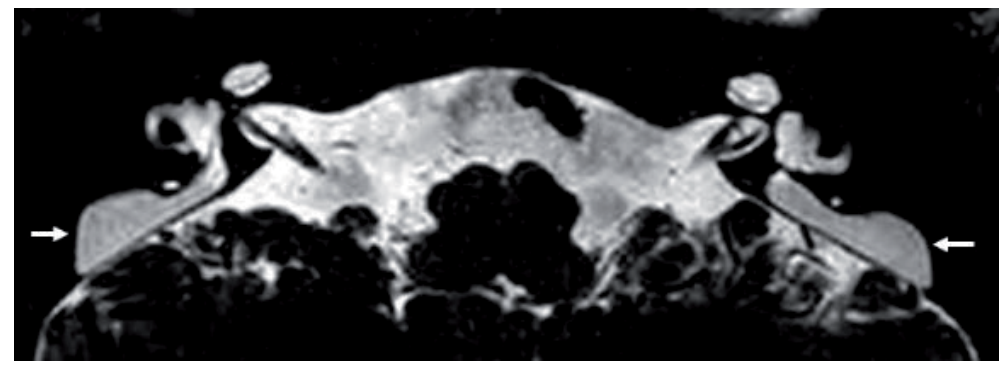

Figure 5.

3D-T2w on 3 Tesla MR on axial plane showing enlarged endolymphatic sacs (arrows), associated with enlarged vestibular aqueducts.

outcome following CI; in patients whose cochlear nerves are missing, an auditory brain stem implant should be considered [13].

The presence of a normal cochlea does not exclude the agenesis of the cochlear nerve because of the different embryogenesis of the otic labyrinth and its neural 
elements. Nevertheless the cochlear nerve malformations are often associated with labyrinthine abnormalities [10]. As mentioned above, the hypoplasia of the cochlear canal seen on CT scan is a sensitive indicator of CVN malformation: in that case the CT evaluation should be always followed by MR examination since the $3 \mathrm{D}-\mathrm{T} 2 \mathrm{w}$ is the most valuable tool to assess nerves anomalies.

The relationship between the facial and the CVN within the IAC may vary among individuals (Figures 7 and 8).

\subsection{Acquired disorders}

Among acquired lesions in SNHL, the presence and the extension of a labyrinthine ossification represents another great challenge for the surgeon, owing to difficult insertion of the electrode array. It may occur like "end-stage" of different labyrinthine pathologies, categorized as infective or non-infective, including meningitis, otitis media, trauma, otosclerosis and labyrinthectomy. Fibrosis can precede ossification but extensive fibrosis without ossification rarely occurs.
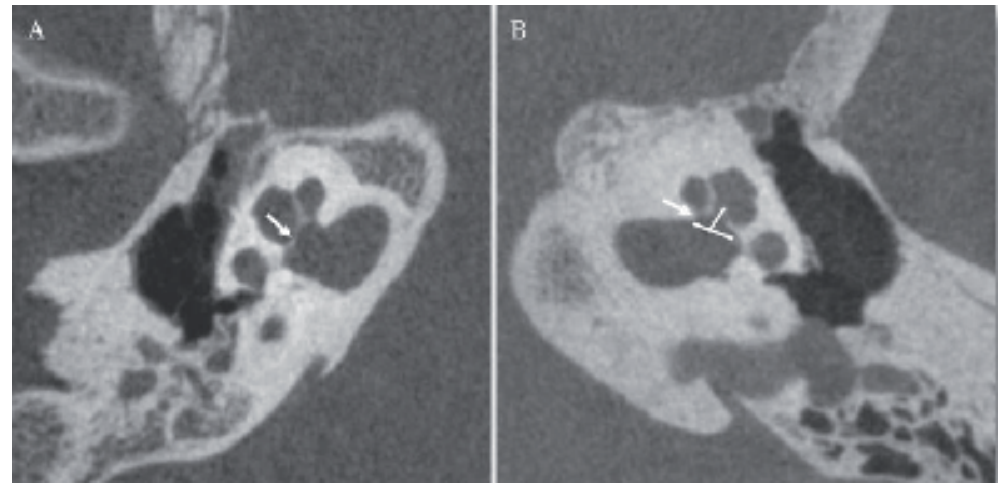

Figure 6.

FPCT on axial plane showing aplasia of cochlear canal (arrow, A) and normal cochlear canal (arrow, B). The measurement of the length and the width of the bony cochlear canal are made by drawing a perpendicular line from the base of the modiolus to the inner margin of the fundus of the internal acoustic meatus and by drawing a line along the inner margins of its bone edges $(B)$.
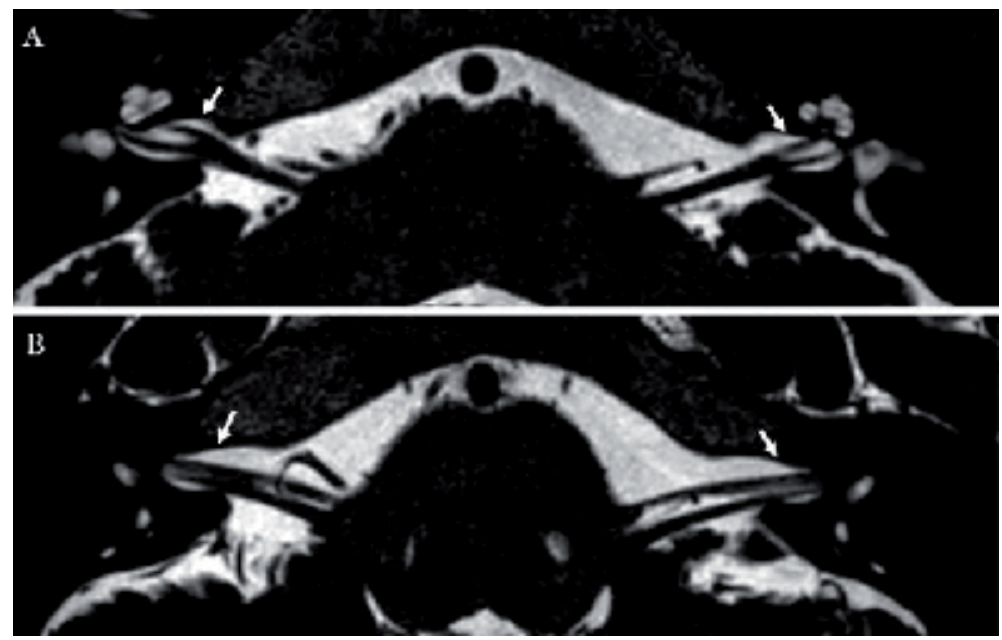

Figure 7.

$3 D-T 2 w$ on 3 Tesla MR on axial plane showing normal appearance of cochlear and inferior vestibular nerves (arrows, A), facial and superior vestibular nerves (arrow, B). 
The stage of fibrosis begins $\sim 2$ weeks after the onset of infection, but CT images remains elusive. Instead, 3D-T2w demonstrates replacement of the normally fluid-filled spaces of the labyrinth, resulting in reduced $\mathrm{T} 2 \mathrm{w}$ signal and shows the presence of labyrinthine enhancement after gadolinium. MR images have the advantage of not only diagnosing cochlear obstruction, but even better sensitivity for estimating the extent of fibrous obstruction, subtle at CT images, thus allowing earlier diagnosis (Figures 9 and 10) [14].

The end stage of the ossification results in the absence of $\mathrm{T} 2 \mathrm{w}$ signal of the labyrinth; this stage is also well seen from CT images (Figure 11).

Reduced T2w signal is reported in vestibular schwannoma, up to $10-20 \%$ of the causes of SNHL, but it always results in intralabyrinthine contrast enhancement (Figure 12).

The management of far advanced otosclerosis may represent another important objective in the era of CI [15]. In the original otosclerosis, the aberrant bone deposition around the stapes footplate results in the impairment of the mechanical transmission of sound, leading to conductive hearing loss. In advanced otosclerosis,
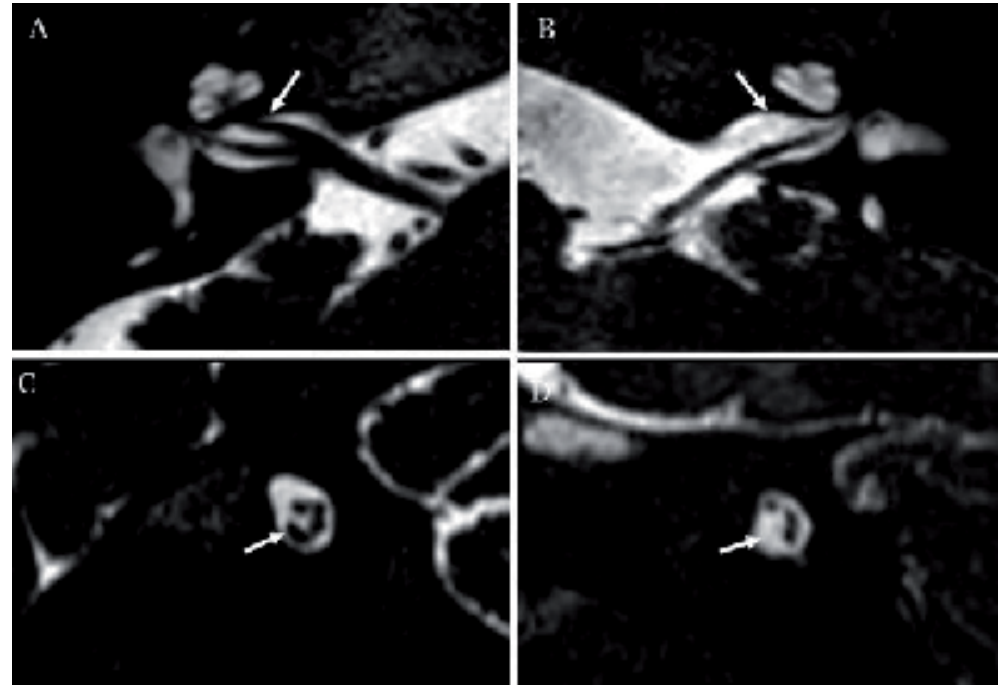

Figure 8.

${ }_{3 D} D-T 2 w$ on 3 Tesla MR showing normal appearance of cochlear nerve on axial (arrow, $A$ ) and sagittal plane (arrow, C) and aplasia of cochlear nerve on axial plane (arrow, B) and on sagittal plane (arrow, D).
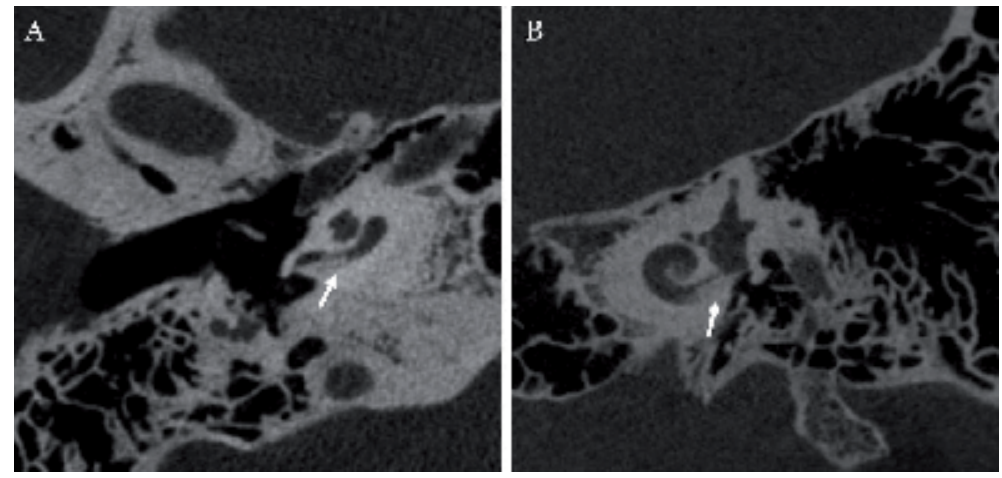

Figure 9.

FPCT on axial $(A)$ and para-coronal $(B)$ planes showing minimal ossification of the basal turn of the cochlea. 

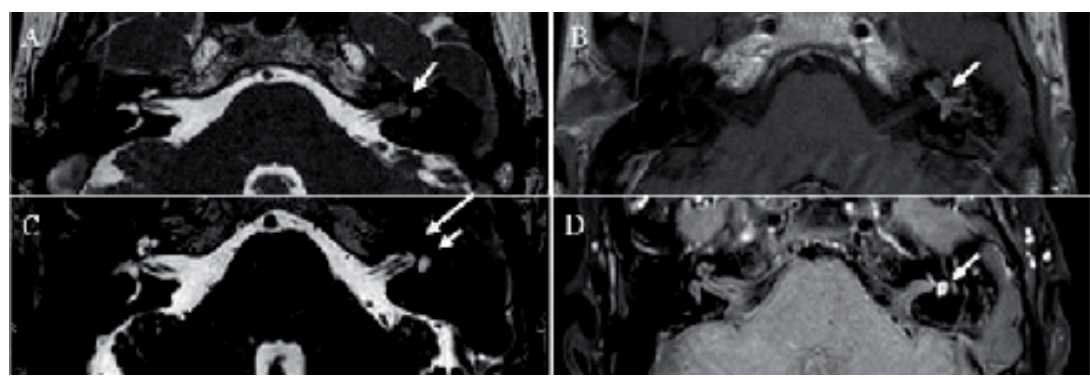

Figure 10.

3-Tesla MR shows loss of signal of the left cochlea and vestibule on axial T2w sequence (arrow, $A$ ), and enhancement after contrast-medium administration on axial T1w (arrow, B), suggesting intralabyrinthine fibrosis. At 1-year follow-up, MRI shows loss of signal of the left cochlea on T2w sequence (long arrow, C), without enhancement after contrast-medium administration, compatible with complete ossification; MR findings suggest that active fibrosis is still present within the left vestibule (short arrow, C and D).
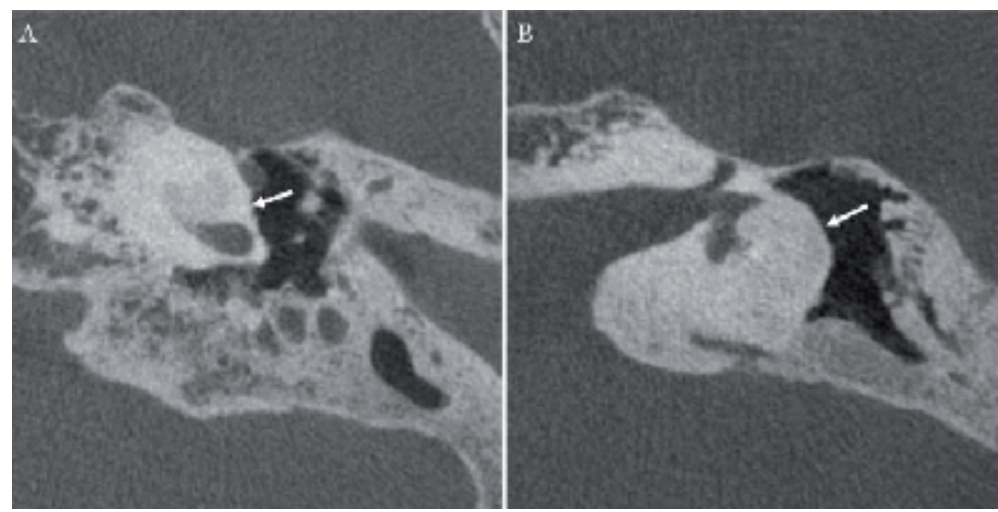

Figure 11.

FPCT on axial plane showing the "end stage" of the ossification of the cochlea (arrow, A) and semicircular lateral canal (arrow, B).

bone deposits can extend into the bony labyrinth of the inner ear, surrounding the cochlea and resulting in a mixed conductive and SNHL. Advanced otosclerosis with cochlear involvement may influence the insertion and the performance of a CI. Despite in these patients the CI has proven to be the most effective treatment modality, endoluminal otospongiotic obstruction may complicate the insertion of the array and alter the spread of the electrical stimulation. Pre-operative CT may clearly detect ossification of the cochlea (Figure 13) [16].

Until recently, MR imaging with and without contrast media has been used only to exclude cochlear causes of sudden SNHL (SSHL), such as a vestibular schwannoma, and rare causes of retrocochlear SSHL including demyelinating disease, brain stem infarctions and cerebellopontine tumors.

Nowadays, pre-contrast T1-weighted and 3D-FLAIR sequence may suggest the pathogenesis of SSHL.

Two specific patterns can be identified: the "vascular" pattern, characterized by the presence of methemoglobin in the inner ear that appears as hyperintense on both pre-contrast T1-weighted and 3D-FLAIR images (Figure 14), and the "inflammatory" pattern which shows high signal only on 3D-FLAIR sequence, due to the presence of proteins in the inner ear fluids (Figure 15). Both patterns can be associated with enhancement on post-contrast 3D-FLAIR which suggests the bloodlabyrinth breakdown [17]. 

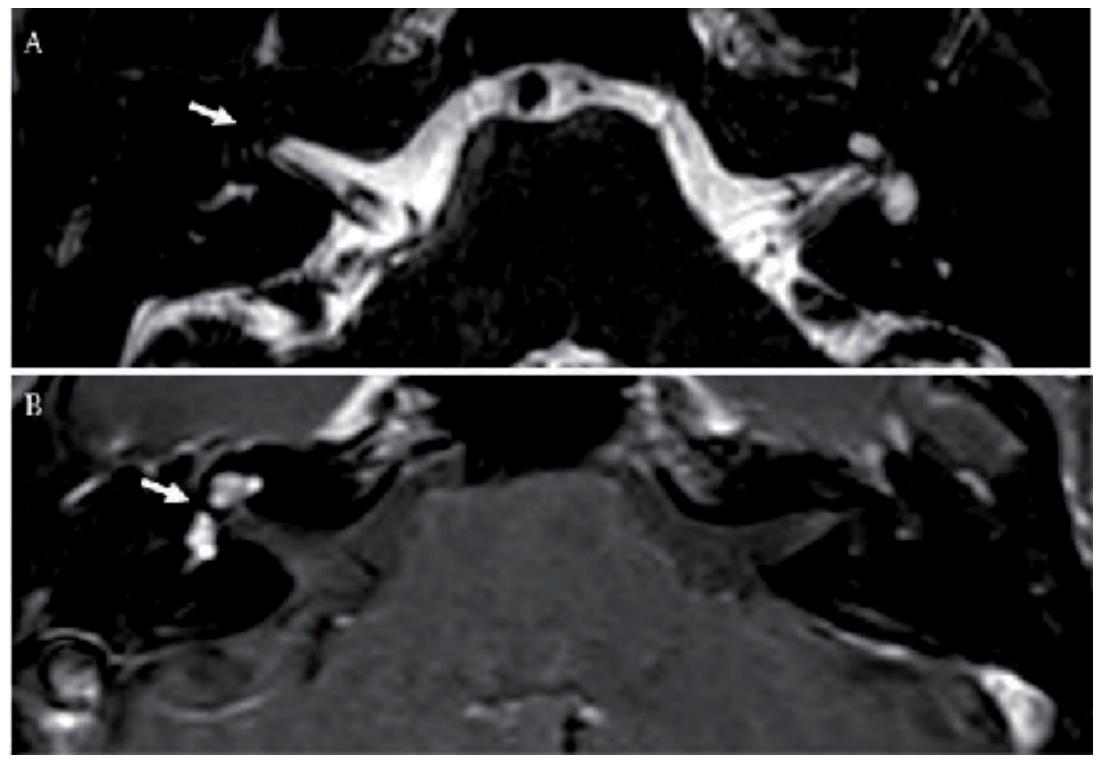

Figure 12.

$M R$ images $3 D-T_{2}(A)$ showing reduced $T_{2}$ signal of the right cochlea and vestibule (arrow); after intravenous contrast-medium administration T1w sequence (B) shows cochlear and vestibular enhancement (arrow), due to intralabyrinthine schwannoma.

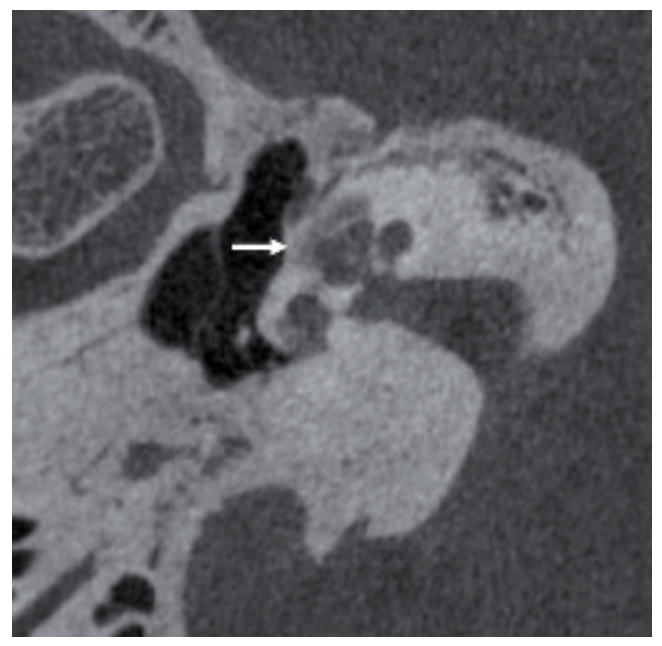

Figure 13.

FPCT on axial plane shows a peri-cochlear hypodensity (arrow) compatible with advanced otosclerosis.

MR sequences, in particular 3D-T2, are excellent to analyze inner ear fluids intensity, and to investigate the morphology of the inner ear structures, cranial nerve VIII, internal auditory canal and cerebellopontine angle $[18,19]$.

Post-contrast 3D-FLAIR sequences are able to identify pathological conditions characterized by blood-labyrinth barrier breakdown, including viral infection, immune-mediated inner ear disease, perilymphatic fistulas $[20,21]$.

Furthermore, the $4 \mathrm{~h}$-delayed 3D-FLAIR sequences on 3 Tesla scanner after intravenous administration of gadolinium-based contrast agent offer enough spatial resolution to detect small endolymphatic structures, such as saccule and utricle; in this sequences the endolabyrinth appears to have a lower signal compared with the surrounding perilymph. 
The Site of Lesion in Hearing Loss: Advances in Otoneuroradiology

DOI: http://dx.doi.org/10.5772/intechopen.90650
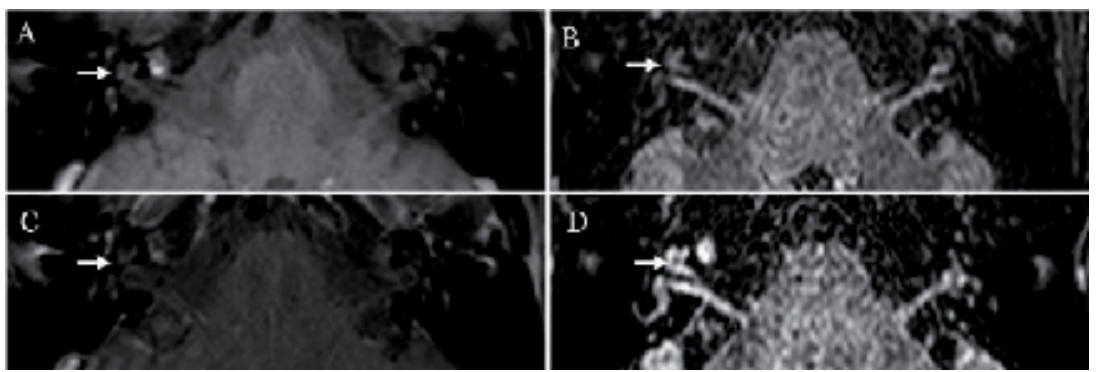

Figure 14 .

Vascular pattern of SSHL. Pre-contrast T1w (A) and $3 D$-FLAIR (B) sequences show a high signal in the right cochlea (arrow), without enhancement on postcontrast $T 1 w$ (arrow, $C$ ). The inner ear enhancement on $3 D$-FLAIR sequence is consistent with blood-labyrinth barrier breakdown of the inner ear (arrow, D).

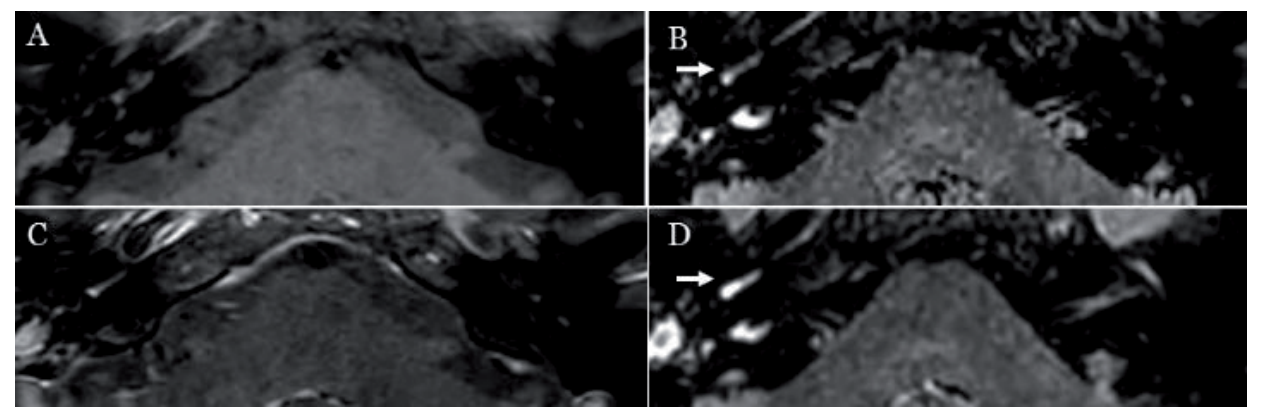

Figure 15.

Inflammatory pattern of SSHL. The pre-contrast T1w (arrow, A) shows no signal abnormalities. The precontrast $3 D$-FLAIR (arrow, B) shows a high signal in the basal turn of the right cochlea. A postcontrast T1-w sequence (arrow, $C$ ) does not show enhancement, whereas a postcontrast $3 D$-FLAIR sequence (arrow, $D$ ) shows the basal turn of the cochlea markedly enhanced on the right side.

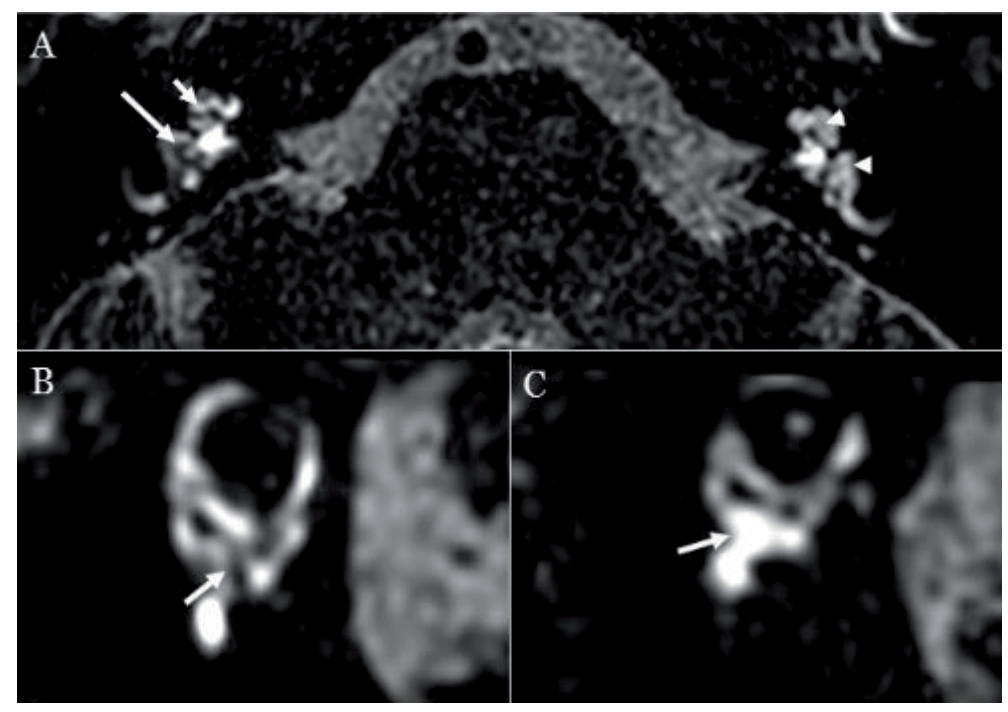

Figure 16.

Axial $3 D$-FLAIR sequence after 4- $h$ from intravenous contrast-medium administration $(A)$ shows right vestibular (long arrow) and cochlear (short arrow) hydrops, compared with the normal left inner ear (arrowheads). On oblique sagittal plane (B), the 4-h delayed postcontrast $3 D$-FLAIR sequence confirms the right vestibular endolymphatic hydrops, in particular of the saccule (arrow), while in the left ear $(C)$, the inferior third of the vestibule is normally filled by perilymph (arrow). 
In patients with Meniere's disease, the pathological finding is represented by endolymphatic hydrops, characterized by a distension of the endolymphatic space of the inner ear into areas that are normally filled with perilymphatic liquid (Figure 16). CI is an effective means of treatment of patients with end-stage Meniere's disease affected by severe-to-profound SNHL [22-24].

\section{Auditory central nervous system}

The auditory central system includes: the cochlear nucleus and the superior olivary complex in the pons, the inferior colliculus in the midbrain, the medial geniculate nucleus of the thalamus and the auditory cortex (i.e. Heschl's gyrus) in the temporal lobe.

Nowadays, MR imaging is the modality of choice to investigate the auditory central pathway, because of its better sensitivity and specificity compared with others neuro-imaging modalities (CT and, in the newborn, ultrasound scanning).

The auditory pathway myelination begins during the fetal life and increases up to the first year of age, reflecting the improvement of the auditory system function. The changes in myelin can be seen using MR imaging by the 37th fetal week in the brainstem and later in the structure higher in the auditory pathway, as early as 10 weeks of age in the medial geniculate nucleus and by 24 weeks in subcortical white matter (Figure 17) [25, 26].

The 3T MR scanner, thanks to its high contrast and spatial resolution, guarantees great sensitivity in the detection of white matter injury, playing an important role in the early diagnosis of some pathologies related with congenital SNHL [27].

In addition to conventional MR sequences, some advanced techniques such as diffusion tensor imaging (DTI) and functional MR imaging (fMRI), may be helpful to provide new insight into structural and functional white matter changes in early stage of congenital SNHL [28]. On the other hand, the investigation of the first step of the central auditory pathway, i.e. cochlear nucleus, is challenging and currently limited in clinical practice. The most promising MR sequences are T2-weighted

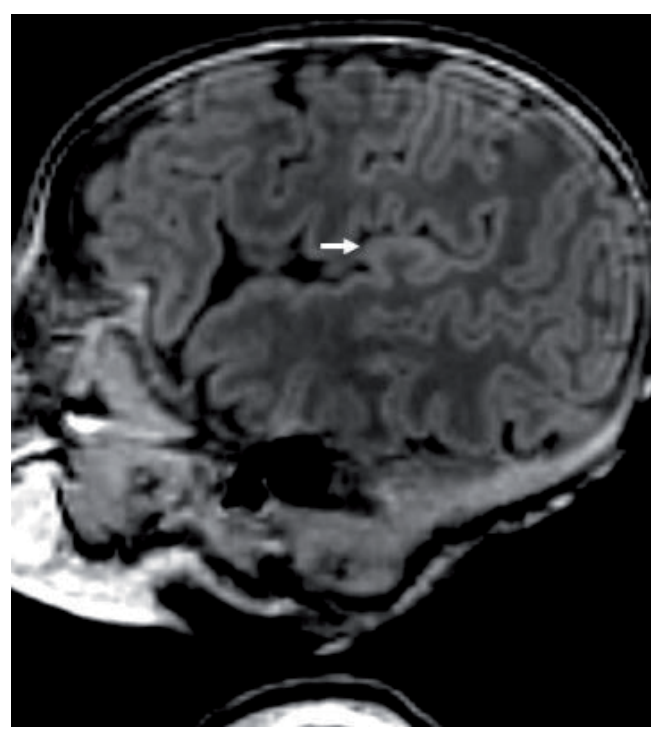

Figure 17.

Sagittal T1w sequence in a healthy 42-weeks newborn shows the normal myelination pattern (as hyper-intense signal) in the cortical rim of the Heschl's girus (arrow). 
gradient-echo (T2GE) imaging and susceptibility weighted imaging (SWI), which ensure a good contrast resolution between hypointensity of the nuclei, due to paramagnetic deposition, and hyperintensity of the surrounding myelinated tissue.

Both environmental and genetic pathologies may lead to congenital SNHL, with possible involvement of any component of acoustic pathway [29, 30].

Frequently, the anatomical structure primarily involved in inherited hearing loss are the inner ear and the CNV (discussed in the previous chapter), while the auditory central pathway is spared by any lesion at the conventional MR investigation.

Conversely, the central system is commonly involved in acquired SNHL disorders. Cytomegalovirus (CMV) is the most common congenital non-genetic cause of SNHL [31]. About $10 \%$ of CMV infection results in several brain abnormalities. At present, an official guideline on the preferred neuroimaging modalities to identify brain abnormalities in patients with CMV infection is lacking; the current practice varies according to center-based protocols [32].

MR imaging is more sensitive than ultrasound scanning in the detection of mild and severe brain abnormalities, such as: microcephaly, cerebellar hypoplasia, cortical anomalies, calcification, white matter changes, polar temporal lesion,
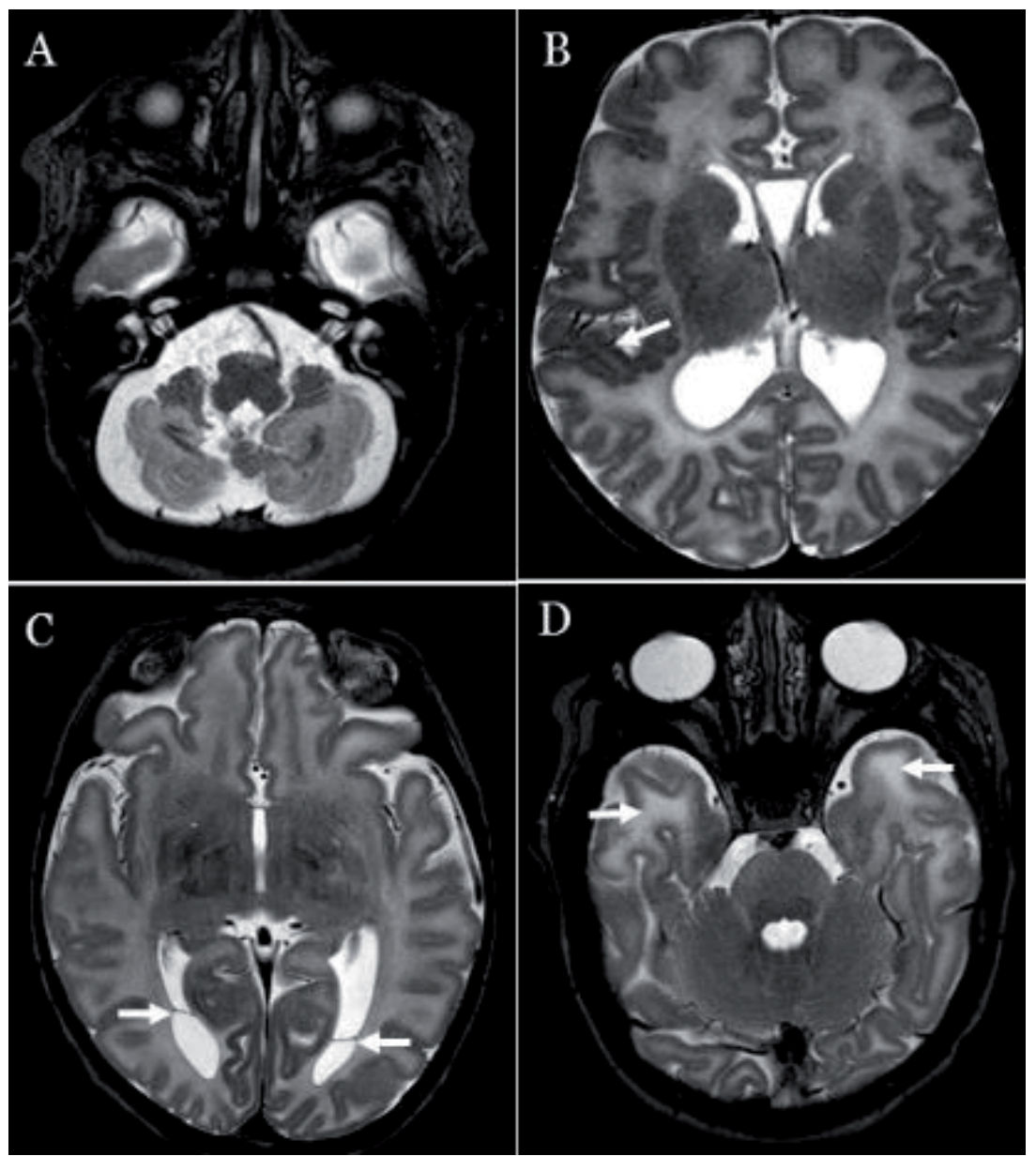

Figure 18.

MR findings in four patients with congenital cytomegalovirus infection: cerebellar hypoplasia $(A)$; perisylvian polymicrogyria $(B)$ that involves the right Heschl's gyrus (arrow); intraventricular septation $(C)$ in the occipital horn of the lateral ventricles (arrows, C); hyperintensity of the anterior temporal white matter (D, arrows). 
sub-ependimal cyst, ventriculomegaly and intraventricular septa (Figure 18). The combination of these malformations depends on gestation age at time of infection $[33,34]$.

Unfortunately, up to date no specific MR finding is significantly associated with the hearing impairment and outcome of treatment [35]. Moreover, all the above mentioned findings are common to several conditions associated with SNHL and should be considered in the differential diagnosis.

Considering others congenital infections, toxoplasmosis and rubella are commonly described as sharing similar pictures with CMV. In countries where the rubella immunization programs are in place, a congenital infection is currently rare; nevertheless, this diagnostic hypothesis should be considered in presence of brain calcifications, white matter lesions, intraventricular septations, subependymal germinolytic cysts and ventriculomegaly. Noteworthy, newborns affected by CMV showing with brain abnormalities are usually asymptomatic from a neurological standpoint, but may develop progressive SNHL only later on in life [36, 37].

Other rare causes of acquired hearing loss, occurring whenever the auditory central pathway is involved in the injury, are focal brain lesions following perinatal ischemic or hemorrhagic event may (Figure 19), hypoxic-ischemic encephalopathy (HIE) (Figure 20) [38, 39]. Therapeutic hypothermia, which is the only approved treatment for HIE, because it reduces the extension of brain lesions, has been unfortunately associated with SNHL. The correlation between imaging and functional outcome is still unknown; up to $10 \%$ of the treated babies develop hearing impairment.

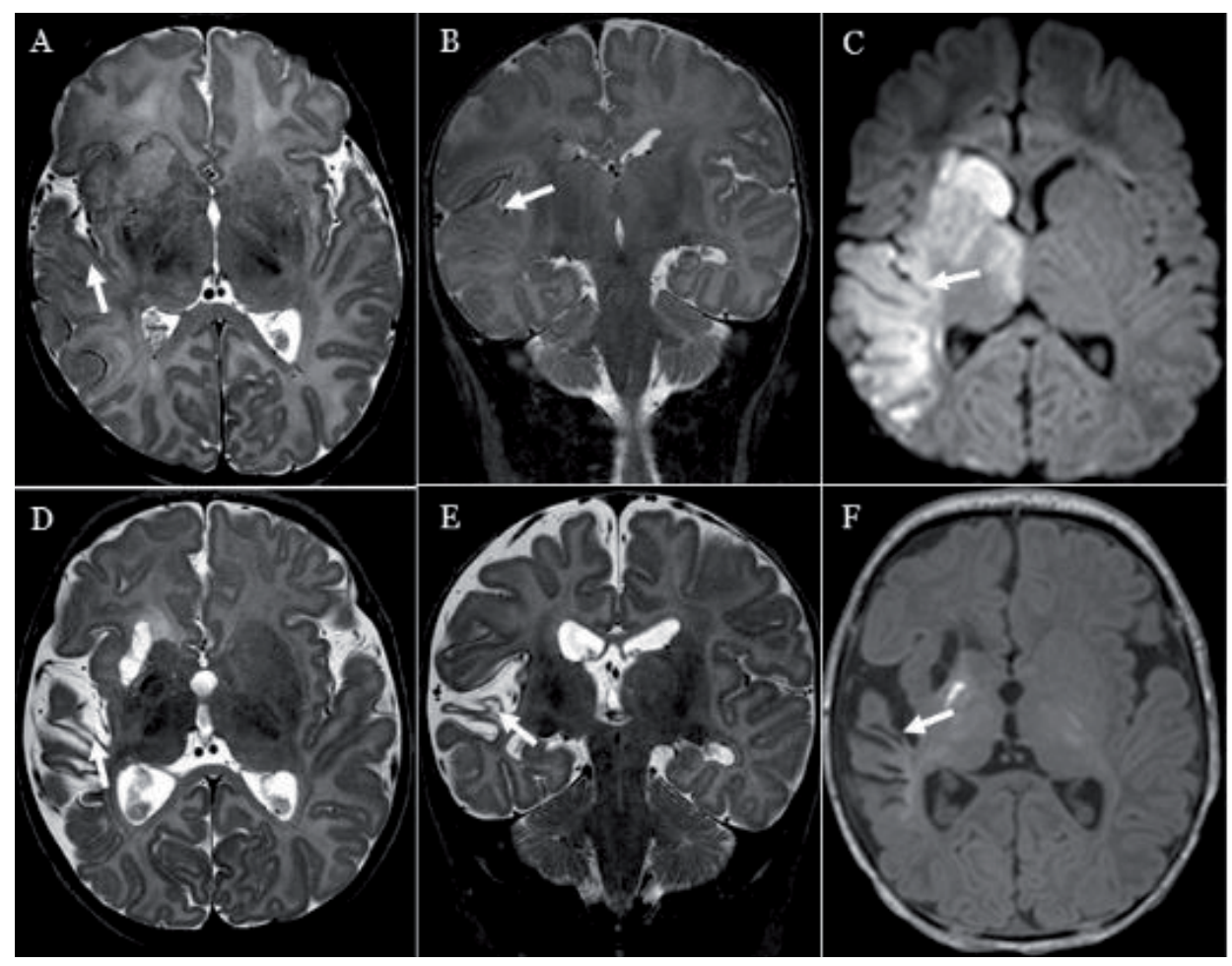

Figure 19.

Images showing the evolution of perinatal ischemic injury involving the right temporal lobe and the right basal ganglia. Axial T2w (A), coronal T2w (B) and DWI (C) images of 2 days newborn during the acute phase of the ischemic injury. Axial T2w (D), coronal T2w (E) and axial T1w (F) images showing the massive damage after 42 days; the involvement of the primary auditory cortex (arrows) caused SNHL. 

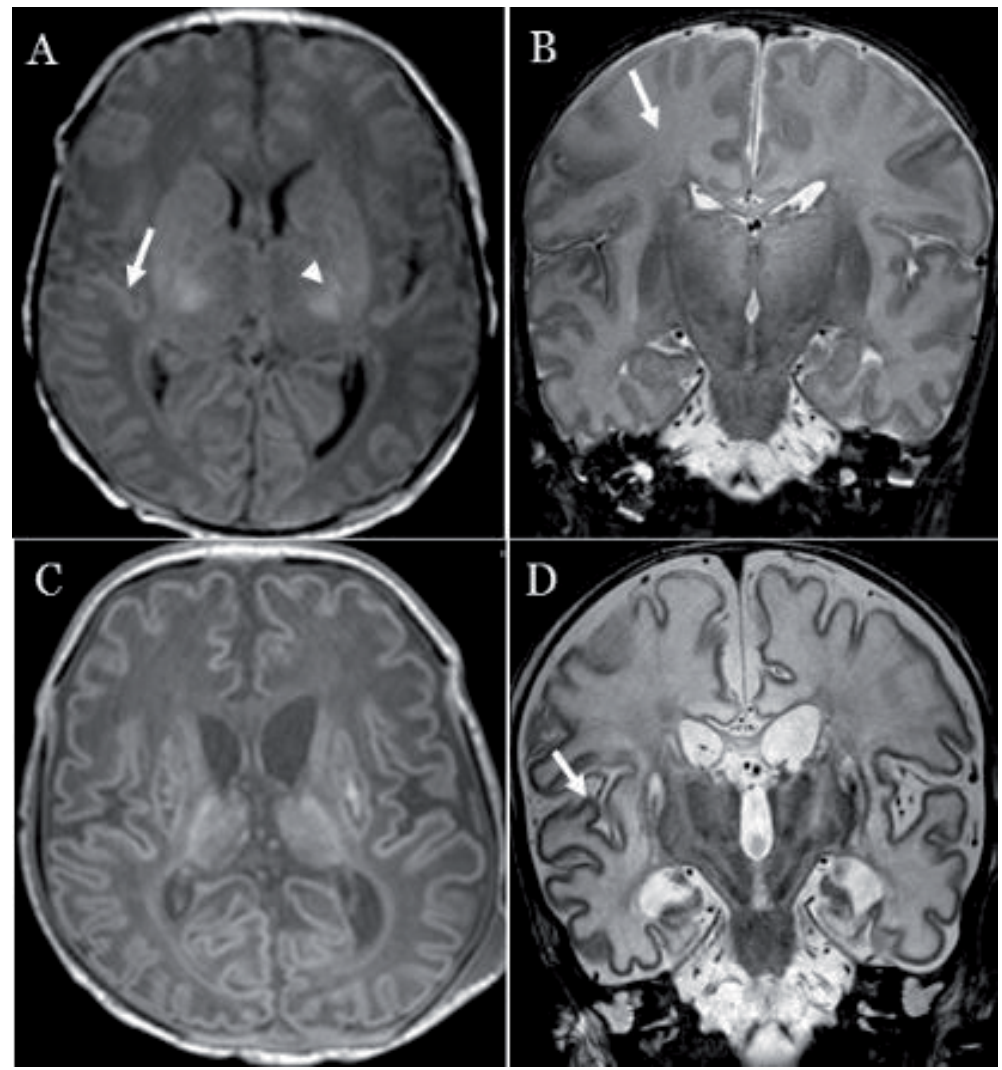

Figure 20.

Images showing the evolution of HIE in a term newborn. In the acute phase, axial T1w section (A) shows hyperintensity of the right Heschl's gyrus (arrow) and mild hyperintensity of the basal ganglia (arrowheads), while the coronal T2w section $(B)$ demonstrates a widely hyperintense white matter (arrow). After 1 week: the axial T1w (C) and coronal T2w (D) sections show chronic changes, with more prominent signal changes in the white matter and basal ganglia and loss of brain matter.

MR studies with DWI sequence are widely used as an early outcome measure after hypothermia.

MR spectroscopy, measuring cerebral metabolites changes, has recently been investigated as a possible tool in predicting neurodevelopmental outcomes in newborn with HIE [40]. In the near future, advanced MR technique might be also used to investigate possible correlation between hypothermia and deafness [41].

In conclusion, the latest neuroimaging techniques play a relevant role in the diagnosis of congenital and acquired disorders underlying SNHL. While advanced MR sequences allow to clarify possible differential diagnoses and to achieve a correct identification of the site of lesion from the inner ear to the auditory cortex, the combination of FPCT and MR undoubtedly support the clinicians in the counseling and management of patient's candidate to CI. 


\section{Author details}

Giorgio Conte $^{1}$, Silvia Casale ${ }^{2}$, Sara Sbaraini ${ }^{3}$, Federica Di Berardino ${ }^{4}$ and Diego Zanetti*

1 Department of Neuroradiology, Fondazione IRCCS Ca' Granda Ospedale Maggiore Policlinico di Milano, Italy

2 Università degli Studi di Pavia, Italy

3 Università degli Studi di Milano, Italy

4 Department of Audiology, Fondazione IRCCS Ca' Granda Ospedale Maggiore Policlinico di Milano, Università degli Studi di Milano, Italy

*Address all correspondence to: diego.zanetti@policlinico.mi.it

\section{IntechOpen}

(C) 2020 The Author(s). Licensee IntechOpen. This chapter is distributed under the terms of the Creative Commons Attribution License (http://creativecommons.org/licenses/ by/3.0), which permits unrestricted use, distribution, and reproduction in any medium, provided the original work is properly cited. (cc) BY 


\section{References}

[1] Conte G, Scola E, Calloni S, Di

Berardino F, Zanetti D, et al. Flat panel angiography in the cross-sectional imaging of the temporal bone: Assessment of image quality and radiation dose compared with a 64-section multisection CT scanner. AJNR. American Journal of Neuroradiology. 2017;38(10):1998-2002

[2] Fatterpekar GM, Doshi AH, Dugar M, Delman BN, Naidich TP, Som PM. Role of 3D CT in the evaluation of the temporal bone. Radiographics. 2006;26(Suppl 1): S117-S132

[3] Piergallini L, Scola E, Tuscano B, et al. Flat-panel CT versus 128 -slice CT in temporal bone imaging: Assessment of image quality and radiation dose. European Journal of Radiology. 2018;106:106-113

[4] Conte G, Caschera L, Tuscano B, Di Berardino F, Zanetti D, et al. ThreeTesla magnetic resonance imaging of the vestibular endolymphatic space: A systematic qualitative description in healthy ears. European Journal of Radiology. 2018;109:77-82

[5] Fitzgerald DC, Mark AS. Sudden hearing loss: Frequency of abnormal findings on contrast-enhanced $M R$ studies. AJNR. American Journal of Neuroradiology. 1998;19(8):1433-1436

[6] Sennaroğlu L, Bajin MD. Classification and current management of inner ear malformations. Balkan Medical Journal. 2017;34(5):397-411

[7] Casselman JW, Offeciers EF, De Foer B, Govaerts P, Kuhweide R, Somers T. CT and MR imaging of congential abnormalities of the inner ear and internal auditory canal. European Journal of Radiology. 2001;40(2):94-104. DOI: 10.1016/ s0720-048x(01)00377-1
[8] Davidson HC, Harnsberger HR, Lemmerling MM, et al. MR evaluation of vestibulocochlear anomalies associated with large endolymphatic duct and sac. AJNR. American Journal of Neuroradiology. 1999;20(8):1435-1441

[9] Hwang M, Marovich R, Shin SS, Chi D, Branstetter BF 4th. Optimizing CT for the evaluation of vestibular aqueduct enlargement: Inter-rater reproducibility and predictive value of reformatted CT measurements. Journal of Otology. 2015;10(1):13-17

[10] Casselman JW, Offeciers FE, Govaerts PJ, et al. Aplasia and hypoplasia of the vestibulocochlear nerve: Diagnosis with MR imaging. Radiology. 1997;202(3):773-781. DOI: 10.1148/radiology.202.3.9051033

[11] Fatterpekar GM, Mukherji SK, Alley J, Lin Y, Castillo M. Hypoplasia of the bony canal for the cochlear nerve in patients with congenital sensorineural hearing loss: Initial observations. Radiology. 2000;215(1):243-246

[12] Choi YJ, Sang YP, Myung SK, Ki JS. The significance of a hypoplastic bony canal for the cochlear nerve in patients with sensorineural hearing loss: CT and MRI findings. Journal of the Korean Radiological Society. 2004;50(4):227-236

[13] Brackmann DE, Hitselberger WE, Nelson RA, et al. Auditory brainstem implant: I. Issues in surgical implantation. Otolaryngology and Head and Neck Surgery. 1993;108(6):624-633

[14] Booth TN, Roland P, Kutz JW, Lee K, Isaacson B. High-resolution 3-D T2-weighted imaging in the diagnosis of labyrinthitis ossificans: Emphasis on subtle cochlear involvement. Pediatric 
Radiology. 2013;50:1584-1590. DOI: 10.1007/s00247-013-2747-5

[15] Ruckenstein MJ, Rafter KO, Montes M, Bigelow DC. Management of far advanced otosclerosis in the era of cochlear implantation. Otology \& Neurotology. 2001;22(4):471-474

[16] Calvino M, Sánchez-Cuadrado I, Gavilán J, Lassaletta L. Cochlear implant users with otosclerosis: Are hearing and quality of life outcomes worse than in cochlear implant users without otosclerosis? Audiology and Neurotology. 2018;23(6):345-355. DOI: $10.1159 / 000496191$

[17] Conte G, Di Berardino F, Sina C, Zanetti D, et al. MR imaging in sudden sensorineural hearing loss. Time to talk. AJNR. American Journal of Neuroradiology. 2017;38(8):1475-1479

[18] Conte G, Di Berardino F, Avignone S, Sina C, Iofrida E, Zanetti D, et al. The "full-blown" MRI of sudden hearing loss: 3D-FLAIR in a patient with bilateral metastases in the internal auditory canals. Neuroradiology Journal. 2018;31(1):39-41

[19] Kim HS, Kim DI, Chung IH, Lee WS, Kim KY. Topographical relationship of the facial and vestibulocochlear nerves in the subarachnoid space and internal auditory canal. AJNR. American Journal of Neuroradiology. 1998;19(6):1155-1161

[20] Mark AS, Fitzgerald D. Segmental enhancement of the cochlea on contrast-enhanced MR: Correlation with the frequency of hearing loss and possible sign of perilymphatic fistula and autoimmune labyrinthitis. AJNR. American Journal of Neuroradiology. 1993;14(4):991-996

[21] Lemmerling MM, De Foer B, Verbist BM, VandeVyver V. Imaging of inflammatory and infectious diseases in the temporal bone. Neuroimaging
Clinics of North America. 2009;19(3): 321-337

[22] Conte G, Lo Russo FM, Calloni SF, Sina C, Barozzi S, Di Berardino F, et al. MR imaging of hydrops in Ménière's disease: All that glitters is not gold. Acta Otorhinolaryngologica Italica. 2018;38(4):369-376

[23] Conte G, Caschera L, Calloni S, Di Berardino F, Zanetti D, et al. MR imaging in Menière disease: Is the contact between the vestibular endolymphatic space and the oval window a reliable biomarker? American Journal of Neuroradiology. 2018;39(11)59:2114-2119. DOI: 10.3174/ ajnr.a5841

[24] Prenzler NK, Bültmann E, Giourgas A, et al. Cochlear implantation in patients with definite Meniere's disease. European Archives of OtoRhino-Laryngology. 2017;274(2):751756. DOI: $10.1007 / \mathrm{s} 00405-016-4356-\mathrm{z}$

[25] Guleria S, Kelly TG. Myelin, myelination, and corresponding magnetic resonance imaging changes. Radiologic Clinics of North America. 2014;52(2):227-239

[26] Moore JK, Perazzo LM, Braun A. Time course of axonal myelination in the human brainstem auditory pathway. Hearing Research. 1995;87(1-2):21-31

[27] Long P, Wan G, Roberts MT, Corfas G. Myelin development, plasticity, and pathology in the auditory system. Developmental Neurobiology. 2018;78(2):80-92

[28] Wang S, Fan G. Alterations of structural and functional connectivity in profound sensorineural hearing loss infants within an early sensitive period: A combined DTI and fMRI study. Developmental Cognitive Neuroscience. 2019;38:100654

[29] Korver AMH, Smith RJH, Van Camp G, et al. Congenital hearing 
loss. Nature Reviews. Disease Primers. 2017;3:16094

[30] Smith RJH, Bale JF, White KR.

Sensorineural hearing loss in children. The Lancet. 2005;365(9462):879-890. DOI: $10.1016 / \mathrm{s} 0140-6736(05) 71047-3$

[31] Manicklal S, Emery VC, Lazzarotto T, Boppana SB, Gupta RK. The "silent" global burden of congenital cytomegalovirus. Clinical Microbiology Reviews. 2013;26(1):86-102

[32] Smiljkovic M, Renaud C, Tapiero B, Lamarre V, Kakkar F. Head ultrasound, $\mathrm{CT}$ or MRI? The choice of neuroimaging in the assessment of infants with congenital cytomegalovirus infection. BMC Pediatrics. 2019;19(1):180

[33] Bonifacio SL, Glass HC, Vanderpluym J, et al. Perinatal events and early magnetic resonance imaging in therapeutic hypothermia. The Journal of Pediatrics. 2011;158(3):360-365

[34] Doneda C, Parazzini C, Righini A, et al. Early cerebral lesions in cytomegalovirus infection: Prenatal MR imaging. Radiology. 2010;255(2):613-621

[35] Kwak M, Yum M-S, Yeh H-R, Kim H-J, Ko T-S. Brain magnetic resonance imaging findings of congenital cytomegalovirus infection as a prognostic factor for neurological outcome. Pediatric Neurology. 2018;83:14-18. DOI: 10.1016/j. pediatrneurol.2018.03.008

[36] Severino M, Zerem A, Biancheri R, Cristina E, Rossi A. Spontaneously regressing leukoencephalopathy with bilateral temporal cysts in congenital rubella infection. The Pediatric Infectious Disease Journal. 2014;33(4):422-424

[37] Steinlin MI, Nadal D, Eich GF, Martin E, Boltshauser EJ. Intrauterine cytomegalovirus infection: Clinical and neuroimaging findings. Pediatric
Neurology. 1996;15(3):249-253. DOI: 10.1016/s0887-8994(96)00170-1

[38] Groenendaal F, de Vries LS. Fifty years of brain imaging in neonatal encephalopathy following perinatal asphyxia. Pediatric Research. 2017;81(1-2):150-155

[39] Fitzgerald MP, Reynolds A, Garvey CM, Norman G, King MD, Hayes BC. Hearing impairment and hypoxia ischaemic encephalopathy: Incidence and associated factors. European Journal of Paediatric Neurology. 2019;23(1):81-86

[40] Shibasaki J, Aida N, Morisaki N, Tomiyasu M, Nishi Y, Toyoshima K. Changes in brain metabolite concentrations after neonatal hypoxicischemic encephalopathy. Radiology. 2018;288(3):840-848

[41] Lucke AM, Shetty AN, Hagan JL, et al. Early proton magnetic resonance spectroscopy during and after therapeutic hypothermia in perinatal hypoxic-ischemic encephalopathy. Pediatric Radiology. 2019;49:941-950. DOI: $10.1007 / \mathrm{s} 00247-019-04383-8$ 



\title{
Audiology's Third Pillar: Comprehensive Follow-Up Care and Counseling for Those Who Choose to Self-Direct Their Care
}

\author{
Brian Taylor
}

\begin{abstract}
Given the anticipated changes to hearing aid distribution, it is imperative for hearing care professionals to identify new approaches to providing services for persons with hearing loss. One of the primary challenges associated with over-thecounter and self-fitting hearing aids is that all of the control is placed into the hands of the consumer. Although a considerable number of aging Baby-boomers prefer to self-direct their healthcare, when provided that option, a relatively small number of persons with hearing loss are likely to successfully complete the entire process of self-assessment, and self-fitting of hearing aids. Building on the two cornerstones of clinical audiology: diagnosis in the medical arena and rehabilitation in the delivery of services for individuals with chronic conditions, this chapter proposes the new, third pillar of audiology is the provision of comprehensive follow-up and counseling. Moving beyond simply instructing persons with hearing loss on how to use their hearing aids, this chapter lays the groundwork on guiding individuals on becoming independent self-managers of their condition and how these services can be provided in an ethical and profitable manner in a typical clinic.
\end{abstract}

Keywords: self-directed care, patient decision aids, patient centered communication, self-fitting hearing aids, shared goal setting, device mastery skills, self-management skills, service packages

\section{Introduction}

By most standards, audiology is a relatively young profession, burgeoning in North America and Western Europe following World War II. Cutting its teeth by managing the needs of soldiers returning from the war, audiology centered on two distinct practices: (1) Diagnostics, particularly the identification of ear diseases using an evolving battery of site of lesion testing. This battery of tests included, air and bone conduction pure tone threshold testing, speech audiometry, tympanometry, and acoustic reflex thresholds. Eventually, electrophysiologic assessment of the auditory and balance system including auditory brainstem response (ABR) and otoacoustic emissions (OAE) assessment were added to armamentarium of audiologists involved in diagnostic assessments. (2) Treatment and rehabilitation, specifically the selection and fitting of hearing aids for individuals with 
benign forms of hearing loss. Aural rehabilitation, with its emphasis on exercises to improve communication skills, should remain an important part of the second pillar. Beyond these traditional components of Audiology, changes in the market are likely to provide opportunities to provide different types of services to individuals with hearing loss.

Managing adults with hearing loss is by far the most fundamental aspect of audiology practice making this the bread and butter of our profession. Both pillars of clinical audiology, diagnostics and treatment/rehabilitation, will remain integral components of care for persons with hearing loss, however, technological progress, driven mainly by Moore's Law and the evolving consumer demands of the Baby-boomer generation are expected to change how Audiology is practiced. Over the next decade, self-fitting hearing aids and other amplification devices purchased over the counter, without the assistance of a licensed professional, will likely enable persons with hearing loss to self-direct or self-manage their own care. The availability of self-directed care, which includes self-administered hearing testing and self-fitting hearing aids, will enable persons with hearing loss to select and fit hearing aids without intervention of an audiologist. The ability of persons with hearing loss to self-direct their care warrants the development of a third pillar in Audiology. One devoted to comprehensive care and well-being of the person with hearing loss that is not dependent on the purchase of hearing aids directly from a licensed professional.

Self-directed or self-managed care is defined as a patient's or customer's ability to identify and treat a perceived condition without the assistance, guidance or input of a credentialed expert. The purchase of medication, such as pain relievers for a headache is perhaps the simplest type of self-directed care. Smartphone-enabled apps that collect and analyze bodily functions are allowing more opportunities for people to self-direct their own care. Self-fitting hearing aids may soon be a viable option for individuals opting to self-direct their hearing care. If these types of devices are purchased on-line, a growing number of individuals could seek services from an audiologist after they have purchased hearing aids elsewhere.

\section{Self-fitted hybrid hearing devices}

Hearing aid technology has never been better, yet a surprisingly large number of persons with hearing loss fail to embrace it. Fortunately, as smartphoneenabled apps, Bluetooth streaming and voice-activated algorithms find their way into traditional hearing aids, it increased the chances that this technology can be successfully selected and fitted without the guidance of an audiologist. Exactly who can benefit from this newer technology has not been firmly established, nevertheless, audiologists must be ready to practice in a future where some people can self-fit their hearing aids, referred to in this chapter as self-fitted, hybrid devices.

The combination of changing demands within the market, led by an aging Babyboomer population and rapidly evolving hearing aid technology, has paved the way for new ear worn products. These products combine the advantages of traditional hearing aids, such as stable gain without feedback and sophisticated noise reduction technology with consumer audio products that interface with smartphone-enabled apps and allow their users to easily adjust them, often with voice-activated technology. Commonly referred to as hybrid hearing devices, there are a range of products that exist on a continuum. Some of these products are classified as traditional hearing aids, while others are conventional consumer audio products that happen to provide some nominal amount of amplification. 
Importantly, both hearing aid manufacturers and consumer electronics companies are bringing products to market today that are considered, multi-tasking hybrid devices. Some, of course, have as their core function amplification with direct audio streaming via Bluetooth or biometrics as a secondary function. On the other hand, consumer electronics companies are bringing a wide range of ear worn devices to market that have customizable amplification as one of many features for the wearer to use. As technology continues to evolve, especially in the emerging era of voice activated algorithms (e.g., Siri and Alexa), these hybrid devices are likely to become easier to use for a wider range of the population.

As we move into this brave new world of multi-tasking audio gadgets, here are a few key points to remember about these hybrid devices: (1) These are true multi-tasking products that provide the wearer with a core function, which could be amplification, phone and music streaming, language translation or biometrics, and whatever feature from this list not deemed by the wearer as the core feature becomes a secondary function for the wearer. (2) Many of these devices are selffitting in nature. This, in theory, enables their wearer to purchase the device, finetune and wear it without ever seeing an audiologist. (3) Some, yet to be determined number of individuals, who are interested in these hybrid devices will need help navigating the myriad choices, or after they have purchased a hybrid device will need some expert help and attention on maximizing their use-this final point is the third pillar of Audiology.

\section{The third pillar: comprehensive follow-up care and counseling}

The ability to self-direct hearing care means that a potentially large number of individuals with hearing loss may not have to see an audiologist for care until after they have purchased hearing device. Given that most hearing aid purchases today are completed in a bundled manner (hearing aids and services are sold together as one "package"), the advent of self-fitting hearing devices will present Audiologists with the challenges of offering more unbundled professional services to individuals with hearing loss that purchased devices elsewhere, but now need counseling or device management support.

Some Audiologists are already offering "unbundled" or "itemized" hearing care services. This may be to cope with the constantly changing needs of individuals with hearing loss and to differentiate themselves in an evolving healthcare marketplace. Such an approach may bring opportunities for Audiologists to increase market share by assisting persons who may have purchased hearing devices online without the audiological services or support. However, little has been written about the ways in which Audiologists can effectively offer decoupled audiological services. The purpose of this chapter is to provide guidance and insights on how a third pillar of Audiology, one firmly centered on comprehensive follow-up care and counseling, unbundled from the sale of hearing aids, can be used to better serve the community and generate revenue for practice owners. Before offering specific comprehensive follow-up care and counseling strategies, let us take a closer look at how audiological services have been customarily delivered and how that is likely to change.

\subsection{Units based versus time-based business models}

For more than 30 years, regardless of practice setting, most audiologists have generated much of their practice revenue from the dispensing of hearing aids. The generation of this revenue is predicated on the number of hearing aid units sold over any given time frame. Given the large profit margins historically associated 
with the commercial sale of hearing aids, a relatively few number of hearing aid units could be dispensed within a month for a practice to remain profitable. For example, survey data collected from reputable sources, such as MarkeTrak 9 [1] that for every full time licensed professional working with a practice, 15-20 hearing aids per month are dispensed.

A relatively few hearing aids dispensed per month to maintain profitability is a basic descriptor of a units based business. In a units based business, marketing plans and operational strategies are implemented in the clinic with one primary objective: Reach a "units sold" target which covers all costs and generates a marginal profit for the practice. Stated differently, it does not matter too much how many patients you see in your clinic, if the hearing aid units sold number is sustained, the business goals are achieved.

The units based business model is summarized in Figure 1. The schematic shows the three key drivers of a productive hearing aid dispensing practice. Office traffic, which is a primary function of marketing and branding, is designed to bring enough people to the clinic that pre-defined number of units sold (hearing aids) can be achieved at a retail price (ASP = average selling price) that is appealing to patients and dispensed at a per unit margin that is profitable for the practice.

The prior statements are not intended to denigrate the unit-based business model. Unit-based business models are beneficial to consumers and clinicians. The high margin associated with the dispensing of hearing aids is largely a by-product of the number of hours it takes to select, fit and fine tune hearing aids, along with the substantial amount of face-to-face counseling time need to orientate and educate patients in the routine use of hearing aids.

The downside to the units based business model, however, is that it is inefficient. Because the primary objective of a units based business is to achieve a specific "unit sold" target each week or month, the model tends to view all patients in the same binary way: The patient is either a hearing aid sale opportunity today or a hearing aid sales opportunity in the future. And, revenue is generated only on those that are sales opportunities today. This binary view of patients - either the patient is a sales opportunity today or in the future-is at the heart of the inefficiency of

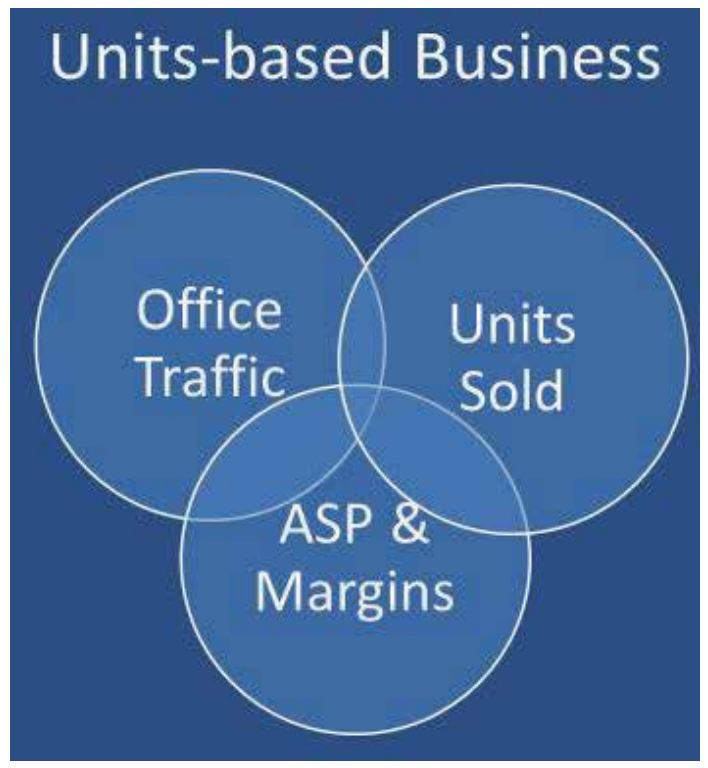

Figure 1.

The three key components of a units based audiology or hearing care business. ASP = average selling price. 
the units based business. It can take an inordinate amount of time to determine which category a patient falls into and when that determination is finally made, only the patients who agree to purchase hearing aids today generate revenue for the practice. Oftentimes many of the patients seen in a clinic, who might be candidates for hearing aids, generate zero revenue if they do not purchase hearing aids-even though a clinician spent an hour or more with the patient conducting a hearing test or hearing aid consultation.

Additionally, the binary units based business model in which revenue is generated only when hearing aids are sold contradicts a lot of what we know about the psychosocial aspects of hearing loss in adults. A relatively large subsegment of persons with hearing loss seek the advice of audiologists even though they may not be ready to move ahead with a treatment plan that includes hearing aids. Although these patients likely benefit from a professional encounter with a clinician who helps them sort through the psychosocial aspects of hearing loss, it is common practice for these patients to not be billed for time spent with the audiologist unless hearing aids are purchased.

\subsection{Unbundling and Itemizing}

Unlike many technical terms in the profession of audiology, these two terms are not officially defined, so they are a little fuzzy. Here is one common way to differentiate these two terms: Itemizing refers to a list of all the services and procedures completed by a professional. Even though a charge might be associated with each procedure that is itemized, the patient typically pays a single fee that is bundled with the service. On the other hand, unbundling refers to separate fees associated with various services delivered during an appointment. For example, it is becoming more common in the United States for audiologists to charge one fee for the hearing aids and another fee for professional services associated with provision of those devices. Most audiologists would consider this example an unbundled service model.

In contrast to a unit-based business, a time-based business places monetary value on virtually all encounters between the patient and the provider. Revenue is generated in a time-based business even when hearing aids are not dispensed to the patient receiving the services. There are several forces dictating that audiologist move from a units based to more of a time-base business model. The root cause of these market forces is the rapidly aging senior population. It is believed approximately 10,000 aging baby-boomers are turning 65 years of age each day. Given the size of their demographic and the prevalence of hearing loss associated with age, third party health insurance companies (Medicare Advantage programs), big-box retail chains and start-up consumer electronics companies are poised to tap into this growing demand for novel approaches to hearing care. The opportunity looks even riper when low hearing aid ownership rates among older Americans with hearing loss is considered.

Figure 2 shows an example of the underpinnings of a time-based business. Notice office traffic is still a primary driver of revenue-generating activity; however, as tele-health becomes a reimbursable option of service delivery, some of types of face-to-face encounters could be replaced by it. Also, notice in the time-based model, "units sold" is replaced with "revenue generating procedures." As more third party payers and direct to consumer hearing aid options come to market, clinicians can expect a growing number of patients to bring an already-purchased hearing aid that require some type of service. Additionally, in the future, it is plausible that clinicians, after a careful triaging process, could recommend patients purchase selffitting hearing aids at a lower cost. Patients that opt to self-fit could then purchase services from the audiologist. 


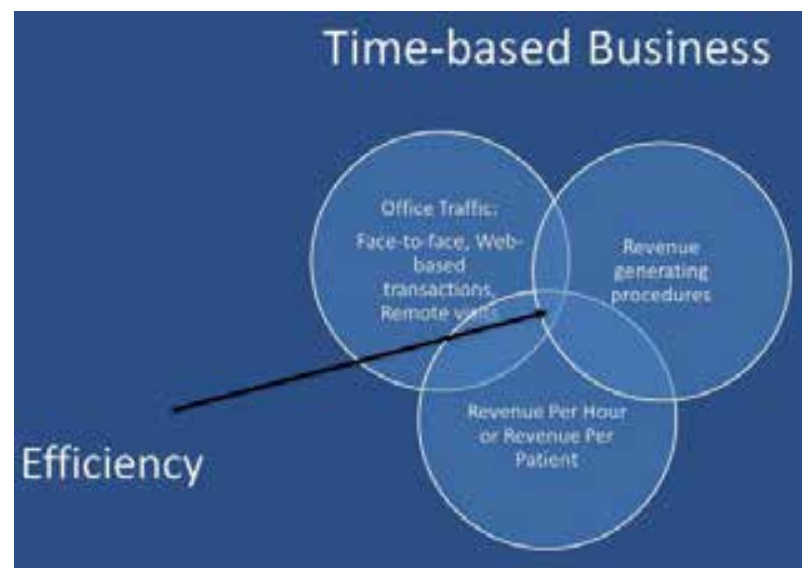

Figure 2.

The key characteristics of a time-based business model.

It is unlikely the time-based business model will completely supplant the units based model. After all, a substantial number of patients will still seek to purchase their hearing devices from a licensed professional. For rank and file clinicians, however, working in a units based business model that generates most of their revenue through the sale of hearing aid units, a substantial amount of money could be left on the table when a growing number of older Americans might purchase hearing aids directly from a Medicare Advantage program, a big-box retail center or on-line. Thus, the ability to offer a service of value-one that stands apart from the delivery of a product-is imperative to the financial viability of the practice in the emerging era of consumer-driven hearing care. Given the importance of time in the revenue generating process, combined by the fact that the number of available clinical hour is restricted to six or seven patient appointments per day, efficiency is of paramount importance.

A cornerstone of any time-based business is filling the audiologist's schedule with as many revenue generating opportunities as possible. This requires careful management of the schedule to ensure there is ample time to see new patients without them having to wait more than a few weeks to see the professional for an appointment. In an audiology business, the necessary follow-up service needs of existing patients always threaten to overtake the time of the professional who needs to have new revenue-generating opportunities on their schedule. By following the three-step approach below, licensed providers can embrace a time-based business model without compromising the profitability of the units based business model.

In a time-based business model, it is the amount of time needed-not the number of procedures you conduct, or the number of hearing aids (units) dispensed-to optimize patient outcomes that matters the most. If you are moving from a units based business model to one that is primarily time-based, there are two important considerations. Each consideration warrants careful attention and planning on the part of the clinical manager.

\subsubsection{Determine revenue per hour}

In a time-based business, it is critical to know how much to charge for time spent with patients. This requires calculating revenue per hour (RPH) of the practice. The main elements of the RPH equation are gross profit requirements and available productive hours. To determine $\mathrm{RPH}$, the numerator of the equation is gross profit requirements, which are generally considered to be gross revenue, cost 


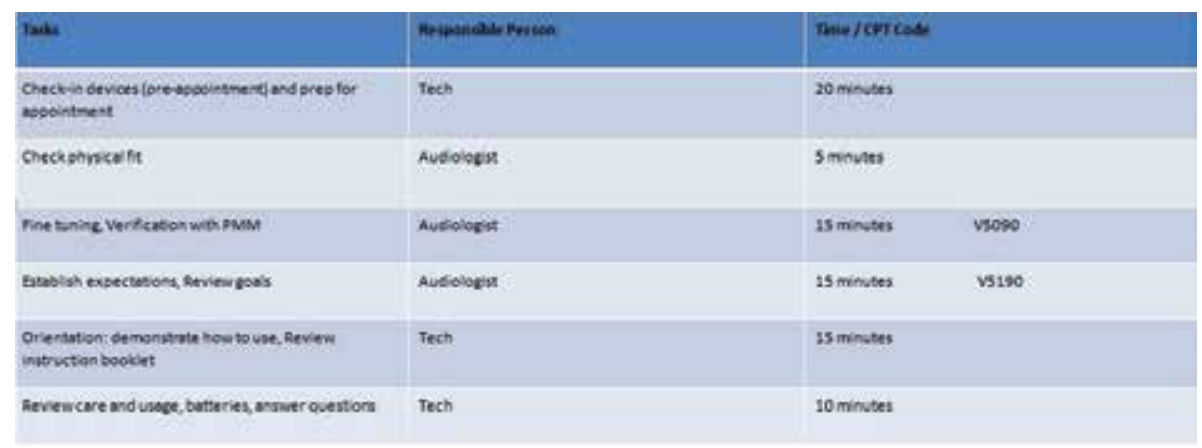

Figure 3.

An example of how the labor required to meet the needs of one person with hearing loss is divided between the audiologist and the technician/assistant.

of goods of hearing aids and an expected profit margin. The denominator of the equation is available productive hours, which are the total number of hours calculated over an entire year for one full time audiologist to see patients. Typically, this number is based on a full-time clinician seeing patients for 6 hours per work day. Once you have gathered all those numbers, the RPH calculation is simple. In many cases, the RPH number is around \$200-250 (American dollar) per hour.

The RPH number that is calculated for a practice is used to determine timebased pricing. For example, if the RPH number is $\$ 200$, then a 30 -minute appointment needs to have a fixed price of around $\$ 100$. Just as important as determining pricing for time-based service appointments, the RPH value helps determine if your practice should sign-up for a third party insurance contract. For example, let us say, there is a Medicare Advantage program willing to pay you an $\$ 800$ fitting fee for a pair of hearing aids with standard, mid-level technology. As part of the service contract, your practice is expected to provide three visits, which includes the initial fitting. If your RPH is $\$ 200$, this is a profitable transaction because the fitting fee of $\$ 800$ exceeds the minimum amount of time required to spend with the patient. The challenge, of course, with this type of time-based service agreement is that some patients require considerably more time if their needs are more complex.

\subsubsection{Define service packages}

Once revenue per hour (RPH) has been calculated, the next step is to determine what tests and procedures will be included in various service packages. Before we delve into the details of service packages that can be offered in a typical audiology practice, later in this chapter, it is important to note that whatever service package is defined and created by a practice must stand alone from the delivery of a device. That is, one purpose of the service package is to offer something of value to the individual who purchased devices elsewhere but needs additional intervention or assistance. Figure 3 shows how time spent with a person with hearing loss can be divided between the licensed audiologist and a technician who assists the audiologist in the delivery of care. Note how labor is divided between the two professionals.

\section{Two types of help seekers}

To better appreciate the delivery of an unbundled service package, let us examine two different types of help seekers who are apt to find their way into an 
audiology clinic in an era in which persons with hearing loss can choose to selfdirect their care. In a marketplace where individuals have the option to self-direct their care, audiologists must be prepared to offer services to two distinct types of patients: (1) help seeking individuals who do not own hearing devices and (2) individuals who own hearing devices seeking help from a professional. Additionally, it is worth acknowledging there will be some patients who will choose to self-direct their care who will not ever see an audiologist, either because they are successful in the self-fitting process and do not need the help audiologists provide or have been unsuccessful and have given up on the process.

For help seeking individuals who do not currently own hearing devices, a primary objective of the communication assessment, which is conducted by audiologists, is to separate patients who view their condition of low importance from those who view their condition to be of high importance. A basic tenet of patient-centric care is ensuring persons with hearing loss have a choice as to when they desire to begin treatment. Knowing if the person with hearing loss believes the condition is important enough to begin treatment is a critical initial step. Thus, during an initial appointment in which communication needs are assessed, it is critical to ask about the importance (or urgency) to treat their communicatively significant hearing loss.

\subsection{Help seekers without devices: importance to treat is a touchstone}

In a world where people can choose to self-direct their care, their pathway to the audiology clinic is likely to be different than how individuals currently receive hearing care services. Historically, individuals with hearing loss completed all the tasks related to hearing aid purchase and use from a single clinic. Today, it is becoming apparent that hearing devices can be purchased without the assistance of a licensed hearing care professional from one entity (e.g., Amazon or Hearing Planet) and fitted and adjusted elsewhere, if the buyer of the hearing device seeks additional help. It stands to reason that many of these individuals seeking help for their hearing loss that do not own hearing aids, could independently visit a clinic, retail shop or even a website and evaluate a range of hybrid products. (A hybrid device combines features of a traditional hearing aid [e.g., customizable gain settings, frequency shaping] with features commonly found in a consumer electronic device [e.g., voice-activated algorithms, music streaming]).

Moreover, over-the-counter (OTC) hearing aids are expected to become a regulated medical device by the U.S. Food and Drug Administration (FDA) by the end of 2020. Thus, a growing number of American consumers could purchase amplification devices without first seeking input or guidance from a state licensed hearing care professional and for various reasons eventually consult a licensed professional with hearing devices in hand. To prepare for a future where OTC hearing device exist as an officially regulated medical device, and to remain an integral part of providing professional services in a marketplace filled with hybrid devices, audiologists need to become well-versed in helping people choose the hybrid product that is right for them.

The bottom line is clinicians must realize individuals with hearing loss can purchase amplification devices directly without the assistance of a licensed professional. As these devices become more readily available (and likely more user friendly with better sound quality), clinicians must identify consumer "pain points" where they can add value. The next section examines ways clinicians can add value, decoupled from the sale of a product.

\subsection{Use of decision aids}

Clinicians are encouraged to use easy to read decision aids that depict a range of hearing devices and treatments, from personal sound amplification products 
(PSAPs) to hearing aids with direct streaming. The role of the audiologist is to summarize the pros and cons of each category of treatment in relation to the needs and test results of the individual in need of services.

Let us examine a more traditional example of a person in need of help from an audiologist and how patient centric tools can be applied to the process of guiding patients through the process of improving their communication ability. Most clinicians probably encounter the following situation more than a few times per year: A older adult with a moderate, bilateral hearing loss-an audiogram crying for help, who perceives the problem to be "no big deal" or believes his hearing is near-normal. Even for the patient with significant hearing loss who is a hearing aid candidate, if the problem is considered by that patient to be of low importance to treat, spending an hour convincing them to try hearing aids is usually an ineffective tactic. On the other hand, for patients who view their condition to be of high importance to treat, spending an hour or more with that patient is more likely to result in a set of well-planned treatment goals and the purchase of hearing devices. To separate patients of low importance from those that consider their condition to be of high importance, the use of a simple scaling question, "On a scale of 1-10, 10 being the most important priority for you today and 1 not important at all, how important is getting help for your hearing loss?" is extremely helpful. It is important to remember patients with a chronic condition need to buy-in to the treatment process for it to be effective, therefore, understanding the patient's perspective is paramount. Asking about how convinced they are that their condition is important to treat is a useful starting point.

For the "importance to treat" question, if the patient provides a number lower than, say, six, it is an indication that the patient's awareness of their condition and its impact on daily activities needs to be raised. When patients who view their condition to be of low importance to treat it does not make sense to convince them to accept a recommendation of hearing aids_-even when significant hearing loss is present. Rather, the focus of the initial appointment with the audiologist should be to increase patient awareness of the consequences of their condition on daily communication.

This process begins at the initial appointment, but treatment may not begin for some time later when the patient is ready to move forward with treatment because their communication deficit becomes more important for them to treat. Thus, patients who view their condition to be of low importance to treat usually require less face-to-face time with the clinician at the initial appointment and should be encouraged to schedule another appointment later (perhaps 3-6 weeks) to monitor the patient's perception of the condition and their willingness to move ahead with goal setting and treatment planning.

For patients who view their condition to be of low importance to treat, rather than discussing treatment options, such as hearing aids, the audiologist can ask the patient to thinking about places where communication is becoming a burden. Part of this dialog can be a discussion of the emotions associated with an inability to communicate effectively. The communication partner, typically a spouse, should also be involved in this conversation. The goal of the conversation between patient, communication partner and audiologist are to raise awareness of the impact their condition has on communication.

One tactic to consider employing with patients who view their condition to be of low importance to treat is the use of a journal or log. Figure 4 is an example of a check list that can be used to raise awareness of the consequences of untreated hearing loss and its effect on mood and behavior. The idea is to provide this checklist to the patient and communication partner at the end of their first appointment and ask them to complete the check list within the next 3 weeks and return for a second, 

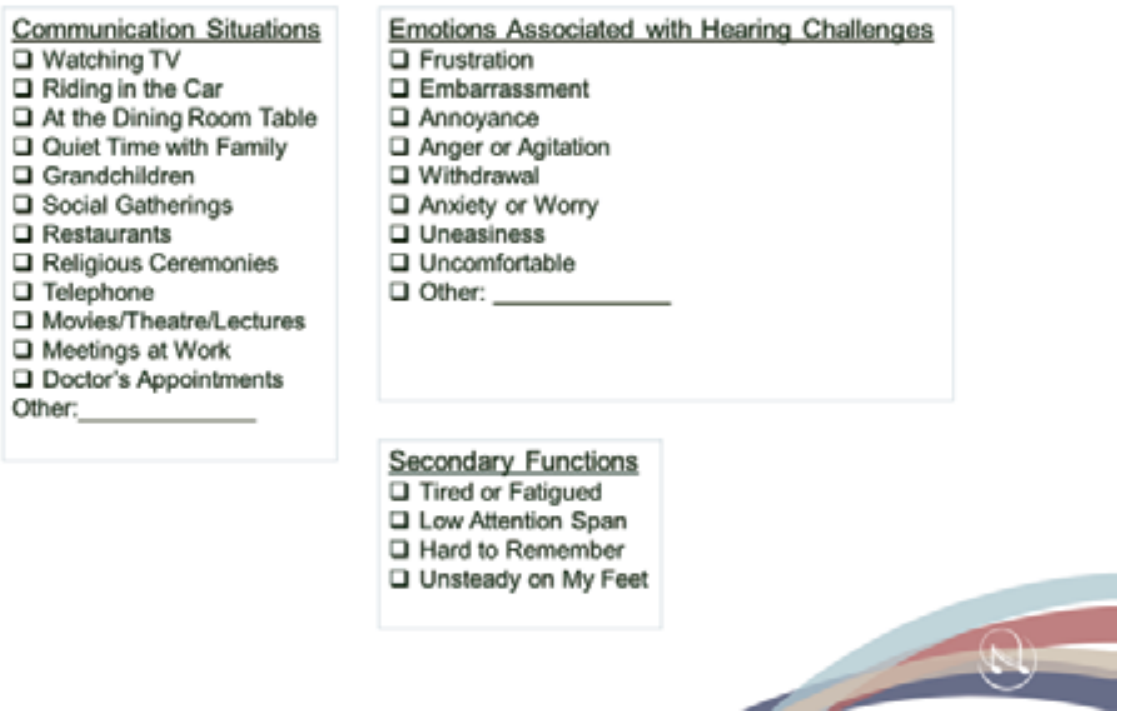

Figure 4.

An example of a simple checklist that helps raise awareness of consequences of untreated hearing loss. With guidance from the audiologist, a patient along with a communication partner completes this prior to a second appointment.

exploratory appointment to discuss the results and the need to move forward with treatment.

Much is still to be learned about the best approaches to working with persons with hearing loss who view their condition to be of low importance to treat. Currently, in the units based business model in which revenue is generated only when hearing aids are dispensed and services are bundled into the price of each sale, persons with hearing loss who view their condition as low importance to treat receive professional services, but often receive those services for free. For first time help seekers without hearing aids, when this initial appointment is viewed as "counseling time" in which the clinician is guiding the patient through the selfdiscovery process using effective information gathering tactics and exploratory dialog, it might be perceived by patients as a high value service that warrants a nominal fee. Additionally, it is possible that patients who perceive their condition to be of low importance to treat might be amenable to a use of low cost PSAP as a lowrisk starter device. In a recent University of Iowa study that used realistic listening conditions, the researchers found that while three the PSAPs evaluated in the study did not outperform professionally fitted hearing aids, the PSAPs did offer significant improvement compared with the unaided condition, and thus could serve as a budget-friendly option for those who cannot afford or do not want to try traditional hearing aids.

Contrast individuals with low importance to treat with those who consider their hearing loss to be of high importance to treat. It is likely these patients would provide a much higher rating on the scaling questions posed above, say a seven or higher on the scale, and therefore require more time with the audiologist during an initial appointment. During this initial appointment treatment goals could be targeted, and agreement could be reached on a treatment plan, usually involving hearing aids. Figure 4 is an example of a goal planning sheet, called the patient expectation worksheet (PEW). Note there is space to customize five specific treatment goals. Additionally, the goal planning sheet can be used to compare the pre-treatment expectations of the patient to the pre-treatment expectations of 
the audiologist. The dialog generated from the tactic of comparing views on pretreatment expectation is a great example of how a patient, who is an expert on their own personal needs and lifestyle demands and an audiologist, who is an expert on hearing disorders and treatment options, work together to arrive at a workable solution. The PEW is also used following treatment to gauge the success of treatment.

Regardless of a patient's self-rating on the importance to treat scale, the time spent with the patient by the audiologist warrants a fee. For the patient who selfrates low on the importance to treat scale, a 30-minute appointment might be sufficient, while the patient who self-rates higher on the importance to treat scale is apt to require one full hour of time during which time several variables such as speech understanding in noise ability, motivation, family support, self-efficacy, and other factors are assessed.

Besides using the importance to treat scaling question to guide the flow of an appointment, audiologists need to identify mechanisms that accurately identify patients who might be able to successfully self-fit hearing aids. Many of the controls used by audiologists to program and fine tune hearing devices will be handed over to patients via a smartphone app. Thus, in the future, audiologists need mechanisms in place that help them separate who are good candidates for self-fitting hearing aids from candidates for audiologist-driven, conventional hearing aids.

One of the central tenets of patient centered care is that the patient and provider collaborate in the goal setting and treatment planning process. Both parties bring something important to the help seeking appointment and it is the responsibility of the audiologist to articulate the valuable role both parties play during this process: The patient is the expert on their condition and the audiologist is the expert on hearing loss and treatment options. This partnership comes to fruition during the goal setting process. As noted in Figure 5, the Patient Expectations Worksheet (PEW) allows the patient to articulate treatment goals and to self-rate their ability to communicate in those areas targeted for improvement on a 1 (hardly ever) to 5 (almost always) scale. This is noted by the letter ' $\mathrm{C}$ ' on the PEW. Next, the patient is asked to self-rate on the same 1-5 scale where they expect to communicate post treatment. This is signified on Figure 5 with the letter ' $E$ '. After the patient has provided these two self-ratings, the audiologist then provides, based on their interpretation of the patient information gathered during the assessment process, their prognosis for realistic improvement following treatment. This is signified on Figure 5 with the checkmark. Finally, the letter 'I' is used to connote the actual level of improvement 3-6 weeks following intervention.

\section{PEW - post-treatment}

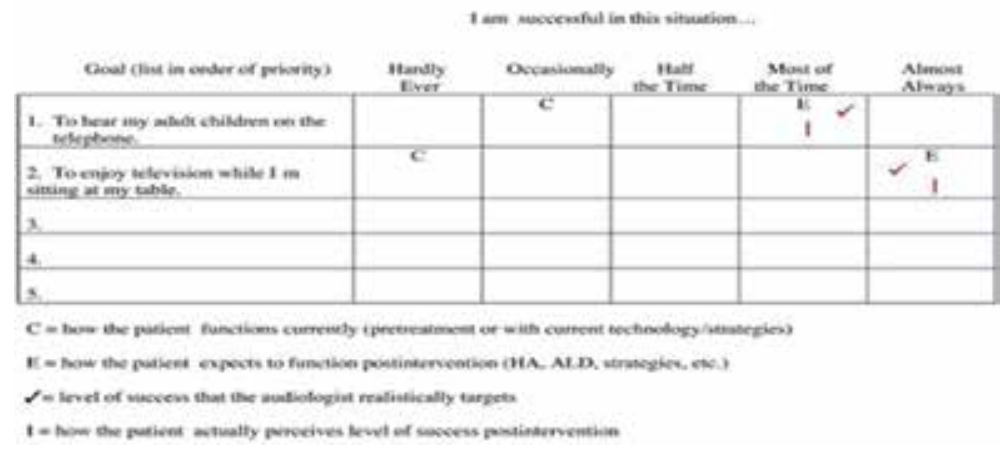

Figure 5.

The patient expectation worksheet (PEW) used to target individualized treatment goals and expectations. 
Figure 6 shows an alternative shared goal setting approach involving input from the patient's communication partner. Developed by Jill Preminger and others associated with the Ida Institute, The GPS (Goal sharing for Partner S) is a step-bystep guide designed to facilitate discussions between the person with hearing loss and their communication partner to establish common communication goals. As stated on the Ida Institute website, the purpose of GPS is to help the person with hearing loss and the communication partner to: (1) acknowledge the hearing loss and the activity limitations and participation restrictions placed on each by the hearing loss and the resulting emotional impact; (2) recognize their communication partnership and accept their shared responsibility to work together to improve communication; and (3) establish realistic communication goals and determine the steps necessary to achieve these goals.

The GPS allows the person with hearing loss and communication partner to first identify areas of daily life when communication may be easier. Next, both parties identify communication problems each experiences and then each person is invited to take the perspective of the other person and identify communication areas each thinks the other person is finding to be a problem. Finally, each party is invited to collaborate on some shared goals.

\subsection{Individuals with hearing devices seeking help}

Unlike help seekers who do not own hearing devices, the second category of patients, help seekers who own hearing devices, is not commonly seen in clinics today, but their numbers are expected to grow. With the expected launch of selffitting hearing aids (SFHAs) and other direct to consumer hearing devices, this second category of patients could benefit from service provided by an audiologistafter they have already purchased SFHAs or another type of amplification device purchased over the counter. The type of help they are seeking could take many forms, however, a couple of recently published studies might shed some light on the role audiologists play in providing services directly to individuals who choose to self-direct their hearing care.

Humes et al. [2] used conventional multichannel hearing aids in a randomized, blinded study that compared the device across three different conditions:

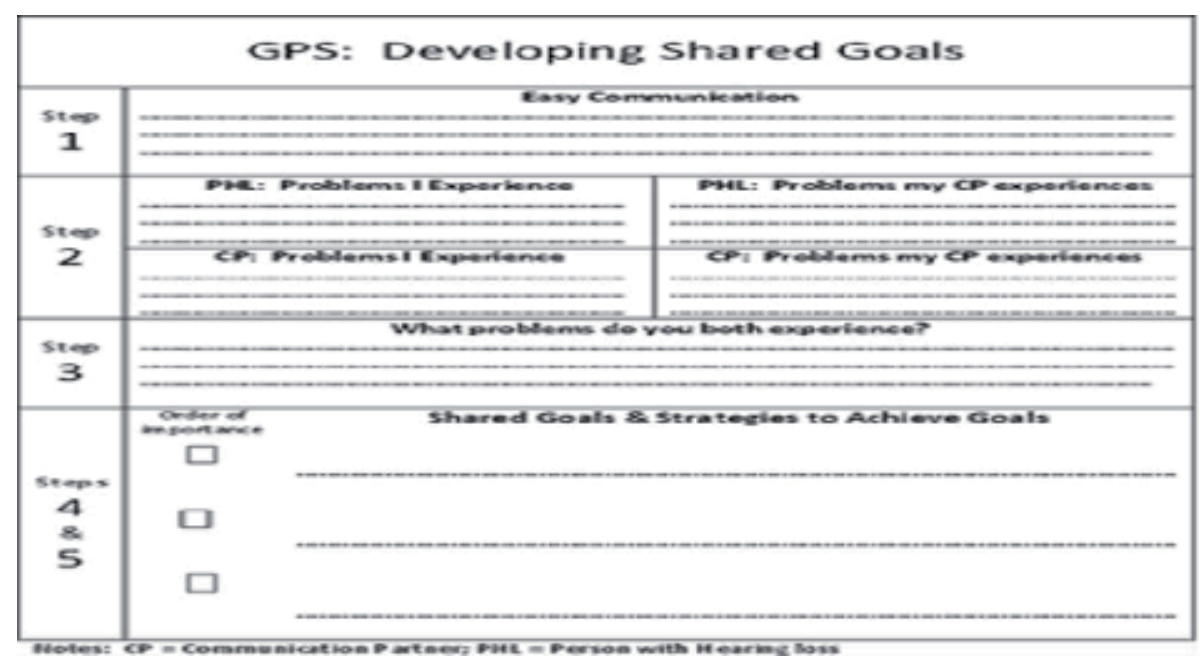

Figure 6.

The goal sharing for partners-S, developed by the Ida Institute. 
(1) Hearing aids pre-set to mimic devices sold over the counter, (2) Professionally fitted devices that matched a prescriptive target and included face-to-face guidance and support from a clinician, and (3) A placebo control in which the hearing aids were set to match the characteristics of the open ear canal. Among the key findings was that $20 \%$ of those fitted with the OTC-like devices benefited from help by the audiologist during the evaluation period. Following the intervention by the audiologist, of the patients who requested help with their OTC-like fitting, approximately half of this group wanted to keep their hearing aids at the end of the evaluation period. These results indicate that a substantial number of individuals, approximately $20 \%$ according to this study, who first opt to self-direct their care by purchasing OTC hearing aids would benefit from the assistance of an audiologist during the initial 30-60 days of device use.

In another recent study examining factors associated with self-fitting hearing aids, Keidser and Convery [3] asked a group of 60 middle-aged to older adults to follow a 9-step task to self-fit a pair of hearing aids. The self-fit hearing aids used in the study were receiver in the canal devices programmed and adjusted with a smartphone app. Additionally, part of the self-fitting task involved an in-situ hearing test.

Several variables, including cognitive status, self-efficacy, problem solving ability, and locus of control that could have impacted participants' success with the self-fitting hearing aid process were evaluated. Results showed that $68 \%$ of the study participants were able to successfully complete the entire self-fitting process either independently or with the assistance of a trained non-audiologist. Of the group that could successfully self-fit, $37 \%$ of them did so independently, while 63\% sought help from the non-audiologist assistant. Two variables, locus of control and problem-solving ability had some limited predictive value, suggesting that both traits should be evaluated before someone purchases self-fitting hearing aids. More interestingly, study participants who did not use a smartphone were more likely to need assistance with the self-fitting process, suggesting that smartphone use is a lead indicator of SFHA candidacy. Finally, those that did need assistance with the self-fitting process received effective help from a nonaudiologist assistance.

Together, these two studies, even though they used slightly different over the counter (OTC) hearing aid delivery models, indicate a self-fitting device should provide access to trained support personnel that can assist the patient with the self-fitting process. And, this support service can be provided successfully by nonaudiologist either in a face-to-face manner or using video conferencing tools, such as Skype.

\subsection{Who might fail at self-fitting hearing aids?}

Assuming self-fitting hearing aids will be purchased over the counter soon, it is important to think about individuals who might try and fail. One group that comes to mind is older adults who have concerned children or grandchildren that buy devices on-line. Audiologist need to provide a valued service to this group who already has hearing aids and needs additional service.

Help seeking individuals who have already purchased hearing devices elsewhere could need follow-up care that can be placed into one of two categories: device mastery skills and self-management skills. Each category of service requires the audiologist (or a trained non-audiology assistant) to customize the fitting, counseling or educational support of the person in need of help. Let us examine these two categories of service, mindful that each can be delivered unbundled from the sale of hearing aids. 


\subsection{Device mastery skills}

Any service delivered by an audiologists that depends on the patient's interaction with their hearing devices can be placed in the device mastery skills category, including:

- Customization of device performance using real ear measures to ensure a prescriptive target is being matched

- Insert and removal of hearing aids from ears

- Basic orientation-how to use features and accessories of devices

- Care and maintenance of devices

- Expectations of initial use of devices

- Pairing device to mobile device and adjustment of SFHAs with app

- Auditory training exercises that include use of hearing devices during the training

Note that many of the components of device mastery are addressed in the user manual of the hearing device. In addition, many of these device mastery skills can be taught via YouTube videos or a smartphone-enabled app.

Once a device mastery plan has been customized for the individual (and when permitted by state regulations) audiologists should consider the use of a welltrained, competent non-audiology assistant to deliver all or part of the patient support of these device mastery skills.

As we peer into the future, SFHAs are likely to become easier to use for a larger segment of the population. As SFHA technology becomes easier to use and meshes seamlessly with smartphones and Bluetooth-enabled devices, it is also likely that the user instruction manual will become more interactive. It is safe to assume that many of the device mastery skills listed above could be replaced by smartphone-enabled apps that help a patient troubleshoot problems associated with their hearing devices. Thus, audiologists should be poised to provide device mastery services to individuals that require face-to-face intervention, perhaps scheduled across several service appointments.

\subsection{Self-management skills}

Hearing loss self-management skills refer to the knowledge and skills people use to manage - as independently as possible - the effects of hearing loss on all aspects of their lives. Moving beyond device mastery skills, teaching individuals to actively identify challenges and solve problems associated with their hearing loss describes the term self-management. For audiologists, providing self-management skills training could be an opportunity to offer a tangible service that stands apart from the delivery of a device.

Given the movement toward more over-the-counter purchases of hybrid hearing devices, it is imperative that audiologists have some tangible services, valued by the marketplace, that fall under the rubric of hearing loss self-management skills. Beyond successfully using hearing aids, hearing loss self-management skills 
encompass maintaining physical and emotional well-being, active monitoring of changes in hearing loss or hearing device effectiveness, and taking an active role in long-term care and decision making. In a paradigm that focuses on improving selfmanagement skills, it is the responsibility of the audiologist to help patients acquire these skills.

\section{Helping patients become better self-managers of their condition}

Self-management skills for adults with hearing loss is defined as the patient independently demonstrating the following behaviors: (1) Active participation in the goal setting and treatment planning process, (2) Adherence to an agreed upon treatment plan, (3) Ability to recognize and manage changes in condition or treatment plan, and (4) Use of proactive coping strategies when communication becomes challenging or treatment plan falls short of expectations. When audiological rehabilitative is viewed through the lens of improving hearing loss self-management skills, the provision of a hearing device from the audiologist is not necessarily needed.

When audiologists improve the self-management skills of adults with hearing loss, several benefits are likely to occur: Individuals, who can effectively selfmanage their condition, are less likely to show up unannounced in the clinic looking for additional help, they are more likely to keep their scheduled appointments and to experience improved outcomes. All of which help a practice operate more efficiently.

It is likely that many adults with hearing loss, regardless of where they purchased hearing devices, will benefit from becoming better self-managers of their condition. If a primary role of audiology is to guide patients through the process of becoming better self-managers, the necessary services provided by the audiologists can probably be placed into one of these three categories: (1) Information gathering and exploratory dialog, (2) Goal setting and treatment planning, and (3) Monitoring progress and assessing outcomes. The foundational skills needed to perform that services are motivational interviewing, shared decision making, and other types of skills directly related to communication and counseling. It is a positive development, for example, to see collaborations between audiology and psychology that are encouraging the use of these skills.

To customize a hearing loss self-management plan for these individuals, Convery et al. [4] developed a self-management interview process centered around assessing the patient's knowledge of their condition and treatment options, actions that can be taken to improve or cope with their condition and coping strategies for difficult communication challenges. In their iteration of a self-management in-take process, they asked patients and audiologist to work together to complete a self-management interview. In this process, the audiologist asks the following questions to the patient:

1. Overall, what do you know about your hearing loss?

2. In general, what do you know about your treatment/management options?

3. How likely are you to manage my hearing loss as asked by your hearing care provider?

4. How likely are you to attend appointments as asked by your hearing care provider? 
5. How likely are you to keep track of changes in your condition (e.g., sudden change in hearing, pain, hearing aids stop working)?

6. How likely are you to work with your hearing care provider to get the services you need?

7. How do you manage the effect of your hearing loss on how you feel (e.g., emotions, well-being)?

8. How do you manage the effects of your hearing loss on your social life (e.g., participate in activities, mix with other people)?

9. How confident are you that you can self-manage your condition effectively?

In addition to asking for responses from patients, the audiologist rated on a 1-8 scale their professional judgment as to patient's ability to complete these tasks. The purpose of the rating and the interview was to individualize the self-management plan. Clinicians should be cautious about utilizing this interview format, as research is still being gathered on how it might apply to persons with hearing loss, especially those who have attempted to self-direct their care. However, responses to these interview questions could form the basis for a customized self-management treatment plan used with anyone in need of help, regardless of where they purchased their devices or what type of devices they are using.

The patient's responses to the interview can be used to create a plan with the goal of assisting the patient become an independent self-manager of his condition. The plan can focus on improving one of the three components of self-management: (1) Knowledge of condition and treatment options, (2) Actions that improve the patient's condition, and (3) Psychosocial issues resulting from the hearing loss that need to be overcome or addressed.

\section{The self-management plan}

The results of the self-management interview can be used to create a customized self-management plan for the patient. The objective of the self-management plan is to guide the patient toward becoming an independent communicator. Figure 5 shows one example of a self-management plan for one older adult.
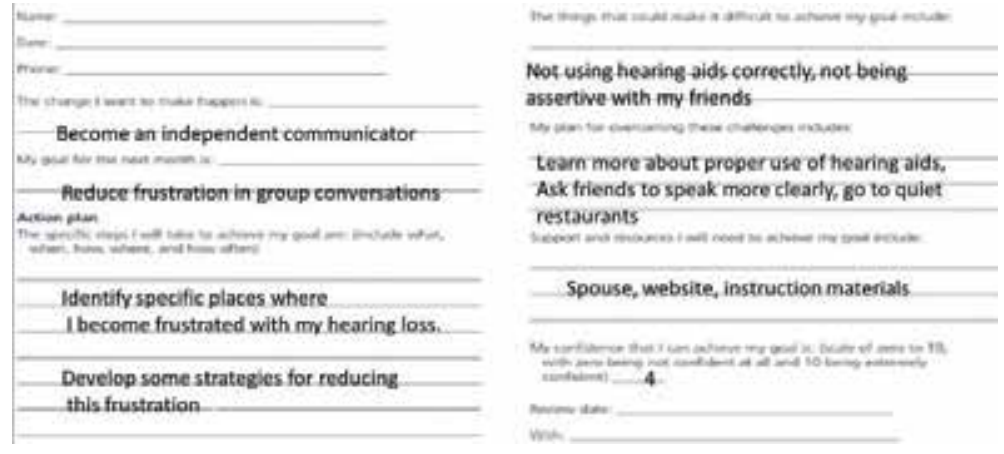

Figure 7 .

An example of a customized self-management plan, which is an iterative process. 
The self-management plan, depicted in Figure 7, is an iterative process, which means that it is likely to change over time. Therefore, at least once a year the audiologist and patient sit down together and update the plan by modified goals and communication strategies. The main point is that a major focus is on the individual with hearing loss and not the mastery of devices, thus any patient who purchased their devices elsewhere are still a prime candidates to benefit from the provision of self-management skills training from an audiologist.

\section{More on hearing aid follow up care}

As experienced audiologists know, a substantial number of patients struggle with "getting used to hearing aids." The term, "getting used to hearing aids" can mean many different things, but for our purposes, it refers to a patient's ability to become a successful hearing aid user, which typically entails a bit of a learning curve as patients learn how to both use the hearing aids and listen to new sounds for the first in several years. If we all agree that successful use is involves full time hearing aid use, as well as some combination of good satisfaction and benefit in real-world listening situations, it is incumbent upon audiologists to help patients get the most from their purchase of hearing aids.

Dawes et al. [5] examined some of the factors associated with getting used to hearing aids. According to their work, there are seven factors that moderate a patient's ability to get used to their devices, which include:

- Acceptance of hearing loss

- Consistent use of hearing aids

- Gradually build up to full time use

- Determination to become a successful user

- Encouragement from others

- Good relationship with the audiologist

- Provision of information about how hearing aids works and the self-management process

Clinical audiologists can use this list of moderators to build a treatment plan that result in a patients becoming a successful wearer. By evaluating each of the seven components and devising a plan to improve shortcomings, the audiologist is directly contributing to an outcome that is more likely to be successful.

\subsection{Customizing a “Getting Used to It” treatment plan}

For each of the seven factors that moderate successful hearing aid use listed above, the audiologist can customize a plan. This process starts by asking one or two questions about each of these factors and devising a strategy for improving it. For example, for the factor of determination to be a successful user, the audiologist could ask some frank questions about exactly how determined a patient is to do what it takes to be successful. For those lacking determination, a strategy could be developed that addresses this gap. 
Nearly every patient fitted with hearing aids in a clinic returns for several hours of additional follow-up care. Spread over the course of four or more years, these face-to-face visits with the clinician gobble up a lot of precious clinical time, but the additional time does not always result in favorable patient outcomes. For example, Bennett et al. [6] suggest the needs of adult hearing aid owners are not being adequately addressed during their follow-up appointments with an audiologist. According to their work, $90 \%$ of hearing aid owners demonstrated difficulty with basic hearing aid management tasks, such as inserting the device into the ear or properly cleaning it. They also reported that almost one-half of hearing aid owners did not receive enough practical help about their hearing aid use. Obviously, insufficient training and support can lead to poor outcomes and non-use of hearing aids. But just how widespread of a problem this poses is a question that warrants further analysis.

Rebecca Bennett of the Ear Sciences Centre at The University of Western Australia and her colleagues have addressed these apparent gaps in the informational and training needs of adults fitted with hearing aids. Using a research method called concept mapping, an approach previously used to study help seeking behaviors in adults with hearing loss, Bennett and her colleagues evaluated the opinions of both hearing aid owners and clinicians about their knowledge, skills and tasks required to use, handle, care and maintain hearing aids. Described by the researchers as hearing aid self-management skills, the main objective of their work is to better understand the key skills and attributes of adult hearing aid users, so that clinicians can deliver a better quality of care to individuals after they have been fitted with hearing aids.

In studies published in the peer reviewed journals, American Journal of Audiology and Ear and Hearing, Bennett and her colleagues identified more than 100 unique descriptors of the hearing aid management process that could be broken down into 6 separate concepts that influenced hearing aid use and quality of follow-up care: (1) working with your clinician, (2) communication strategies, (3) learning to come to terms with hearing aids, (4) hearing aid maintenance and repairs, (5) daily hearing aid use, and (6) advanced hearing aid knowledge.

Items 1-3 were classified as person-centered attributes, while items 4-6 were classified by the researchers are device-centered attributes. Hearing aid owners (24 of them participated in the study) indicated that all six concepts were similarly important, whereas clinicians (22 participated in the study) indicated that advanced hearing aid knowledge was less important to long-term success of the patient than the other five concepts.

Despite the on-going support offered to clients after they acquire hearing aids, they are often hesitant to seek help from their clinician, and instead engage in a myriad of helpful and unhelpful behaviors in response to problems that arise with their hearing aids. Previous positive and negative experiences with the clinic, clinician and significant other influenced these actions, highlighting the influential role of these individuals' in the success of the rehabilitation process. This data suggests that clinicians could improve hearing aid problem resolution by providing technical and emotional support, including to significant others by promoting client empowerment and self-management.

\section{The importance of a therapeutic relationship during follow-up care}

Hearing aid wearers who participated in the study noted the importance of an effective working relationship between hearing aid wearer and audiologist. Traits of a good therapeutic relationship with their audiologist, such as awareness, 
understanding, knowledge and a willingness to help were valued by hearing aid owners. On the other hand, patient traits, like proactive, help seeking behavior, knowing when to ask questions to the clinician, being comfortable divulging personal information, and asking for help contributed to a strong working relationship between patient and clinician.

The work of Bennett and colleagues cited previously serves as a reminder that successful long-term hearing aid use by patients has two distinct components: mastery of the device and independent problem-solving, self-management skills. To teach patients about these two components require audiologists possess both effective technical skills and interpersonal counseling skills. A careful reading of Bennett's work suggests clinicians need to excel at both: many clinicians focus too much of their attention on the technical aspects of the device at the expense of building a strong therapeutic relationship with the person.

Finally, it should be apparent that a clinician's role is much more than providing verbal instructions on how to handle and maintain hearing aids-one of the main topics covered during hearing aid follow-up appointments. It is equally important to establish whether patients have learned skills that allow for mastery of their device and self-managed problem-solving skills. Clinicians who are proficient at teaching patients both skills, especially in a changing market where patients might purchase hearing aids on-line, and then seek professional guidance, offer a professional service that cannot be duplicated by lower skilled technicians or machine learning algorithms.

Using Bennett et al.s [6] work as a foundation, here are five tasks audiologists can do during routine follow-up appointments to ensure patients are getting the most from their hearing aids over the next several years:

1. Empowerment. Help patients recognize and independently solve communication problems. The process of empowerment can be facilitated by getting patients involved in decision making and supporting their treatment choices. The use of easy-to-understand, visually appealing decision aids that present patients with a range of treatment options and tips for independently solving common communication breakdowns can be used to help patient's feel empowered. We know, see https://www.ncbi.nlm.nih.gov/pubmed/21841487, for one example, that individuals who are given a range of treatment and hearing loss management choices are more likely to actively participate in the rehabilitation process.

2. Avoid Information dumping. Convey technical information in ways that are easy for patients to understand. Provide them with concise printed materials that they can refer to after the appointment. Be sure that the instructional materials are easy to read, use pictures to reinforce key points and are branded to your clinic.

3. Considering breaking appointments into smaller chunks. To ensure patients understand all aspects of successful hearing aid use, consider bringing the patient back more often for follow-up appointments, or better yet, use Skype and other forms of video conferencing to relay information to the patient in smaller chunks. Utilize support personnel whenever possible in the follow-up care and support process to ensure your clinic operates efficiently. Allowing an audiology assistant to participate in the follow-up process is an effective way to break the monotony some patients experience when trying to learn from one instructor over a lengthy period. By getting an assistant involved in teaching patients some of the routine aspects of follow-up care, it frees the audiologist's schedule to see new patients. 
4. Break the hearing aid check and other similar follow-up appointments into "knowing how" and "knowing when" buckets. "Knowing how" refers to physical, hands-on skills patients must acquire to be successful hearing aid users. "Knowing when" skills are more abstract and require clinicians teach patients more complex tasks that require higher level cognitive awareness and skill, such as knowing when to use a remote microphone, knowing when to recognize a challenging listening situation that requires some modification of listening behavior, or knowing when know how to be a more assertive, proactive listener. Tailor instructional materials to help patients identify when they need to modify a behavior to be a more effective communicator.

5. Encourage patients to keep a diary of their initial listening experiences. This enables patients to keep more directly involved in their follow-up. Ask patients to spend a couple minutes at the end of the day to reflect on their listening experiences, how they feel about each of them and what they did in reaction to their feelings. By keeping a diary for the first month or so of hearing aid use facilitates activity involvement on the part of the patient as a problem solver and fosters their ability to be an independent communicator.

Beyond adjusting the acoustic parameters of hearing aids and assisting patients with the hands-on skills needed to use their hearing aids, there are an abundance of person-centered skills that are too often overlooked by clinicians, but desired by patients. The work of Bennett and her colleagues lays the groundwork for how knowledge, skills and tasks can be conveyed to patients in a meaningful way, thus enabling them to be independent, self-managers of their communication.

While clinical audiologists to shed light on new approaches to care, there are some things we can do to add value to the follow-up process.

In summary, the following types of services could be offered to help seekers who already own hearing devices:

- Diagnostic audiological assessment to identify possible underlying medical complication that requires a physician referral

- A quality control check of their devices to ensure they are meeting a validated standard, either in the ear with probe microphone measures or in the coupler with a hearing aid test box.

- Basic communication assessment to identify extent of problem followed by one of the following services:

- Device customization and/or device mastery training, possibly delivered by a non-audiologist

$\circ$ Hearing loss self-management skills training

- Customized treatment plan that focuses on "getting used to hearing aids"

\section{Beneficiaries of self-directed care}

Considering the low update of hearing aids, the advent of self-directed care and self-fitting hearing aids has the potential to expand the market for services. Below are four underserved segments of the hearing care market that could benefit from 
systematic follow-up care that is decoupled from the sale of devices. Audiologist can expect a growing number of these individuals coming to their clinic for guidance, support or service after hearing aids have been purchased.

\section{Four underserved patient categories}

There are at least four types of individuals, currently underserved by hearing aids that are candidates for these alternative devices. Three of the four categories are commonly encountered in the clinic, while the fourth category are in great abundance, but rarely find their way to a clinic for reasons we will discuss later. Table 1 is a summary of these four underserved patient categories. Here are some added details on each one.

1. The Older Old. Prone to cognitive decline and physical limitations, this group, which also tends to be socially isolated, is susceptible to losing their hearing aids. Additionally, they are often unlikely have the physical or cognitive capacity to wear hearing aids consistently. Thus, many in this group become non-hearing aid users. Neckband multi-tasker PSAPs or other devices for situational use may be a viable alternative for this group.

2. The Contemplator. The Stages of Change model, which describes the behaviors and attitudes of people with chronic conditions over a period of time, suggests that individuals in the early contemplation stages are still working though the burdens their hearing loss places on daily life. Thus, they are not ready to act with respect to addressing their condition. Allowing a patient to dabble in a low-risk way from the comforts of home, has the potential to enable the contemplator to act sooner, on their own terms. In this scenario, the initial use of a non-custom product is a gateway product that culminates in the future with full time hearing aid use.

3. The Patient with Cochlear Distortion. The cochlea, for a small number of individuals, fails to carry information to higher regions of the auditory system. Researchers have surmised that these cases can be identified by measuring word recognition at a low intensity level and comparing the results at a higher intensity. (A poor result on the Quick SIN also might be an indicator of this condition.) Unlike the typical performance-intensity function that shows improvement in word recognition ability as audibility of speech is increased, patients with cochlear distortion issues fail to show improvement in word recognition ability. Because the patient with cochlear distortion fails to experience the same improvement in speech understanding when audibility is restored with hearing aids, it is presumed non-custom devices might be a more costeffective choice for these cases, as a fully featured set of hearing aids could be considered technological overkill.

The three previously mentioned groups of patients are likely to seek the services of an audiologist for testing or guidance. The final category, because they often have normal or near-normal hearing aid and do not consider their hearing to be a "problem," are unlikely to seek help from an audiologist. Therefore, this group must be reached in other ways.

Tech Savvy Overshoots: There are many adults, often between the ages of 50 and 65 , that experience occasional difficulty with their hearing, but do not think they have a problem that warrants a visit with an audiologist. Because they are younger, 


\begin{tabular}{|c|c|c|c|}
\hline Group & Red flag characteristics & How to identify & $\begin{array}{l}\text { Non-custom solution } \\
\text { to consider }\end{array}$ \\
\hline Older old & Chronological age $>85$ & $\begin{array}{l}\text { Poor scores on cognitive } \\
\text { and haptic screen }\end{array}$ & $\begin{array}{l}\text { Ear-level neckband } \\
\text { PSAP, ALD or } \\
\text { non-custom headset } \\
\text { amplifier }\end{array}$ \\
\hline Contemplator & $\begin{array}{l}\text { During interview blame } \\
\text { other people or the } \\
\text { environment for their } \\
\text { hearing problem }\end{array}$ & During interview process & $\begin{array}{l}\text { Ear-level PSAP, ALD, } \\
\text { or smartphone- } \\
\text { enabled app + wired } \\
\text { earbuds }\end{array}$ \\
\hline $\begin{array}{l}\text { Cochlear } \\
\text { distortion }\end{array}$ & $\begin{array}{l}\text { Poor word recognition } \\
\text { score at } \mathrm{PB} \text { max }\end{array}$ & $\begin{array}{l}\text { No change in word } \\
\text { recognition between } 45 \\
\text { and } 75 \mathrm{~dB} \text { presentation } \\
\text { levels, poor QSiN score }\end{array}$ & $\begin{array}{l}\text { Ear-level wireless, } \\
\text { neck-band PSAP or } \\
\text { traditional hearing aid }\end{array}$ \\
\hline $\begin{array}{l}\text { Tech savvy } \\
\text { middle agers }\end{array}$ & $\begin{array}{l}\text { Struggle in with their } \\
\text { hearing in one or two } \\
\text { challenging listening } \\
\text { situations }\end{array}$ & $\begin{array}{l}\text { Self-assessment tools on } \\
\text { a clinic-branded website }\end{array}$ & $\begin{array}{l}\text { Ear-level wireless } \\
\text { or neck-band PSAP } \\
\text { with multi-tasking } \\
\text { capability }\end{array}$ \\
\hline
\end{tabular}

Table 1.

A summary of the four groups, possibly under-served in today's marketplace, who may be receptive to the use of non-custom devices.

tech savvy individuals they might be open to a do-it-yourself approach to finding help in situations where hearing is a challenge. In the past, if these individuals were to find their way to an audiology clinic, they were offered a $\$ 3000$ solution for a problem they perceive to be worth fixing for less than $\$ 500$. By combining amplification with other features that they find useful in their busy lives, the middle aged, tech savvy individual could address their communication challenges with any number of high quality ear-level PSAPs. Because many of these individuals will not seek the services of the audiologist in a clinic, we can use tools like the internet or a well-designed website to reach them. Although traditional hearing aids would be appropriate choices from a prescriptive fitting target standpoint, the style or function of traditional hearing aids may negate the trial and use of amplification as a treatment option.

Considering less than $30 \%$ of adults with hearing loss use hearing aids, a primary challenge for a medical clinic is attracting individuals that need help with their hearing into your practice. One approach to broadening the market for audiology is to recommend high quality non-custom amplifiers to adults who are not viable candidates for traditional hearing aids. Although traditional hearing aids are likely to remain the gold standard for adults with benign cases of hearing loss, clinicians should embrace vetted non-custom amplifiers as a solution for the appropriate candidate.

\section{Overcoming the tyranny of free tests and unit margins}

Ultimately, the onus of addressing the unmet needs of those with hearing loss falls to the profession. It is incumbent upon all of us to find innovative approaches to service device provision that get more individuals coping with the ill-effects of untreated hearing loss involved in the process of improving their own hearing and communication. This, after all, is the essence of the chronic care model: To help these patients become better, more effective self-managers of their own condition. 
Audiology's Third Pillar: Comprehensive Follow-Up Care and Counseling for Those Who Choose... DOI: http://dx.doi.org/10.5772/intechopen.88224

\begin{tabular}{|c|c|c|}
\hline & $\begin{array}{l}\text { Help seekers without hearing } \\
\text { devices }\end{array}$ & Help seekers with hearing devices \\
\hline $\begin{array}{l}\text { Information gathering } \\
\text { and exploratory dialog }\end{array}$ & $\begin{array}{l}\text { 1. Assess "importance to treat" (low } \\
\text { or high) } \\
\text { 2. Determine potential to self-fit their } \\
\text { own hearing aids } \\
\text { 3. Collect audiological and non- } \\
\text { audiological information about } \\
\text { patient following ICF model }\end{array}$ & $\begin{array}{l}\text { 1. Gather objective information on } \\
\text { current hearing devices } \\
\text { 2. Collect audiological and non- } \\
\text { audiological information about patient } \\
\text { following ICF model } \\
\text { 3. Conduct objective assessment of } \\
\text { current hearing devices, using PMM }\end{array}$ \\
\hline $\begin{array}{l}\text { Goal setting and } \\
\text { treatment planning }\end{array}$ & $\begin{array}{l}\text { 1. Target areas of improvement } \\
\text { 2. Option talk } \\
\text { 3. Choice talk } \\
\text { 4. Align goals and expectations with } \\
\text { treatment options (pre-treatment) }\end{array}$ & $\begin{array}{l}\text { 1. Conduct self-management } \\
\text { interview } \\
\text { 2. Target areas of improvement } \\
\text { 3. Align goals and expectations with } \\
\text { current treatment (or recommend } \\
\text { new treatment plan) }\end{array}$ \\
\hline $\begin{array}{l}\text { Assessing outcomes } \\
\text { and monitoring } \\
\text { progress }\end{array}$ & $\begin{array}{l}\text { 1. Align goals and expectations with } \\
\text { treatment option (post-treatment) } \\
\text { 2. Look for areas of improvement: } \\
\text { device mastery and/or self- } \\
\text { management skills } \\
\text { 3. One-year post fitting: conduct } \\
\text { self-management interview }\end{array}$ & $\begin{array}{l}\text { 1. Align goals and expectations with } \\
\text { treatment option (post-treatment) } \\
\text { 2. Look for areas of improvement: } \\
\text { device mastery and/or self- } \\
\text { management skills }\end{array}$ \\
\hline
\end{tabular}

$I C F=$ international classification of functioning, $P M M=$ probe microphone measures.

Table 2.

A summary of various clinical procedures that could be offered to two different types of patients that audiologists can expect to see once OTC and self-fitting products are widely available.

For decades, it was sustainable business practice to provide free hearing tests and dispense, on average, 15-20 hearing aids per month to be profitable. Even if you provide the very best patient care, the units based business model is unlikely to be sustainable over the long haul in a profession that will see shrinking margins resulting from the availability of OTC device, third-party insurance contracts and other innovations that appeal to persons with hearing loss.

By focusing on the emotional, psychosocial and functional impact that hearing loss has on the person's ability to self-manage their condition, audiologists can provide a full range of counseling and customization services-beyond the traditional bundled approach to delivering audiologist-driven care. These new services could be appealing to a broader range of persons with hearing loss who choose to self-direct their care and could complement current clinical practice.

A primary focus of this chapter was to provide some practical insight on how self-fitting hearing aids and other amplification devices purchased over the counter (OTC), might change the way patients interact or connect with audiologists in their clinic. Although no one can predict the future, it is safe to say the availability of self-fitting hearing aids as well as other OTC devices that allow people to self-direct their care will have an impact. It is likely individuals who have already purchased a hearing device over the counter will seek the services of an audiologist. Thus, audiologists must be prepared to offer them a service of value.

To summarize, three different clinical tasks used during the Communication Assessment are outlined in Table 2: information gathering \& exploratory dialog, goal setting and treatment planning, and assessing outcomes and monitoring progress. Along with the three clinical tasks, Table 2 summarizes the key work of audiologist for those three dimensions of care for two types of help seekers. As we move into a future sure to be filled with self-fitting hearing aids, automated hearing testing and other consumer-driven healthcare initiatives, audiologists will serve 
as advisors and consultants. When patients have questions or concerns, no matter where they purchased their hearing devices (or if they own them at all), they will seek the services of audiologists. Rather than limiting the role of audiology to selecting, fitting and tweaking hearing aids, Table 2 demonstrates that in the emerging era of self-directed care, the potential value of audiology is evaluating the entire person and offering solutions, many of which are not device-related, that help patients become better, more effective self-managers of their condition.

Audiologists must anticipate a future filled with several options that allow patients to self-direct their care. From the point of view the massive numbers of people with untreated hearing loss, the provision of new direct to consumer choices is a positive development. Rather than scoff at this change, audiologist would be wise to embrace it and identify ways they can add value for those who opt to selfdirect their care and then find they need some additional support or guidance from an expert. The objective of this chapter was to spur thinking on novel approaches to service and review some of these approaches that can be implemented in a clinic today. Now is the time for audiologists to create the future-a future less dependent on the sale of a device.

\section{Author details}

Brian Taylor

WS Audiology and A.T. Still University Golden Valley, Minnesota, United States

*Address all correspondence to: brian.taylor.aud@gmail.com

IntechOpen

(C) 2019 The Author(s). Licensee IntechOpen. This chapter is distributed under the terms of the Creative Commons Attribution License (http://creativecommons.org/licenses/ by/3.0), which permits unrestricted use, distribution, and reproduction in any medium, provided the original work is properly cited. (cc) BY 
Audiology's Third Pillar: Comprehensive Follow-Up Care and Counseling for Those Who Choose... DOI: http://dx.doi.org/10.5772/intechopen.88224

\section{References}

[1] Abrams HB, Kihm J. An introduction to MarkeTrak IX: A new baseline for the hearing aid market. Hearing Review. 2015;22(6):16

[2] Humes LE, Rogers SE, Quigley TM, Main AK, Kinney DL, Herring C. The effects of service-delivery model and purchase price on hearing-aid outcomes in older adults: A randomized doubleblind placebo-controlled clinical trial. American Journal of Audiology. 2017;26(1):53-79

[3] Keidser G, Convery E. Outcomes with a self-fitting hearing aid. Trends in Hearing. Jan-Dec 2018;22. Published online; 1 May 2018

[4] Convery E, Hickson L, Meyer C, Keidser G. Predictors of hearing loss self-management in older adults. Disability and Rehabilitation. Aug 2019;41(17):2026-2035

[5] Dawes P, Maslin M, Munro K. 'Getting used to' hearing aids from the perspective of adult hearing aid users. International Journal of Audiology. 2014;53:861-870

[6] Bennett RJ, Meyer CJ, Eikelboom RH. How do hearing aid owners acquire hearing aid management skills? Journal of the American Academy of Audiology. 2019;30(6):516-532 



\title{
Progenitor Cell Therapy for Sensorineural Hearing Loss in Infants
}

\author{
Linda Baumgartner, Michael Seidman, Deborah Lamontagne, \\ Ernest Moore, David Shook, Steven Messina \\ and James Baumgartner
}

\begin{abstract}
Typical language development requires typical hearing. With sensorineural hearing loss (SNHL), the damaged hair cells of the organ of Corti within the cochlea interfere with typical hearing and, as a result, cause impaired language development. Untreated SNHL causes significant neurocognitive differences in affected children. SNHL is a permanent sensory disorder affecting more than 270 million people worldwide. Congenital SNHL is found in 4 of 1000 newborns. Approximately half of congenital SNHL is hereditary and is the result of genetic mutations causing improper development of cochlear hair cells. Non-genetic congenital SNHL is thought to be the result of an injury to the cochlea typically from premature birth, infection, or exposure to ototoxic medications or noise. In mammals, the cochlea is postmitotic at birth, and no spontaneous repair occurs thereafter. Existing treatments for SNHL (hearing aids and cochlear implants) function by augmenting the damaged organ of Corti. No reparative treatments currently exist. In preclinical and clinical studies, progenitor cell therapy (cord blood and mesenchymal stem cells) has shown promise in reversing the underlying pathology of SNHL, the loss of cochlear sensory hair cells. Progenitor cell therapy may also allow functional reorganization of the auditory pathways including primary auditory cortex (Heschl's gyrus). We will present a summary of the effect of hearing loss on auditory development, existing preclinical and clinical data on progenitor cell therapy, and its potential role in the (re)habilitation of non-genetic SNHL.
\end{abstract}

Keywords: sensorineural hearing loss, human umbilical cord blood, stem cell, progenitor cell therapy, cochlea, auditory verbal therapy

\section{Introduction}

Affecting more than 270 million people worldwide, sensorineural hearing loss (SNHL) is a permanent sensory disorder which interferes with hearing. SNHL is found in 4/1000 in newborns, 8/1000 children aged 3-17 years, and 33\% of adults aged 65-74 years [1-3]. Existing treatments (hearing aids and cochlear implants) improve the symptoms of SNHL by augmenting the damaged organ of Corti. These treatments do not reverse the underlying pathology of SNHL nor loss of sensory inner 
hair cells within the organ of Corti. Inner and outer cochlear hair cells are necessary for hearing and transforming sound waves into electrical impulses transmitted to the brain. Loss of hair cells reduces auditory input to the brain, and with sufficient hair cell loss, hearing impairment develops. In mammals, the organ of Corti is postmitotic at birth, and no spontaneous hair cell regeneration occurs thereafter.

Among infants and children with SNHL, 23-50\% is the result of a genetic mutation that adversely affects development of the organ of Corti (connexin 26, mutation, Waardenburg syndrome, Usher syndrome, Mitochondrial Disorders, etc.) [2-9]. The remaining infants and children have acquired SNHL, which is most commonly attributed to prematurity, infection (in utero or postdelivery), and exposure to noise or ototoxic drugs.

In preclinical and clinical studies, the intravascular delivery of mesenchymal progenitor cells following acute neuro-pathologic insults (stroke, traumatic brain injury, spinal cord injury, etc.) has shown significant promise [10-16]. Limited animal and human data suggest that repair of the mammalian cochlea is possible following progenitor cell therapy $[3,17-20]$. If these early results can be translated to a reparative treatment for SNHL, it would be a transformative advance in auditory (re)habilitation.

\subsection{Hearing loss and auditory development}

Spoken language is learned and its development is dependent upon both the innate ability found within the human cortex as well as environmental stimulation. The time frame over which the cortex is capable of learning a fist spoken language is finite due to neuroplasticity [21]. Neuroplasticity refers to changes in neural connections, pathways, and networks as a result of maturation and development, sensory deprivation, injury, disease, dysfunction, and learning [22]. Although neuroplasticity exists to some degree throughout life, it is particularly robust during early life when neuronal groups are most capable of adjusting function based upon input. This window of heightened learning, known as the critical period, lasts roughly through 3 years 6 months of age. The critical period is a time when the brain effortlessly rewires in response to the environment, and at the end of which there is a decisive diminishing of neuroplasticity.

Auditory development is particularly sensitive to the critical period. Auditory learning begins in utero [23] when synapses are formed and then strengthened at a remarkable rate [24]. At $\sim 4$ years of age, the abundant neurons within the auditory cortex undergo a rapid pruning phase, during which neurons and their synapses are eliminated when unused, and thus considered unnecessary $[25,26]$. This pruning fundamentally alters the auditory cortex, which for the typically hearing child, equals improved language efficiency. Conversely, for the unamplified child with SNHL, pruning results in an inability to develop spoken language. It has been observed that if auditory stimulation is not delivered during the early optimal period of cortical plasticity, deficits are observed even after the child is amplified [27, 28]. A biomarker for auditory cortical maturation is the latency rates of the P1 component of the cortical auditory evoked potentials (CAEP). It has been demonstrated that the $\mathrm{P} 1$ component of the CAEP shows age-related decreases in latency, meaning faster transmission, in children without hearing loss. In a series of 245 children with congenital deafness, Sharma and Dorman found that the latency of the P1 CAEP decreases to within normal limits in children who receive a cochlear implant by 3.5 years of age. Children implanted after the age of 7 years demonstrate abnormal P1 CAEP responses which persisted even after years of experience with implant use. Children implanted between 3.5 and 7 years showed mixed auditory cortical development, with some children demonstrating normal P1 
CAEP responses and others never reaching normal central auditory maturational status [28]. Supporting this finding are studies describing developmental outcomes of speech and language skills in children implanted at various ages, which indicate significantly improved outcomes with younger implantation age [29-31]. Improved outcomes are especially true in the development of oral spoken language [32].

In summary, when a child with SNHL is provided auditory access through hearing aids or a cochlear implant in a timely manner within the critical period, auditory development, and language acquisition may occur normally. Conversely, children who experience long periods of auditory deprivation are susceptible to large-scale reorganization of the auditory cortex areas responsible for the perception of speech and language [33]. When that reorganization happens, there is evidence that several areas of auditory cortex are recruited for visual and tactile input under the condition of auditory deprivation [34-37]. To date, the only task specific reorganization of the auditory cortex that has been proven is in deafened cats. Meredith and Lomber demonstrated that distinct auditory regions in cats with SNHL support peripheral visual localization and visual motion detection, and that the same regions support auditory localization in hearing cats [38].

\subsection{Preclinical evidence for stem cell efficacy in the treatment of SNHL}

Animal studies using mesenchymal progenitor cells have provided intriguing results in experimentally deafened animals. Using NOD-SCID mice experimentally deafened with kanamycin and noise, Revoltella et al. reported recovery of auditory function following intravenous treatment with CD-133+ cells derived from human umbilical cord blood. Some of the cord blood stem cells were shown to have reached the cochlea [17]. In a subsequent study from the same group, Bettini et al. treated NOD-SCID mice deafened with kanamycin with mesenchymal stem cells derived from either bone marrow or adipose tissue. Both cell types engrafted in the cochlea of damaged mice, inducing regeneration of the damaged sensory structures. Several hybrid human-mouse fusion cells were found within the cochlea but not in hair cells. The data suggest that human MSCs do not directly replace lost cells, but exert their regenerative potential mainly through paracrine effects $[17,18,39]$.

Using an SNHL guinea pig model, Choi et al. demonstrated both physiological and anatomic improvement in the cochlea of animals treated with mesenchymal stem cells derived from human umbilical cord blood. Distortion-product otoacoustic emissions (DPOAEs) were decreased and auditory brainstem response (ABR) thresholds were improved by $40-50$ decibels $(\mathrm{dB})$ in treated guinea pigs. In addition, treated animals demonstrated an increase in both hair cells and spiral ganglion cells compared to control animals [19].

\subsection{Clinical evidence for stem cell efficacy in the treatment of SNHL}

DaCosta et al. reported the effect of cord blood transplantation on SNHL following myeloablation in patients with mucopolysaccharidosis [20]. The mucopolysaccharidoses (MPS) are a group of lysosomal storage diseases in which there is a deficiency in one of the enzymes responsible for the breakdown of glycosoaminoglycosides (GAGs). The progressive buildup of GAGs in cells causes tissue and organ injuries. Most patients with MPS present with a mixed hearing loss. As MPS progresses, GAGs accumulate in the tissues of the nasopharynx ultimately interfering with Eustachian tube function and causing chronic otitis media. MPS types 1 and 2 commonly also develop SNHL. The exact etiology of the MPS associated SNHL is not clear but may be a genetic congenital SNHL vs. an acquired injury secondary to the accumulation of GAGs in the cochlea or cochlear nerve [40]. 
The only treatment that demonstrates long-term metabolic correction and neurocognitive improvement in MPS is hematopoietic stem cell transplantation [41, 42]. In DaCosta's series, 26 of 30 patients had MPS 1 and 2. Following bone marrow transplantation, the ABR click threshold improved by $19 \mathrm{~dB}$ on average and 20 of 30 patients experienced an improvement in sensorineural hearing. The effect on SNHL was more prominent in children who underwent bone marrow transplantation at less than 25 months of age. The cord blood used for transplantation was allogenic and did not carry any of the MPS mutations [20].

\subsection{Phase 1 trial: umbilical cord blood therapy for acquired SNHL in children}

In a Phase 1 trial, 11 children less than 6 years of age, with severe to profound non-genetic SNHL were treated with their own umbilical cord blood mononuclear fraction intravenously. Subjects were recruited from a single private cord blood bank, cord blood registry, through the bank's patient email portal. Patients were evaluated before treatment and 1-, 6-, and 12-months posttreatment. Evaluations included physical and neurological examinations, speech language pathology testing, audiology evaluations, 3-Tesla MRI with diffusion tensor imaging (DTI), and laboratory testing.

No significant adverse events occurred during the study. Ten subjects experienced an expected improvement of speech language pathology test scores over the course of the trial. The only subject who failed to improve did not follow study mandated amplification and recommended auditory verbal speech language therapy, demonstrating the importance of speech language therapy in this vulnerable population. About 5 of the 11 treated subjects experienced an improvement in ABR thresholds which achieved statistical significance across the treatment population at three measured frequencies. The improvement in ABR threshold ranged from 15 to $20 \mathrm{~dB}$ (Figure 1). There was a trend toward improvement in the latency of signal transmission along cranial nerve VIII (Vestibulo-cochlear nerve). Improvements in both ABR thresholds and CN VIII latency were evident at 1-month follow-up testing and were durable throughout the 12-month study period. The rapid and durable change in latency was unexpected.

Using 3 T MRI data collected before and 12-months after cord blood treatment, subjects whose ABR thresholds had improved following were compared to subjects whose ABR thresholds had not changed following treatment (Figure 2). The DTI measure fractional anisotropy (FA), a marker of white matter tract integrity and myelination [43], trended toward improvement along the auditory pathways in responding subjects. The changes in FA were most prominent in the white matter of Heschl's gyrus, which is the primary auditory cortex (Figure 3).

All responding subjects received a cord blood cell dose of at least 15 million cells per kilogram [3].

This trial supports the concept that autologous intravenous cord blood therapy can facilitate repair of the cochlea. The data also suggest that improvement in the entire auditory pathway might occur following the progenitor cell therapy.

\subsection{Possible mechanisms of action}

While limited, the existing data suggest that progenitor cell therapy does not result in direct replacement of cochlear hair cells, but enables an intrinsic repair machinery to work. The immunomodulatory effect of MSCs acting systemically and the local effect of MSCs which reach and interact with the cochlear stroma and possibly cochlear stem cells may facilitate hair cell replacement. A similar process 

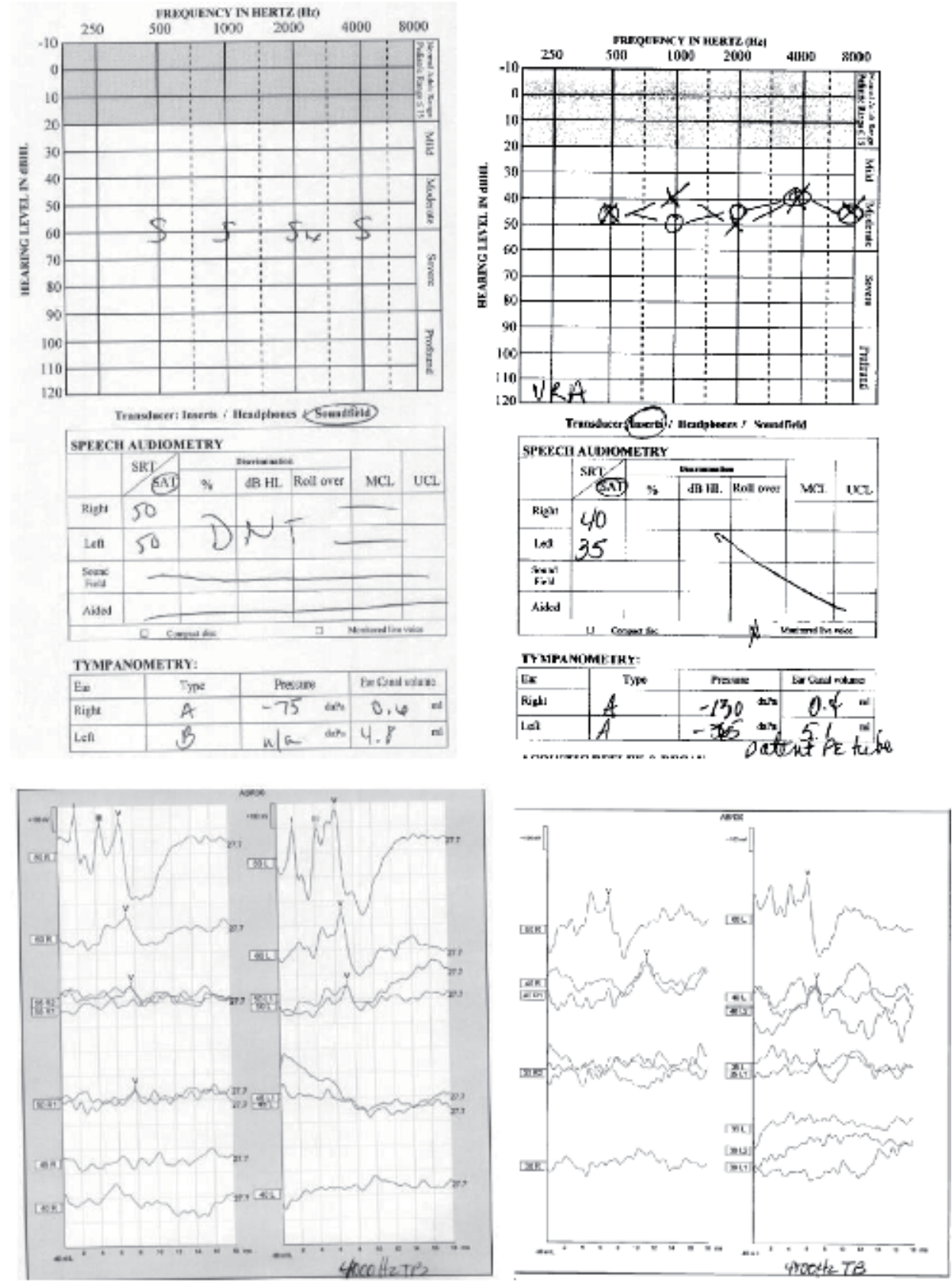

Figure 1.

Representative audiograms (top) and $A B R$ recordings at $4000 \mathrm{~Hz}$ (below) of a responding subject before (left) and after (right) hUCB treatment for SNHL. The improvements on the behavioral testing (audiogram) match the changes found on the ABR recordings (physiologic).

involving MSCs which cross the blood brain and blood labrynthine barriers and interact directly with brain tissue may facilitate repair and reorganization of the white matter tracts of the auditory pathway.

While some infused mesenchymal progenitor cells do cross the blood brain barrier and reach the cochlea $[17,18]$, the majority fail to do so. Because mesenchymal stem cells have cell diameters larger than most terminal arterioles, most infused MSCs are found within the capillaries of the lungs within minutes of infusion 


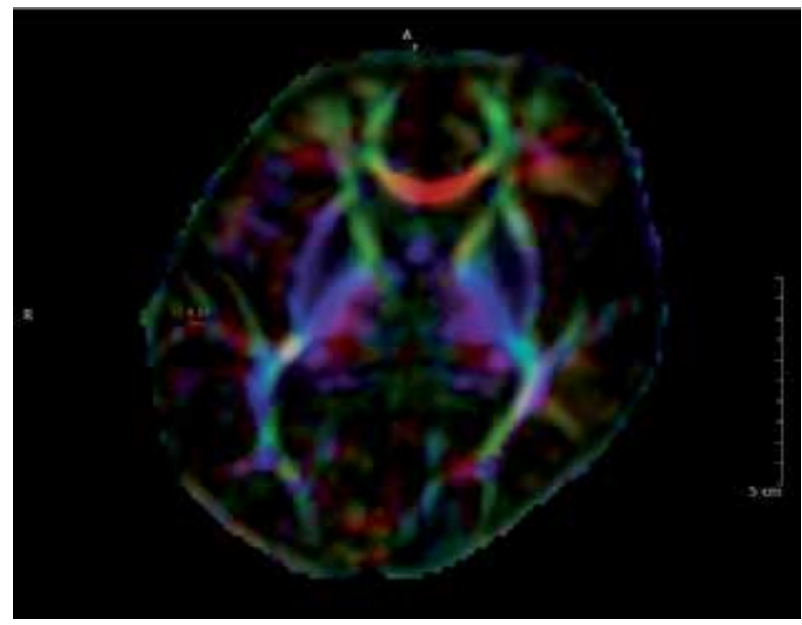

Figure 2.

Raw axial DTI image with the ROI of the right sided Heschl's gyrus used for FA analysis, outlined in red.

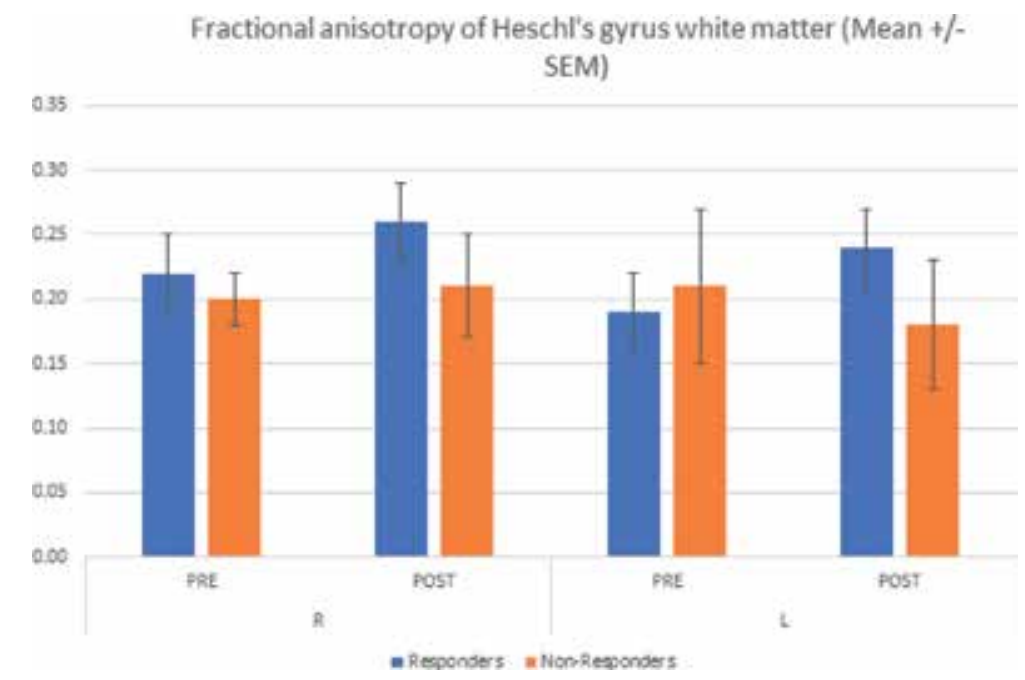

Figure 3.

Graphical representation of mean fractional anisotropy between responding (blue) and non-responding (orange) subjects at region of interest sites in Heschl's gyrus following cord blood mononuclear treatment for SHNL in children. The data suggest an increase in fractional anisotropy in responding subjects, but not in nonresponders. An increase in the fractional anisotropy suggests improved white matter tract integrity and possibly the repair of primary auditory cortex.

[44-46]. In both humans and animals, this rapid pulmonary entrapment is followed by clearance from the lungs and accumulation in the liver and spleen over subsequent hours to days [43-45]. The MSCs, while entrapped, cause a marked change in circulating cytokines and immune system phenotype [47]. Notably, human MSCs have been shown to be capable of migrating to an area of injury and recruiting tissue specific progenitor cells and regulating the immune response through the secretion of immunomodulatory cytokines and microvesicles (exosomes) containing a variety of bioactive molecules including enzymes, coding and non-coding RNAs, and growth factors [48]. MSCs are also known to secrete molecules that modulate both innate and adaptive immune responses [49]. These secreted molecules act to inhibit the maturation of monocytes into antigen presenting dendritic cells [50], promote a shift in macrophage phenotype from M1 to M2 [51], inhibit the 
proliferation and activation of B and T lymphocytes [52], and promote the clonal expansion of regulatory T lymphocytes [53]. This extensive systemic alteration of the immune system may facilitate repair through a systemic paracrine effect.

Likewise, the previously identified fusion of MSCs with cochlear support cells may also allow cochlear support or cochlear stem cells to differentiate into hair cells [18]. Epigenetic regulation of regeneration has recently emerged as a possible pathway to hair cell replacement [54]. The improved FA found at Heschl's gyrus may represent a rescue of the auditory cortex from sound deprived visual fate back to its original hearing function. That recovery appears to depend upon repair of the cochlea, the spiral ganglion, the eighth cranial nerve, and the white matter tracts of the auditory pathways. All of these repairs may be facilitated by intravenous mesenchymal stem cell treatment [55].

\section{Conclusion(s)}

SNHL is a permanent sensory disorder and a significant worldwide public health problem. Untreated sound deprivation causes permanent reorganization of the auditory pathways that first interferes with and then prevents the development of spoken language. Current treatments augment the function of a damaged cochlea and no reparative treatments currently exist. Both preclinical and clinical data suggest that treatment with progenitor cells may result in cochlear repair in mammals. In addition, very limited data suggest that the repair process may extend beyond the cochlea to the auditory pathways and auditory cortex. This evolving area of research may allow the development of a reparative treatment for non-genetic SNHL.

\section{Acknowledgements}

The cord blood phase one trial [3] was supported by a research grant from cord blood registry $(\mathrm{CBR} \circledast)$.

\section{Conflict of interest}

The authors have no relevant conflicts of interest to report related to this chapter. 


\section{Author details}

Linda Baumgartner ${ }^{1}$, Michael Seidman ${ }^{2}$, Deborah Lamontagne ${ }^{3}$, Ernest Moore ${ }^{4}$, David Shook ${ }^{5}$, Steven Messina ${ }^{6}$ and James Baumgartner ${ }^{7 *}$

1 Clarke School for Hearing and Speech, and Little HEARoes, Orlando, Florida, USA

2 Department of Otorhinolaryngology, College of Medicine, Advent Health Celebration and University of Central Florida, Orlando, Florida, USA

3 University of Tennessee, Memphis Tennessee, USA

4 University of North Texas, Denton, Texas, USA

5 Advent Health Orlando, Orlando, Florida, USA

6 Department of Radiology (Neuroradiology), Mayo Clinic College of Medicine, Rochester, MN, USA

7 Department of Neurological Surgery, College of Medicine, Advent Health Orlando and University of Central Florida, Orlando, Florida, USA

*Address all correspondence to: lbaumgartenr@clarkeschools.org

\section{IntechOpen}

(C) 2019 The Author(s). Licensee IntechOpen. This chapter is distributed under the terms of the Creative Commons Attribution License (http://creativecommons.org/licenses/ by/3.0), which permits unrestricted use, distribution, and reproduction in any medium, provided the original work is properly cited. (cc) BY 


\section{References}

[1] Lin FR, Niparko JK, Ferrucci L. Hearing loss prevalence in the United States. Archives of Internal Medicine. 2011;171:1851-1852

[2] Mehra S, Eavey RD, Keamy DG Jr. The epidemiology of hearing impairment in the United States: Newborns, infants and adolescents. Otolaryngology and Head and Neck Surgery. 2009;140:461-472

[3] Baumgartner LS, Moore E, Shook D, Messina S, Day MD, Green J, et al. Safety of autologous umbilical cord blood therapy for acquired sensorineural hearing loss in children. Journal of Audiology and Otology. 2018;22(4):209-222

[4] Shibata SB, Shearer AE, Smith RJH. Genetic sensorineural hearing loss. In: Flint PW, Haughey BH, Lund VJ, Niparko JK, Robbins DT, Thomas JR, editors. Cummings OtolaryngologyHead and Neck Surgery. 6th ed. Philadelphia, PA: An Imprint of Elsevier; 2014. pp. 2285-2300

[5] Canalis RF, Lambert PR. The Ear: Comprehensive Otology. 1st ed. Philadelphia, PA: Lippincott, Williams and Wilkins; 2000

[6] Smith RJ, Bale FJ Jr, White KR. Sensorineural hearing loss in children. Lancet. 2005;365:879-890

[7] Seidman MD, Bai U, Khan MJ, Murphy MP, Quirk WS, Castora FJ, et al. Association of mitochondrial DNA deletions and cochlear pathology: A molecular biologic tool. The Laryngoscope. 1996;106(6):777-783

[8] Bai U, Seidman MD. A specific mitochondrial DNA deletion $\left(\mathrm{mtDNA}^{4977}\right)$ is identified in a pedigree of a family with hearing loss. Hearing Research. 2001;154:73-80
[9] Seidman MD, Khan MJ, Tang WX, Quirk WS. Influence of lecithin on mitochondrial DNA and age-related hearing loss. OtolaryngologyHead and Neck Surgery Journal. 2002;127(3):138-144

[10] Prockop DJ. Marrow stromal cells as stem cells for nonhematopoietic tissues. Science. 1997;276:7-14

[11] Cox CS Jr, Baumgartner JE, Harting MT, Worth LL, Walker PA, Shah SK, et al. Autologous bone marrow mononuclear cell therapy for severe traumatic brain injury in children. Neurosurgery. 2011;65:588-600

[12] Vahidy FS, Rahbar MH, Shu H, Rowan PJ, Bambhroliya AB, Savitz SI. Systematic review and metanalysis of bone marrow-derived mononuclear cells in animal models of ischemic stroke. Stroke. 2016;47:1632-1639

[13] Savitz SI, Mistra V, Kasam M, Junija $\mathrm{H}$, Cox CS Jr, Alderman S. Intravenous autologous bone marrow mononuclear cells for ischemic stroke. Annals of Neurology. 2011;70:59-69

[14] White SV, Czisch CE, Han MH, Plant CE, Harvey AT, Plant GW. Intravenous transplantation of mesenchymal progenitors distributes solely to the lungs and improve outcomes in cervical spinal cord injury. Stem Cells. 2016;34:1812-1825

[15] van Velthoven CT, Dzietko M, Wenddland MR, Derugin N, Faustino J, Heijnen CG, et al. Mesenchymal stem cells attenuate MRI-identified injury, protect white matter and long-term functional outcome after neonatal focal stroke in rats. Journal of Neuroscience Research. 2017;95:1225-1236

[16] van Velthoven CT, Sheldon RA, Kavelaars A, Derugin N, Vexler ZS, Willement HO, et al. Mesenchymal stem 
cell transplantation attenuates brain injury after neonatal stroke. Stroke. 2018;44:1426-1432

[17] Revoltella RP, Papini S, Rosellini A, Michleini M, Franscechini V, Ciroba A, et al. Cochlear repair by transplantation of human cord blood CD-133+ to nod-scid mice deafened with kanamycin and noise. Cell Transplantation. 2008;17:665-678

[18] Bettini S, Franceschini V, Astolfi L, Simoni E, Massanti B, Martini A, et al. Human mesenchymal stromal cell therapy for damaged cochlea repair in nod-scid mice deafened with kanamycin. Cytotherapy. 2018;20:189-203

[19] Choi MY, Yeo SW, Park KH. Hearing restoration in a deaf animal model with intravenous transplantation of mesenchymal stem cells derived from human umbilical cord blood. Biochemical and Biophysical Research Communications. 2014;427:629-636

[20] DaCosta V, O'Grady G, Jackson L, Kaylie D, Raynor E. Improvement in sensorineural hearing loss after cord blood transplant in patients with mucopolysaccharidosis. Archives of Otolaryngology - Head \& Neck Surgery. 2012;138:1071-1076

[21] Sharma A, Nash AA, Dorman M. Cortical development plasticity and reorganization in children with cochlear implants. Journal of Communication Disorders. 2009;42(4):272-279

[22] Sharma A, Glick H. Cortical neuroplasticity in hearing loss: Why it matters in clinical decision-making for children and adults. Hearing Review. 2018;25(7):20-24

[23] Lieu JEC, Tye-Murray N, Karzon RK, Piccirillo JF. Unilateral hearing loss is associated with worse speechlanguage scores in children. Pediatrics. 2010;125(6):1346-1355
[24] Holtmaat A, Wilbrecht L, Knott GW, Welker E, Svoboda K. Experiencedependent and cell-type-specific spine growth in the neocortex. Nature. 2006;441(7096):979-983

[25] Huttenlocher PR, de Courten C. The development of synapses in the striate cortex of man. Human Neurobiology. 1987;6(1):1-9

[26] Huttenlocher PR, Dabholkar AS. Regional differences in synaptogenesis in the human cortex. The Journal of Comparative Neurology. 1997;387(2):167-178

[27] Ponton CW, Eggermont JJ. Of kittens and kids: Altered cortical maturation following profound deafness and cochlear implant use. Audiology \& Neuro-Otology. 2001;6(6):363-380

[28] Sharma A, Dorman MF. Central auditory development in children with cochlear implants: Clinical implications. Advances in Oto-Rhino-Laryngology. 2006;64:66-88

[29] Oh SH, Kim CS, Kang EJ, Lee DS, Lee HJ, Chang SO, et al. Speech perception after cochlear implantation over a 4-year time period. Acta OtoLaryngologica. 2003;123(2):148-153

[30] Geers AE. Factors influencing spoken language outcomes in children following early cochlear implantation. Advances in Oto-Rhino-Laryngology. 2006;64:50-65

[31] Geers AE, Moog JS, Biedenstein J, Brenner C, Hayes H. Spoken language scores of children using cochlear implants compared to hearing agemates at school entry. Journal of Deaf Studies and Deaf Education. 2009;14(3):371-385

[32] Holt RF, Svirsky MA. An exploratory look at pediatric cochlear implantation: Is earliest always best? Ear and Hearing. 2008;29(4):492-511 
[33] Gilley PM, Sharma A, Mitchell TV, Dorman MF. The influence of a sensitive period for auditory-visual integration in children with cochlear implants. Restorative Neurology and Neuroscience. 2010;28(2):201-218

[34] Meredith MA, Krylywy J, McMillan AH, Malhotra S, Lum-Tai R, Lomber SG. Crossmodal reorganization in the early deaf switches sensory, but not behavioral roles of auditory cortex. Proceedings of the National Academy of Sciences of the United States of America. 2011;108(21):8856-8861

[35] Bola L, Zimmerman M, Mostkowski P, Jednorog K, Marchewka A, Rutkowski $\mathrm{P}$, et al. Task-specific reorganization of the auditory cortex in deaf humans. Proceedings of the National Academy of Sciences of the United States of America. 2017;114(4):E600-E609

[36] Zhang JS, Guan AL, Zhang XG, Beydoun H, Seidman M, Elisevich K, et al. Cortical electrical suppression of tinnitus and modulation of its related neural activity. The New Zealand Medical Journal. 2010;123(1311):77-167

[37] Zhang JS, Guan ZL, Ramachandran V, Dunford J, Seidman M, Bowyer SM, et al. Electrical modulation of tinnitus related activity. Seminars in Hearing. 2008;29(4):313-326

[38] Meredith MA, Lomber SG. Somatosensory and visual crossmodal plasticity in the anterior auditory fields of early deaf cats. Hearing Research. 2011;280(1-2):38-47

[39] Seidman MD, Van de Water T. Pharmacologic manipulation of the labyrinth with novel and traditional agents delivered to the inner ear. Ear, Nose, and Throat Journal. 2003;82(4):276-304

[40] Zanetti D. Characterization of hearing loss in children with mucopolysaccharidosis. In: Hatzopuolos
S, editor. An Excursus into Hearing

Loss. Rijeka, Croatia: Intech Open; 2018.

DOI: 10.5772/intechopen.74196

[41] Simmons MA, Bruce IA, Penney S, Wraith E, Rothera MP. Otorhinolaryngological manifestations of the mucopolysaccharidoses. International Journal of Pediatric Otorhinolaryngology. 2005;69(5):589-595

[42] Taylor M, Khan S, Stapleton M, Wang J, Chen J, Wynn R, et al. Hematopoietic stem cell transplantation for mucopolysaccharidoses: Past, present and future. Biology of Blood and Marrow Transplantation. 2019. DOI: 10.1016/j.bbmt2019.02.012

[43] Mukherjee P, Miller JH, Shimony JS, Philip JV, Nehra D, Snyder AZ, et al. Diffusion-tensor MR imaging of gray and white matter development during normal human brain maturation.

AJNR - American Journal of Neuroradiology. 2002;23:1445-1456

[44] Devine SM, Cobbs C, Jennings M, et al. Mesenchymal stem cells distribute to a wide range of tissues following systemic infusion into nonhuman primates. Blood. 2003;101:2999-3001

[45] Gholamrezanezhad A, Mirpour $\mathrm{S}$, Bagheri $\mathrm{M}$, et al. In vivo tracking of 11in-oxine labeled mesenchymal stem cells following infusion in patients with advanced cirrhosis. Nuclear Medicine and Biology. 2011;38:961-967

[46] Krueger TEG, THorek DLJ, Denmeade SR, Isaacs JT, Brenned WN. Concise review: Mesenchymal stem cell-based drug delivery: The good, the bad, the ugly and the promise. Stem Cells Translational Medicine. 2018;7:651-663

[47] Leyendecker A Jr, Gomez Pinheiro CC, Amano MT, Bueno DF. The use of human mesenchymal stem 
cells as therapeutic agents for the in vivo treatment of immune-related diseases: A systemic review. Frontiers in Immunology. 2018;9:2056. DOI: 10.3389/fimmu.2018.02056

[48] Fierabracci A, Del Fattore A, Luciano R, Muraca M, Teti A, Muraca $M$. Recent advances in mesenchymal stem cell immunomodulation: The role of microvesicles. Cell Transplantation. 2015;24:133-149

[49] Gebler A, Zabel O, Seliger B. The immunomodulator capacity of mesenchymal stem cells. Trends in Molecular Medicine. 2012;18:128-134

[50] Uccelli A, Moretta L, Pistoia V. Mesenchymal stem cells in health and disease. Nature Reviews. Immunology. 2008;8:726-736

[51] Zhang QZ, Su WR, Shi SH, WilderSmith P, Xiang AP, Wong A, et al. Human gingiva derived mesenchymal stem cells elicit polarization of $\mathrm{m} 2$ macrophages and enhance wound healing. Stem Cells. 2010;28:1856-1868

[52] Le Blanc K, Tammik L, Sundberg B, Haynesworth SE, Ringden O.

Mesenchymal stem cells inhibit and stimulate mixed lymphocyte cultures and mitogenic responses independently of the major histocompatibility complex. Scandinavian Journal of Immunology. 2003;57:11-20

[53] Singer NG, Caplan AI. Mesenchymal stem cells: Mechanisms of inflammation. Annual Review of Pathology. 2011;6:457-478

[54] VandenBosch LS, Reh TA.

Epigenetics in neuronal regeneration. Seminars in Cell \& Developmental Biology. 2019. DOI: 10.1016/j. semcdb.2019.04.001

[55] Liang X, Ding Y, Zhang Y, Tse HF, Lian Q. Paracrine mechanisms of mesenchymal stem cell-based therapy:
Current status and perspectives. Cell

Transplantation. 2014;23(9):1045-1059 
Section 2

\section{Implantable Hearing Aids}





\title{
Congenital Aural Atresia: Hearing Rehabilitation by Bone-Anchored Hearing Implant (BAHI)
}

\author{
Giampietro Ricci, Arianna Di Stadio, Valeria Gambacorta \\ and Antonio Della Volpe
}

\begin{abstract}
Auris atresia (AA) is a congenital pathology characterized by aplasia or hypoplasia of the external ear with associated middle ear malformation. The AA has a different degree of severity, and the severe form of the disorder presents no identifiable ear canal (complete atresia) and absence or significative underdevelopment of the middle ear structures. Sometimes AA is associated with a malformation of the ear called "microtia." The alterations of the external auditory canal and of the middle ear structures are responsible for the conductive hearing loss which affects the patients. The hearing restoration procedures may recreate a normal external and middle ear anatomy to favor the recovery of the hearing function, or the surgeon may simply restore the hearing capacity through bypassing the malformed structures by boneanchored hearing implants (BAHIs). The restoring of normal anatomy is generally associated with episode of restenosis of the external ear canal due to bony regrowth. The formulation of a therapeutic strategy may be supported by using Jahrsdoerfer classification to identify the severity of malformation. In the chapter we discuss various bone anchoring prostheses currently used (Baha, Ponto, Alpha2 by Sophono, Bonebridge) and the results that can be obtained by the use of these implants.
\end{abstract}

Keywords: congenital aural atresia, hearing loss, hearing restoration, bone-anchored hearing implant, memory function

\section{Introduction}

Congenital aural atresia (CAA) is a congenital malformation of the ear that causes both esthetic and functional impairments. The malformation presents different severities of impairment; CAA may be the only malformation in the body or be associated with other malformation as observed in syndromic patients.

CAA is a failure in the ear development that happens in the first gestation' weeks; the failure may be complete by affecting the external and the middle ear or partial, as, for example, a stenotic external canal with normal middle ear structures.

In all cases, the hearing function is impaired, despite having different hearing threshold.

Our group showed that independently from the severity of the hearing impairment, children with hearing loss present a reduction of the memory function and scholar abilities [1], so the restoration of hearing function should be considered 
the first goal in CAA followed, eventually, before the adolescent age by an esthetic reconstruction of the external ear [2]. In 1992 Jahrsdoerfer et al. [3] proposed a CT grading system for CAA that was shown to correlate with postoperative hearing outcomes, as a supporting method during the decisional process of treatment of CCA malformation; the suggestion of the author was to treat the high score of malformation with external canal bone reconstruction and eventually ossiculoplasty, while in the case of lower scores, the bone-anchored hearing implants (BAHIs) were the most appropriate treatment.

Other more recent scoring systems as the Lübeck score have been proposed; this system is based on strong analyses conducted on high-resolution CT scan. The proposed method used a 16-score grading for addressing to the best ear implant [4] that has the same efficacy as the Jahrsdoerfer et al. classification [3].

In this chapter we discuss the CCA management with BAHIs by illustrating the different implants available on the market and the implantation method and finally reporting the results that we obtained in more than 10 years of experience with these systems.

\section{Congenital auris atresia (CCA)}

Congenital aural atresia is an ear malformation that may have different severity and may affect the external ear only or the middle ear too. The ear malformation may be a single problem or be part of a syndromic picture. An altered development of the first and second branchial arches and the first branchial cleft may be responsible for the CCA [5].

Schuknecht [6] classified four degree of severity based on the combination of high-resolution computer tomography (CT) scan and surgical findings: (1) Type
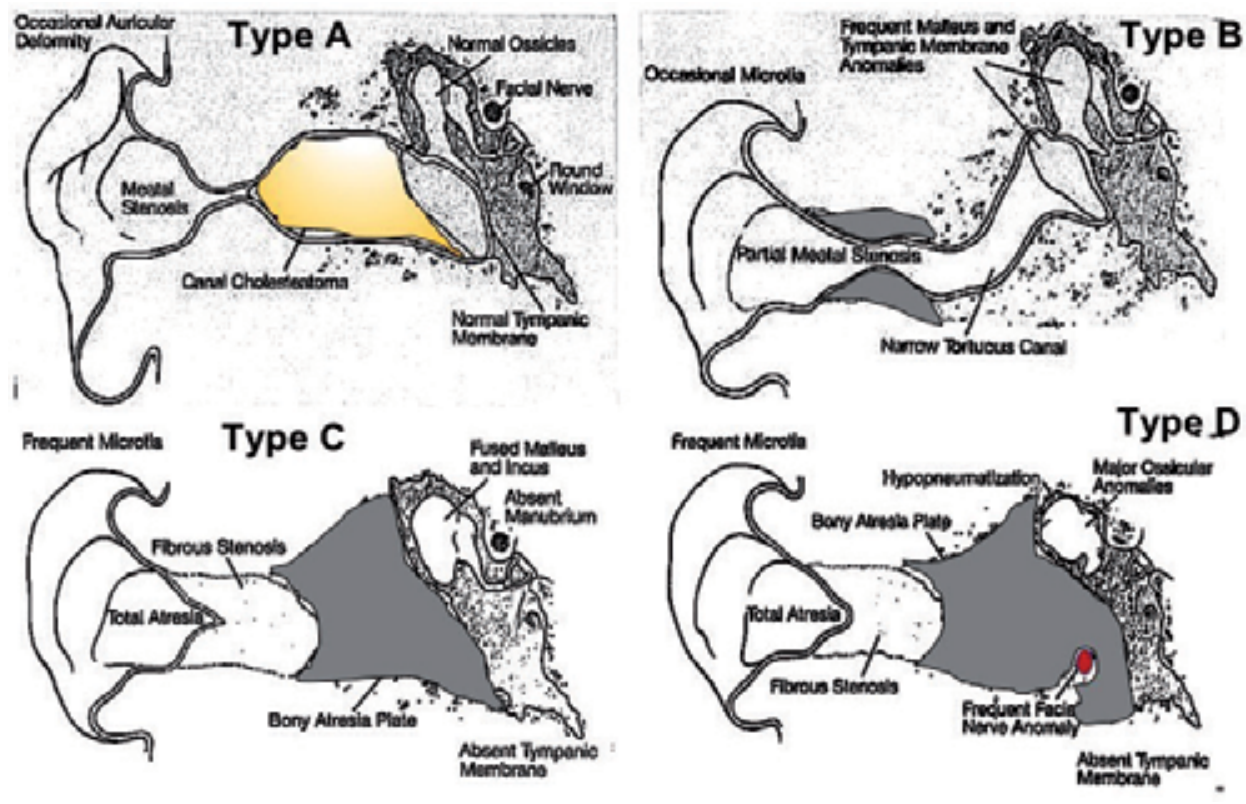

Figure 1.

The image shows the four types of CAA as described by Schuknecht. In Type A, the yellow indicates the presence of the cholesteatoma behind the meatal stenosis. The gray areas in Types $B, C$, and D represent the portion with bone atresia. The red dot in type D shows the aberrant exit of facial nerve. 
A: narrowing of the fibrocartilaginous canal and presence of cholesteatoma distal to the stenotic area. (2) Type B: narrowing and tortuosity of fibrocartilaginous and bony part of the canal, commonly associated with abnormal tympanic membrane and malleus. (3) Type C: complete atresia with different combination of fibrous tissue and bone. Typically, malleus and incus are fused together, the manubrium and the tympanic membrane are missing, and the stapes is mobile. (4) Type D: totally atresia with decreased pneumatization of mastoid. More severe ossicular anomalies than type $\mathrm{C}$ and frequently the facial nerve being aberrant (Figure 1).

Other authors classified the CAA; one of the most famous is Weerda [7] that classified these malformations in three types (A, B, and C), while Altmann described a histopathological classification correlating the severity of CAA [8] by identifying three categories: mildly, moderately, and severely malformed types. Many authors have since modified this classification system, further subclassifying type II based on the surgical findings and functional outcome [9].

\section{Embryology and etiopathogenesis of CAA}

The mandibular (I) and the hyoid (II) branchial arches contribute to the auricular development, and both may be involved in the etiopathogenesis of CAA. Auricular pinna starts to develop between the third to sixth weeks of embryonic life, when hillocks appear on the arches, and its formation is complete at the fourth month of gestation. The basis of tragus, the helical root, and the superior part of the helix comes from the anterior three hillocks, derived from the first arch. The posterior hillock that derives from second arch is responsible for the formation of the antihelix, antitragus, and lobule. The middle ear cavity derives from the first pharyngeal arch starting from 4 weeks of gestation. The pinna develops around the external meatus which becomes canalized at week 28 of embryologic life. At 8 weeks the middle ear cleft is formed, and the cavity is complete developed at 30 weeks. The first arch cartilage generates malleus and incus by 8 weeks of gestation that start to ossify at the 4 months of pregnancy. From the second arch, cartilage comes out the stapes except the medial lamina of the footplate which derives from the otic capsule [10]. At week 9, ectodermal cells proliferate, fill the meatus lumen, and form the "meatal plug" (MP); then on week 10, the MP extends in a disclike fashion by following a horizontal plane, and the internal part of MP starts to thin for generating the future tympanic membrane. At the same time, the plug in the proximal portion of the neck starts to be resorbed. At week 13 the MP is in contact with the primordial malleus, and this contact will contribute to the thinness of internal part of MP that will create the tympanic membrane at week 15 . At week 16 the external ear canal is fully patent but still narrow and curved. At week 18 the meatus is fully extended and starts its opening that will be completed at 28 weeks [11].

Any type of adverse event that occurs during the 4 and 25 weeks of gestation and interrupts one of more of these developments may be responsible for one of the different types of CAA. The adverse event may be related to genetic aberrations, vascular accident (fetal hypoxia), teratogenic substances (aminoglycoside antibiotics, hydantoin, alcohol, nicotine, herbicides), maternal infection (rubella, Cytomegalovirus, measles, hepatitis, toxoplasmosis, lues), and maternal metabolic disease (deficiency of thyroid hormone or diabetes) [12].

CAA may be a single malformation or be associated with other malformations as in the case of oto-facial dysostosis (Treacher-Collins syndrome, Goldenhar syndrome), craniofacial dysostosis (Crouzon syndrome, Apert syndrome), otocervical dysostosis (Klippel-Feil syndrome, Wildervanck syndrome), oto-skeletal 
dysostosis (Van der Hoeve-De Klein syndrome, Albers-Schonberg disease), and chromosomal syndromes (trisomy 13, 18, 21, and 18q syndrome) [2].

\section{Indications to use BAHI in patients with CAA}

CAA is predominantly unilateral (ca. 70-90\%) and the malformation mostly affects the right ear, perhaps because this side can suffer more frequently of hypoperfusion even rather that the left side in which the heart is located and that generally has a pressure $10 \mathrm{mmHg}$ higher to the right side. The incidence of ear malformations is approximately 1 in 3800 newborns. Some children may present a bilateral CAA, when the malformation is not an isolated disease, but it is contextualized in a syndrome as, for example, CHARGE syndrome, in which children are affected by bilateral atresia up to $60 \%$ of cases [13].

Patients may be affected from different severity of external and middle ear malformation (Figure 1): due to the severity we can identify different forms of hearing loss. CAA typically results in conductive hearing loss (CHL) in 80-90\% of the cases with the remaining patients demonstrating a sensorineural hearing loss (SNHL) component [10]. The CHL is typically in the moderate hearing loss range of 40-60 dB; this is the range in which BAHIs work better (Figure 2).

In the case of children suffering from unilateral CAA [14] and sensorineural hearing loss (10-20\% of children with CAA), BAHI may be used for restoring the hearing function if the contralateral normal hearing function is preserved [15-17].

We use to utilize the Jahrsdoerfer et al. method during our decisional process for identifying the most appropriate surgery technique that has to be used. The authors proposed a CT grading system for CAA that was shown to correlate with postoperative hearing outcomes; based on the scores reached in the preliminary

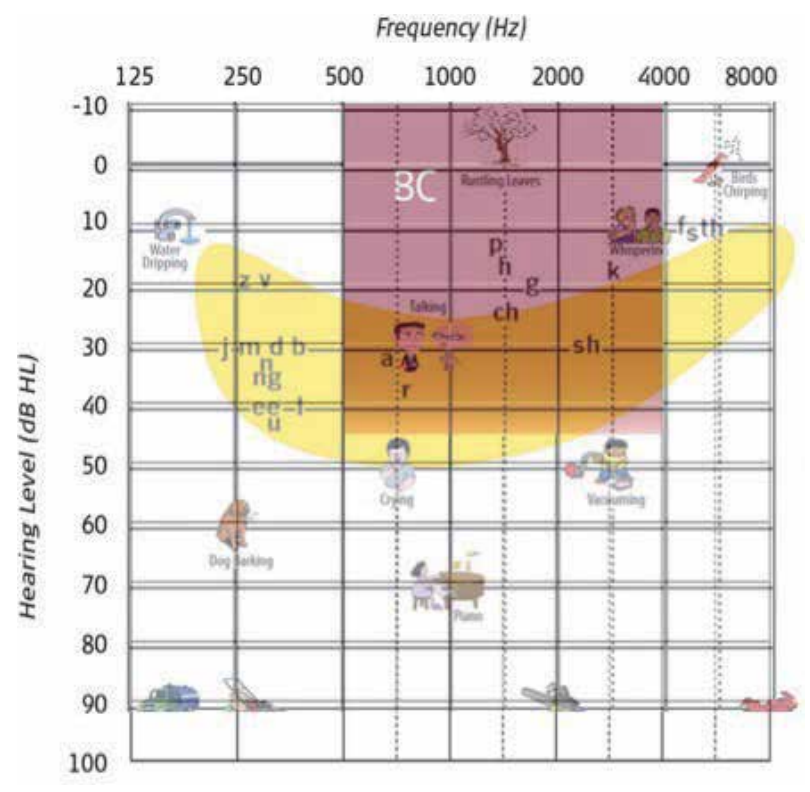

Figure 2.

The back image shows the ideal condition to use BAHI, while the supra-impressed yellow banana illustrates the distribution of vocal frequency. CHL that presents an auditory threshold within $45 \mathrm{~dB}$ may benefit from a BAHI because the implant guarantees a good recover of auditory functions in the range between 500 and $4000 \mathrm{~Hz}$. 
patient evaluation, the authors proposed different surgical options. In the case of high scores (better option), a canaloplasty with eventual ossiculoplasty may be a good option for the treatment of CAA, while, in the case of low scores, the authors' suggestion is to use a BAHI [3]. Specifically, the authors concluded that the patient with a score of $8-9$ is a very good candidate ( $80 \%$ chance to reach postoperative PTA threshold of $30 \mathrm{~dB}$ or lower) for surgical reconstruction of the auditory canal and the middle ear, while a score of $5 / 6$ or less disqualifies patients for surgery.

Furthermore, they identified that syndromic patients rarely present a grade higher than 6/7 and, in general, are poor surgical candidates (Table 1 ).

Anyway even in the case of success (Table 2), a surgical reconstruction presents a several postsurgical complication as stenosis of the new external auditory canal (15-20\%), recurrent otitis externa (10\%), sensorineural hearing loss $(5 \%)$ cholesteatoma $(2-4 \%)$, and facial nerve injury $(0.1 \%)$, and often a hearing aid is necessary to allow a good hearing function $[15,18-21]$.

\begin{tabular}{lc}
\hline Parameter & Points \\
\hline Stapes present & 2 \\
\hline Oval window open & 1 \\
\hline Middle ear space & 1 \\
\hline Facial nerve normal & 1 \\
\hline Malleus/incus complex presence & 1 \\
\hline Mastoid well pneumatized & 1 \\
\hline Incus-stapes connection & 1 \\
\hline Round window normal & 1 \\
\hline Appearance external ear & 1 \\
\hline Total available points & 10 \\
\hline
\end{tabular}

Table 1.

The Jahrsdoerfer grading system of candidacy for CAA repair.

\begin{tabular}{lcc}
\hline Authors and year & Number of patients & Mean PTA $\leq$ 30 dB HL \\
\hline Lambert, 1988 & 16 & $12(67 \%)$ \\
\hline Bellucci, 1981 & 71 & $39(55 \%)$ \\
\hline Mattox and Fisch, 1986 & 11 & $5(45 \%)$ \\
\hline De la Cruz et al., 1985 & 56 & $41(73 \%)$ \\
\hline Schuknecht, 1989 & 50 & $15(50 \%)$ \\
\hline Jahrsdoerfer, 1992 & 126 & $61(48 \%)$ \\
\hline Murphy et al., 1997 & 19 & $4(21 \%)(20 \mathrm{~dB})$ \\
\hline Teufert and De la Cruz, 2004 & 115 & $55(48 \%)$ \\
\hline Digoy and Cueva, 2007 & 54 & $27(50 \%)$ \\
\hline El-Hoshy, 2008 & 40 & $26(65 \%)$ \\
\hline Yellon et al., 2011 & 19 & $8(45 \%)$ \\
\hline Nadaraja et al., 2013 & 390 & $235(60.3 \%)$ \\
\hline
\end{tabular}

Table 2.

The experiences of different authors on the surgical reconstruction of external ear canal are reported. 
Based on our experience and according to the Jahrsdoerfer study, we can affirm that patients with CAA and a score of $<7$ rarely benefit from an external and middle ear reconstruction with good functional results. In such cases, the implantation of a BAHI is a viable option for obtaining a stable, satisfactory, and long-term result in terms of hearing function recovery [18]. In conclusion we think that BAHI could be quite always the best method for restoring hearing function in patients with CAA, even in the case of high score in the Jahrsdoerfer grading system.

\section{Available product}

The hearing restoration by bone stimulation did not obtain an immediate success; in fact in 1920 the first electronic implantable device was the preferred method, and bone stimulation prostheses were considered as the last alternative. Furthermore, after the Second World War, with the advent of transistor, the electronic prosthesis became smaller and more manageable, similar to the actual hearing aids. Anyway, in the recent decades, thanks to the new discoveries which better explained the physiologic stimulation of the inner by bone stimulation and to the improved technologies that miniaturize the systems and make the system implantable (BAHI Figure 3), the system is widely used for the treatment of hearing impairments $[21,22]$. BAHI solved the old problems related to the external bone stimulation as the difficulties in maintaining constant position and correct pressure on the mastoid and, in addition, the reduced bone stimulation due to the skin impedance [23, 24].

In the light of multiple observations and experiments, it was concluded that the bone pathway is a type of natural sound conduction and that the sound characteristics are normal and quite similar to that conducted by air.

The BAHI solves the problems of the traditional retro-auricular bone stimulation as poor performance due to inadequate contact between the vibrator and the skin, pain or decubitus at the site of contact, and poor esthetic acceptance of arch prostheses by combining a pin implanted in the temporal bone with an external transducer; the vibration of the transducer on the bone induces a stimulation of the inner ear cells with consequently sound perception.

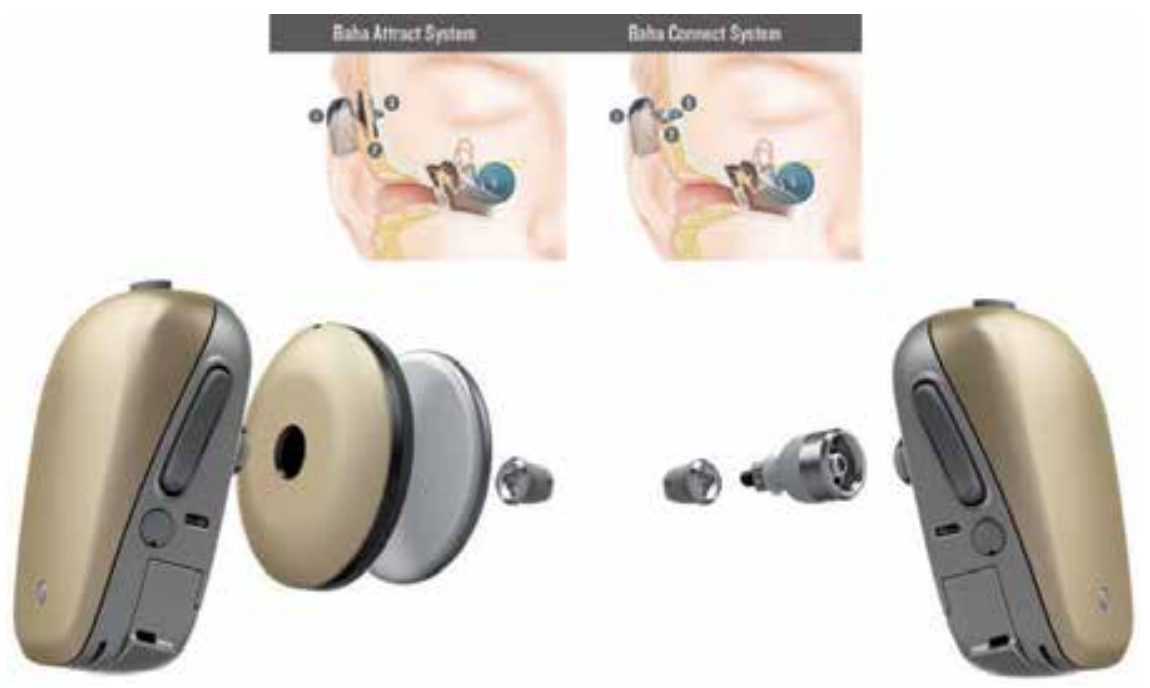

Figure 3 .

On the left side, the transcutaneous BAHI, and on the right side, the percutaneous. 
Congenital Aural Atresia: Hearing Rehabilitation by Bone-Anchored Hearing Implant (BAHI) DOI: http://dx.doi.org/10.5772/intechopen.88201

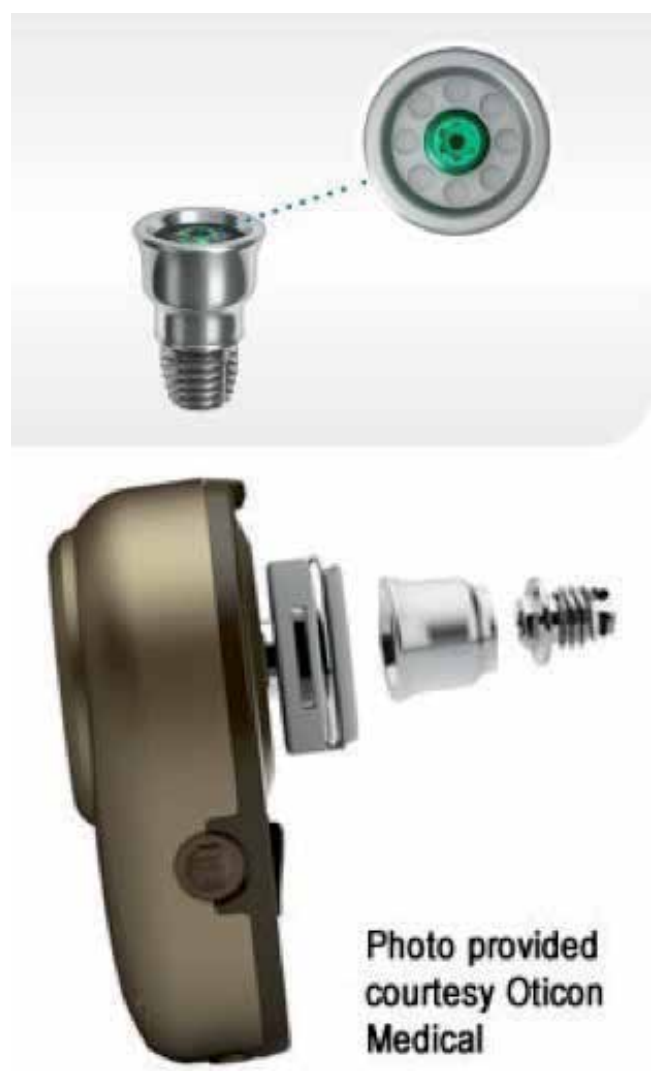

Figure 4.

Percutaneous BAHI (PONTO) produced by Oticon Medical.
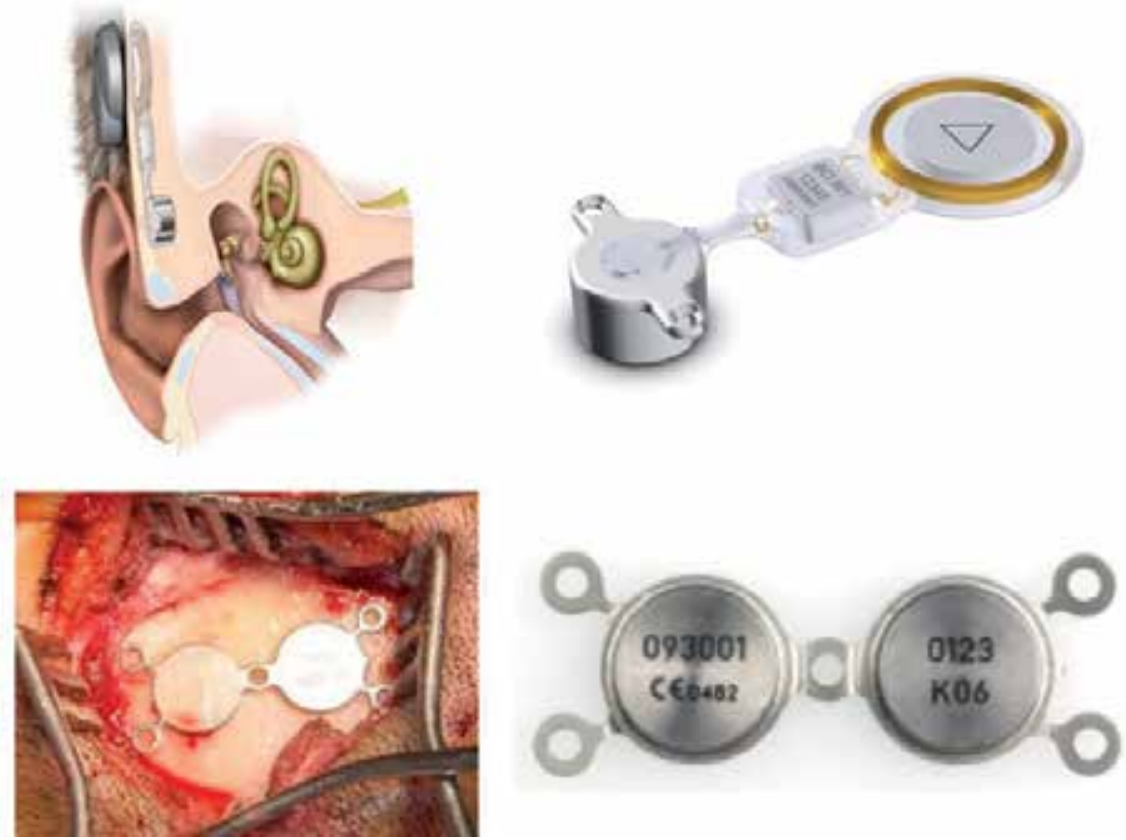

Figure 5

On the top of the image, a semi-implantable transcutaneous device BONEBRIDGE (Medel) and on the bottom another model of transcutaneous BAHI ALPHA 2 by SOPHONO (Medtronic). 
The coupling between the pin and the transducer determines the type of BAHI: percutaneous or transcutaneous. Both methods of implant need a surgery.

The difference between the transcutaneous and the percutaneous system is the way in which the titanium screw is placed related to the skin plan; in the first one, the skin is surgically open, and the abutment is placed directly on the mastoid; then the surgical opening is closed by suturing the skin [25]. The percutaneous system instead consists of a titanium implant placed "through" the skin by perforating it [26].

All BAHI systems are composed of three main components:

1. An internal titanium fixture that is surgically anchored to the temporal bone behind the ear

2. An external abutment that is connected to the implant at the time of surgery

\section{An external sound processor that is snapped on to the abutment}

All systems currently available on the market present these characteristics: high amplification power, working independently in the presence/absence of the ear canal and middle ear, a direct bone transmission giving a clear sound, may be tested preoperatively, and all systems being quite similar in terms of comfort [27-29] (Figures 3-5).

\section{Temporal bone anatomy and BAHI}

Surgeons have to keep in mind the normal anatomy of temporal bone because it is helpful for remembering the anatomic landmarks when severe malformations occurred in the patient that should be implanted.

When CAA is associated with microtia, the main anatomic landmarks are the zygomatic process (image 7 sagittal plane view); in fact this structure is generally quite preserved also in the case of craniofacial malformation.

In the case of the absence of zygomatic process, the squamosal suture should be identified as alternative landmarks (Figure 6).

\section{SAGITTAL PLANE VIEW}

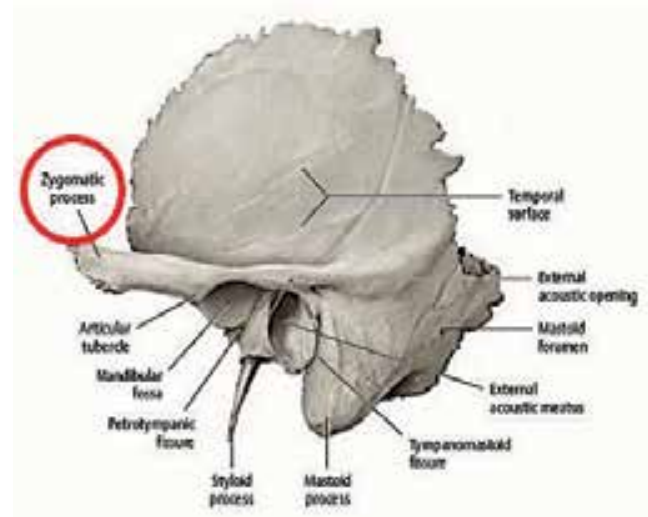

CORONAL PLANE VIEW

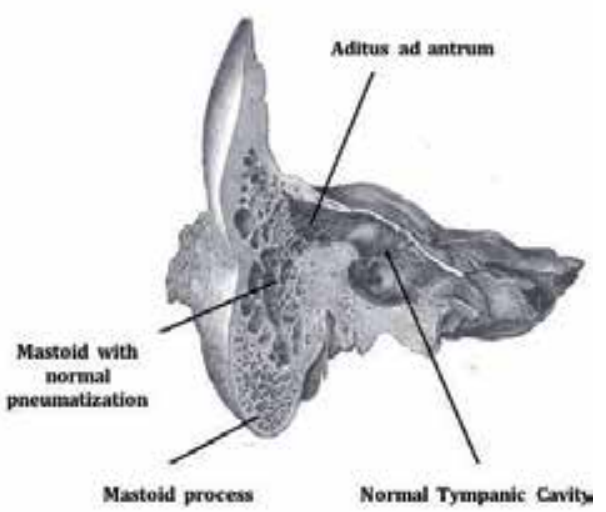

Figure 6.

The normal anatomy of human temporal bone in sagittal and coronal views. The red circle indicates the zygomatic process, the only landmark that may be present in the case of CAA associated with craniofacial malformation (Figures 7-9). 

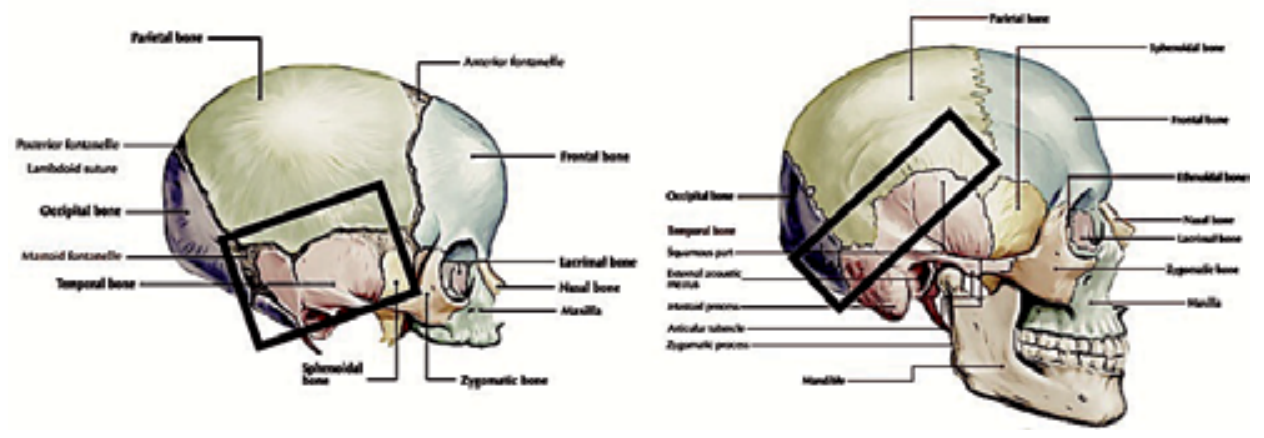

Figure 7.

The image shows the squamosal suture in a normal head. In the left side the cranium of a child before the closure of the suture; in the right the squamosal suture as appears in the adult head.
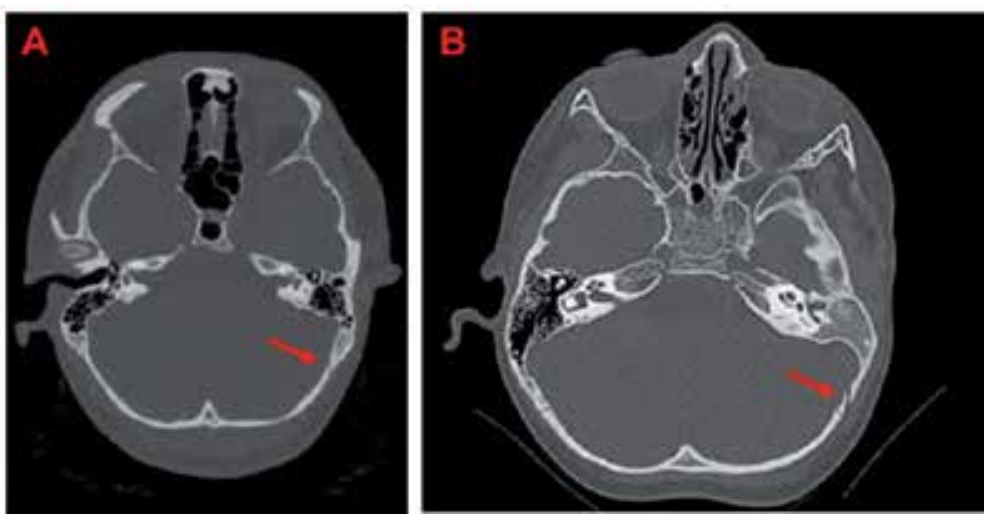

Figure 8.

CT scan high-resolution images. (A) Type D CAA with preservation of the normal mastoid pneumatization. The red arrow indicates the squamosal suture. (B) Type D CAA with absence of mastoid pneumatization; the red arrow shows the clear presence of the squamosal suture that is still not closed.
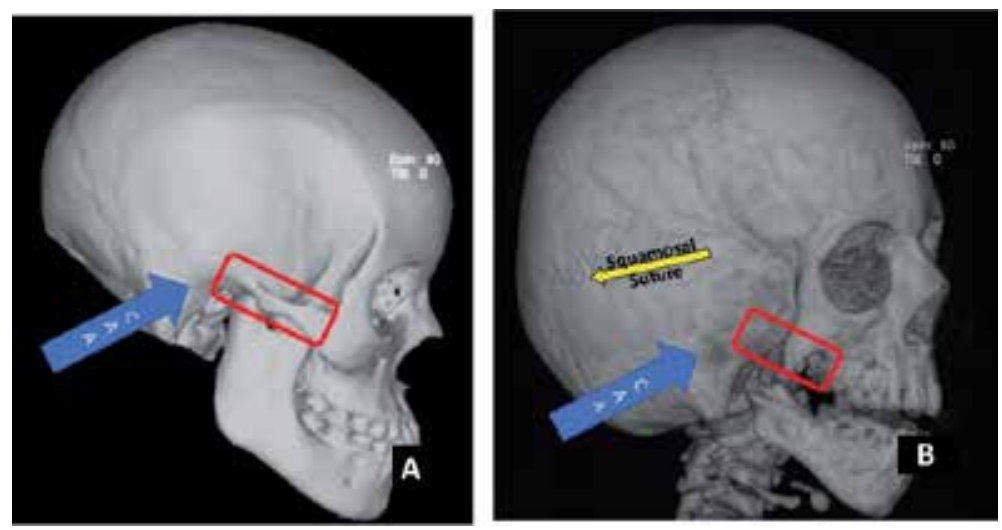

Figure 9.

(A) CAA in a non-syndromic patient with complete preservation of zygomatic arch anatomy (red rectangle). (B) CAA in a syndromic patient; the zygomatic arch is extremely malformed (red rectangle) and cannot be used as a surgical anatomic landmark. The squamosal suture is well identifiable (yellow arrow) and can be used as an alternative to zygomatic arch. 


\section{Surgical method of implant and timing}

The FDA recommends to apply the device in children not younger than 5 years. Authors say that the ideal age for implanting a BAHI is between 2 and 4 years, because at this age the thickness of retromastoid bone $(2.5-3 \mathrm{~mm})$ allows to perform a totally safe surgery, without risk of damaging the dura. Additionally, regarding the age of implant, we have to consider that children need to possess a sufficient manual dexterity to maintain the device and a good psychological tendency to follow the suggestions of parents and caregivers. We usually correct hearing aids before 5 years by using an elastic band (Softband).

The Tjellström et al. technique, proposed in 1977, suggest the creation of a thin muscle-cutaneous flap. The main disadvantage is an altered vascularization that may lead to infection and wound healing problems, dysesthesia in the retroauricular area and alopecia. For solving these problems, a less invasive technique by using a linear retro-auricular incision has been proposed and currently is the mostly used. The main benefit of this new technique is the good preservation of the vascularization in the surgical area that allows to avoid all the problems of muscle-cutaneous flap (infection, healing problems, dysesthesia, etc.). Another suggested low invasive technique is based on the removal of a very small piece of skin in the area in which the titanium implant should be placed. The skin removed has the same dimension of a biopsy. This technique also presents the advantages of the liner incision, due to the reduced trauma on tissue and vascular structures [30].

\subsection{BAHA ${ }^{\circledR}$ (https://www.cochlear.com/it/home) and PONTO ${ }^{\circledR}$ (https://www. oticonmedical.com/it)}

See Figures 10 and 11.

\subsection{Alpha 2 (www.sophono.com)}

The Alpha 2 by Sophono is another bone-anchored prosthesis without percutaneous screw and consists of a processor that is coupled to the skin transcutaneously with a titanium component implanted subcutaneously, containing two magnets.
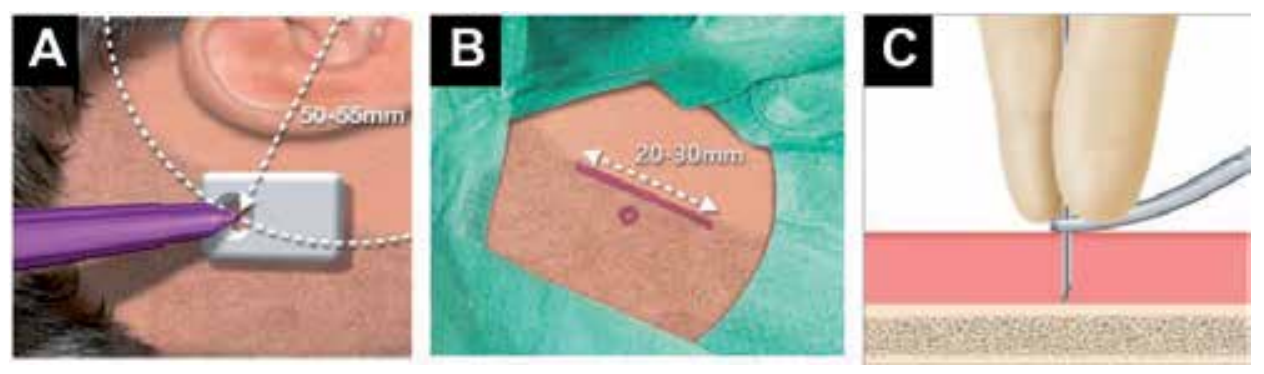

Figure 10.

(A) BAHI model is placed on the skin exactly in the area where it will be placed. After considering the distance from the superior margin of the external auditory canal, a point is deigned on the skin as landmark. A meter is used to measure the distance from the external auditory canal where the BAHI that is approximately $50-55 \mathrm{~mm}$ is placed. This distance is necessary to avoid the prosthesis from touching the pinna. (B) A line parallel to the point previously identified is then designed in the skin of the retro-auricular area. (C) After using the BAHI model for designing the location of the implant, a blue mytilene solution is injected for delineating on the mastoid bone the lodgment of the implant. 

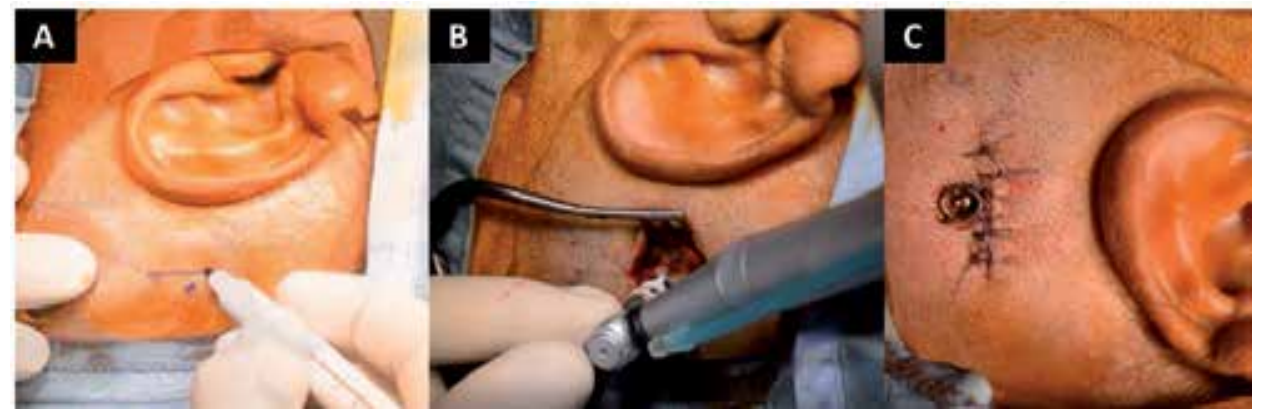

Figure 11.

(A) By using a dermographic pen, the incision line is designed on the skin. (B) After surgical incision the lodgment for the bone anchorage is performed by using a drill. (C) The anchorage after the skin closure with head in vertical position. Final result. Live surgery: https://wwwyoutube.com/watch?v=Pz2qZxzkV1I.
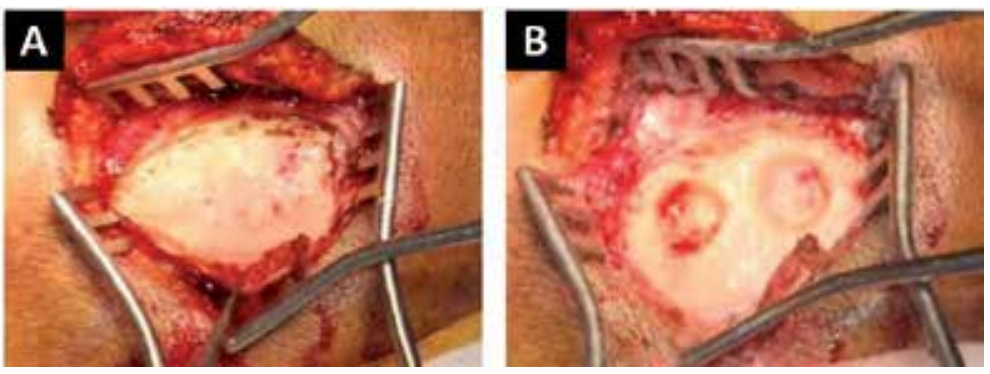

Figure 12.

(A) Musculocutaneous flap with exposure of mastoid (B) using a cutting burr creation of lodgment area.
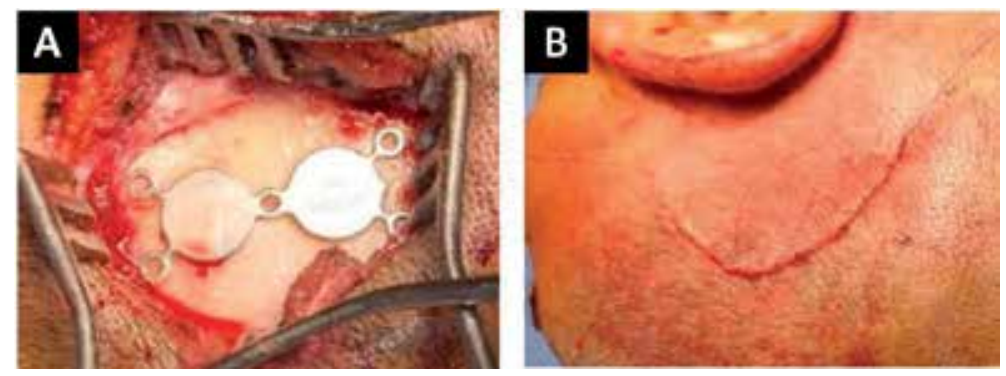

Figure 13.

(A) BAHI is placed and fixed with titanium screw. (B) Continuous cutaneous suture to guarantee sterility. Live surgery traditional method: https://wwwyoutube.com/watch?v=AagsHvgsmDs. Live surgery simplified method: https://www.youtube.com/watch?v=gJM1jt8W_rI.

The magnetic attraction allows to hold the processor in place and to transmit acoustic energy [31]. The limits of this kind of device are represented by energy loss through the skin layer and possible discomfort and complications due to soft tissue pressure [21] (Figures 12 and 13).

\subsection{Bonebridge (https://www.medel.com/hearing-solutions/bonebridge)}

The Bonebridge by MED-EL is a semi-implantable bone conduction auditory system comprising a processor that is coupled transcutaneously to a titanium component and silicone implanted subcutaneously. The transducer, lodged in the 
mastoid bone, transmits mechanical vibrations directly to the inner ear jumping middle and outer ear. The conduction system is light $(\sim 10 \mathrm{~g})$, and it has an area of $\sim 8.7 \mathrm{~mm}$ (height) $\times 15.8 \mathrm{~mm}$ (diameter). Two titanium screws-responsible for the vibrations transduced to the system-are located to a distance of around $24 \mathrm{~mm}$ between them.

Bonebridge is the unique BAHI with active mechanism; the information analyzed by the audio-processor are sent to the antenna and transmitted through the skin to the system. It converts the received signals into mechanical vibrations, which are transmitted to the inner ear by bone conduction. The transductor is surgically positioned at the seno-dural angle. However, due to its slightly bulky internal implant, the most optimal location for placing BB implant should be carefully selected preoperatively using 3D reconstruction software [32].

As compared to percutaneous BCI, Bonebridge's transcutaneous technology enables the avoidance of several complications including skin reaction, growth of skin over the abutment, implant extrusion, and wound infection.

$\mathrm{BB}$ presents the same risks of the other BAHIs (skin infection and skin necrosis), and currently nobody described severe complications by using this implant. We suggest to use a double flap for minimizing the skin trauma and improving the outcomes (Figures 14 and 15).
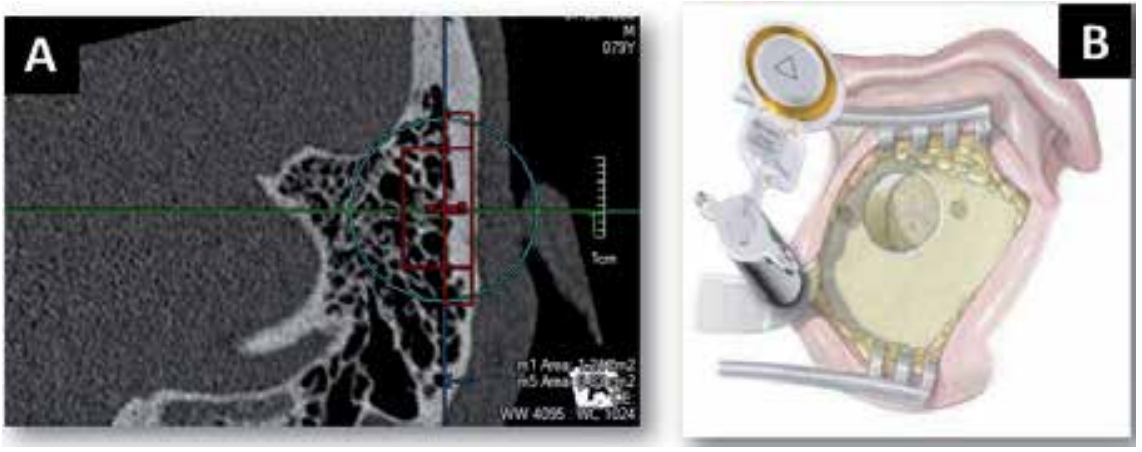

Figure 14.

(A) Measurement of temporal bone thickness before surgery and (B) lodgment for BAHI created posteriorly to the pinna.

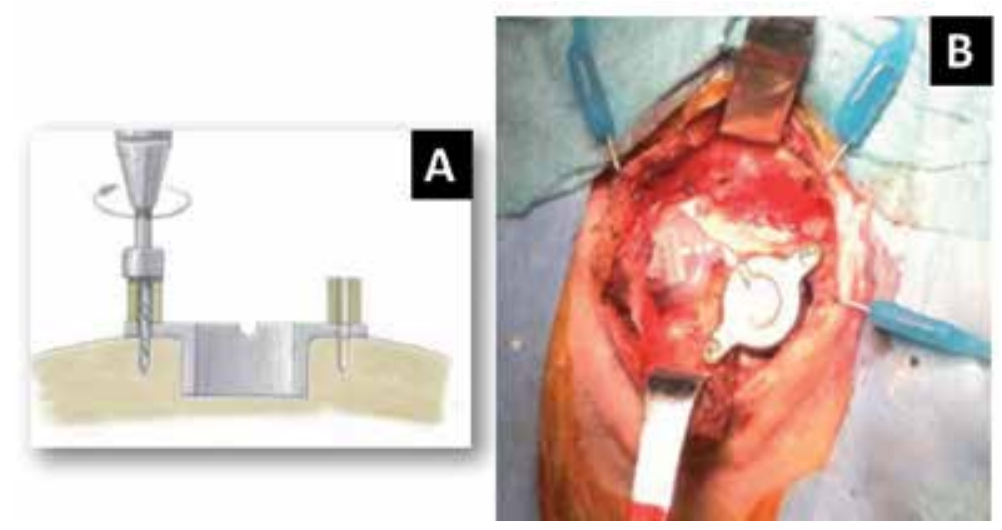

Figure 15.

(A) After lodged BAHI is fixed to the temporal bone with a screw and (B) way in which the implant appears before the closure of the skin. Live surgery: https://wwwyoutube.com/watch?v=Wlh6YxEnJl8. 


\section{Our results}

We widely use BAHIs both in the case of aural atresia (singular or bilateral form) $[1,15,21]$ and as treatment of single side deafness [1], and our results are supported by other authors' observations [16-19, 29, 30].

We observed that BAHI not only improved the hearing abilities of children in noise condition but also their dictation capacity during the school activity $[1,15,21]$.

We compared children wearing BAHI immediately after the implant and then 3 months later they were implanted, and we noticed that their abilities in speech perception increased time by time by reaching the same scores observed in healthy children after 3 months [1]. In addition, we analyzed the short and working memory abilities in children with BAHI, before and after implantation, and we identified that both these memory functions improved by restoring the hearing with BAHIs. The memory abilities, as the speech perception done, increased time by time by overlapping the score obtained by healthy children in the same age range [1]. Finally, we observed that children after being implanted with BAHI improved their school scores and increased their relationship with friend and environment [21].

Reported complications are slight skin problems as localized irritation and hypertropic scare around the titanium implant. The skin inflammation may be explained as an immune answer to an external body (the screw). In the literature the rates go from 5 to $7.5 \%$ for skin regrowth and from $1.3-10 \%$ for extrusion of the abutment [15].

\section{Conclusions}

We think that BAHIs are a very good solution for restoring hearing abilities. Although we have a wide experience on children, due to the excellent results obtained on these patients, in terms of speech discrimination but much more for the impressive benefit obtained in memory function, we suggest BAHIs as treatment of hearing loss in adults' population too.

As we have shown in our chapter, BAHIs are simple to implant, with very low side effects, and their surgery is poor and time-consuming. We speculate that due to the new theories on BAHIs' stimulation of the inner ear [33-37], these implants could be a valid alternative to the traditional hearing aids in slight-mild form of asymmetric hearing loss; we suggest BAHIs not only in the case of conductive hearing loss (CHL) but also in the sensorineural form (SNHL).

\section{Conflict of interest}

The authors declare no conflict of interest. 


\section{Author details}

Giampietro Ricci ${ }^{1}$, Arianna Di Stadio ${ }^{2 *}$, Valeria Gambacorta ${ }^{1}$ and Antonio Della Volpe ${ }^{2}$

1 Otolaryngology Department, University of Perugia, Perugia, Italy

2 Otolaryngology Department, Santobono-Posilipon Children Hospital, Cochlear Implant Unit, Naples, Italy

*Address all correspondence to: arianna.distadio@unipg.it

\section{IntechOpen}

(C) 2019 The Author(s). Licensee IntechOpen. This chapter is distributed under the terms of the Creative Commons Attribution License (http://creativecommons.org/licenses/ by/3.0), which permits unrestricted use, distribution, and reproduction in any medium, provided the original work is properly cited. (cc) BY 


\section{References}

[1] Di Stadio A, Dipietro L, Toffano R, Burgio F, De Lucia A, Ippolito V, et al. Working memory function in children with single side deafness using a bone-anchored hearing implant: A case-control study. Audiology \& NeuroOtology. 2018;23(4):238-244. DOI: $10.1159 / 000493722$

[2] Elder LM, Zur KB, editors. Congenital Malformation of Head and Neck. New-York: Springer; 2014. pp. 23-66

[3] Jahrsdoerfer RA, Yeakley JW, Aguilar EA, et al. Grading system for the selection of patients with congenital aural atresia. The American Journal of Otology. 1992;13(1):6-12

[4] Frenzel H, Sprinzl G, Widmann G, Petersen D, Wollenberg B, Mohr C. Grading system for the selection of patients with congenital aural atresia for active middle ear implants. Neuroradiology. 2013;55:895-911

[5] Abdel-Aziz M. Congenital aural atresia. The Journal of Craniofacial Surgery. 2013;24(4):e418-e422. DOI: 10.1097/SCS.0b013e3182942d11

[6] Schuknecht HF. Congenital aural atresia. The Laryngoscope. 1989;99(9):908-917

[7] Weerda H. Chirurgie der ohrmuschel: Verletzungen, defekte und anomalien. Stuttgart: Thieme; 2004. pp. 105-226

[8] Altmann F. Congenital atresia of the ear in man and animals. The Annals of Otology, Rhinology, and Laryngology. 1955;64(3):824-858

[9] Declau F, Offeciers F, Van de Heyning P. Classification of the nonsyndromal type of meatalatresia. In: Devranoglu I, editor. Proceedings of the XVth World Congress of
Otorhinolaryngology Head and Neck Surgery: Panel Discussions. Istanbul, Turkey. 1999. pp. 135-137

[10] Beahm EK, Walton RL. Auricular reconstruction for microtia:

Part I. anatomy, embryology, and clinical evaluation. Plastic and Reconstructive Surgery. 2002;109(7):2473-2482

[11] Stilianos E. Kountakis Encyclopedia of Otolaryngolog, Head and Neck Surgery y. Switzerland: Edition Springer Link; 2013

[12] Kelley PE, Scholes MA. Microtia and congenital aural atresia. Otolaryngologic Clinics of North America. 2007;40(1):61-80

[13] Bartel-Friedrich S, Wulke C. Classification and diagnosis of ear malformations. GMS Current Topics in Otorhinolaryngology, Head and Neck Surgery. 2007;6:Doc05

[14] Zhang L, Gao N, Yin Y, et al. Bone conduction hearing in congenital aural atresia. European Archives of OtoRhino-Laryngology. 2016;273:1697-1703

[15] Ricci G, Della Volpe A, Faralli M, Longari F, Gullà M, Mansi N, et al. Results and complications of the Baha system (bone-anchored hearing aid). European Archives of Oto-RhinoLaryngology. 2010;267(10):1539-1545. DOI: $10.1007 / \mathrm{s} 00405-010-1293-0$

[16] Sprinzl G, Lenarz T, Ernst A, et al. First European multicenter results with a new transcutaneous bone conduction hearing implant system: Short-term safety and efficacy. Otology \& Neurotology. 2013;34:1076-1083

[17] Bianchin G, Bonali M, Russo M, Tribi L. Active bone conduction system: Outcomes with the Bonebridge 
transcutaneous device. ORL: Journal for Otorhinolaryngology and Its Related Specialties. 2015;77:17-26

[18] Bouhabel S, Arcand P, Saliba I. Congenital aural atresia: Boneanchored hearing aid vs. external auditory canal reconstruction. International Journal of Pediatric Otorhinolaryngology. 2012;76(2):272277. DOI: 10.1016/j.ijporl.2011.11.020

[19] Lo JF, Tsang WS, Yu JY, Ho OY, $\mathrm{Ku}$ PK, Tong MC. Contemporary hearing rehabilitation options in patients with aural atresia. BioMed Research International. 2014;2014:761579

[20] Yu JK, Wong LL, Tsang WS, Tong MC. A tutorial on implantable hearing amplification options for adults with unilateral microtia and atresia. BioMed Research International. 2014;2014:703256

[21] Ricci G, Della Volpe A, Faralli M, Longari F, Lancione C, Varricchio AM, et al. Bone-anchored hearing aids (Baha) in congenital aural atresia: Personal experience. International Journal of Pediatric

Otorhinolaryngology. 2011;75(3):342346. DOI: 10.1016/j.ijporl.2010.11.027

[22] Reinfeldt S, Hakansson B, Taghavi H, Eeg-Olofsson M. Bone conduction hearing sensitivity in normal-hearing subjects: Transcutaneous stimulation at BAHA vs BCI position. International Journal of Audiology. 2014;53:360-369

[23] Gavilan J, Adunka O, Agrawal S, et al. Quality standards for bone conduction implants. Acta OtoLaryngologica. 2015;135(12):1277-1285

[24] Bento RF, Kiesewetter A, Ikari LS, Brito R. Bone-anchored hearing aid (BAHA): Indications, functional results, and comparison with reconstructive surgery of the ear. International
Archives of Otorhinolaryngology. 2012;16(3):400-405. DOI: 10.7162/ S1809-97772012000300017

[25] Håkansson B, Eeg-Olofsson M, Reinfeldt S, Stenfelt S, Granström G. Percutaneous versus transcutaneous bone conduction implant system: A feasibility study on a cadaver head. Otology \& Neurotology. 2008;29(8):1132-1139. DOI: $10.1097 /$ MAO.0b013e31816fdc90

[26] Fuchsmann C, Tringali S, Disant $F$, et al. Hearing rehabilitation in congenital aural atresia using the bone-anchored hearing aid: Audiological and satisfaction results. Acta Oto-Laryngologica. 2010;130(12):1343-1351

[27] Pross S, Layton A, Tong K, Lustig L. Cost of placement and complications associated with osseointegrated bone conduction hearing prostheses: A retrospective analysis of Medicare billing data. Otology \& Neurotology. 2014;35:476-481

[28] Monini S, Bianchi A, Talamonti R, Atturo F, Filippi C, Barbara M. Patient satisfaction after auditory implant surgery: Ten-year experience from a single implanting unit center. Acta Oto-Laryngologica. 2016;5:1-9

[29] DI Giustino F, Vannucchi P, Pecci R, Mengucci A, Santimone R, Giannoni B. Bone-anchored hearing implant surgery: Our experience with linear incision and punch techniques. Acta Otorhinolaryngologica Italica. 2018;38(3):257-263. DOI: 10.14639/0392-100X-1694.

[30] Siegert R, Kanderske J. A new semi-implantable transcutaneous bone conduction device: Clinical, surgical, and audiologic outcomes in patients with congenital ear canal atresia. Otology \& Neurotology. 2013;34:927-934 
[31] Zanetti D, Di Berardino D. A bone conduction implantable device as a functional treatment option in unilateral microtia with bilateral stapes ankylosis: A report of two cases. American Journal of Case Reports. 2018;19:82-89. DOI: 10.12659/ AJCR.904907

[32] Stenfelt S. Inner ear contribution to bone conduction hearing in the human. Hearing Research. 2015;329:41-51. DOI: 10.1016/j.heares.2014.12.003

[33] Sohmer H, Freeman S, Geal-Dor M, Adelman C, Savion I. Bone conduction experiments in humans-A fluid pathway from bone to ear. Hearing Research. 2000;146(1-2):81-88

[34] Brinker T, Stopa E, Morrison J, Klinge P. A new look at cerebrospinal fluid circulation. Fluids Barriers CNS. 2014;11:10. DOI: 10.1186/2045-8118-1110. eCollection 2014

[35] Eeg-Olofsson M, Stenfelt S, Granström G. Implications for contralateral bone conducted transmission as measured by cochlear vibrations. Otology \& Neurotology. 2011;32(2):192-198. DOI: 10.1097/ MAO.0b013e3182009f16

[36] Di Stadio A. Which factors to induce hearing loss in professional musicians? Extensive literature review and histopathology findings can answer it. Hearing, Balance and Communication (IHBC). 2017;15(2):63-71

[37] Nadaraja GS, Gurgel RK, Chang KW. Hearing outcomes of atresia surgery versus osseointegrated bone conduction device in patients with congenital aural atresia: A systematic review. Otology \& Neurotology.

2013;34(8):1394-1399 



\title{
Chapter 6
}

\section{The Esteem ${ }^{\circledR}$, Fully Implantable Middle Ear Device}

\author{
Maurizio Barbara and Simonetta Monini
}

\begin{abstract}
The active middle ear implant (AMEI) may be considered, in selected cases, a valid alternative to conventional hearing aids (cHA) for rehabilitation of bilateral sensorineural hearing loss (SNHL). Since 2007, at the Implanting Centre of the University Hospital Sant'Andrea in Rome, 43 subjects underwent surgery for application of the Esteem ${ }^{\circledR}$, after ascertaining by CT scan its feasibility for allocating its transducers within the mastoid space. The surgical procedure is longer than for the other AMEI, and the switch on of the device is usually performed 4-6 weeks after surgery. All the Esteem ${ }^{\circledR}$ implantees underwent a pre- and postoperative assessment via pure tone and speech audiometry with headset in a soundproof booth. Along with the recommended population with moderate-to-severe hearing loss, subjects also with a worse hearing loss (severe or severe-to-profound) were selected for this implant for different reasons. The auditory outcome in label and off-label implantees was analyzed. Complications included the need for a minor revision, due to middle ear fibrosis, or for an explant that was followed by ossicular reconstruction with return to a cHA (two subjects), cochlear implantation (five subjects) or no alternative solution (one case).
\end{abstract}

Keywords: Esteem, active middle ear implant, fully-implantable device, sensorineural hearing loss, hearing rehabilitation

\section{Introduction}

Active middle ear implants (AMEI) represents today one of the possible rehabilitative solutions for hearing-impaired individuals. These devices utilize, for their function, a vibratory effect instead of the classical, physiological air-conducted sound stimulation. This natural sound transmission way, if warranting the perfect solution under normal hearing conditions, usually generates several negative issues on a hearing-impaired ear, going from an insufficient stimulation to the presence of distortions when the conventional hearing amplifiers, i.e. hearing aids (HA), are in use. In fact, despite their striking technological improvement over the last decades (advanced miniaturization, open-fitting systems, sophisticated digital signal processing software, and wide connectivity), the latter are rarely showing to be optimal for the patients' auditory needs while generating distorted amplification issues (feedback, occlusion effect, and insufficient high-frequency gain). As a consequence, the number of HA users is far from equaling the purchased devices worldwide. Another playing factor is due to the scarce impact on some quality of life issues (manual difficulty, stigma, pathology, or reduced caliber of the external 
auditory canal, earmold allergies, and impossibility to use them during water contact, physical activities, and overnight, while sleeping).

The advent of AMEI with the stimulation pathway different from the normal (or amplified) sound, i.e., the electromechanical one, has paved the way for the possible theoretical resolution of the above-mentioned annoying cHA-related issues. In fact, these devices are directly coupled to middle or inner ear elements (ossicular chain or part of it, round window membrane) for achieving close-field cochlear stimulation, and should theoretically overcome most of the issues deriving from the obligatory acoustic over-stimulation that needs to be delivered by an external amplifier, such as a cHA. Moreover, all of them leave the external ear canal free, so as to improve the auditory and physical occlusion issues, as well as the compatibility with external ear canal or middle ear pathologies.

In relation to the presence of an external component, the AMEI can be distinguished in semi- or fully implantable devices, this latter term inherently meaning that all their components remain completely invisible under the skin. At the present time, only two fully implantable devices are available for the rehabilitation of sensorineural hearing loss (SNHL): the Carina ${ }^{\circledR}$ (Cochlear, Lane Cove, Australia) and the Esteem ${ }^{\circledR}$ (Envoy Medical Corporation, St. Paul, MN, USA). Both devices have in common the complete allocation inside the skull, while carrying at the same time some major differences, as listed in Table 1.

This last appealing factor is surely playing an important role for the decisionmaking process while counseling with a possible candidate, but it would not be wise to consider it as primary factor for its choice, and anyhow never replacing a preoperative use or trial with a best-fitted last-generation digital $\mathrm{cHA}$.

At Sant'Andrea University Hospital, Rome, Italy, an extensive rehabilitative program for hearing impairment has been applied since nearly 15 years, mostly aiming at the resolution of different types and severity of conductive, mixed and SNHL. In particular, from 2007, a series of subjects affected by bilateral SNHL have undergone Esteem ${ }^{\circledR}$ surgical application. The Esteem ${ }^{\circledR}$ (Envoy Medical, St Paul, MN, USA) middle ear implant has recently been reported to be beneficial for individuals affected by moderate-to-severe bilateral SNHL, with low morbidity and complication rate $[1,2]$. This device works via a piezoelectric vibratory stimulus on the stapes and thereafter to the inner ear fluids, also vibrating the intact eardrum that plays as natural microphone. Following this physiological route, a natural sound quality is likely to be perceived by preserving auricular filtering (at high frequencies) and leaving the ear canal open, thus eliminating the occlusion effect.

The aim of the present report is to highlight specific clinical issues including those that have emerged while following up the Esteem ${ }^{\circledR}$-implanted patients over several years. In particular, special attention is devoted to the surgical procedure; to the functional outcome, also in comparison with the performance with cHA, and its impact on the quality of life; to the battery, in terms of its variable duration and surgical replacement; and, finally, to the complication occurrence and rate.

\begin{tabular}{|c|c|c|c|c|}
\hline \multicolumn{5}{|c|}{ Essential features of fully implantable AMEI } \\
\hline & Microphone & Battery & Stimulation & $\begin{array}{l}\text { FDA approval (by } \\
\text { June 2019) }\end{array}$ \\
\hline Esteem & No & $\begin{array}{l}\text { Periodic surgical } \\
\text { change }\end{array}$ & Piezoelectric & Yes \\
\hline Carina & Yes & Daily recharge & Electromagnetic & No \\
\hline
\end{tabular}

Table 1.

Features of the two fully implantable hearing systems available today; FDA: Food and Drug Administration. 
The implantable components of the Esteem ${ }^{\circledR}$ are represented by a sound processor (SP), powered by a nonrechargeable battery, and two piezoelectric transducers, i.e., the sensor and the actuator (driver) (Figure 1).

The SP titanium case is housed in a temporal bone niche and receives the electrical signals from the sensor cemented to the incus body. After being processed, the signals are sent to the driver cemented to the stapes head, with a vibrational movement that generates the perilymphatic wave for cochlear stimulation

(Figure 2).

The Envoy Medical Company recommendations for candidacy include moderate to severe types of SNHL whilst its application is discouraged in patients with word recognition score (WRS) in quiet inferior to $40 \%$. Nevertheless, recent reports have shown that Esteem ${ }^{\circledR}$ may provide large amplification ranges up to $2-4 \mathrm{kHz}$, regardless of degree of hearing loss [3].

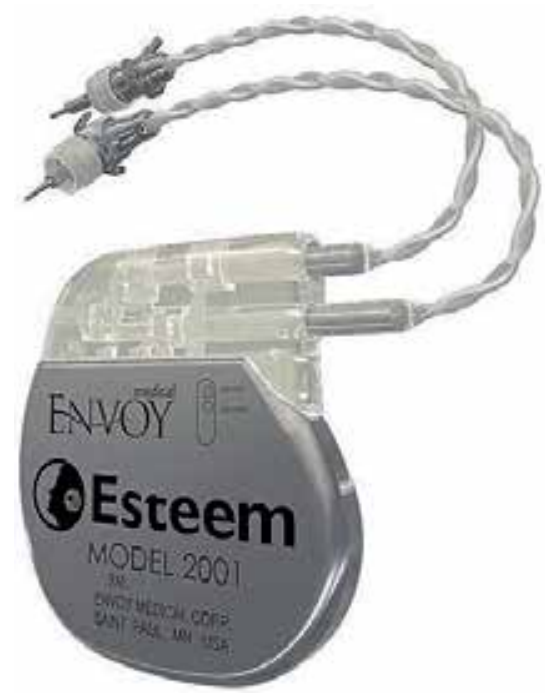

Figure 1.

The Esteem ${ }^{\circledR}$ middle ear implant. The sound processor is connected to the two transducers, namely the sensor and the driver.

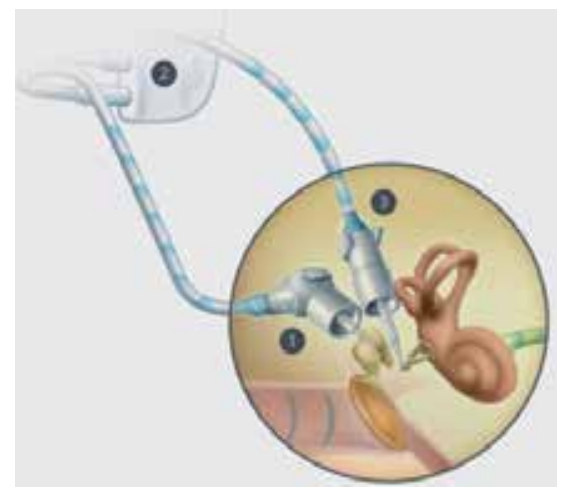

Figure 2.

The two transducers connected to the sound processor (2) are then placed in contact with the ossicular chain: The sensor (1) to the incus body and the driver (3) to the stapes head. 


\section{Material and methods}

All selected candidates were implanted with the Esteem ${ }^{\circledR}$ AMEI. Potential candidates were selected after a thorough audiometric assessment, which included pure tone and speech audiometry. In accordance with the Envoy Medical data, the advised threshold range is that reported in Figure 3, with a speech discrimination score better than $40 \%$.

Once the audiological clearance is given, a preliminary CT scan of the petrous bone is carried out to assess the feasibility of the procedure, in particular, if the space inside the mastoid cavity would be sufficient for housing the two transducers: the sensor on the incus body, by measuring the distance between the incus body and the sino-dural angle, and the driver on the stapes head, through a wide posterior tympanotomy, going posteriorly toward the sigmoid sinus region and lateral to the mastoid facial nerve course. At this point, all the information regarding use, time of use, and performances with a cHA is taken and thoroughly evaluated by speech audiometry in quiet and noise as well as by specific questionnaires.

The surgical procedure is performed under general anesthesia, with facial nerve monitoring and under hypotensive control all along the surgical steps, especially when cementing is taking place. The main steps of the surgical procedure are summarized as follows:

1. Identification of a flat, retro-auricular area in which a bony niche for the sound processor (SP) can be drilled. Accordingly, the skin incision is outlined and injected with vasoconstrictor solution.

2. A lazy-C retro-auricular incision, including skin and subcutaneous tissue, is carried out. After placement of self-retaining retractors, a large Palva flap is created and elevated with an anteriorly based pedicle. The SP bone well is then drilled by using large (6-8 $\mathrm{mm}$ ) cutting and diamond burs, ending up by drilling two small holes on both sides for securing the SP to the skull at the end of surgery by nylon thread.

3. An enlarged mastoidectomy is then drilled, completely exposing the presigmoid area, the sino-dural angle, and the inferior mastoid cell tip area.

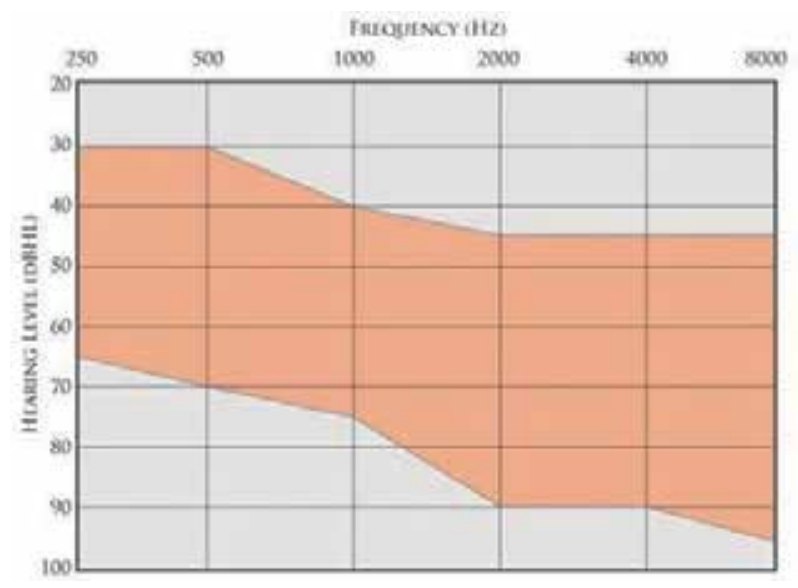

Figure 3.

The audiometric range of indication for the Esteem ${ }^{\circledR}$ fully implantable hearing device. 
4. Posterior epitympanectomy is then carried out until getting the complete exposure of the incus body and the malleus head, with the incudomalleolar joint.

5. Posterior tympanotomy is then drilled, thinning out—but keeping intact—the posterior buttress; drilling is continued until a gross trapezoidal-shaped opening is obtained at the expense of the chorda tympani nerve, which needs to be severed in all cases to avoid its eventual contact with the driver transducer. The lateral aspect of the posterior tympanotomy will correspond to the fibrous tympanic annulus. The superior corner of the posterior tympanotomy is drilled until obtaining an acute angle. The whole long process of the incus and the pyramidal eminence needs to be optimally visualized.

6. After placing a soft insert microphone-connected to a laser Doppler vibrometer (LDV) System - into the external auditory canal, two small reflectors are placed on the incus body and on the posterior crus of stapes for allowing the assessment of the normal mobility of the intact ossicular chain [4]. LDV, mounted on a second microscope, checks first the intact chain movement, which should mandatorily give normal values before continuing the procedure. LDV is a very accurate (G1 $10 \mathrm{j} 4 \mathrm{Km}$ ), noncontact instrument that works by comparing the frequency of the emitted laser diode light with the frequency of the reflected light of the moving object (Figure 4). Sound at 100-dB SPL and a sweep of 50 frequencies ranging from 125 to $8.000 \mathrm{~Hz}$ are used.

7. Separation of the incudostapedial joint is performed after gently removing the overlying mucosa.

8. By diode laser, then, part of the long incus process is sectioned.

9. At this point, removal by scraping of the mucosa over the stapes head is accomplished after dying the area with methylene blue, and finally drying it with low-watt laser beam.

10. A drop of EnvoyCem ${ }^{\circledR}$ (a bioglass type of biological cement) is then applied on the stapes head, so that a "precoat" is obtained.

11. Both sensor and drivers transducers are then attached to the Glasscock stabilizers screwed on the posterior edge of the mastoidectomy cavity and are then placed on the incus body and on the precoated stapes head, respectively.

12. MedCem ${ }^{\circledR}$ (an hydroxyapatite type of biological cement) is then composed and syringed into the mastoidectomy cavity for keeping and permanently fixing the transducers' bodies in place.

13. A small drop of EnvoyCem ${ }^{\circledR}$ is then placed to cement the tip of the driver on the precoated stapes capitulum, as well as between the sensor tip and the incus body, followed by creation of a new joint (detachment of the cemented sensor tip from the incus body).

14. Laser Doppler vibrometer (LDV) measurements are then performed, for testing both sensor and driver efficiency. 

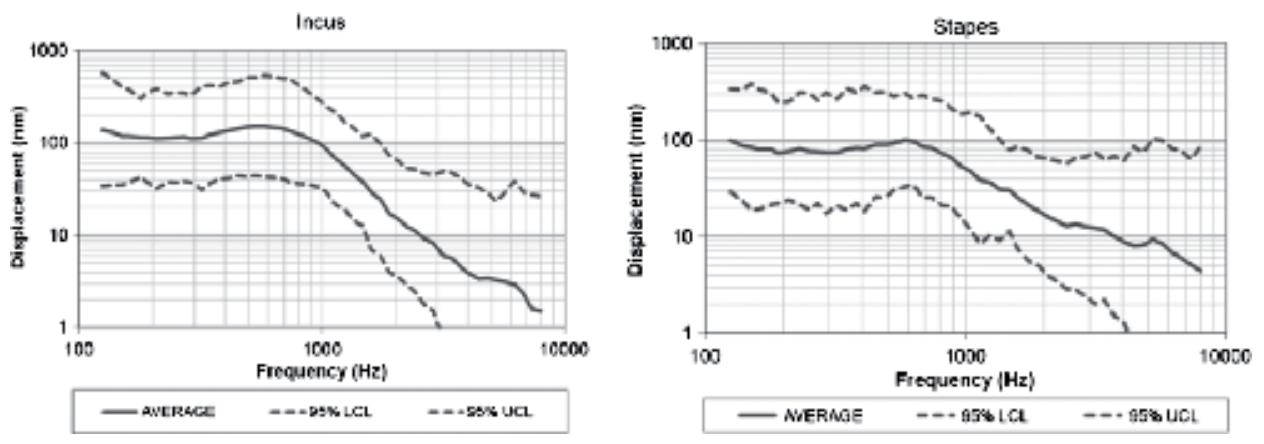

Figure 4.

Normative data regarding displacement of the incus (left) and stapes (right) when measured by laser Doppler vibrometry.

15. The SP is then put in place and attached to the sensor and driver cables. Final LDV measurements of the whole system are then carried out and precede the end of surgery with a three-layer closure.

A bandage is then wrapped around the head and kept for $24 \mathrm{~h}$. The implant is activated approximately 2 months after surgery, with the first fitting session, and later upgraded every 3 months during the first postoperative year.

Once required, the SP will be substituted via a minor surgical procedure, oftentimes performed under local anesthesia. The procedure entails a C-shaped, 5-cm long skin incision behind the SP that is subsequently exposed to allow first the disconnection from the cables of the two transducers, then their reconnection to the new SP.

\section{Results}

Eighty-two surgeries were performed in total, being 46 as primary intervention, 6 explantation, 4 revisions, and 27 battery changes. In three of the 46 primary surgeries, the procedure was interrupted due to low motility of the ossicular chain as shown by LDV in two cases and insufficient mastoid space in one case. So, the overall number of implants was 43. Implantation was always performed unilaterally, in the worse functioning ear of bilaterally hearing-impaired subjects; one subject was implanted while presenting with a unilateral severe SNHL. Bilateral application was completed in two subjects. Revision surgery was needed in three subjects for a developed fibrous tissue formation that impeded the normal movement of the ossicular chain and the transducers.

The outcome from Esteem ${ }^{\circledR}$ implantation has been analyzed taking into account several aspects, going from (a) the auditory improvement, (b) the comparison with the efficacy of a conventional hearing aid, (c) the patient's quality of life, and (d) the complication rate and the solutions for resolving these latter.

\section{Auditory improvement}

The mean postoperative pure tone threshold measured between 250 and $4000 \mathrm{~Hz}$ revealed an improvement of 5-10 dB in 9 subjects, 11-20 dB in 17 cases, $21-30 \mathrm{~dB}$ in 5 cases, and more than $30 \mathrm{~dB}$ in 3 cases. A -10/-20-dB deterioration was found in two subjects, and a $-5 /-10-\mathrm{dB}$ deterioration was found in three subjects. Overall, more than $80 \%$ of the implanted subjects showed an improvement in respect to the preoperative unaided bone conduction thresholds. More specifically, 
an improved threshold at $4 \mathrm{kHz}$ was measured in $62.5 \%$ of the implantees, while at $8 \mathrm{kHz}$, it was detected in $35 \%$ of them.

\subsection{Esteem vs. hearing aids}

The data relative to the efficacy of the Esteem ${ }^{\circledR}$ AMEI in comparison with the hearing aids has already been object of a previous publication [5]. In that report, two groups of subjects were identified: the first one with a moderate-to-severe SNHL and the other one with severe-to-profound SNHL, being theoretically beyond the indication range limit recommended by the manufacturer (off-label). In moderateto-severe cases, no statistical differences with the hearing aids were shown in the speech-in-noise tests, while subjectively, the implanted subjects reported a higher level of satisfaction in terms of a better quality of life. In the severe-to-profound subjects, similar results were obtained so as to also consider this range of hearing loss, a plausible threshold for implantation, as also outlined by other investigators [6]. The mean gain difference in favor of the Esteem ${ }^{\circledR}$ versus a cHA was equal to $13.1 \mathrm{~dB}$, thus very similar to what previously reported by Kraus et al. [6].

\subsection{Quality of life}

The data relative to the quality of life issue has already been reported [7]. This group of patients has been pooled together with other subjects receiving different types of auditory implant. In total, this survey has taken into consideration the 26 Esteem $^{\circledR}$ subjects who were all asked to fill different questionnaires including the General Glasgow Benefit Inventory, the visual analogue scale (VAS), and the abbreviated profile of hearing aid benefit (APHAB). The improvement of quality of sound was similar to the other implanted devices, whilst the score for quality of life was superior to both percutaneous bone conductive implants and to the Vibrant Soundbridge ${ }^{\circledR}$ (Medel, Innsbruck, Austria), equaling only that measured with the Bonebridge $^{\circledR}$ (Medel, Innsbruck, Austria).

\subsection{Complications}

It is important to distinguish two typologies of complications: one related to the surgical procedure, being inescapably part of it, and the other one as true complications. The surgical procedure implies the disconnection between incus and stapes so that an additional conductive component to the subject's hearing loss is always postoperatively evident. The other aspect regards the need for sacrificing the chorda tympani nerve so that a taste disturbance could be the consequence of it. In this regard, however, only a very limited number of subjects reported a permanent dysgeusia. Finally, it has also to be considered that the need to reconstruct the ossicular chain should an explantation be needed. Among the true complications, the need for explantation occurred in six subjects: for skin dehiscence (one subject), loud noise (one subject), tinnitus (one subject), and hearing threshold deterioration (three subjects). Four subjects required a surgical revision and in about $9 \%$ of the cases, a delayed transient facial palsy was observed, with remarks that were object of a previous publication [8].

\section{Discussion}

The Esteem ${ }^{\circledR}$ fully implantable AMEI represents a real alternative to conventional hearing aids when this latter one show to be inappropriate or unable 
to provide an efficient auditory amplification and in highly selected cases. More specifically, with a vibratory, direct stimulation of the anatomical structures located close to the cochlea, such as the stapes, footplate, or round window membrane, the delivered energy is much lower than that required for a cHA.

The activity of the Implanting Centre at Sapienza University has been displayed by the application of several types of bone conductive implants and AMEI, these latter as semi- and fully implantable devices. Among them, the Esteem ${ }^{\circledR}$ AMEI was specifically considered attractive for the invisibility as well as for the absence of an implanted microphone. This premise has allowed us to collect the largest European experience since 2007, with several subjects that have reached today a long-term use of the device so as to allow us to draw some interesting remarks that are worth being shared with the interested professionals (audiologists, otologists, etc.).

First to mention is the typology of mechanical stimulation that is obtained via a piezoelectric modality. The bellow of both transducers is in fact made of several layers of crystals that are able to deliver energy when displaced, and to be displaced when reached by energy. Furthermore, this type of modality can be considered optimal for high-frequency stimulation with limited energy consumption. This latter factor explains why for the Esteem ${ }^{\circledR}$ there is no need to recharge the battery that, however, needs to be replaced after a certain time of use (around 5 years on average in our overall experience). In this regard, it has been noticed that the battery was more likely to be extinguished faster in case of a continuous $(24 / 24,7 / 7)$ use and in case of more advanced forms of SNHL.

The surgical procedure is somewhat demanding even for experienced otosurgeons who must undergo laboratory training before starting with clinical application. The complexity of the procedure is mostly related to a few, important steps that require the use of different types of cement, as well as to specific dexterity in working in very narrow spaces opened during the procedure, like for example when cementing the driver together with the precoated stapes head through the posterior tympanotomy. This is explaining why the surgical procedure can take long time especially with the first cases. In this regard, it is noteworthy to stress the importance of a bioengineer present in the operating theater, carrying out objective measurements by LDV during and at the end of surgery, thus offering confident and supporting data to the surgeon for a beneficial final functional outcome. As far as cement is concerned, two different types were used: a bio glass cement in small amounts for stapes pre-coating, incus neo-joint and stapes/driver fusion, and when larger amounts of cement were needed, as for stabilization of the transducers body within the mastoidectomy cavity, a hydroxyapatite compound was used.

Apart from the inescapable complications related to the laser resection of part of the incus long process and to the ablation of the chorda tympani nerve, no other intraoperative complications were recorded in our series. However, in less than $10 \%$ of the subjects, delayed, transient facial nerve palsy developed, with return to normality in all cases within the first month after onset.

The auditory outcome has been shown to be positive in over $80 \%$ of the subjects, although with variable degrees of improvement. In very few cases, it has been necessary to perform a revision surgery for debridement of newly formed fibrous tissue in the middle ear that was impeding the normal motility of the transducers. Another interesting finding relates to the observation of a striking decrease of the auditory threshold (as bone conduction) observed over time especially in those individuals who presented an advanced SNHL, being severe-to-profound, while not affecting the contralateral, non-implanted ear, so as to rule out to be dependent upon the causative factor. This new functional situation was in some cases still managed by setting the Esteem SP accordingly, while in a few subjects required the "transition" to cochlear implantation. 
As mentioned before, the Esteem ${ }^{\circledR}$ is not requiring a daily battery recharge, contrary to the other fully implantable device existing today (Carina ${ }^{\circledR}$ ). Therefore, the protocol entails a surgical substitution when the battery is going to extinguish (signaled by a double beep heard by the subject, a few weeks before end of the function). While the company mentioned a duration between 5 and 9 years, our personal experience showed a shorter duration, on average being 4-5 years, related mostly to the duration of use and to the severity of SNHL. In fact, most of the subjects were never switching off the device, as proof of achievement of a better quality of life in several daily moments (washing, bathing, sleeping, and performing physical activities). This important feature belonging to an invisible system has also been object of a previous report [7].

\section{Conclusions}

The fully implantable Esteem ${ }^{\circledR}$ active middle ear device has shown to provide a beneficial hearing gain in the majority of the implanted subjects. Our experience has shown that this may be achieved with very low morbidity as verified by the low incidence of complications. Other than for moderate-to-severe SNHL, the Esteem ${ }^{\circledR}$ may be indicated also for worse hearing threshold for which it can still provide a beneficial auditory and quality of life outcome. Despite its active mechanical role for eliciting cochlear stimulation, in certain candidates, it has been shown a progressive deterioration of the bone conduction threshold so as to limit the use of the implant and rather suggest cochlear implantation.

\section{Acknowledgements}

The authors wish to express their gratitude to Dr Kelly Brooks who helped with intra-operative measurements and patients' audiological fitting.

\section{Conflict of interest}

No conflict of interest to declare.

\section{Author details}

Maurizio Barbara* and Simonetta Monini

ENT Clinic, NESMOS Department, Sapienza University, Rome, Italy

*Address all correspondence to: maurizio.barbara@uniroma1.it

IntechOpen

(C) 2019 The Author(s). Licensee IntechOpen. This chapter is distributed under the terms of the Creative Commons Attribution License (http://creativecommons.org/licenses/ by/3.0), which permits unrestricted use, distribution, and reproduction in any medium, provided the original work is properly cited. (cc) BY 


\section{References}

[1] Kraus EM, Shohet JA, Catalano PJ. Envoy Esteem totally implantable hearing system: Phase 2 trial. I-year hearing results. Otolaryngology and Head and Neck Surgery. 2011;23:1-10

[2] Kraus EM, Shohet JA, Catalano PJ. Totally implantable hearing system: Five-year hearing results. Laryngoscope. 2018;128:210-216

[3] Barbara M, Biagini M, Monini S. The totally implantable middle ear device 'Esteem' for rehabilitation of severe sensorineural hearing loss. Acta OtoLaryngologica. 2011;131:399-404

[4] Seidman MD, Standring RT, Ahsan S, et al. Normative data of incus and stapes displacement during middle ear surgery using laser Doppler vibrometry. Otology \& Neurotology. 2013;34:1719-1724

[5] Monini S, Biagini M, Atturo F, Barbara M. Esteem middle ear device versus conventional hearing aids for rehabilitation of bilateral sensorineural hearing loss. European Archives of Oto-Rhino-Laryngology. 2013;270:2027-2033

[6] Shohet JA, Kraus EM, Catalano PJ. Profound high-frequency sensorineural hearing loss treatment with a totallyimplantable hearing system. Otology \& Neurotology. 2011;32:1428-1431

[7] Monini S, Bianchi A, Talamonti R, Atturo F, Filippi C, Barbara M. Patient satisfaction after auditory implant surgery: Ten-year experience from a single implanting unit center. Acta OtoLaryngologica. 2016;137:389-397

[8] Barbara M, Volpini L, Monini S. Delayed facial nerve palsy after surgery for the Esteem fully implantable middle ear hearing device. Acta OtoLaryngologica. 2014;134:429-432 
Section 3

\section{Cochlear Implants}





\title{
The Need to Increase Awareness and Access to Cochlear Implantation
}

\author{
Patrick S.C. D’Haese, Vincent Van Rompaey, Marc De Bodt \\ and Paul Van de Heyning
}

\begin{abstract}
Some degree of disabling hearing loss is present in 466 million people worldwide, representing $5 \%$ of the world's population and the majority of these are adults over 65 years old. Hearing loss is associated with increased risks of social isolation, depression, dementia, stroke, vision loss, diabetes and mortality. It is in the top five causes of years lived with disability in 2015, 2016 and 2017 for males and top 10 for females. Hearing aids are a suitable treatment for mild to moderate loses but for some they do not provide enough benefit. Cochlear implantation is a proven and effective treatment for bilateral severe to profound hearing loss, yet despite good funding in high income countries, the utilisation of CI is poor (less than $10 \%$ of suitable patients), especially in the older adult population who arguably need it most. Prevalence data shows that hearing loss increases with age, but the provision of implants in the over $65 \mathrm{~s}$ is even lower, despite there being no clinical barriers to older adults receiving a CI. Survey data shows that awareness activities are needed for both professionals and the general population to improve knowledge of what a $\mathrm{CI}$ is and how it can help.
\end{abstract}

Keywords: hearing loss, cochlear implant, health belief model, awareness

\section{Introduction}

Within Europe, we see an ageing population that is more active in their community and society than ever before. In order to sustain a high level of quality of life it is important for the European citizens to be able to communicate independently, remain active and maintain their autonomy. Untreated hearing loss very quickly leads to social isolation and depression. Patients speak of the impact of fragmented communication on their inability to participate in conversation leading to a diminishing circle of friends. Hearing loss also has the potential to restrict a person's independence, limiting their ability to take care of themselves or partners, accelerating their progression into facilitated living or social care. Whilst there are obvious benefits to living longer, such as opportunities to pursue a lengthier career, discover new hobbies, explore further education and spend quality time with family, there are also associated risks. Perhaps, when one considers these risks one thinks of increased frailty, or maybe cognitive decline, but what about the impact of hearing loss and its associated morbidities on quality of life? 
Some degree of disabling hearing loss is present in 466 million people worldwide, representing $5 \%$ of the world's population and the majority of these are adults over 65 years old (93\%) [1]. Our population is ageing and by $2025,20 \%$ of the population will be over 65 years. We know that the prevalence of hearing loss increases with age and roughly $30 \%$ of men and $20 \%$ of women in Europe are expected to have a hearing loss of $30 \mathrm{~dB}$ HL or more by age 70 years, and $55 \%$ of men and $45 \%$ of women by age 80 years [2]. The prevalence of child and adult hearing impairment is substantially higher in middle- and low-income countries than in high-income countries, demonstrating the global need for attention to hearing impairment [3].

Fortunately, treatment for hearing loss is more advanced than ever before. Hearing aids (externally worn amplification) and hearing implants (surgically implanted and replacing the function of the middle or inner ear) are widely available. Cochlear implants have been used successfully for over 30 years and are an effective treatment used for those who experience hearing loss too severe to benefit from a hearing aid [4]. The surgery required for a cochlear implant (CI) is largely considered routine with a low complication rate and benefits are large, with significant gains in quality of life [5]. Medical technologies such as cochlear implants can play an important role in reversing the impact of hearing loss on the health and wellbeing of the individual. In addition, these technologies will also help to reduce the impact on the economy and society. For example, in a study that looked at $93 \mathrm{CI}$ users, 6 years after they had been implanted, statistics demonstrated that the unemployment rate had dropped from 60 to $49 \%$. The same study also demonstrated the impact of hearing loss on personal income. One of the conclusions is that $31 \%$ of respondents had an increased income which was high enough after treatment to move income brackets [6]. It should be noted that the positive economic impact of treating hearing loss in a child continues up until old age. Several studies have demonstrated that children with hearing loss, which is not treated, are less likely to attend mainstream education. This will have an overall impact on their employment opportunities and earnings potential. Cochlear implants are largely funded by national health care or insurance systems in high income countries and in theory should be available to all who want one and meet the clinical criteria. Yet, at present, evidence suggests that utilisation amongst suitable adults is very low (less than $10 \%)$ and more work needs to be done to ensure patients have access to this technology.

\section{Global disease burden of hearing loss}

The first question to ask is how big a problem hearing loss represents globally? Global disease burden studies bring together all currently available epidemiological data on a disease to provide comparisons of death, prevalence and loss of health over time. In order to align health systems with the populations they serve, policymakers first need to understand the true origin and impact of their country's health challenges and how those challenges are shifting over time. This requires more than just estimating disease prevalence, such as the number of people with depression or diabetes in a population. Global disease burden research incorporates both the prevalence of a given disease or risk factor and the relative harm it causes. The tools allow decision-makers to compare the effects of different diseases, such as diabetes type 2 versus cancer, and then use that information to guide policy. Global burden of diseases, injuries, and risk factors studies provide a comprehensive assessment of prevalence, incidence, and years lived with disability (YLDs) for over 300 causes in 195 countries and territories. Health losses are expressed in terms of years lived with 
disability (YLD) and is calculated by multiplying the prevalence of the condition by a 'disability weight' which reflects the severity of the condition in comparison with other conditions. Globally, low back pain, migraine, age-related and other hearing loss, iron-deficiency anaemia, and major depressive disorder were the five leading causes of YLDs in 2015 and 2016. In the most recent report, age related hearing loss remains in the top five for males and top 10 for females [7-9].

Part of the reason hearing loss ranks highly in its disease burden is due to the prevalence of hearing loss in the population. The current World Health Organisation (WHO) 2018 figures report that as many as 466 million people have a disabling hearing loss (defined as a loss in the better ear of $>40 \mathrm{~dB}$ ), i.e. $5 \%$ of the world's population (Figure 1). This number is set to rise to possibly 630 million by 2030 as life expectancy increases and the population ages (Figure 2). As we age the sensory cells in our inner ear irreversibly degenerate resulting in age related hearing loss. This natural process can be accelerated by exposure to high levels noise during our lifetime and this is most likely to account for the difference in male and female prevalence rates due to the type of employment (Figure 2). Thus, we can see from the data in Figure 3 that the prevalence of hearing loss increases with age, with less than $2 \%$ of children likely to experience hearing loss but almost one in three adults experiencing a disabling hearing loss after the age of 65 years. This pattern holds true for all regions globally, regardless of income (Figure 3). An important factor to mention as well is prevention. There is a need to raise awareness on several preventable causes of hearing loss such as ototoxicity and noise induced hearing loss (recreational and industrial) but also to further stress the importance of aspects such as vaccination and hearing screening. The latter can be in neonates but also in adults where progressive hearing loss is a major issue [1].

The second factor in the assessment of global disease burden is the degree of disability caused by the sensory loss. The most obvious of these come from the loss of the ability to communicate effectively and can lead to social isolation and depression. This is especially apparent when the hearing loss is severe to profound. There is a body of evidence showing that those with a hearing impairment feel lonelier or more socially isolated, anxious or distressed than their normally hearing peers and this has an impact on measures of overall quality of life (for more details see Shield report section B [10]).

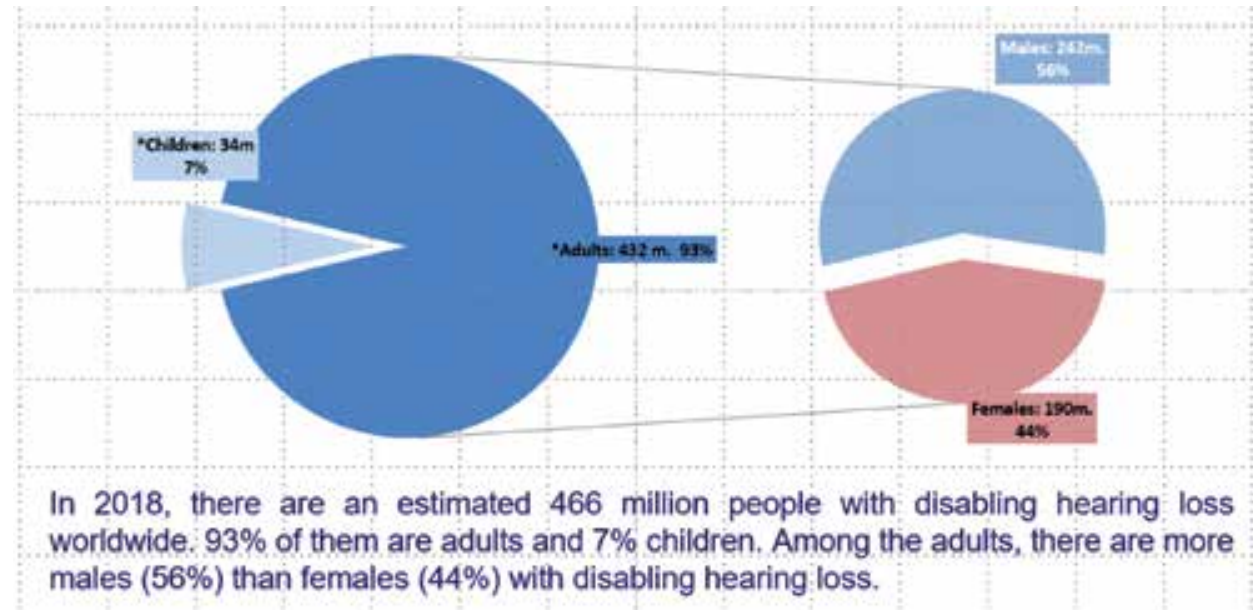

Figure 1.

The 2018 figures for disabling hearing loss are shown. The left-hand pie chart shows the proportion of adults and children with a disabling loss and the right-hand pie chart shows the split between male and female. 


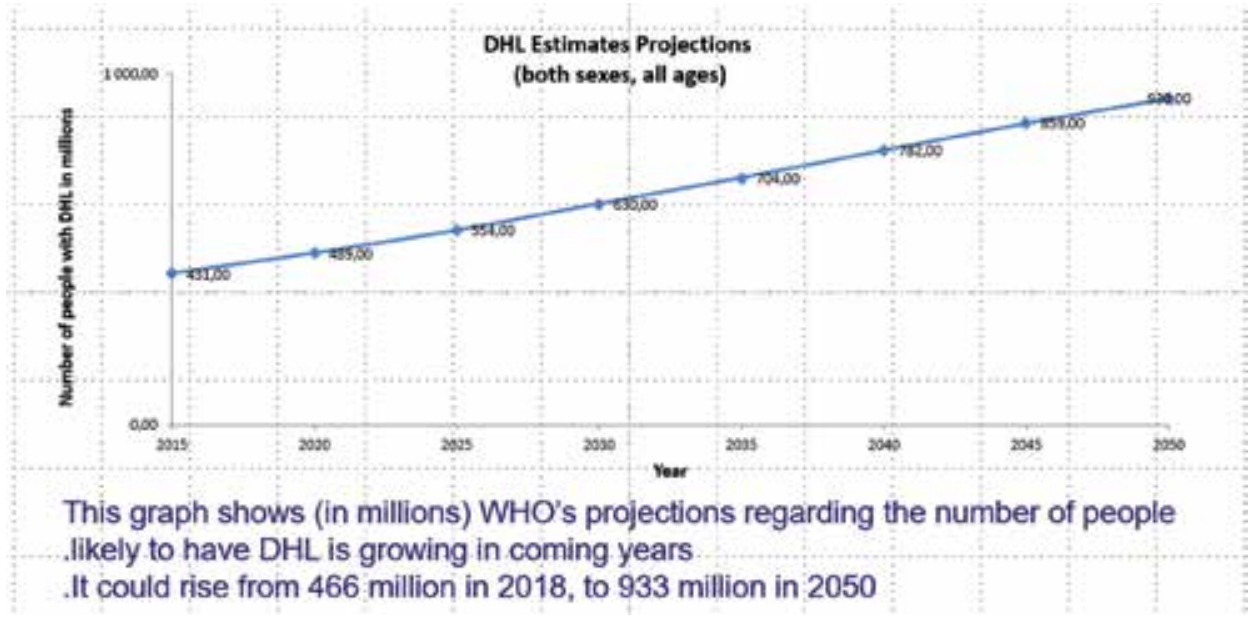

Figure 2.

This graph shows in millions the number of people with disabling hearing loss over time and the WHO's projections for the number of people likely to have a disabling hearing loss in the future.

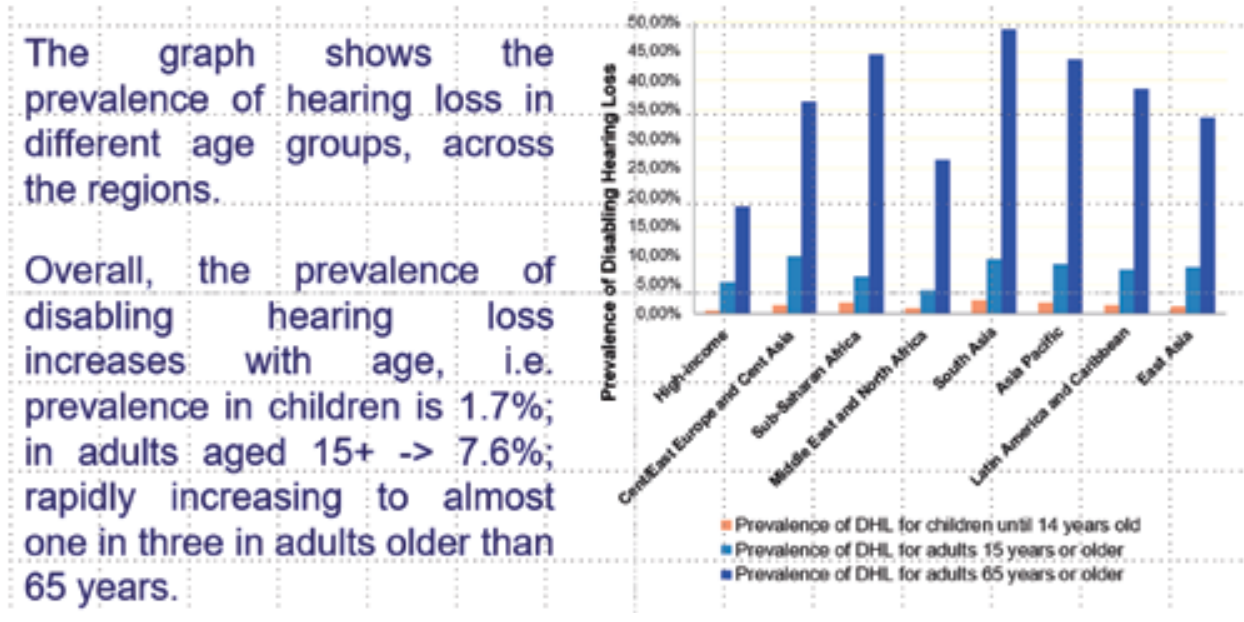

Figure 3.

The graph shows the prevalence of hearing loss in the different age groups across the regions. Overall the prevalence of disabling hearing loss increases with age to almost one in three adults after 65 years old.

People are less aware of the relationship between hearing loss and other aspects of overall health: these include the risks of more falls, an association with diabetes, stroke and sight loss [11-15]. For adults aged 70 and older, hearing loss is independently associated with hospitalisation and poorer self-reported health [16] There is also good evidence that an increased mortality rate is associated with hearing loss; this is most likely to be via three mediating variables: disability in walking, cognitive impairment and vision loss [17-19]. However, more recent data questions these results: Schubert et al. followed over 2400 participants in the US for up to 17 years, and found that although initially hearing loss was associated with mortality, when taking account of additional risk factors for cardiovascular and other diseases, the association between hearing loss and mortality was no longer significant [20]. They also found that participants who developed hearing impairment during the follow up period did not have an increased risk of mortality. Similarly, Amieva et al., in a 25 year longitudinal study in France, found no difference in risk of mortality between subjects with and without self-reported hearing loss [21]. 
The Need to Increase Awareness and Access to Cochlear Implantation

DOI: $h t t p: / / d x$.doi.org/10.5772/intechopen.86972

\begin{tabular}{lll}
\hline Archbold et al. [29] & UK (all hearing loss) & 30 billion pounds per year \\
\hline WHO [30] & Global (all hearing loss) & $\$ 700-800$ billion per year \\
\hline $\begin{array}{l}\text { Deloitte Access Economics } \\
{[31]}\end{array}$ & Australia (all hearing loss) & $\begin{array}{l}\text { 15 billion Australian dollars per } \\
\text { year }\end{array}$ \\
\hline Mohr et al. [32] & $\begin{array}{l}\text { USA (severe to profound hearing } \\
\text { loss) }\end{array}$ & \begin{tabular}{l} 
\$400,000 per person over lifetime \\
\hline
\end{tabular} \\
\hline
\end{tabular}

Table 1.

Examples of estimates of the costs of untreated hearing loss in high income countries [10].

Hearing loss has also been strongly linked to cognitive decline and dementia $[22,23]$. A hearing loss as little as a $25 \mathrm{~dB}$ was found to be equivalent to the cognitive reduction associated with an age difference of 6.8 years [24] People with mild hearing loss are twice as likely to develop dementia as those without hearing loss and five times more likely to develop it if they have a severe loss [25]. Hearing loss not only increases the risk of developing dementia but accelerates the rate of cognitive decline [26] The Lancet commission on Dementia Prevention, Intervention, and Care describes the new life-course model of dementia prevention incorporating nine modifiable risk factors and their potential effect in reducing an individuals' risk of dementia [27]. They were surprised to find that hearing loss was the largest contributor, almost doubling the risk. Overall, they concluded that the most promising intervention targets were increasing education in early life, increasing physical activity and social engagement, reducing smoking, treating hypertension, diabetes, and hearing impairment.

As well as having a significant impact on the individual in terms of a reduction in their quality of life, there is also a significant cost to society as a result of unmanaged hearing loss. A value can be calculated for lost productivity in terms of income and taxes. Employment rates for hearing impaired individuals are on average about $20 \%$ lower compared to those without hearing loss and those who are in work often take lower paid jobs, work part time and retire early [10, 28]. There is also a cost associated with increased pressure on health services, for example more visits to the general practitioner or from social services [29]. By combining these figures and including an amount for loss of quality of life, various estimates have been made for the costs associated with hearing loss of $25 \mathrm{~dB}$ or more in various high-income countries (Table 1). World-wide estimates are as high as 700-800 billion dollars per year globally, and ranges from around 476 to 581 billion euros in Europe. Restricting the calculations to hearing loss of $35 \mathrm{~dB}$ and above, the cost to the EU ranges from approximately 185-261 billion euros, and to the wider European community from approximately 216-303 billion euros, depending on whether or not the use of hearing aids is accounted for.

\section{Treatment options and their use}

Although hearing loss is not always preventable, it is treatable. The degree of loss can be described in four categories form mild to profound (Table 2) and for those with a mild to moderate loss, hearing aids are an effective intervention, as can be seen from the list of reported benefits described in Table 3. However, when these are no longer sufficient, most commonly in severe to profound hearing loss, $\mathrm{CI}$ is indicated.

A CI is a neural stimulator providing electrical stimulation directly to the hearing nerve and is different to a hearing aid, which amplifies sound and presents 


\begin{tabular}{|c|c|c|}
\hline $\begin{array}{l}\text { Degree of } \\
\text { hearing loss }\end{array}$ & $\begin{array}{l}\text { Quietest sound } \\
\text { heard }(\mathrm{dB} \mathrm{HL})\end{array}$ & Effects \\
\hline Mild & $25-39$ & Can sometimes make following speech difficult \\
\hline Moderate & $40-69$ & May have difficulty following speech without hearing aids \\
\hline Severe & $70-94$ & $\begin{array}{l}\text { Usually need to lipread or use sign language, even with } \\
\text { hearing aids }\end{array}$ \\
\hline Profound & $95 \mathrm{~dB}$ or greater & Usually need to lipread or use sign language \\
\hline
\end{tabular}

Table 2.

Action on hearing loss classifications of hearing loss (https://www.actiononhearingloss.org.uk).

Over $80 \%$ of hearing aid users report improvements in their overall quality of life.

Over $60 \%$ of hearing aid users in Europe and the USA report improvements in the ability to communicate effectively and over $50 \%$ in the ability to participate in group activities.

Hearing aid users in Eurotrak surveys report less physical and mental exhaustion, better sleep, less depression and better memory than non-users.

There is an association between hearing aid use and reduction in cognitive ability; recent research suggests that hearing aid use reduces the rate of cognitive decline.

There has been conflicting research evidence concerning the impact of hearing aids on depression but there is a suggestion that the use of hearing aids may reduce depressive symptoms.

Results of studies concerning the impact of hearing aids on loneliness and social isolation are inconclusive.

Hearing aid users earn significantly more than non-users, the differential between the two groups increasing with the severity of hearing loss.

Unemployment rates of non-users are approximately twice those of hearing aid users.

Table 3.

Proven benefits of wearing a hearing aid taken from a comprehensive analysis of the published data in the Shield report [10], addressing all the consequences of hearing loss listed above.

it through the damaged hearing system. It consists of an implanted device and externally worn audio processor (Figure 4). During surgery an electrode array is inserted into the cochlea which provides stimulation directly to the spiral ganglion cells. The external processor picks up the sound via a microphone and converts it into a series of coded signal which are transmitted via a RF link to a receiver placed into a shallow well in the mastoid bone, which is connected to the electrode array via the middle ear cavity (Figure 5). A CI is an effective treatment for profound hearing loss [33-35]. Adults, who have normal speech development as a child and have acquired their hearing loss in later life, can expect to be able to use the phone, interact in social groups in quiet and for some of the better performers, even hear effectively in noise and enjoy music. Some of the comments implant users have made post implantation include: going out for a meal is a pleasure again because I do not feel excluded from the conversation. I feel less isolated knowing I can hear the doorbell ring. I can relax when I watch TV instead of straining to hear and reading the subtitles. The results are not instant, and the first 3 months show the largest changes in what people can hear and understand, although improvement continues over the first year. However, despite the magnitude of the benefits and the significant impact of implantation on quality of life the take up of CI in adults is poor.

Utilisation rates for CI, in both adults and children with severe to profound loss, were reported in Sorkin and Buchman for Germany, Austria, Sweden, United Kingdom, Australia and the USA [36]. These were based on the known implanted population and prevalence data collected in the respective countries. Adult 


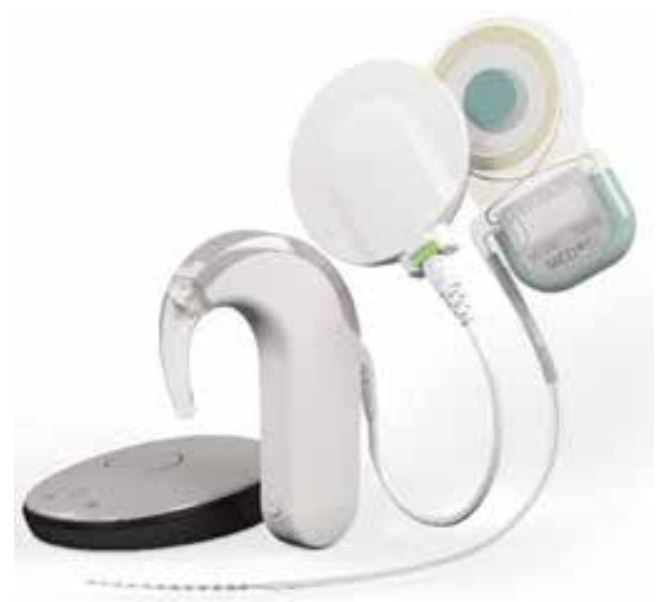

Figure 4.

Image of a cochlear implant showing the implanted and externally worn audio processor parts (behind-the-ear audio processor and single unit audio processor (courtesy of MED-EL GmbH).

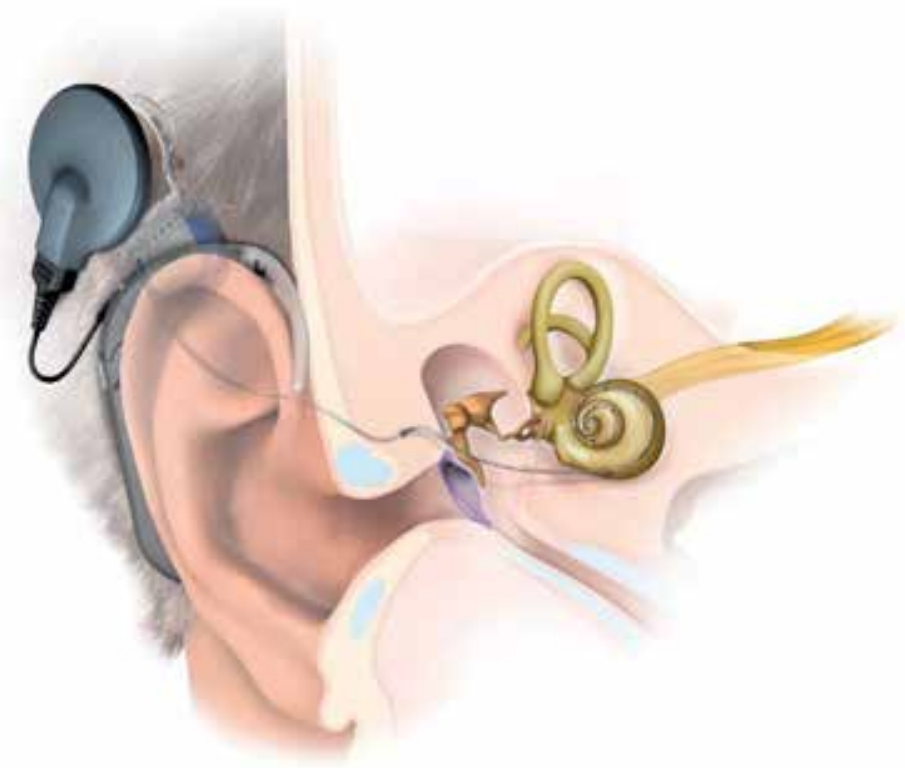

Figure 5.

Image showing the cochlear implant in situ. The external microphone picks up the sound, which is then transmitted wirelessly to the internally implanted parts (courtesy of MED-EL GmbH).

penetration was found to be less than $10 \%$, regardless of geographic region. This is surprising given that rates for adult hearing aid use in people with a severe or profound hearing loss are high, at an average of 63\% across Europe [10] and leads us to question why the uptake of $\mathrm{CI}$ in the adult population is so poor. Prevalence data has shown that hearing loss increases with age, but the provision of implants in the over $65 \mathrm{~s}$ is lower than in younger people, despite there being no clinical barriers to older adults receiving a CI [36-39]; for example in Australia, utilisation in the over $65 \mathrm{~s}$ is $0.3 \%$. The benefits to older adults of the CI are almost the same as the benefit to the younger person and the poor uptake of treatment might be due to the perception of hearing loss as a natural consequence of the ageing process by both patient 


\begin{tabular}{ll}
\hline Reasons for non-ownership & $\%$ \\
\hline Hear well enough in most situations & 64.3 \\
\hline Hearing loss not severe enough & 61.8 \\
\hline Uncomfortable & 56.0 \\
\hline They do not restore your hearing to normal & 54.2 \\
\hline They do not work well in noisy situations & 54.2 \\
\hline
\end{tabular}

Table 4.

Average percentages of respondents citing reasons for non-ownership of hearing aids.

and professional. This acts as an additional barrier to treatment for this group and it has been shown that people who regarded hearing loss as being inconsequential and accepted it passively, are less likely to seek treatment [40]. Paediatric use of CI does not follow the same pattern with much higher penetration rates in all regions (e.g. $97 \%$ in Australia, 50\% in the USA), highlighting the different approach to childhood hearing loss.

To seek treatment for a condition, individuals must perceive that they have a hearing loss which is severe enough to be perceived as a threat to their overall wellbeing [41]. They must also feel that there are more benefits than barriers to the treatment sought. Factors identified as acting as barriers to hearing rehabilitation in general include financial limitations, stigma of hearing devices, inconvenience, competing chronic health problems and unrealistic expectations [42] The Eurotrak survey data collected in 2015 illustrates this and found that the main reasons given for not wearing a hearing aid were that the hearing loss was not severe enough and that hearing aids were uncomfortable and embarrassing to wear (Table 4) [43].

There are a number of factors which may adversely affect the uptake of CI compared to hearing aids. Firstly, it is a more costly treatment with an adult surgery and follow up. However, CIs have been shown to be highly cost effective with low complication rates and in most developed economies, funding is provided either by a national health programs or employer based or private insurance schemes $[33,44]$. None the less, lack of funding has the potential to restrict the number of suitable adults who are implanted, particularly in undeveloped economies. However, the costs of not effectively treating hearing loss have been shown to be much greater and, as we have seen earlier, investment in treatment at an early stage may reduce the burden on health services of other diseases such as dementia. Poor awareness of the treatment amongst professionals and patients or even a negative perception of implants in the general population, due to the political issues around the impact implantation has on deaf culture and the deaf community is also a factor.

\section{Raising awareness}

One of the issues which may be inhibiting adults from coming forward to be assessed for a CI is lack of awareness of the treatment in both professionals and potential recipients. Good knowledge of the risks verses benefits is especially important for cochlear implantation as it requires an invasive surgical procedure to insert the electrode array into the cochlea, in contrast to a hearing aid which can be fitted at any time. It is therefore beholden to the professionals to provide accurate information to enable potential patients to balance the risks verses benefits and make an informed choice. Evidence has shown that uninformed professionals can act as an additional barrier to implantation and improved education 
of audiologists and ENT specialists can greatly improve referral rates [41, 45]. It would be reasonable to assume that professionals such as audiologists and otorhinolaryngologists were well informed about the benefits and limitations of a CI and were able to refer appropriately. However, very little published data exists and the most recent of these studies, Chundu and Buhagiar, indicate that less than half of the audiologists surveyed were confident that they knew when to refer a patient for a CI assessment [46].

Behaviour patterns are explained and modified by using different theories of learning use models. One of these models is the health belief model by Rosentstock. It provides a framework to describe how people can be influenced to change their health-related behaviour [47]. Within the model it is stated that there are three conditions required to change behaviour: (1) the existence of sufficient motivation (or health concern) to make health issues salient or relevant. (2) The belief that one is susceptible or vulnerable to a serious health problem or to the sequelae of that illness or condition (often termed 'perceived threat'). (3) The belief that following a particular health recommendation, at a subjectively acceptable cost, would be beneficial to reducing the perceived threat. Within this latter condition, cost refers to the perceived barriers that must be overcome in order to follow the health recommendation. This cost refers to, but is not restricted to, financial outlays. Applying this into the area of disabling hearing loss, we must consider that people will take action to seek treatment for disabling hearing loss if they view this disabling hearing loss as a serious issue, they feel sufficiently concerned about the severity of their disabling hearing loss, and that there are more benefits than barriers to amplification or an alternative way of stimulation/amplification [46].

More healthcare choices are driven by patients and the internet than ever before. If utilisation of cochlear implants is to increase, awareness of the impact of hearing loss and the knowledge of the benefits and limitations of cochlear implantation must be improved, and any misconceptions corrected. One way of doing this is to put in place an education campaign. The purpose of any such campaign would be to improve individuals' motivation to take action and seek treatment. In order to address this a large market research study, funded by MED-El GmbH, was undertaken to try to establish the levels of awareness in the older adult population. The aim of this study was to assess the factors that contribute to individuals' health motivation to take action and address their hearing loss. The older adult population (50-70 years old) was targeted as the prevalence of hearing loss is higher in this group. An online questionnaire with 13 closed set questions was completed by 500 subjects at home in Austria, Germany, France, Sweden and the United Kingdom (100 subjects in each country). The different questions were based around the health belief model, as shown in Figure 6 and looked at subject's perceived susceptibility to hearing loss, its severity (the perceived threat that hearing loss presents) and the barriers to action. Different questions were presented to assess subject's perceptions in three areas: (1) their susceptibility to disabling hearing loss, its importance to them and its impact (the threat); (2) the barriers that may prevent them from seeking appropriate treatment; (3) the actions that subjects currently take to address any hearing concerns.

Possible barriers to treatment of the disabling hearing loss were selected based on the personal experience of the authors. In addition, they were gained from focus groups and talking to existing CI users and clinicians involved in CI programmes. The following barriers were identified: the visibility of the device or cosmetics, convenience of use for all situations, including sports or going to bed, and management of the device. To assess the perception of both implants and hearing aids, as a hearing aid is the alternative treatment option, questions were designed. Barriers such as fear of surgery, losing residual hearing, and identity considerations were 


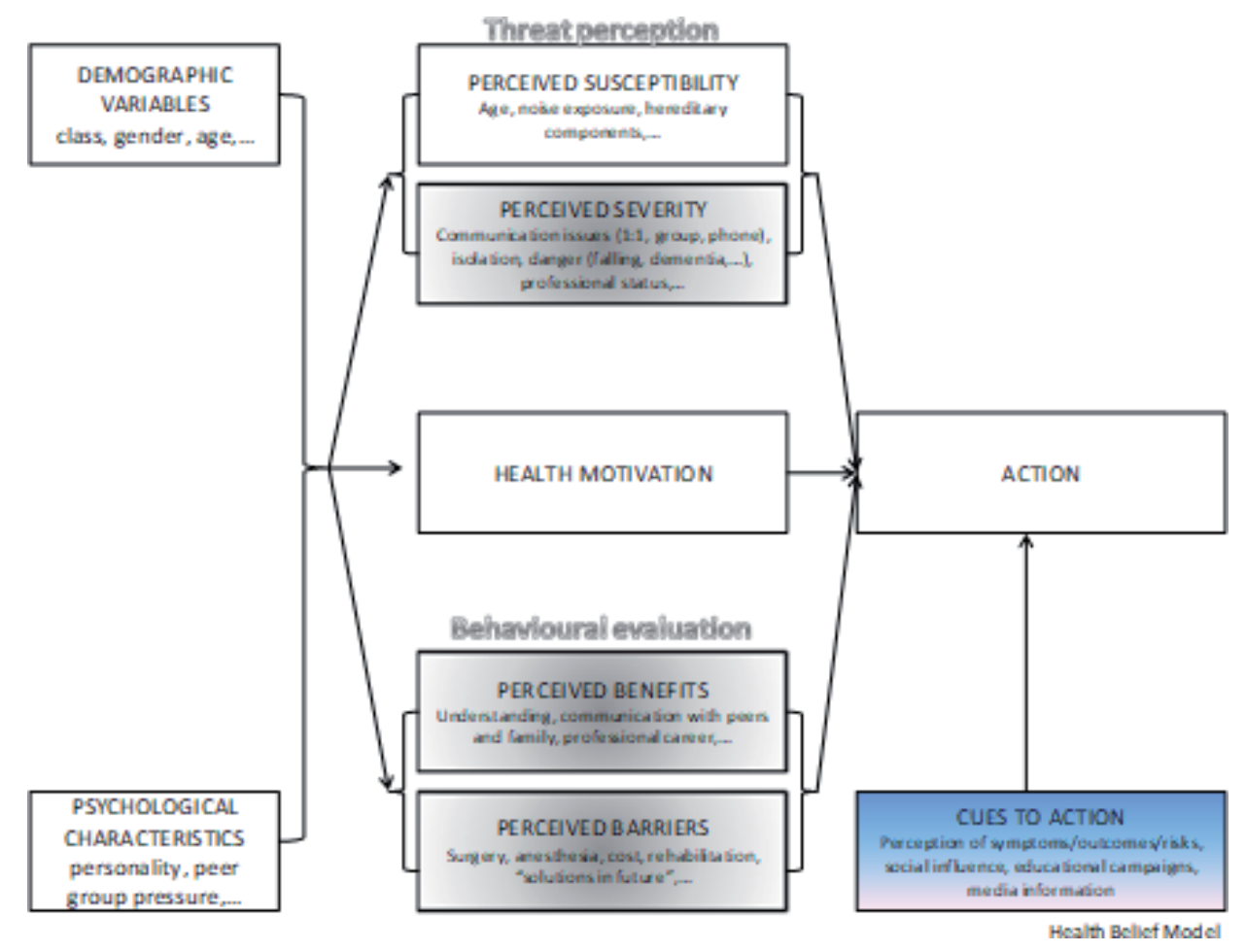

Figure 6.

Flow diagram describing the different factors from the health believe model which stimulate individuals to take action to address disabling hearing loss.

not considered as they were not identified by the focus groups at the time. However, these are also key areas for potential candidates to consider, even before referral to a specialist CI centre takes place. Questions were asked to the respondents whether they thought there was any difference between a hearing aid and a hearing implant and whether there was any difference in hearing sensitivity between hearing aids and implants. The underlying concept was to assess if a potential lack of perceived benefit or differences between implants compared to hearing aids was acting as a potential barrier to implantation.

In the resulting data, good hearing was regarded as being important in all countries and when motivated to do so, people sought information from medical professionals, mostly GPs and ENT specialists, about their hearing problems [48]. There was agreement that the main sign of hearing loss was turning up the TV or radio. This is a useful self-assessment measure which can prompt individuals to seek help. In more than the majority of the participating countries, hearing aids were thought to be not particularly visible, not require much maintenance nor servicing or a hindrance whilst doing sport. The majority of participants knew that they must be removed before bed. Perceptions of hearing implants were that they were permanently fitted, not externally visible and do not need to be removed before bed. This is a persistent and significant misconception and is discussed further in the next paragraph, where attitudes of professionals are also considered. In general, subjects knew that there was a difference between hearing aids and hearing implants (range of agreement 72-40\%). When asked if there was no qualitative difference (in terms of hearing sensitivity) between them, median scores tended towards disagree, this means that a subjective difference is also perceived. However it remains unclear whether the respondents understood what the differences were. 
Data has also been published relating to awareness of $\mathrm{CI}$ in doctors with an ear nose and throat (ENT) specialist training [49]. A random sample of 240 doctors were surveyed and their referral patterns, attitudes and beliefs towards CIs recorded. The results showed that they shared many common beliefs about hearing loss, hearing aids, and CIs, although some national variation in opinion was present. Most were aware that hearing implants and hearing aids were different, but nearly all would recommend a hearing aid over an implant if both were suitable. However, some doctors surprisingly agreed that there was no difference between hearing aids and hearing implants in terms of hearing sensitivity (5-36\%) and we would also have expected a strong disagree from well informed respondents to that statement. There was also some confusion over the need to still wear and maintain an external speech processor with a CI. Responses to the statements covering the types of issues which may come up in an initial consultation with a patient who is considering a CI were not as clear as could be expected, with Likert values falling within the middle of the range for most questions. Implants were still regarded by some as not externally visible and permanently fitted, without the need to remove them at night. This indicates that even in the professional group, not all were aware of the basic structure of a cochlear implant with a concealed implanted part and an external speech processor, which is worn much like a hearing aid. Without the external speech processor, the $\mathrm{CI}$ does not function and providing reassurance to patients that they can remove the external part of the CI at any time and return to their non-hearing state could be a key factor in the decision-making process. Conversely, misleading patients that a CI provides an invisible hearing solution, when currently it does not, can also be a hindrance. When initial discussions about hearing implants are taking place, the realisation by patients that the external parts of a hearing implant are not very different practically from a hearing aid may deter patients from pursuing a treatment option which they thought would be invisible.

Personal experience has shown that many potential CI recipients are not aware of the significant hearing benefits of a CI compared to a hearing aid. Potential implant recipients need to be able to make an informed choice about going forward for assessment. Individuals who would be suitable candidates are often resistant to considering an elective surgical procedure to address their hearing loss and accurate information on the risks and benefits needs to be available. The surveys indicated that in both the professional group and the general population more work needs to be done to improve awareness of the substantial benefits of CI and knowledge of the basics of the technology. The study results reinforced the importance of the internet in accessing information about health and hearing loss in the general population, but interactive new media were low down the list of preferred means of keeping abreast of medial issues for professionals in all countries. Whilst an online awareness campaign could be a cost-effective way of improving awareness of CI, professionals tended to rely on conferences and conversations with colleagues to keep up to date.

An awareness campaign was funded MED-EL GmbH which placed a series of banners in online newspapers and health magazines. If the reader clicked on the advert they were taken to the MED-El GmbH web site. This was a very broad approach with the objective to target a wide range of individuals, both professional and the general public. The campaign was run over a 2 week to 3 month period depending on the publication chosen. Click rates for the adverts were recorded and were at a median of around $0.2 \%$, although some outliers such as had considerably more traffic of 3-5\% for health publications such as Audiology World News. Although an average click rate of less than $1 \%$ seems very low, comparisons to industry data show that this is within the expected range for this type of advertising in the general population. It is reasonable to assume that the medical publications 
had a readership that were already interested in health topics and were thus more likely to click on the advert. This approach seemed promising, however, follow up awareness data collected from another random sample showed that there was little change in the attitudes of either the professional of general groups. Future campaigns should be focused on those who have already been identified as having a hearing loss but what form it should take or how this group could be accessed directly is less clear. Should the patients themselves be made aware of CI as a treatment option or should audiologists and ENT professionals be the focus? The pathway to improving awareness of $\mathrm{CI}$ is unclear, but it is certain that better management of hearing loss will be essential for the future in our ageing population.

\section{Conclusions}

- The prevalence of hearing loss is high with around 5\% of the world's population effected and this number is set to rise as the population ages.

- The burden of hearing loss as a disease is also high with hearing loss in the top five causes of years lived with disability in 2015, 2016 and 2017 for males and top 10 for females along with low back pain, migraine, iron-deficiency anaemia, and major depressive disorder.

- Cochlear implantation is a proven and effective treatment for bilateral severe to profound hearing loss, yet the utilisation of CI is poor (less than $10 \%$ of suitable patients), especially in the older adult population who arguably need it most.

- Survey data shows that awareness activities are needed for both professionals and the general population to improve knowledge of what a $\mathrm{CI}$ is and how it can help.

- Online activities are a cost-effective approach to improve awareness in the general public. However, professionals still get their information from conferences, colleagues and medical books and journals.

\section{Conflict of interest}

Patrick D'Haese is an employee of MED-EL GmbH, Innsbruck, Austria. Prof. Van de Heyning receives grants to the institution from MED-EL and Cochlear. 
The Need to Increase Awareness and Access to Cochlear Implantation DOI: $h t t p: / / d x$.doi.org/10.5772/intechopen.86972

\section{Author details}

Patrick S.C. D’Haese ${ }^{1 *}$, Vincent Van Rompaey ${ }^{2}$, Marc De Bodt ${ }^{2}$ and Paul Van de Heyning ${ }^{2}$

1 MED-EL, Innsbruck, Austria

2 Antwerp University Hospital, University of Antwerp, Edegem, Belgium

*Address all correspondence to: patrick.dhaese@medel.com

\section{IntechOpen}

(C) 2019 The Author(s). Licensee IntechOpen. This chapter is distributed under the terms of the Creative Commons Attribution License (http://creativecommons.org/licenses/ by/3.0), which permits unrestricted use, distribution, and reproduction in any medium, provided the original work is properly cited. (cc) BY 


\section{References}

[1] World Health Organisation. Global Estimates on Prevalence of Hearing Loss. 2018. Available from: https://www. who.int/pbd/deafness/estimates/en/ [Accessed: 28 March 2019]

[2] Roth TN, Hanebuth D, Probst R. Prevalence of age-related hearing loss in Europe: A review. European Archives of Oto-Rhino-Laryngology. 2011;268(8):1101-1107

[3] Stevens G, Flaxman S, Brunskill E, Mascarenhas M, Mathers CD, Finucane M. Global burden of disease hearing loss expert group. Global and regional hearing impairment prevalence: An analysis of 42 studies in 29 countries. European Journal of Public Health. 2013;23(1):146-152. DOI: 10.1093/ eurpub/ckr176

[4] Bond M, Mealing S, Anderson R, Elston J, Weiner G, Taylor R, et al. The effectiveness and cost-effectiveness of cochlear implants for severe to profound deafness in children and adults: A systematic review and economic model. Health Technology Assessment. 2009;13(44):1-330

[5] Lenarz T, Muller L, CzerniejewskaWolska H, Vallés Varela H, Orús Dotú C, Durko M, et al. Patient-related benefits for adults with cochlear implantation: A multicultural longitudinal observational Study. Audiology \& Neuro-Otology. 2017;22(2):61-73. DOI: $10.1159 / 000477533$

[6] Lavis JN, Posada FB, Haines A, Osei E. Bending the spend: Expanding access to hearing technology to improve health, wellbeing and save public money. Lancet. 2004;364:10

[7] GBD. Disease and injury incidence and prevalence collaborators. Global, regional, and national incidence, prevalence, and years lived with disability for 310 diseases and injuries,
1990-2015: A systematic analysis for the global burden of disease study 2015. Lancet. 2015, 2016;388(10053):

1545-1602. DOI: 10.1016/S01406736(16)31678-6. Erratum in: Lancet. 2017;389(10064):e1

[8] GBD. Disease and injury incidence and prevalence collaborators. Global, regional, and national incidence, prevalence, and years lived with disability for 328 diseases and injuries for 195 countries, 1990-2016: A systematic analysis for the global burden of disease study 2016. Lancet. 2017;390(10100):1211-1259.

DOI: 10.1016/S0140-6736(17)32154-2. Erratum in: Lancet.

2017;390(10106):e38

[9] GBD. Disease and injury incidence and prevalence collaborators. Global, regional, and national incidence, prevalence, and years lived with disability for 354 diseases and injuries for 195 countries and territories, 1990-2017: A systematic analysis for the global burden of disease study 2017. Lancet. 2018;392(10159):1789-1858. DOI: 10.1016/S0140-6736(18)32279-7

[10] Shield B. Hearing Loss Numbers and Costs-Evaluation of the Social and Economic Costs of Hearing Impairment. 2018. Available from: https://www.hear-it.org [Accessed: 28 March 2019]

[11] Lin FR, Ferrucci L. Hearing loss and falls among older adults in the United States. Archives of Internal Medicine. 2012;172(4):369-371. DOI: 10.1001/ archinternmed.2011.728

[12] Oh IH, Lee JH, Park DC, Kim M, Chung JH, Kim SH, et al. Hearing loss as a function of aging and diabetes mellitus: A cross sectional study. PLoS One. 2014;9(12):e116161. DOI: 10.1371/ journal.pone.0116161 
[13] Gopinath B, Schneider J, Rochtchina E, Leeder SR, Mitchell P. Association between age-related hearing loss and stroke in an older population. Stroke. 2009;40(4):1496-1498. DOI: 10.1161/ STROKEAHA.108.535682

[14] Chia EM, Mitchell P, Rochtchina E, Foran S, Golding M, Wang JJ. Association between vision and hearing impairments and their combined effects on quality of life. Archives of Ophthalmology. 2006;124(10):1465-1470

[15] Mitchell P, Gopinath B, McMahon CM, Rochtchina E, Wang JJ, Boyages SC, et al. Relationship of type 2 diabetes to the prevalence, incidence and progression of age-related hearing loss. Diabetic Medicine. 2009;26(5):483-488. DOI: $10.1111 / j .1464-5491.2009 .02710 . x$

[16] Genther DJ, Betz J, Pratt S, Martin KR, Harris TB, Satterfield S, et al. Association between hearing impairment and risk of hospitalization in older adults. Journal of the American Geriatrics Society. 2015;63(6):11461152. DOI: $10.1111 /$ jgs.13456

[17] Genther DJ, Betz J, Pratt S, Kritchevsky SB, Martin KR, Harris TB, et al. Association of hearing impairment and mortality in older adults. The Journals of Gerontology. Series A, Biological Sciences and Medical Sciences. 2015;70(1):85-90. DOI: 10.1093/gerona/glu094

[18] Karpa MJ, Gopinath B, Beath K, Rochtchina E, Cumming RG, Wang JJ, et al. Associations between hearing impairment and mortality risk in older persons: The blue mountains hearing study. Annals of Epidemiology. 2010;20(6):452-459. DOI: 10.1016/j. annepidem.2010.03.011

[19] Gopinath B, Schneider J, McMahon CM, Burlutsky G, Leeder SR, Mitchell P. Dual sensory impairment in older adults increases the risk of mortality:
A population-based study. PLoS One. 2013;8(3):e55054. DOI: 10.1371/journal. pone. 0055054

[20] Schubert CR, Fischer ME, Pinto AA, Klein BEK, Klein R, Tweed TS, et al. Sensory impairments and risk of mortality in older adults. The Journals of Gerontology. Series A, Biological Sciences and Medical Sciences. 2017;72(5):710-715. DOI: 10.1093/ gerona/glw036

[21] Amieva H, Ouvrard C, Meillon C, Rullier L, Dartigues JF. Death, depression, disability, and dementia associated with self-reported hearing problems: A 25-year study. The Journals of Gerontology. Series A, Biological Sciences and Medical Sciences. 2018;73(10):1383-1389. DOI: $10.1093 /$ gerona/glx 250

[22] Deal JA, Betz J, Yaffe K, Harris T, Purchase-Helzner E, Satterfield S, et al. Hearing impairment and incident dementia and cognitive decline in older adults: The Health ABC Study. The Journals of Gerontology. Series A, Biological Sciences and Medical Sciences. 2017;72(5):703-709. DOI: 10.1093/gerona/glw069

[23] Lin FR, Ferrucci L, An Y, Goh JO, Doshi J, Metter EJ, et al. Association of hearing impairment with brain volume changes in older adults. NeuroImage. 2014;90:84-92. DOI: 10.1016/j. neuroimage.2013.12.059

[24] Lin FR, Ferrucci L, Metter EJ, An Y, Zonderman AB, Resnick SM. Hearing loss and cognition in the baltimore longitudinal study of aging. Neuropsychology. 2011;25(6):763-770. DOI: $10.1037 / \mathrm{a} 0024238$

[25] Lin FR, Metter EJ, O'Brien RJ, Resnick SM, Zonderman AB, Ferrucci L. Hearing loss and incident dementia. Archives of Neurology. 2011;68(2):214-220. DOI: $10.1001 /$ archneurol.2010.362 
[26] Gurgel RK, Ward PD, Schwartz S, Norton MC, Foster NL, Tschanz JT. Relationship of hearing loss and dementia: A prospective, populationbased study. Otology \& Neurotology. 2014;35(5):775-781. DOI: 10.1097/ MAO.0000000000000313

[27] Orgeta V, Mukadam N, Sommerlad A, Livingston $\mathrm{G}$. The lancet commission on dementia prevention, intervention, and care: A call for action. Irish Journal of Psychological Medicine. Jun 2019;36(2):85-88. DOI: 10.1017/ ipm.2018.4

[28] Kochkin S. MarkeTrak VIII: The efficacy of hearing aids in achieving compensation equity in the workplace. The Hearing Journal. 2010;63(10):19-28

[29] Archbold S, Lamb B, O’Neill C, Atkins J. The Real Cost of Adult Hearing Loss. 2014. Available from: https:// www.earfoundation.org.uk/research/ adult-strategy-reports/the-real-cost-ofadult-hearing-loss-2014 [Accessed: 28 March 2019]

[30] World Health Organization. Global Costs of Unaddressed Hearing Loss and Cost-Effectiveness of Interventions: A WHO Report. Geneva: World Health Organisation. 2017. Available from: http://www.who.int/iris/ handle/10665/254659 [Accessed: 28 March 2019]

[31] Deloitte Access Economics. The Social and Economic Cost of Hearing Loss in Australia. Report Prepared for Hearing Care Industry Association (HCIA). 2017. Available from: www. hcia.com.au [Accessed: 28 March 2019]

[32] Mohr P, Feldman J, Dunbar J, McConkey-Robbins A, Niparko J, Rittenhouse $\mathrm{R}$, et al. The societal costs of severe to profound hearing loss in the United States. International Journal of Technology Assessment in Health Care. 2000;16(4):1120-1135
[33] Bond M, Elston J, Mealing S, Anderson R, Weiner G, Taylor R, et al. Systematic reviews of the effectiveness and cost-effectiveness of multichannel unilateral cochlear implants for adults. Clinical Otolaryngology. 2010;35(2):87-96

[34] Foteff C, Kennedy S, Milton AH, Deger M, Payk F, Sanderson G. Cost-utility analysis of cochlear implantation in Australian adults. Otology \& Neurotology. 2016;37(5):454-461. DOI: $10.1097 /$ MAO.0000000000000999

[35] Smulders YE, van Zon A, Stegeman I, van Zanten GA, Rinia AB, Stokroos RJ, et al. Cost-utility of bilateral versus unilateral cochlear implantation in adults: A randomized controlled trial. Otology \& Neurotology. 2016;37(1):38-45. DOI: $10.1097 /$ MAO.0000000000000901

[36] Sorkin DL, Buchman CA. Cochlear implant access in six developed countries. Otology \& Neurotology. 2016;37(2):e161-e164

[37] Chen DS, Clarrett DM, Li L, Bowditch SP, Niparko JK, Lin FR. Cochlear implantation in older adults: Long-term analysis of complications and device survival in a consecutive series. Otology \& Neurotology. 2013;34:1272-1277

[38] Davis A. Hearing in Adults: The Prevalence and Distribution of Hearing Impairment and Reported Hearing Disability in the MRC Institute of Hearing Research's National Study of Hearing. London: Whurr Publishers; 1995

[39] Zwolan TA, Henion K, Segel P, Runge C. The role of age on cochlear implant performance, use, and health utility: A multicenter clinical trial. Otology \& Neurotology. 2014;35(9):1560-1568 
[40] Van den Brink RH, Wit HP, Kempen

GI, van Heuvelen MJ. Attitude and help-seeking for hearing impairment. British Journal of Audiology. 1996;30(5):313-324

[41] Meyer C, Hickson L. What factors influence help-seeking for hearing impairment and hearing aid adoption in older adults? International Journal of Audiology. 2012;51(2):66-74

[42] Barnett M, Hixon B, Okwiri N, Irungu C, Ayugi J, Thompson R, et al. Factors involved in access and utilization of adult hearing healthcare: A systematic review. The Laryngoscope. 2017;127(5):1187-1194. DOI: 10.1002/ lary. 26234

[43] European Hearing Instrument Manufacturers Association (EHIMA). Euro Trak Surveys. 2015. Available from: www.ehima.com [Accessed: 28 March 2019]

[44] Vickers D, Kitterick P, Verschuur C, Leal C, Jenkinson L, Vickers F, et al. Issues in cochlear implant candidacy. Cochlear Implants International. 2016;17(Suppl 1):1-2. DOI:

10.1080/14670100.2016.1163104

[45] Raine C. Cochlear implants in the United Kingdom: Awareness and utilization. Cochlear Implants International. 2013;14(Suppl 1):S32-S37

[46] Chundu S, Buhagiar R.

Audiologists' knowledge of cochlear implants and their related referrals to the cochlear implant Centre: Pilot study findings from UK. Cochlear Implants International. 2013;14(4):213-224. DOI: 10.1179/1754762812Y.0000000025

[47] Rosenstock IM, Strecher VJ, Becker $\mathrm{MH}$. Social learning theory and the health belief model. Health Education Quarterly. 1988;15(2):175-183

[48] D’Haese PSC, De Bodt M, Van Rompaey V, Van de Heyning P.
Awareness of hearing loss in older adults: Results of a survey conducted in 500 subjects across 5 European countries as a basis for an online awareness campaign. Inquiry. 2018;55:46958018759421. DOI: $10.1177 / 0046958018759421$

[49] D’Haese PSC, Van Rompaey V, De Bodt M, Van de Heyning P. The knowledge and beliefs regarding practical aspects of cochlear implants: A study of otorhinolaryngologists in a secondary setting in a multicountry study. Cochlear Implants International. 2018;19(1):14-21. DOI: 10.1080/14670100.2017.1385141 



\title{
Advances in Surgical and Anesthetic Techniques for Cochlear Implantation
}

\author{
Yasser A. Fouad
}

\begin{abstract}
Cochlear implantation (CI) is usually performed under general anesthesia using the classic surgical approach, the mastoidectomy posterior tympanotomy approach (MPTA), which was originally described by William House in 1961. Many alternative surgical approaches have been described for CI. Robotic image-guided cochlear implantation has also been described as a new advance in CI. Also, in some situations, CI can be performed under conscious sedation with local anesthesia (CS-LA) instead of general anesthesia (GA). With the ongoing advance in CI devices and surgical techniques, CI surgery nowadays could preserve hearing in ears with preoperative residual hearing. This chapter describes different approaches and techniques in CI surgery, whether classic or alternative technique, with special attention to advantages and disadvantages of each approach or technique. Also this chapter describes, in surgical points of view, the anesthetic techniques in CI, whether GA or CS-LA, with focus on indications, advantages, and disadvantages of CS-LA in CI.
\end{abstract}

Keywords: cochlear implantation, mastoidectomy posterior tympanotomy approach, alternative approaches, round window, conscious sedation with local anesthesia, general anesthesia, receiver/stimulator

\section{Introduction}

Cochlear implantation (CI) is the surgical implantation of an electrical device that can directly stimulate the auditory nerve through bypassing a nonfunctional inner ear. Through this device, speech and other sounds can be heard by severe to profound deaf people [1].

The first "true" CI was performed by William House and John Doyle on January 9, 1961; the surgery was performed through postauricular incision using mastoidectomy posterior tympanotomy approach (MPTA) to the middle ear. The electrode array was inserted then, after exposure of the round window (RW) membrane, into the scala tympani [2].

Interestingly, the surgical approach used and described by William house in 1961 became the classic or the standard approach for CI; for more than half a century, there was no major advancement or change in the surgical approach. However, there were different alternative approaches and some technical advancements, each of them having relative advantages and disadvantages. 


\section{Surgical technique of "classic" cochlear implantation}

The surgical technique of classic CI was described in detail by House [3]. The basic surgical steps are the following:

\subsection{Skin incision}

Postauricular incision is the originally described incision for CI, and also it is the most common used incision for CI [4] (Figure 1).

\subsection{Elevation of periosteal flaps}

A "U"-shaped anterior-based periosteal flap or Palva flap (Figure 2) is performed to expose both the mastoid bone and the planed site for drilling a well "seat or bed" for the receiver/stimulator (RS).

\subsection{Mastoidectomy posterior tympanotomy approach}

MPTA is performed using both the surgical microscope and otologic drill. Widening of the posterior tympanotomy in an inferior direction with removal of excess bone in front of the facial nerve is an essential step for good exposure of RW niche and membrane, taking care that RW membrane may be obscured by a false membrane (false RW membrane) that should be removed first by sharp instrument (Figure 3).

\subsection{Drilling a bony well for the RS}

In classic $\mathrm{CI}$, fixation of the device is achieved mainly by drilling a custom-fit bony well "seat or bed" for accommodation of the thick part, the titanium case, of the RS of the selected implant. This well must be designed with the same configuration of the RS and should be deep enough so that the package rests in the well stably without the possibility of sliding or rocking and without protrusion outside the skull as a swelling [5].

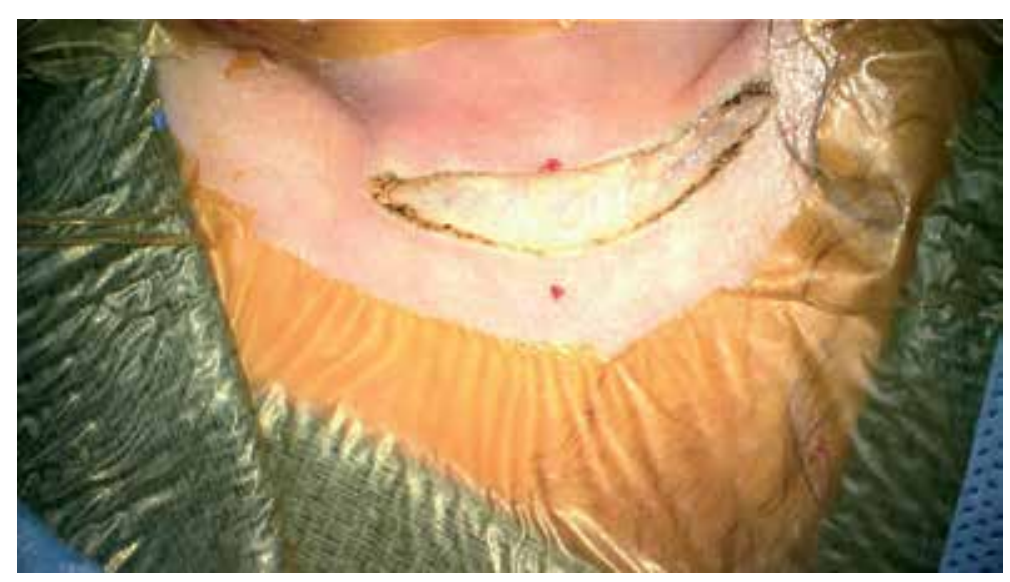

Figure 1.

Minimal access postauricular incision. 


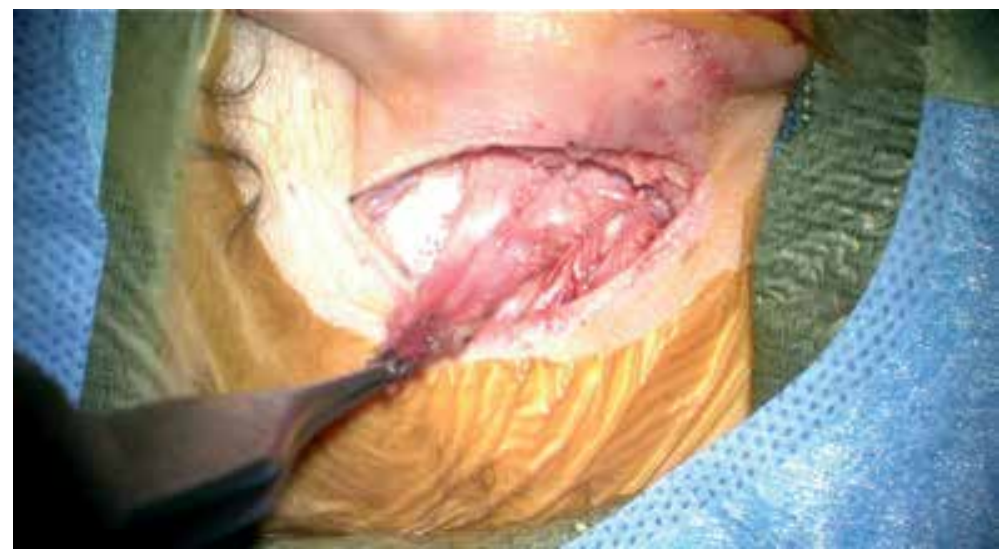

Figure 2.

Palva flap in CI.

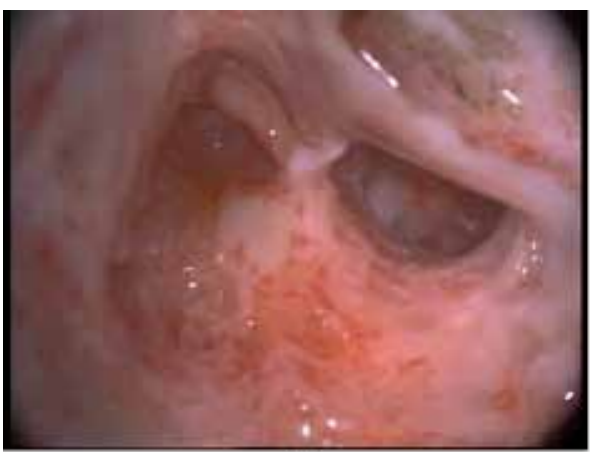

(a)

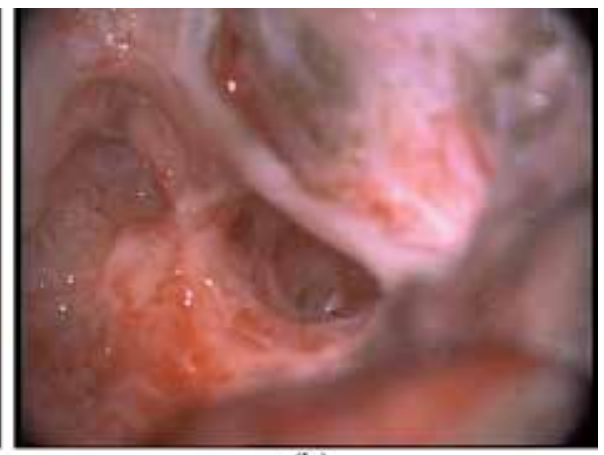

(b)

Figure 3.

(a) Classic MPTA showing good visualization of the RW niche and false membrane that cover true RW membrane. (b) After removal of the false membrane, RW membrane is now well visualized.

\subsection{Cochleostomy}

The RW niche is lowered down by drilling the tegmen and pillars of the RW till good exposure of the RW membrane is achieved, and then cochleostomy is performed. In classic CI, cochleostomy is drilled as a separate opening inferior and slightly anterior to the RW membrane [6].

\subsection{Electrode insertion}

The device is brought up to the surgical field, and then the electrode is inserted into the cochlea either by using the fine-tipped micro forceps or by using the specific instruments manufactured for insertion of the selected electrode type.

\subsection{Confirm device fixation}

The RS should be positioned in its drilled well "Seat or bed." Its stability in the well should be ensured. Sewing the periosteum together over the implant is also important for further stabilization [7].

The distal end of the electrode array should be secured and fixed. This is performed routinely by sealing off the cochleostomy site through harvesting a small 
piece of fascia or pericranium and then applying it around the electrode array at the cochleostomy site. This sealing also prevents transmission of infection from the middle ear into the cochlea [6]. Also the electrode array is further stabilized by placing a loop of electrode cable against the tegmen mastoideum [8].

\subsection{Intraoperative monitoring}

Intraoperative device monitoring is performed to confirm both electrical output of the device and electrical response of the patient at the same time. Intraoperative monitoring also provides objective data that can be used as a starting point for behavioral testing "psychophysics" [9].

First impedance telemetry, which confirms the integrity of the electrodes, is performed to all electrodes, and then the neural response of the patient can be tested by either measuring the electrical stapedial reflex thresholds (ESRT) or by measuring electrical compound action potential (ECAP), or neural response telemetry (NRT), which confirms stimulation of the auditory nerve. These electrical tests are essential to confirm the success of surgery; however, they are not a reliable predictor of postoperative performance [10].

\subsection{Wound closure}

The wound is closed in three layers: the periosteum, the subcutaneous layer, and the skin. Usually the dressing and pressure bandage are kept for 24 hours to reduce the possibility of a development of seroma or hematoma, then the wound is inspected, and another dressing is applied for another 5 days [5].

\section{Advances of the surgical technique}

Surgical technique of cochlear implantation was described in detail by House [3]; this description remains the classic or the standard surgical technique for cochlear implantation. Up till the time of writing of these words, there is no significant change in the basic surgical principles of the classic or standard CI. However, some surgical modifications and technical innovations were advanced and advocated by some surgeons. The most important surgical advances on the classic CI, according to our point of view, are listed in this section and sorted according to the consequence of surgical steps of CI.

\subsection{Skin incision}

The first described incision for CI was small postauricular C-shaped incision as the device has a single channel and is small in size, and then after inventing the multichannel devices, which had larger RS, larger postauricular C-shaped incisions or interior-based U-shaped incision were used. Due to the drawback of these large incisions on the blood supply with high incidence of flap necrosis, postauricular incision became the standard again and remained the most commonly used incision [4]. It was first long with an upward extension "inverted J-shaped incision" and then gradually become shortened by time. Nowadays many CI centers use the minimal access postauricular incision (Figure 1), which is $3-4 \mathrm{~cm}$ in length and $1 \mathrm{~cm}$ behind the postauricular crease [11].

An extended endaural incision has been described as an alternative incision [12]. This incision aimed at making the skin incision away from the tension that may be caused by the body of the implant and the RS; however, skin breakdown at the 
external auditory canal (EAC) and wound infection have been reported as complications of this incision $[11,13,14]$.

The surgical technique of endaural incision in CI should differ from the standard technique used for other otologic surgeries; the standard endaural incision entails incising the skin and periosteum in the same incision line at the incisura and the bony cartilaginous junction of the EAC. Endaural incision for cochlear implantation should be modified. The skin only is incised at the incisura and at the intercartilaginous gap between the conchal cartilage and EAC cartilages (Figure 4), then the skin and the SC tissues are dissected from the underlying pericranium, and then the pericranium is incised away from the site of skin incision. We think that through this modification, endaural incision can be used in CI with lower risk of wound infection or skin breakdown.

\subsection{Periosteal flap elevation}

Few modifications of the standard anterior-based periosteal flap "Palva flap" in CI were described; the aim of these modifications is to ensure both good exposure of the drilling areas (mastoid bone and RS well) and tight periosteal covering of the device at the same time. One of these modifications was described by Fouad et al. [15] in which the periosteum is elevated through two flaps: the first flap is a short anteriorly based periosteal flap that aims at exposure of the mastoid bone, and the second flap is an inferiorly based flap that aims at exposure of the RS bony well (Figure 5). Through this modification, the periosteum can cover the device completely without tension, and mastoid emissary vein disruption could be avoided [15].

\subsection{Mastoidectomy posterior tympanotomy approach}

For more than half a century, the MPTA remains the gold standard approach for CI [16]. However, there is still need for "alternative" approach in certain situations. Also robotic $\mathrm{CI}$ is a new invention that can be used to reduce the need for excess bone drilling and to gain more rapid, safe, and direct access to the RW membrane.

\subsubsection{Other "alternative" approaches}

MPTA is the classic standard approach for CI [17]. Many alternative approaches were described for CI. The most common are the suprameatal approach [18], the pericanal approach [19], transcanal (Veria) approach [20], and transattic approach [21].

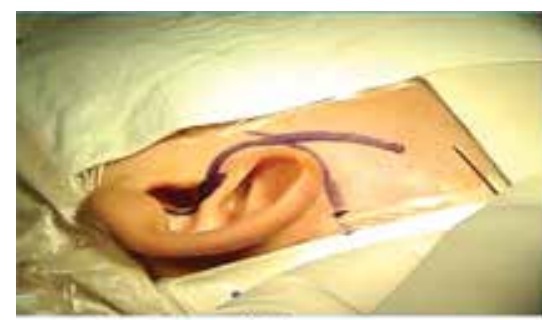

(a)

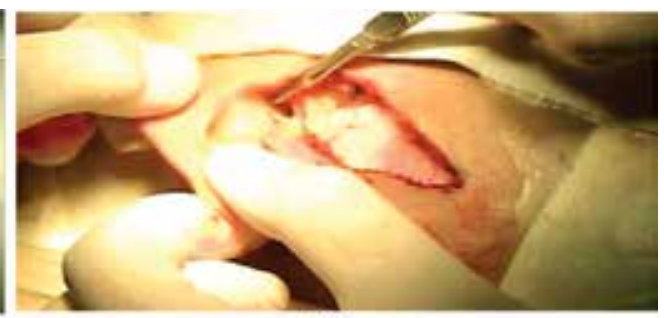

(b)

Figure 4.

Modified endaural incision for CI. (a) Incision marking on the skin (note the transverse part of the incision is at the junction between the conchal cartilage and EAC cartilage) and (b) cutting the transverse part of the incision with scalpel. 


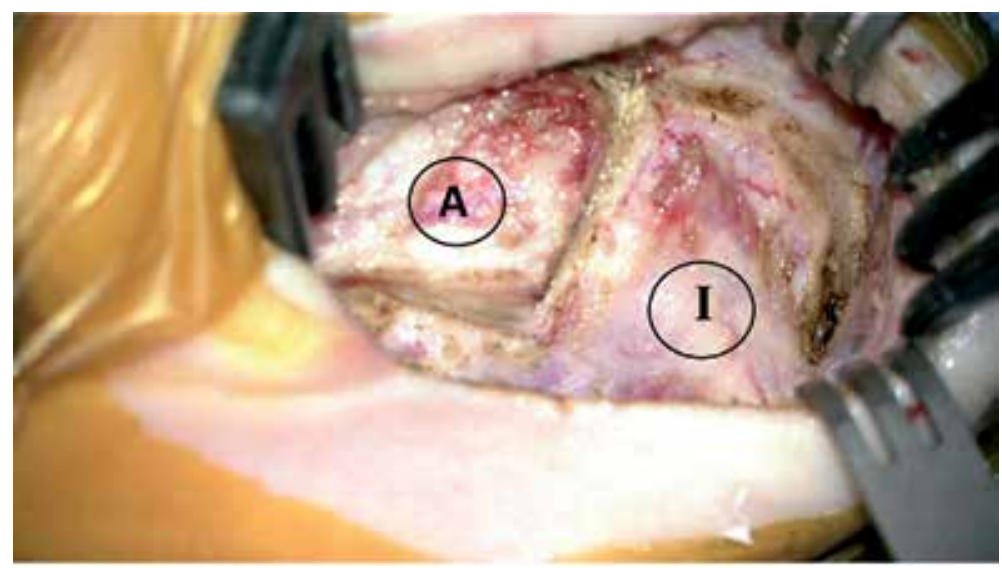

Figure 5.

Modified periosteal flap for CI. Two flaps are taken: (A) anteriorly based flap and (I) inferiorly based flap.

These alternative approaches aim at avoiding the risk of facial nerve injury and decreasing the duration of the surgical procedure. According to El-Anwar et al., there is no significant difference between the reported overall complications rate using either the classic or alternative approaches for CI [22]. However, many authors discourage the non-mastoidectomy approaches for cochlear implantation for the following reasons: First, the angle between the electrode array and the trajectory line of the cochlea is more than $30^{\circ}$; this makes electrode insertion difficult with increasing possibility of intracochlear kinking of the electrode or intracochlear trauma [23]. Second, fixation of the electrode array into a tunnel or groove in the EAC is not suitable for children due to continuous EAC growth [19]. Third, alternative approaches have higher rate of revision cases on the long-term follow-up [11].

Most of the surgeons nowadays use the standard MPTA for CI; the nontraditional approaches for $\mathrm{CI}$ are used in extremely rare cases with difficult anatomical situations [22].

\subsubsection{Robotic surgery in cochlear implantation}

The beginning of the idea of "robotic cochlear implantation" was by thinking in using the navigation system in cochlear implantation through a computerassisted CI surgery using the same classic posterior tympanotomy approach. This idea was first introduced and tried first on cadaver dissection in 2004 [24]; then in 2009, Majdani et al. [25] performed a cadaveric study of using a combination of industrial robot system and navigation system for building a "closed-loop feedback" control system for CI. Through this system they could make real-time feedback to track any movement or changes in the bone based on a preoperative temporal bone CT scan. Vital structures, such as the facial nerve, were defined and protected. The robot was able to drill only the targeted bone without violation of any critical structures [25].

After extensive work and experimental trials for inventing accurate combined robotic and image-guided system for CI [26-28], the minimally invasive robotic percutaneous cochlear implantation (PCI) became real [29].

PCI can modify the classic MPTA into drilling a predesigned small single straight bony tunnel starting from the mastoid cortex and targeting into the RW without risk of injury of the facial nerve, chorda tympani, external auditory canal, and tympanic membrane annulus [30]. 


\subsubsection{Technique of robotic PCI}

According to the first reported case of robotic PCI [29], the procedure starts by preoperative imaging and accurate planning of the drilling pathway and identification of vital structures, before surgery. Then intraoperatively the drill path was assessed using imaging- and sensor-based data to confirm the proximity of the facial nerve. After making the bony tunnel with the robot, a small postauricular incision is made to elevate tympanomeatal flap to expose the RW membrane. The RW membrane is opened through anterior tympanotomy after elevation of the tympanomeatal flap, and then the electrode array, passing through the drilled tunnel, was inserted manually under microscope visualization [29].

\subsubsection{Advantage of robotic PCI}

1. It is a minimal invasive surgery with small wound, short duration, and minimal bone drilling which can cause noise and thermal effect on the cochlea.

2. It has high accuracy rates; the geometric accuracy was measured, in experimental studies, equal to $0.15 \pm 0.08 \mathrm{~mm}$ at the depth of the cochlea.

3. It preserves the mastoid air cells, which has physiological role in middle ear ventilation.

\subsubsection{Disadvantage of robotic PCI}

1. Expensive.

2. Its safety and accuracy in vivo are still under clinical trials.

\subsection{Drilling a bony well for the RS}

The trends in manufacturing recent $\mathrm{CI}$ devices is toward making the RS as thin as possible, so that recent devices are thinner and need drilling a shallower bony well for RS. For example, the thickness of the RS of the CI532® (Cochlear Corp) is $3.9 \mathrm{~mm}$, while the thickness of the RS of the older generation of the same company such as CI124RE® is $6.9 \mathrm{~mm}$. Drilling a bony well with depth equal to $3 \mathrm{~mm}$ is usually enough to accommodate most of recent implants.

Some surgeons advocate the tight "temporalis pocket" technique in fixating the body of the implant; this technique entails elevation of small tight periosteal pocket that can just lodge the device tightly without the need of drilling a well for the RS [31]. Although slim devices can be fixed easier with tight temporalis pocket technique, still most of surgeons prefer drilling a well for stabilization of the RS [16].

Other methods for RS fixation:

- Tie-down sutures that were passed through monocortically drilled holes on each side of the R/S [32].

- Using polypropylene mesh over the R/S and securing the mesh with titanium screws [33].

- Cementing the R/S with ionomeric bone cement [34]. 
- Some CI devices, such as the Neuro Zti ${ }^{\circledR}$ (Oticon Corp), are manufactured with two titanium screws that can be fixed during surgery without the need for drilling a bony well.

\subsection{Cochleostomy}

Insertion of the electrode into the scala tympani is the goal of standard cochlear implantation. To achieve this goal, there are three possible approaches to the scala tympani, each one having advantage and disadvantage:

1. Traditional cochleostomy technique: in which there is a separate opening just inferior and slightly anterior to the RW membrane; it should be crated after good visualization of the RW membrane and lowering down the RW niche. The main advantages of this approach are avoiding the hook region of the basal turn, providing more effective sealing of both cochleostomy and RW by fibrous tissue, and providing appropriate angle of electrode insertion away from the osseous spiral lamina $[5,16]$, However, this approach entails more bone drilling on the cochlea that may expose the neuro-sensitive structures of the cochlea to traumatic and thermal effect of the drill [35].

2. RW approach: in which the RW membrane is opened, better by using a sharp needle. This approach is the least traumatic approach; however, electrode insertion may be difficult, and electrode may be hanged in the hook region by a projecting crista fenestra, which will need further drilling to allow electrode insertion.

3. Extended RW approach: in which the round window membrane is opened and then the anterior-inferior margin of the RW is drilled till good visualization of the scala tympani is achieved. Through this approach, the hook region is avoided, electrode array insertion will be in the same trajectory line of the scala tympani, and trauma to the osseous spiral lamina will be avoided.

The "best" type of cochleostomy is still a controversial issue; however, according to the meta-analysis conducted by Santa Maria et al., hearing preservation rates were higher in cochleostomy than in RW approach [36]. Whatever the surgical approach used for cochleostomy, the key point for successful scala tympani insertion with minimal trauma is good access and visualization of the whole round window membrane.

However, the RW visibility through the surgical microscope through MPTA is variable. St Thomas' Hospital introduced a classification for the visibility of the RW during CI as follows: type I, the RW membrane is entirely exposed; type IIa, more than $50 \%$ but less than $100 \%$ of the RW membrane is exposed; type IIb, the exposure of RW membrane is less than $50 \%$ but more than $0 \%$; and type III, the RW membrane could not be identified. Most of the adult cases (76\%) were type I, $17 \%$ was type IIa and IIb, while 7\% was type III [37].

\subsubsection{Endoscopic cochlear implantation}

Otoendoscopy can be used, instead of surgical microscope, to solve the problem of "difficult RW." Marchioni et al. [38] has described the surgical technique of endoscopic CI. They used $3 \mathrm{~mm}$ rigid otoscope through the EAC, after elevation of an intact tympanomeatal flap, without incising the EAC skin, and then endoscopic cochleostomy is performed through the RW membrane. However, they did not use 
MPTA for electrode insertion; instead of that, they used pericanal approach by drilling a bony grove in the posterior wall of EAC [38].

However, due to the advantages of the standard MPTA, the use of endoscope in CI became mainly limited to help the surgeons in accurate identification of the RW membrane and precise electrode placement; also this technique appears to be particularly useful for malformed or abnormal cochlea [39-41].

\subsection{Electrode insertion}

\subsubsection{Types of CI electrode arrays}

According to the method of insertion of the CI electrode array, there are three main types of CI electrode arrays that vary in the design and the method of the insertion:

1. The lateral wall (LW) electrode: Such as the K electrode of the Nucleus ${ }^{\circledR}$ (Cochlear Corp, Lane Cove, Australia), all MED-EL electrodes (MED-EL Corp, Innsbruck, Austria), and the HiFocus ${ }^{\mathrm{TM}} 1 \mathrm{~J}$ Electrode (Advanced Bionics Corp, Sylmar, CA). The lateral wall electrode, with exception of the $1 \mathrm{~J}$ electrode, is usually inserted by using micro forceps with or without the guide of claw instrument. The $1 \mathrm{~J}$ Electrode is better to be inserted with its specific pre-loaded metal tube connecting to its specific applicator.

2. The midscalar (MS) electrode: such as Mid-Scala Electrode of HiFocus ${ }^{\mathrm{TM}}$ (Advanced Bionics Corp,). This type of electrode is usually inserted through a specific applicator using the off-stylet technique (that was originally described for the Contour Advance electrode ${ }^{\circledR}$ (Cochlear Corp) [42].

3. The perimodiolar (PM) electrode: such as HiFocus Helix ${ }^{\mathrm{TM}}$ electrode (Advanced Bionics Corp) and the Contour ${ }^{\circledR}$ electrode (Cochlear Corp). Both of these electrodes have a stylet that is removed during insertion by the off-stylet technique. The recent CI532® (Cochlear Corp) is a PM slim electrode $(0.7 \mathrm{~mm})$; during insertion the electrode is loaded in its sheath, the stopper is kept at the cochleostomy opening, and then the electrode array is slowly advanced out of the electrode sheath. The electrode sheath was then removed, after seeing the three white markers at the cochleostomy site [43].

Each of the three types of electrode array has advantages and disadvantages. In general, the LW electrodes are usually slimmer and are assumed to have less traumatic effect on the cochlea during insertion, but they are usually rest away from the spiral ganglia which are the target of the electrodes' stimulatory impulses. The PM electrodes can hug the modiolus and became very close to the spiral ganglia; but because of the need of stylet, they are usually more stiff and thick, except the new PM electrode generations such as CI532 ${ }^{\circledR}$ (Cochlear Corp), so that PM electrodes usually have more traumatic potentials on the cochlea during insertion. The MS electrodes are assumed to have the advantages of both LW and PM electrodes, but they can also have the disadvantages of both of them [44-46].

\subsubsection{Depth of insertion of the CI electrode array}

Proper electrode insertion is achieved by both making full insertion, which entails inserting all active electrodes into the scala tympani, and by making appropriate depth of insertion. Regarding the depth of insertion, Yukawa et al. 
[47] reported that the better predictor of the outcome for the depth of electrode insertion is the angular depth rather than the intracochlear length of the electrode or even the number of active electrodes that is used in speech processing. It is expected that LW electrodes, especially the long types as FlexSoft ${ }^{\mathrm{TM}}$ (MED-EL Corp), can demonstrate greater angular insertion depth, more than $360^{\circ}$, while the $\mathrm{PM}$ or MS electrodes are usually designed to encircle the first basal turn making angular insertion depth nearly equal to $360^{\circ}$ [48]. Insertion depth at $360^{\circ}$, or less than one cochlear turn, is usually associated with poor speech outcome; however, above $360^{\circ}$, there is no association between the depth of insertion and the speech outcome $[49,50]$. Deep insertion is assumed to have the advantage of extending the electrical stimulation into the apical region that is responsible for low-frequency sounds; this provides better place pitch match which may improve the outcomes of CI especially in the music perception [51]. However, deep insertion is usually associated with increasing the risk of intracochlear trauma [45].

In conclusion, the best design for "ideal" CI electrode is a matter of debate; there is no "best" CI electrode for all CI cases.

\subsection{Fixation of the implant and then testing the device function}

Fixation of the implant entails both fixation of the RS, the main body of the implant, and fixation of the electrode array. Fixation of the RS was mentioned before, but whatever is the technique used, the periosteum should be tightened and sewed over the implant during this step [7].

Fixation of the electrode array should be in both its proximal and distal ends; the proximal end is fixed simply by either drilling a deep groove or trough starting from the RS bony well at the site of exit of the electrode to the mastoid cavity [52]. This trough could be drilled deeper. As it reaches the mastoid cavity at the sino-dural angle, the bone at this area is thick, so the trough can be modified in this area into incomplete tunnel with a bony ledge. The electrode could be secured in this tunnel even after electrode insertion (Figure 6).

The distal end of electrode array is fixed routinely by both inserting fascial plug around the electrode at the cochleostomy site and also by placing a loop of electrode

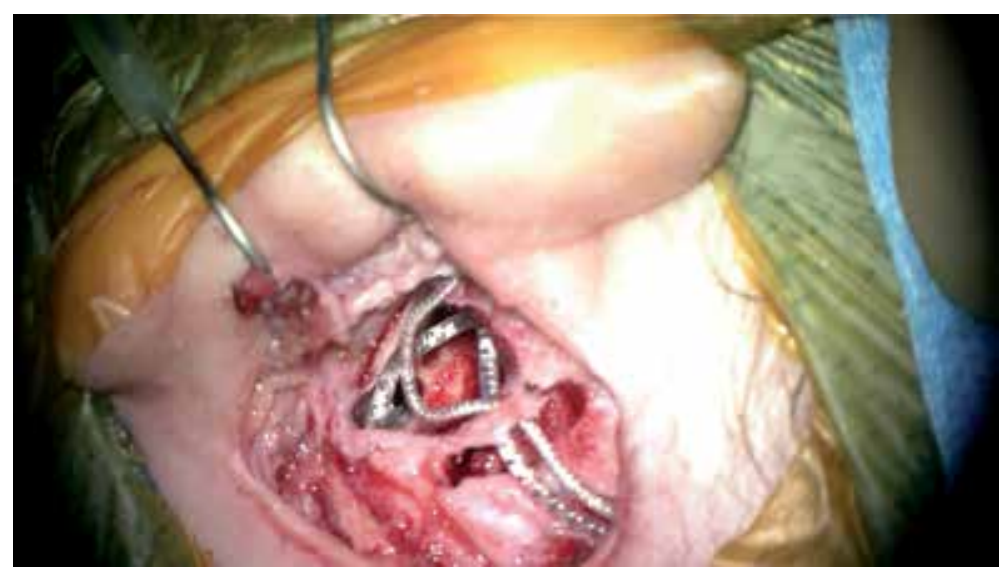

Figure 6.

The electrode is secured at the sino-dural angle before entering the mastoidectomy cavity, by an incomplete tunnel with a bony ledge. 


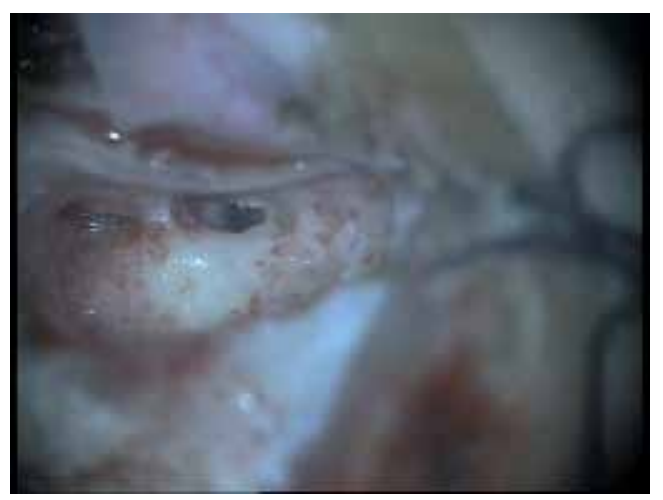

Figure 7 .

A groove in the lower end of the posterior tympanotomy for accommodation and stabilization of the electrode after insertion.

cable against the tegmen mastoideum. Other surgical techniques that can be used, in addition, for electrode fixation at its distal end are:

1. Using a titanium clip to attach the electrode array to the incus bar [53].

2. The "split-bridge" technique [54], in which a channel is made through the incus bar and the lead wedged in it.

3. Making a small inferiorly based bone grove in the posterior tympanotomy into which the electrode array can be squeezed for fixation [55]. The groove is made with $1 \mathrm{~mm}$ diamond burr between the facial nerve and the chorda tympani nerve (Figure 7).

\subsection{Intraoperative device monitoring}

The use of the Internet for monitoring of CI devices from remote locations is a recent advance in CI programming. In a study by Shapiro et al. [56] remote intraoperative CI device monitoring was compared to in situ monitoring. The results showed that there is a significant reduction of the audiologist's time with remote testing than in situ testing. This represents a significant reduction in time required for testing and consequently the cost. This can be achieved by only Internet connection and a telephone [57].

\section{Advances of the anesthetic techniques}

\subsection{CI under conscious sedation with local anesthesia (CS-LA)}

Toner et al. [58] reported a case series of cochlear implantation under local anesthesia; however, using local anesthesia in CI was not widely used till the last 10 years [59-63], especially after the introduction and widespread usage of dexmedetomidine as a sedative drug for cases of CS-LA. Dexmedetomidine can make "cooperative sedation," in which the patient remains arousable and cooperative without causing delirium and unnecessary movements associated with delirium [63]. 
CI under CS-LA achieved comparable results with general anesthesia (GA) regarding perioperative comorbidities and achieved better results than GA regarding patient satisfaction in elderly patient [63].

\subsection{Indications and advantages of CS-LA in CI}

CS-LA is not only indicated in patients who are unfit for GA, but also it is generally preferred than GA in elderly patients. CI under CS-LA has the following advantages in elderly patients [63]:

1. CS-LA was associated with decreased drug costs, surgery time, and anesthesia time.

2. Length of stay was significantly shorter in patients undergoing CI under CS-LA.

3. Patient satisfaction was superior with CS-LA.

4. Perioperative morbidity was higher, but not significant, with GA than CS-LA.

5. GA in elderly patient carries the risk of unexpected cognitive consequences after surgery.

\subsection{Technique}

According to Shabashev et al. [63], the patient receives dexmedetomidine as the main sedative drug, in addition to fentanyl, midazolam, lidocaine, and propofol, depending on the necessary level of sedation and analgesia. In addition, 8-10 mL of 2\% lidocaine with 1:100,000 epinephrine was used as a local infiltration anesthesia. In some instances, when patients experienced additional pain upon exposing the middle ear mucosa, gelfoam pledges soaked in the same local anesthetic solution were applied directly to the middle ear mucosa for 2 minutes before continuing the manipulation. Supplemental oxygen less than $29 \%$ was administered via nasal cannula or face mask. Surgical drape was applied around the operative site, but the face was left completely uncovered to facilitate direct communication with the patient during the procedure [63].

\section{Hearing preservation during cochlear implantation}

\subsection{Surgical technique}

All the previously described refinements and advances in both surgical techniques and electrode design aim at increasing the performance of the electrode within the cochlea and decreasing the traumatic effect of the electrode on the residual neuro-sensitive structures in the cochlea; this can preserve the residual function of these structures aiming at hearing preservation. Through these surgical refinements, nontraumatic "soft" CI surgery can achieve the target of hearing preservation.

In addition to the previously described technical refinements, there are many surgical considerations that should be respected during performing nontraumatic "soft" CI surgery. The most important surgical considerations are: 
1. Minimal bone drilling during cochleostomy and avoidance of entrance of bone dusts into cochlear lumen [35].

2. Careful dealing with the endosteum during cochleostomy by incising it sharply using a sharp needle [64].

3. Scala tympani electrode insertion and avoidance of injury of the osseous spiral lamina and basilar membrane [65].

4. Preservation of the perilymph in scala tympani by avoiding suction of the perilymph during cochleostomy [16].

5. Smooth and slow electrode insertion [36].

6. In case of using long electrode, avoid excess pressure on electrode during insertion that may cause intracochlear trauma [45].

7. Electrode fixation and stabilization [7].

8. Sealing of the cochleostomy with soft tissue seal to avoid perilymph leakage [36].

\subsection{Pharmacotherapy for hearing preservation during CI}

Corticosteroid can be administrated during CI surgery aiming at hearing preservation [65]. Perioperative corticosteroids can be used either systemic, intratympanic, or intracochlear. Systemic steroid can be used either intravenously during the surgery or orally after the surgery [64]. Postoperative oral corticosteroid improved hearing preservation rates according to the systematic review conducted by Santa et al. [36].

Intratympanic steroid has been described through either applying a gelfoam soaked with methylprednisolone $125 \mathrm{mg} / \mathrm{ml}$ over RW membrane for 30 minutes before cochleostomy [66] or by filling the middle ear by dexamethasone $4 \mathrm{mg} / \mathrm{ml}$ before electrode insertion [67].

Intracochlear corticosteroid has been described through either using intracochlear injection of triamcinolone acetonide solution in addition to hyaluronic acid [68] or through silicone-based dexamethasone-eluted cochlear implant [69].

Experimental animal study on corticosteroid-eluted cochlear implant devices showed significant hearing preservation rates and histopathologic evidence of lower inflammatory response to the electrode [70-72]. However, until nowadays, many authors still discourage the use of intracochlear or intratympanic corticosteroids during CI surgery [16].

In addition to corticosteroid, other drugs can be administrated through drug-eluted CI device. There are many ongoing experimental trials on intracochlear application of neurotrophins and antiapoptotic drugs through drug-eluted CI device [72].

\subsection{Effect of hearing preservation surgery on CI outcomes}

CI surgery was introduced first as the only solution for hearing loss in profound deaf subjects. The US Food and Drug Administration first approved CI for adults with postlingual profound bilateral sensorineural hearing loss in 1985 and children in 1990. Nowadays, with the refinement of the surgical techniques and the advances of electrode design, CI candidacy guidelines have been expanded to include adults and children with residual hearing in the implanted ear [73]. 
This expansion in CI candidacy criteria was based on the strong evidence of two hypotheses: the first is the ability of CI surgery to preserve the residual hearing, and the second is the beneficial effect and the better speech outcomes of CI surgery in ears with residual hearing.

Systematic review studies were conducted on the effect of CI surgical techniques on hearing preservations $[36,65,74]$; all of these studies gathered that nontraumatic "soft" CI surgery can preserve hearing. Gantz et al. conducted a multicenter study on the outcome of CI surgery on 87 ears with residual hearing. At initial activation, $90 \%$ of the subjects maintained a functional low-frequency pure-tone average; this percentage was reduced to $80 \%$ after 12 months [75].

Regarding the benefit of CI in ears with residual hearing, a systematic review study was conducted on the outcome of $\mathrm{CI}$ in children with residual hearing; this study demonstrated that the better the preoperative residual hearing, the better the postoperative speech perception outcomes [76].

\section{Conclusion}

More than half a century passed since the first cochlear implantation surgery; throughout this long period, the main advances happened in cochlear implantation were the manufacture of the implant itself, surgical technique showing a lot of refinement rather than changes, and also the possibility of surgery nowadays to be performed under local anesthesia. The ongoing advances in cochlear implants and refinements of the surgical techniques have improved the outcomes of cochlear implantation and allowed for hearing preservation in case of preoperative residual hearing.

\section{Acknowledgements}

Roland Jr., J.T. had major role in this work; he helped me as a mentor, and also he provided me with four figures of his own work (Figures 1, 2, 5, and 6).

\section{Conflict of interest}

The authors declare no conflict of interest.

\section{Abbreviations}

CI

MPTA

RW

CS-LA

GA

RS

ESRT

ECAP

NRT

PCI

EAC

LW

MS

PM cochlear implantation

mastoidectomy posterior tympanotomy approach

round window

conscious sedation with local anesthesia

general anesthesia

receiver/stimulator

electrical stapedial reflex thresholds

electrical compound action potential

neural response telemetry

percutaneous cochlear implantation

external auditory canal

lateral wall

midscalar

perimodiolar 


\section{Author details}

Yasser A. Fouad

Faculty of Medicine, Zagazig University, Zagazig, Egypt

*Address all correspondence to: yasserfoadent@gmail.com

\section{IntechOpen}

(C) 2020 The Author(s). Licensee IntechOpen. This chapter is distributed under the terms of the Creative Commons Attribution License (http://creativecommons.org/licenses/ by/3.0), which permits unrestricted use, distribution, and reproduction in any medium, provided the original work is properly cited. (cc) BY 


\section{References}

[1] Clark GM. Introduction. In: Cochlear Implants: Fundamentals and Applications. New York: SpringerVerlag; 2003. pp. 32-39

[2] Blume SS. Histories of cochlear implantation. Social Science and Medicine. 1999;49(9):1257-1268

[3] House WF. Cochlear implants. The Annals of Otology, Rhinology, and Laryngology. 1976;85(3_suppl):3-3

[4] Flint P, editor. Cummings Otolaryngology_-Head and Neck Surgery. 5th ed. Philadelphia: Mosby, Elsevier; 2010. pp. 2237-2239

[5] Clark GM. Surgery. In: Cochlear Implants: Fundamentals and Applications. New York: SpringerVerlag; 2003. pp. 595-653

[6] Roland P, Sabatini PR. Cochlear Implant Surgery: The Traditional Approach and its Alternatives. Implantable Hearing Devices; 2017. p. 51

[7] Molony TB, Giles JE, Thompson TL, Motamedi KK. Device fixation in cochlear implantation: Is bone anchoring necessary? The Laryngoscope. 2010;120(9):1837-1839

[8] Connell SS, Balkany TJ, Hodges AV, et al. Electrode migration after cochlear implantation. Otology and Neurotology. 2008;29:156-159

[9] Shapiro WH, Bradham TS. Cochlear implant programming. Otolaryngologic Clinics of North America. 2012;45(1): 111-127

[10] Cosetti MK, Shapiro WH, Green JE, Roman BR, Lalwani AK, Gunn SH, et al. Intraoperative neural response telemetry as a predictor of performance. Otology and Neurotology. 2010;31(7):1095-1099
[11] Mangus B, Rivas A, Tsai BS, Haynes DS, Roland JT. Surgical techniques in cochlear implants. Otolaryngologic Clinics of North America. 2012;45(1):69-80

[12] Franz BKH, Lehnhardt E, Patrick JF, Kuzma JA, Clark GM, Laszig R. Implantation of the Melbourne/cochlear multiple-electrode extracochlear prosthesis. The Annals of Otology, Rhinology, and Laryngology. 1989;98(8):591-596

[13] Sorrentino T, Côté M, Eter E, Laborde ML, Cochard N, Deguine O, etal. Cochlear reimplantations: Technical and surgical failures. Acta OtoLaryngologica. 2009;129(4):380-384

[14] Gibson WPR, Harrison HC. Further experience with a straight, vertical incision for placement of cochlear implants. The Journal of Laryngology and Otology. 1997;111(10):924-927

[15] Fouad YA, Roland JT Jr. Periosteal flap in cochlear implantation, how I do it? The Journal of International Advanced Otology. 2018;14(1):140

[16] Roland PS, Roland JT Jr. 10 Cochlear Implant Surgical. Cochlear Implants. 2014

[17] Zeitler DM, Balkany TJ. Alternative approaches to cochlear implantation.

Operative Techniques in

Otolaryngology-Head and Neck Surgery. 2010;21(4):248-253

[18] Kronenberg J, Migirov L, Dagan T. Suprameatal approach: New surgical approach for cochlear implantation. The Journal of Laryngology and Otology. 2001;115(4):283-285

[19] Häusler R. Cochlear implantation without mastoidectomy: The 
pericanal electrode insertion technique. Acta Oto-Laryngologica. 2002;122(7):715-719

[20] Kiratzidis T, Arnold W, Iliades T. Veria operation updated. ORL. 2002;64(6):406-412

[21] Vaca M, Gutiérrez A, Polo R, Alonso A, Álvarez F. Long-term results of the transattical approach: An alternative technique for cochlear implantation. European Archives of Oto-Rhino-Laryngology. 2015;272(1):35-41

[22] El-Anwar MW, ElAassar AS, Foad YA. Non-mastoidectomy cochlear implant approaches: A literature review. International Archives of Otorhinolaryngology. 2016;20(02):180-184

[23] Postelmans JT, Grolman W, Tange RA, Stokroos RJ. Comparison of two approaches to the surgical management of cochlear implantation. The Laryngoscope. 2009;119(8):1571-1578

[24] Schipper J, Aschendorff A, Arapakis I, Klenzner T, Teszler CB, Ridder GJ, et al. Navigation as a quality management tool in cochlear implant surgery. The Journal of Laryngology and Otology. 2004;118(10):764-770

[25] Majdani O, Rau TS, Baron S, Eilers H, Baier C, Heimann B, et al. A robot-guided minimally invasive approach for cochlear implant surgery: Preliminary results of a temporal bone study. International Journal of Computer Assisted Radiology and Surgery. 2009;4(5):475-486

[26] Labadie RF, Balachandran R, Mitchell JE, et al. Clinical validation study of percutaneous cochlear access using patient-customized microstereotactic frames. Otology and Neurotology. 2010;31(1):94-99
[27] Balachandran R, Mitchell JE, Blachon GS, Noble JH, Dawant BM, Fitzpatrick JM, et al. Percutaneous cochlear implant drilling via customized frames: An in vitro study. Otolaryngology and Head and Neck Surgery. 2010;142:421-426

[28] Balachandran R, Mitchell JE, Nobel $\mathrm{J}$ et al. Insertion of electrode array using percutaneous cochlear implantation technique: A cadaveric study. In: Proc SPIE; Vol. 7964. 2011. p. 79641

[29] Caversaccio M, Gavaghan K, Wimmer W, Williamson T, Anso J, Mantokoudis G, et al. Robotic cochlear implantation: Surgical procedure and first clinical experience. Acta OtoLaryngologica. 2017;137(4):447-454

[30] McRackan TR, Labadie RF, Thomas J. 11 New Horizons in Surgical. Cochlear Implants. 2014

[31] Balkany TJ, Whitley M, Shapira Y, et al. The temporalis pocket technique for cochlear implantation: An anatomic and clinical study. Otology and Neurotology. 2009;30:903-907

[32] Cohen NL, Roland JT Jr, Fishman A. Surgical technique for the nucleus contour cochlear implant. Ear and Hearing. 2002;23:59S-66S

[33] Davis BM, Labadie RF, McMenomey SO, et al. Cochlear implant fixation using polypropylene mesh and titanium screws. Laryngoscope. 2004;114:2116-2118

[34] Rudel C, Zollner W. Ionomeric cement-A bone glue for device fixation. Ear, Nose, and Throat Journal. 1994;73:189-191

[35] Pau HW, Just T, Bornitz M, Lasurashvilli N, Zahnert T. Noise exposure of the inner ear during drilling a cochleostomy for cochlear implantation. The Laryngoscope. 2007;117(3):535-540 
[36] Santa Maria PL, Gluth MB, Yuan Y, Atlas MD, Blevins NH. Hearing preservation surgery for cochlear implantation: A meta-analysis. Otology and Neurotology. 2014;35(10):e256-e269

[37] Leong AC, Jiang D, Agger A, Fitzgerald-O'Connor A. Evaluation of round window accessibility to cochlear implant insertion. European Archives of Oto-Rhino-Laryngology. 2013;270(4):1237-1242

[38] Marchioni D, Grammatica A, Alicandri-Ciufelli M, Genovese E, Presutti L. Endoscopic cochlear implant procedure. European Archives of Oto-Rhino-Laryngology. 2014;271(5):959-966

[39] Rajan P, Teh HM, Prepageran N, Kamalden TIT, Tang P. Endoscopic cochlear implant: Literature review and current status. Current Otorhinolaryngology Reports. 2017;5(4):268-274

[40] Marchioni D, Soloperto D, Bianconi L, Guarnaccia MC, Genovese E, Presutti L. Endoscopic approach for cochlear implantation in advanced otosclerosis: A case report. Auris Nasus Larynx. 2016;43(5):584-590

[41] Marchioni D, Carner M, Soloperto D, Sacchetto A, Genovese E, Presutti L. Endoscopic-assisted cochlear implant procedure in CHARGE syndrome: Preliminary report. Acta Oto-Laryngologica Case Reports. 2017;2(1):52-58

[42] Roland JT, Shevla M, Gibson P, Treaba C. Electrode insertion mechanics and outer wall forces with the nucleus 24 contour advanceTM electrode. Cochlear Implants International. 2005;6(sup1):5-8

[43] Friedmann DR, Kamen E, Choudhury B, Roland JT Jr. Surgical experience and early outcomes with a slim perimodiolar electrode. Otology and Neurotology. 2019;40(3):e304-e310

[44] Roland JT Jr. Cochlear implant electrode insertion. Operative Techniques in Otolaryngology-Head and Neck Surgery. 2005;16(2):86-92

[45] Adunka O, Kiefer J. Impact of electrode insertion depth on intracochlear trauma. Otolaryngology and Head and Neck Surgery. 2006;135(3):374-382

[46] Cuda D, Murri A. Cochlear implantation with the nucleus slim modiolar electrode (CI532): A preliminary experience. European Archives of Oto-Rhino-Laryngology. 2017;274(12):4141-4148

[47] Yukawa K, Cohen L, Blamey P, Pyman B, Tungvachirakul V, O'Leary S. Effects of insertion depth of cochlear implant electrodes upon speech perception. Audiology and Neurotology. 2004;9(3):163-172

[48] O'Connell BP, Hunter JB, Haynes DS, Holder JT, Dedmon MM, Noble JH, et al. Insertion depth impacts speech perception and hearing preservation for lateral wall electrodes. The Laryngoscope. 2017;127(10):2352-2357

[49] Hilly O, Smith L, Hwang E, Shipp D, Symons S, Nedzelski JM, et al. Depth of cochlear implant array within the cochlea and performance outcome. The Annals of Otology, Rhinology, and Laryngology. 2016;125(11):886-892

[50] Van Der Marel KS, Briaire JJ, Verbist BM, Muurling TJ, Frijns JH. The influence of cochlear implant electrode position on performance. Audiology and Neurotology. 2015;20(3):202-211

[51] Hochmair I, Arnold W, Nopp P, Jolly C, Müller J, Roland P. Deep electrode insertion in cochlear implants: Apical morphology, electrodes and 
speech perception results. Acta OtoLaryngologica. 2003;123(5):612-617

[52] Ramsden RT. Cochlear implant surgery. In: Cooper HR, Craddock LC, editors. Cochlear Implants: A Practical Guide. John Wiley \& Sons; 2006. pp. 216-242

[53] Cohen NL. Titanium clip for cochlear implant electrode fixation. The Annals of Otology, Rhinology, and Laryngology. Sep 1995;166:402-403

[54] Balkany T, Telischi FF. How I do it: Otology and neurotology: Fixation of the electrode cable during cochlear implantation: The split bridge technique. The Laryngoscope. 1995;105(2):217-218

[55] Lenarz T, Stöver T, Buechner A, Lesinski-Schiedat A, Patrick J, Pesch J. Hearing conservation surgery using the hybrid-L electrode. Audiology and Neurotology. 2009;14(Suppl. 1):22-31

[56] Shapiro WH, Huang T, Shaw T, Roland JT, Lalwani AK. Remote intraoperative monitoring during cochlear implant surgery is feasible and efficient. Otology and Neurotology. 2008;29:49S-498S

[57] Shapiro WH. Advancements in cochlear implant programming. Cochlear Implants. 2014. pp. 148-157

[58] Toner JG, John G, McNaboe EJ. Cochlear implantation under local anaesthesia, the Belfast experience. The Journal of Laryngology and Otology. 1998;112(6):533-536

[59] Dietz A, Wüstefeld M, Niskanen M, Löppönen H. Cochlear implant surgery in the elderly: The feasibility of a modified suprameatal approach under local anesthesia. Otology and Neurotology. 2016;37(5):487-491

[60] Hamerschmidt R, Moreira ATR, Wiemes GRM, Tenório SB,
Tâmbara EM. Cochlear implant surgery with local anesthesia and sedation: Comparison with general anesthesia. Otology and Neurotology. 2013;34(1):75-78

[61] Pateron B, Bakhos D, LeLouarn A, Bordure P, Grayeli AB, Godey B, et al. Local anaesthesia and conscious sedation for cochlear implantation: Experience with 20 patients. The Journal of Laryngology and Otology. 2015;130(2):151-156

[62] Svrakic M, Pollack A, Huncke TK, Roland JT. Conscious sedation and local anesthesia for patients undergoing neurotologic and complex otologic procedures. Otology and Neurotology. 2014;35(10):e277-e285

[63] Shabashev S, Fouad Y, Huncke TK, Roland JT. Cochlear implantation under conscious sedation with local anesthesia; safety, efficacy, costs, and satisfaction. Cochlear Implants International. 2017;18(6):297-303

[64] Roland PS, Gstöttner W, Adunka O. Method for hearing preservation in cochlear implant surgery. Operative Techniques in Otolaryngology-Head and Neck Surgery. 2005;16(2):93-100

[65] Khater A, El-Anwar MW. Methods of hearing preservation during cochlear implantation. International Archives of Otorhinolaryngology. 2017;21(03):297-301

[66] Enticott JC, Eastwood HT, Briggs RJ, Dowell RC, O’Leary SJ. Methylprednisolone applied directly to the round window reduces dizziness after cochlear implantation: A randomized clinical trial. Audiology and Neurotology. 2011;16(5):289-303

[67] Cho HS, Lee KY, Choi H, Jang JH, Lee SH. Dexamethasone is one of the factors minimizing the inner ear damage from electrode insertion in cochlear implantation. Audiology and Neurotology. 2016;21(3):178-186 
[68] De Ceulaer G, Johnson S, Yperman M, Daemers K, Offeciers FE, O'Donoghue GM, et al. Long-term evaluation of the effect of intracochlear steroid deposition on electrode impedance in cochlear implant patients. Otology and Neurotology. 2003;24(5):769-774

[69] Liu Y, Jolly C, Braun S, Stark T, Scherer E, Plontke SK, et al. In vitro and in vivo pharmacokinetic study of a dexamethasone-releasing silicone for cochlear implants. European Archives of Oto-Rhino-Laryngology. 2016;273(7):1745-1753

[70] Lyu AR, Kim DH, Lee SH, Shin DS, Shin SA, Park YH. Effects of dexamethasone on intracochlear inflammation and residual hearing after cochleostomy: A comparison of administration routes. PLoS One. 2018;13(3):e0195230

[71] Farhadi M, Jalessi M, Salehian P, Ghavi FF, Emamjomeh H, Mirzadeh H, et al. Dexamethasone eluting cochlear implant: Histological study in animal model. Cochlear Implants International. 2013;14(1):45-50

[72] Plontke SK, Götze G, Rahne T, Liebau A. Intracochlear drug delivery in combination with cochlear implants. HNO. 2017;65(1):19-28

[73] Carlson ML, Patel NS, Tombers NM, DeJong MD, Breneman AI, Neff BA, et al. Hearing preservation in pediatric cochlear implantation. Otology and Neurotology. 2017;38(6):e128-e133

[74] Havenith S, Lammers MJ, Tange RA, Trabalzini F, della Volpe A, van der Heijden GJ, et al. Hearing preservation surgery: Cochleostomy or round window approach? A systematic review. Otology and Neurotology. 2013;34(4):667-674

[75] Gantz BJ, Dunn C, Oleson J, Hansen M, Parkinson A, Turner C.
Multicenter clinical trial of the nucleus hybrid S8 cochlear implant: Final outcomes. The Laryngoscope. 2016;126(4):962-973

[76] Chiossi JSC, Hyppolito MA. Effects of residual hearing on cochlear implant outcomes in children: A systematic-review. International Journal of Pediatric Otorhinolaryngology. 2017;100:119-127 


\title{
Prognostics Factors of Cochlear Implant in Adults: How Can We Improve Poorer Performers?
}

\author{
Bernard Fraysse and Chris J. James
}

\begin{abstract}
Rehabilitation for any cochlear implant (CI) recipient is a process having the aim of taking the necessary steps to enable users to achieve their best possible auditory outcome. It involves all stages of interaction including evaluations for candidacy, device selection, preoperative counseling, surgical intervention, device activation, post-implant support, evaluations of progress, and hearing training. Since rehabilitation is an ongoing process, it becomes critical to determine what is sufficient, that is, how intense the follow-up program must be, especially since there is substantial variability for results on outcome measures that assess progress in hearing function and abilities after implantation.
\end{abstract}

Keywords: rehabilitation, cochlear implant

\section{Introduction}

Rehabilitation for any cochlear implant (CI) recipient is a process having the aim of taking the necessary steps to enable users to achieve their best possible auditory outcome. It involves all stages of interaction including evaluations for candidacy, device selection, preoperative counseling, surgical intervention, device activation, post-implant support, evaluations of progress, and hearing training. Since rehabilitation is an ongoing process, it becomes critical to determine what is sufficient, that is, how intense the follow-up program must be, especially since there is substantial variability for results on outcome measures that assess progress in hearing function and abilities after implantation [4].

A primary aspect of our rehabilitation approach is early identification of the challenges associated with the remediation of adult cochlear implant users who demonstrate poor results on objective measures. A poor performer may be described as one who achieves “... limited performance after taking in account the preoperative biographic factors during counselling the patient and anatomical factors electrode insertion" [1].

The approach we present in this chapter is based on years of experience and research in our facility and in cooperation with other multicenter studies. Nearly $50 \%$ of our patient population will be managed, postoperatively, through conventional (passive) auditory rehabilitation, which means managing all aspects related to device use and counseling and encouraging patient-driven practices. Patientdriven practices take advantage of everyday life encounters, whether it be through 
exposure to TV and videos/movies without captioning, audiobooks, telephone use, social media communication applications (Skype, FaceTime, WhatsApp, etc.), family encounters at group get-togethers, one-on-one with co-workers or friends and family, etc. [2]. The remaining patients will require further, detailed investigations and personalized active rehabilitation. Experience shows that the early identification of those requiring more active rehabilitation training leads to better outcomes. It results in a reduction in the number of visits for those requiring less direct intervention and allows our clinical specialists to concentrate on improving the outcomes of poorer performers.

\section{Preoperative counseling}

Although candidates receive comprehensive counseling throughout their rehabilitation program, the pre-implant sessions lay the foundation for establishing realistic expectations. This goal is supported by employing a predictive model from which the basis for expectations can be established. The model predicts the sentence recognition score of CI users 1 month after activation. It was derived and simplified from the analysis by [1]. The model takes into consideration only the duration of severe-to-profound hearing loss (HL) and one key etiology, congenital hearing loss, which produced significantly lower scores than other etiologies (including "unknown" causes). The formula is easily applied:

$$
\text { Predicted score }=90-0.5 \times \text { years } \mathrm{HL}-50 \text { (if congenital } \mathrm{HL} \text { ) }
$$

where 90 represents the expected score (out of one hundred) for a good performer, which is reduced by 0.5 points per year of severe-to-profound hearing loss and further reduced by 50 points if the etiology was congenital HL. If the etiology is not congenital, then the formula is only 90 minus half the number of years of HL. The predictive model is not valid for cases of labyrinthitis (e.g., chronic otitis and autoimmune disease), where the findings of [1] indicated considerable variability and generally poor outcomes. Our evidence from adults suggests a priori that those with congenital HL are expected to yield poor performance scores. As an example, for deafness acquired in adulthood for a duration of 40 years, the prediction would be a score of 70; however, if the deafness had been congenital, the score would be 20. Another example for a person with short-term deafness of 6 years would yield a higher score $(90-3=87)$. The predictions are valid assuming that the best surgical outcome is obtained in terms of electrode array position and insertion depth (see below).

As will be discussed later, outcome scores could be worse than expected for any CI user and would indicate the need for ongoing rehabilitation intervention. Individuals with poorer than expected scores would be considered poor users. In other words, additional factors may intervene with the duration of deafness and etiology to affect the results, many of which can be investigated and evaluated only after implantation.

Based on the population data from [1], we generated a distribution of scores assuming ideal electrode position before activation, that is, no dislocation and insertion depth within the recommended limits. The resultant median score was at approximately 70/100 (Figure 1, raw scores, left).

If there is to be some effect on the overall population performance, we need to choose a relatively high threshold below which we will apply active rehabilitation. The rationale is that bringing up the performance of the lowest half of the population is a worthy, and likely, achievable aim, and, therefore, we set the threshold 


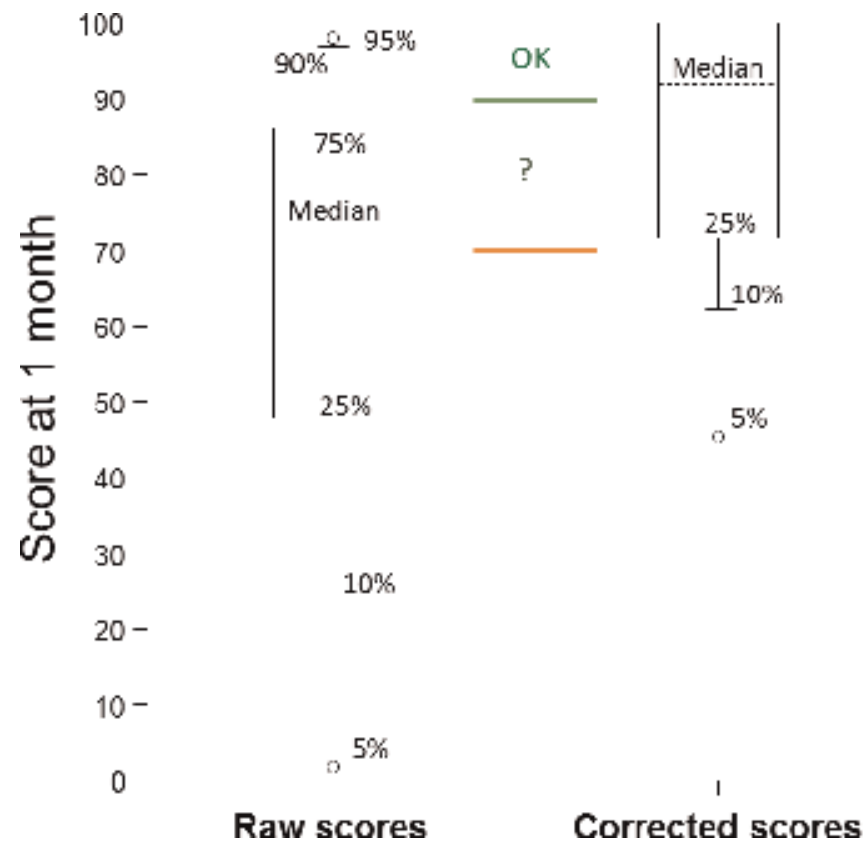

Figure 1.

Distribution of sentence recognition scores assuming correct electrode array position (raw scores) and, in addition, removing the effects of duration of deafness and etiology (corrected scores). Number labels represent percentiles for the population. The raw score distribution helps us in preoperative counseling; the corrected scores help us in remediation 1-month post-activation.

score at 70. If the result of the prediction is less than 70, the expectation is that the new user will require active rehabilitation in addition to the conventional recommendations of patient-directed listening activities.

The information gained from the model helps in setting realistic expectations during pre-implant counseling and in early planning by clinical specialists for potential rehabilitation needs. One could be advised that the challenges of adapting to the new sensations may be slow, requiring many visits not only for device fitting but also for direct practice. On the other hand, others might be advised that they may experience a rapid adaptation and likely understand most of what people say if listening in quiet circumstances. Early advice about whether to expect slow or rapid progress can also serve as a motivational tool. If new CI listeners understand how involved they will need to be once their $\mathrm{CI}$ is activated, they can be motivated to engage in listening activities as opposed to simply expecting to be fixed. Motivation has a significant impact on adult learning [3]. It may first be established by setting appropriate expectations.

During pre-implant counseling, patients are advised that there are many factors that influence results and that these will be discovered systematically beginning at the first activation of the device and at the first-month evaluations. Indeed, although patient outcomes may turn out quite differently than expected, given that not all influencing factors can be known and that each CI user is unique, early, realistic expectations establish the foundation for accepting new sound sensations.

Our research has shown that the main factors that influence performance are related to circumstances of etiology and duration of deafness, outcomes of surgical intervention of insertion depth and dislocation, and central aspects of linguistic and neurocognitive skills [1]. The variability seen in speech recognition scores are described in Tables 1 and 2 . 


\begin{tabular}{lcc}
\hline & In quiet & In noise (10 dB SNR) \\
\hline Etiology & $0.34^{* * *}$ & $0.25^{* *}$ \\
\hline Duration of deafness per year & $0.06^{*}$ & $0.08^{* *}$ \\
\hline Total in percent & $40 \%$ & $33 \%$ \\
\hline${ }^{*} p<0.05,{ }^{* *} p<0.01,{ }^{* * *} p<0.001$. & & \\
\hline
\end{tabular}

Table 1.

Patient history factors explaining significant variance $\left(^{*}\right)$ at 1-month post-activation with respect to outcomes of sentence recognition.

\begin{tabular}{lcc}
\hline & In quiet & In noise (10 dB SNR) \\
\hline Proportion of electrodes in the scala media & $0.14^{* *}$ & $0.13^{* *}$ \\
\hline Insertion length per degree & $0.09^{* * *}$ & $0.08^{* * *}$ \\
\hline Total in percent & $23 \%$ & $21 \%$ \\
\hline${ }^{* *} p<0.01,{ }^{* * *} p<0.001$. & & \\
\hline
\end{tabular}

Table 2.

Surgical factors explaining significant variance $\left({ }^{*}\right)$ at 1-month post-activation with respect to outcomes of sentence recognition.

\subsection{Main factors influencing performance}

A thorough patient history is needed to gain details of etiology and duration of hearing loss. Our studies indicate that $6-12 \%$ of the total variance for speech understanding in quiet is related to the duration of deafness and approximately $30 \%$ is related to the etiology [1]. For instance, congenital HL produces significantly poorer scores in the short term and chronic otitis media in the long term $[1,4]$. Certain diseases may produce greater damage to the cochlea resulting in poorer signal transmission after implantation such as bony tissue growth induced by meningitis or trauma. Speech signals may be distorted more than expected by poor neural representation of speech features due to anatomical distortions from diseases that affected the hearing [5]. The challenge is that characteristics of even a known etiology may not be clear.

Details concerning the duration of deafness may be elusive; for instance, defining the specific onset of significant hearing loss may be difficult to determine and impacted by hearing aid use (i.e., how much was one or two hearing aids actually used (e.g., [6]), was the loss progressive, how rapid did the loss develop, and so forth). The impact of unanswered questions may be seen in later performance, especially in cases of unexpected poor performance. Applying the predictive model helps estimate potential outcomes.

\section{Surgical intervention}

The physiological/structural condition of the cochlea may affect electrical stimulation. A full battery of objective measures available to the surgical team conducted pre- and intraoperatively helps guide preoperative planning and postoperative device programming [7]. Aspects to consider are the size of the cochlea [8], the type of electrode design (straight or perimodiolar) and its potential insertion depth, as well as whether the insertion was solely into the scala tympani or dislocated into the scala vestibuli. In our study, scala dislocation reduced scores by 12-25 points at the 1-month evaluation interval [1]. Thus, selection of the implant device, in cooperation with the patient wishes, anatomical considerations and 
surgical intervention each play a role in performance outcomes and account for $8-13 \%$ of the variance in performance scores at 1 year.

Preoperatively, it is essential to choose the appropriate electrode type and to target an insertion depth of one cochlear turn (i.e., $\sim 360^{\circ}$ ) as proposed by [1]. This aim is also supported by [9], who indicated a negative correlation between word scores and electrode insertion depth measures. The study by Lazard et al. [6] also found poorer outcomes for the most deeply inserted electrodes. These results need to be tempered against the potential of having larger frequency-place mismatches for shallower electrode insertion depths as discussed in the following section.

Any information that contributes to the first activation and mapping for listening programs is useful. The insertion depth provides a reference for better accessing appropriate frequency allocations relative to cochlear tonotopic organization [8]. Electrode design also plays a role not only because of its insertion characteristics, straight or curved, but also because of the spacing between contact electrodes.

Our studies have shown that an insertion depth of 300-360 yielded optimal performance. Moderate shifts in frequency-to-place may easily be accommodated by the listener, but larger shifts $>1.5$ octave may affect auditory performance, and adaptation may take longer [10]. Electrode placement can be detected by routine intraoperative X-ray. Shifts were approximately one octave for Nucleus Implants with $360^{\circ}$ insertion depth, with shifts still $<1.5$ octaves for $300^{\circ}$, for the default frequency allocation table. For other devices, the shifts appeared greater for the same insertion depths due to the specific default frequency-to-electrode allocation used in the device. Thus, these devices may work most effectively with greater insertion depths or, alternatively, with the use of customized frequency allocation tables that can be adjusted in the specific programming software.

Avoiding a frequency-place shift of greater than 1.5 octaves will probably produce the best result for a given insertion depth. However, further optimization may be achieved by limiting insertion depth at surgery or deactivating the most apical electrodes (e.g., [11]). If electrode arrays are found to be inserted greater than one turn, we may consider deactivating the most apical electrode contacts to simulate the ideal insertion depth. This is consistent with the work of [8] whose temporal bone studies found correlations between specific insertion depth angles and tonotopic frequency locations. Deeper insertion, greater than $360^{\circ}$, was associated with frequencies lower than $\sim 900 \mathrm{~Hz}$; however, one needs to consider that the spatial density of spiral ganglion cells increases considerably past this point, such that cross-turn stimulation can easily occur. As mentioned, depending on the device type, if the active insertion depth is limited to $360^{\circ}$, then it may be necessary to modify the frequency-to-electrode allocation through programming to avoid excessive frequency-place shifts.

\subsection{Intraoperative tests}

After the electrode has successfully been placed into the cochlea, monitoring its position is accomplished through intraoperative X-ray [7]. The neural activity of device-activated electrical stimulation is evaluated with neural response telemetry (NRT), which replicates electrically evoked compound action potentials (ECAP). The NRT responses provide an objective measure of the integrity of auditory nerve function when stimulated through a CI $[12,13]$. It can be administered intra- and postoperatively; a thorough description of the method is described by [14], and the newer application of auto-NRT is described by [15]. Intraoperatively, the focus is on gaining details relating to whether the device is operational and whether the responses per electrode indicate that electrodes are within the scala tympani and 
close enough to activate auditory nerves. Those outside, mislocated into the scala vestibuli, may yield no NRT response [13].

\section{Device activation}

It is our practice to provide two initial programs. The first is a standard, default program recommended by the manufacturer's specifications, and the second has frequency-to-electrode allocation, as indicated above, based on the surgical outcome indicated by X-ray findings for the particular patient. We ask the new user to switch between the two programs in order to experience whether one is more pleasing and/or effective than the other. We speculate that postlinguistically deafened adults will have difficulty adjusting to the sound quality for the standard program and choose the second that was derived from the intraoperative findings and one that avoids a "boomy" sound indicative of a mixing up of low-frequency sensations produced by apical cross-turn stimulation.

The second program will usually take into consideration the possible frequencyplace mismatches relative to insertion depth, that is, the physical position of the electrode contacts. The default frequency allocations provided in the programs of the sound processor may need adaptation [1], as discussed above.

All new users are sent home for a month after receiving counseling about ways in which they can direct their own rehabilitation through practice at home and in different environments in which they commonly find themselves. They are also reminded of expectations, and family members and/or significant others are provided counseling in ways to support the new CI user. It is an option to test the subject for sentence understanding in quiet during the first days of activation. We have found that if a new user scores $>60 \%$ at day 1 , they will obtain scores $>80 \%$ by the 1-month follow-up (Figure 2). These CI users will likely need little active rehabilitation and already appear to be on a good course. Thus, early performance is indicative of later, long-term performance.

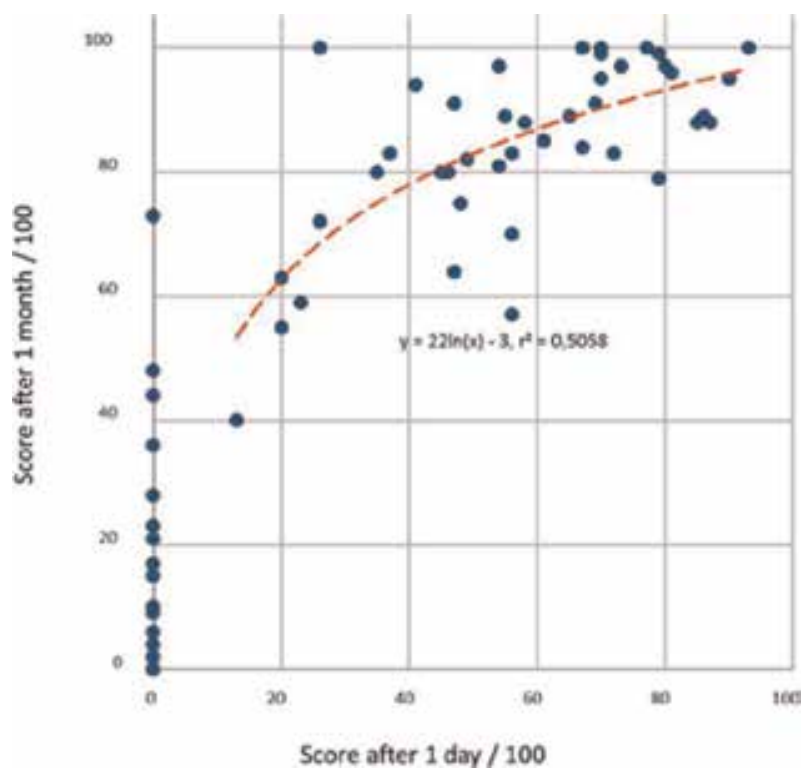

Figure 2

Sentences in quiet evaluated at 1 day and at 1 month, post-implant. 
In fact, the development of speech understanding with a CI does not follow a linear function with time. High sentence recognition scores can be obtained at only 1 day after activation, and the first 2 weeks are as important as the next 6 months and the following 2-3 years. It is not fully understood why CI user's individual performance progress at different rates. In James et al. [1], they observed different patterns of growth in scores, both in quiet and in noise, from the first month, but always following a logarithmic growth curve, such that each additional increment in performance took twice as long as the preceding increase.

\section{Optimizing maps and initial evaluations: 1-month follow-up}

Significant improvement will usually take place from activation to 1 month; thereafter increases continue but at a much slower pace. Increases in understanding will be about the same after 6 months of experience for sentences in quiet. Adapting to any new sensation requires time; an auditory signal presented through a CI will always first be perceived as very different. It is unclear why some new users immediately accept the new input and others reject it as sounding too foreign. In any case, we believe a month of exposure to the new signals is the minimum time to allow all patients for the initial accommodation to the input. Thus, all CI users are re-evaluated at 1 month.

By the first month, there already is access to data logging to confirm speech processor program usage, the users are usually aware of which program they might prefer, and the speech recognition scores in quiet will have been tested. The outcome of sentence recognition testing and CI user reports may indicate a need for alternative device programming. Looking at Tables 1 and 2, approximately 40-50\% of the variance is not explained by the patient-related and surgical factors. There are dynamics in play that may never be known such as the impact of certain disadvantages (insertion depth, dislocation, cochlear condition at surgery) and others.

Alternative programs (differing mapping parameters) may also take into consideration speed of stimulation (refractory period) as demonstrated through different stimulation rates or spread of excitation via channel selectivity (perhaps deactivating particular electrodes). These more advanced aspects of programing, however, are taken into consideration at every programming session, as indicated. Optimizing sound processor programs is the most direct way to compensate for the degraded speech signals delivered through a cochlear implant.

The one aspect to be evaluated may be behavioral responses to changes in stimulation rate. Postoperative NRT testing may be indicated to assess neural recovery functions to gain information about beneficial stimulation rates. From their studies on the temporal characteristics of auditory nerve stimulation via CIs, [16] suggest that the programmed stimulation rate relates to the refractory period of the nerve. CI user performance may be addressed, in some cases, by reducing the stimulation rate. It is not possible to define when the so-called aging process begins, but it is clear that neural transmission times slow as one ages $[17,18]$. Older CI users may be more susceptible to stimulation rate effects. Any means of enhancing auditory signals that occur in the presence of poor temporal processing will provide a better foundation for learning to overcome perceptual difficulties.

\subsection{Initial performance evaluations}

During this test interval, it is possible to identify, with more clarity, the individuals who might be classified as potentially having poor performance. By definition, on average, approximately $50 \%$ of recipients will demonstrate "normal" performance, i.e., $70 \%$ or greater scores for sentence understanding in quiet. However, if 
we consider individuals who present with no negative patient-related factors, they should perform better than $70 \%$ and on average around $90 \%$. This is, then, the second use of the model. The prediction of the model is compared with the actual score at 1-month post-activation; if the actual score is lower than the prediction, it points to a need for remedial action. Thus, two groups are identified who will undergo further evaluation: those individuals who are overall "poor" performers and achieve less than $70 \%$ and those whose actual scores are below their predicted scores from the model. The others with satisfactory performance will be advised to continue their own patient-directed practices (passive rehabilitation). Complete remediation of the effects of duration of deafness and congenital hearing loss would result in a "corrected" distribution as shown in Figure 1, with an overall average (median) performance at about $90 \%$ and only $25 \%$ of cases performing less than $70 \%$. Such an improvement is the aim of the remedial actions described in the following sections.

Figure 3 illustrates the further needs of the less-than-satisfactory poor user or overall poor performer. The results of intraoperative NRT findings are compared, and mapping considerations are applied to create alternative programs, as described above. This is considered part of the bottom-up approach. Other, more specific analytic psychophysics may also be included in a rehabilitation program, if indicated [5].

A poor performer will require thorough auditory evaluations and cognitive testing. Given that the predictive model accounts for approximately $63 \%$ of the variance in performance, the contribution of cognitive factors must be considered. If poor performance is identified or suspected, steps are taken to investigate the factors that may be affecting the user's ability to process the sound information they are receiving through the CI including the central aspects of linguistic and neurocognitive skills influencing communication strategies as outlined in Figure 4.

Evaluations that yield scores within normal limits for phonological sensitivity and working memory point to motivation issues and, therefore, intense counseling are provided without the need for active rehabilitation support. If poor linguistic skills are revealed, training in phonological aspects is indicated. Evaluations

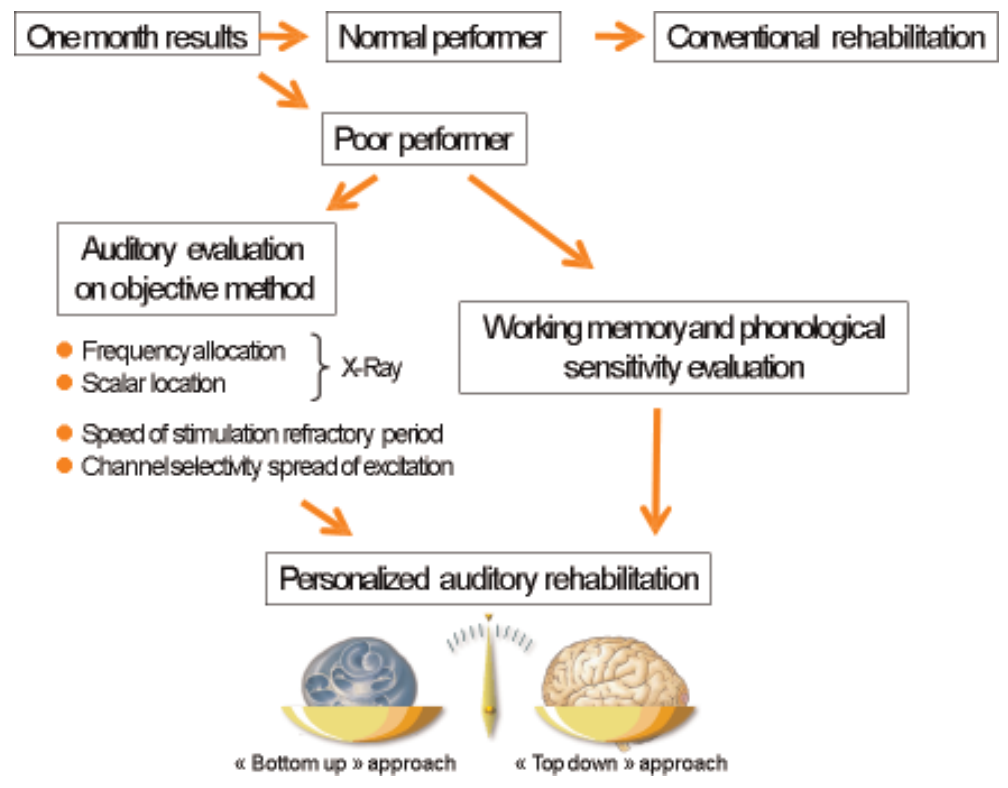

Figure 3.

Flow diagram illustrating the development of patient-specific rehabilitation strategies. 


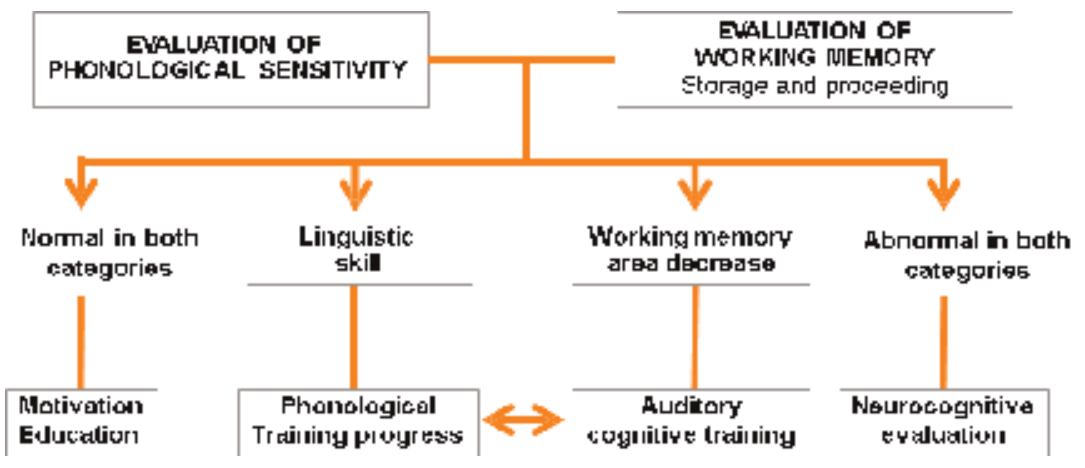

Figure 4.

Indications for rehabilitation training in poorer performers.

demonstrating poor working memory lead to applying auditory cognitive training; however, if the results of the evaluations point to an abnormal working memory and phonological sensitivity, neurocognitive evaluations are pursued.

It is beyond the scope of this chapter to supply specific evaluation and training materials. Methods should be consistent with culture and the available materials in a particular language and according to the consensus within the country. A review of rehabilitation methods that can be applied to cochlear implant users can be found in [19] and in [20]. In common, however, is that counseling will focus on the CI user gaining confidence in associating the digitally coded sounds that are presented through a CI with meaningful speech. It is advisable to remember that a CI user needs adequate time to experience modifications; even poorer users do not require constant reprogramming. In general, poorer users are seen at the clinic in 3-month intervals, and better performers are seen in 6-month to annual intervals. Interactions with local speech-language therapists are the main support for poorer performers with frequent liaison between the therapist and specialists within our clinic.

\subsection{Rehabilitation approach}

Harris et al. [2] point out that no standardized rehabilitation approach exists despite decades of CI use in individuals of all ages. Agreement is found in the concept of tailoring post-implant rehabilitation to the needs of the individual user $[21,22]$. The challenge is that long-term rehabilitation may be indicated but that limitations in funding through reimbursement are available mainly due to a lack of evidence for demonstrable effects $[2,18]$. Our experience, and that of [21], indicates that rehabilitation may be required for as long as 2 years to reach a so-called performance plateau.

Conceptually, rehabilitation can be divided into two approaches, top-down or bottom-up [23]. Methods that focus on bottom-up procedures utilize materials relating specifically to the input signals possible via a CI, that is, how a signal is processed. The elements of sound serve as building blocks, starting with the smallest unit (i.e., a phoneme). Relative to a CI, acquiring responses to the psychophysical tasks (temporal, spectral, and amplitude cues) during the mapping process entails a bottom-up approach, which is an analytic method. Some of these tasks may be adapted for auditory training purposes [5].

\subsubsection{Synthetic-cognitive training (top-down)}

Top-down methods represent a synthetic approach and have the aim of enhancing communication strategies through cognitive processing. As mentioned, 
outcomes of the predictive model accounted for more than $60 \%$ of the variance in quiet and $50 \%$ of the variance in noise for sentence recognition scores obtained at 1 month after CI activation [1]. Thus, cognitive factors play a large role in the wide variance seen in performance scores obtained by the adult CI population. Optimizing a personalized rehabilitation strategy must take into consideration the cognitive dynamics of speed of processing, working memory, and attention and executive function [23].

The input from any $\mathrm{CI}$ is inherently degraded compared to that available in normal-hearing individuals or, indeed, to those able to utilize a hearing aid effectively. Aging may play a role, slowing the process of learning [18] to accommodate to speech sounds presented as a new, seemingly unusual, set of sounds. Cognitive training should take into consideration the age of the CI user. In fact, it has been suggested that older CI users ( $>80$ years) may benefit more from rehabilitation than younger users. A top-down approach may be the most appropriate approach for the older population [18].

\subsection{Role of plasticity}

It is unknown to what degree the brain reorganizes speech when confronted with hearing loss [24]. We studied the dynamics of reversed cross-modal plasticity by TEP brain imaging during speech tracking before and after CI at two time points [25]. Essentially, as a result of auditory sensory deprivation, regions in the brain associated with perceiving visual input are activated during speech communication. After implantation, neuroplasticity is demonstrated as the brain recruits more auditory networks during tests of speech recognition. Olds et al. [26] confirmed these findings using the functional near-infrared spectroscopy (fNIRS) imaging technique. They observed cortical reorganization and suggested that listening effort may be involved in the cortically activated regions. They used several speech recognition tests, including sentences, with the CI turned off and on. This may account for the activated regions seen in our study, although neither of the test intervals utilized direct auditory input. We speculate that during hearing deprivation, sensitivity to voice progressively decreases. Anderson and Kraus [20] refer to this as "deprivation-induced changes in auditory mapping." Once sound is reintroduced, the more visually focused cortical regions reassert into the voice-sensitive regions. This cross-modal reactivation shows the cooperation between visual and auditory cortex. Thus, a profound aim of active rehabilitation is to take advantage, and encourage, reverse plasticity to aid in restoring cortical preference to meaningful auditory signals. This need is also recognized by other authors [27].

\section{Ongoing post-implant support, evaluations of progress, and hearing training: 6-month follow-up}

Testing speech in noise takes place at 6 months. We have seen that the relationship between performance in quiet and in noise is highly correlated. The early performance in quiet is manifested in the 6-month scores (see Figure 1). Testing at a signal-to-noise ratio (SNR) of $10 \mathrm{~dB}$ creates a reasonable challenge and serves as a further indicator of who requires continued rehabilitation. We have observed that it is possible to identify CI users who have demonstrated early success or a steep learning curve. The remaining patients continue as poor users needing support and ongoing counseling to maintain their motivation. With continued exposure to auditory stimulation, they can be advised that still more progress is possible for them. 
Increases continue even up to 3 years, but the incremental gain is much less that what is usually seen during the first 6 months of use.

\section{Summary}

We summarize the complete rehabilitation process in Figure 5. Pre-implant counseling based on the results of the predictive modeling; surgical planning focusing on considerations to the size of cochlea and type of electrode; and intraoperative testing using X-ray findings to confirm placement and depth of insertion along with NRT to confirm neural interface via electrostimulation all take place before initial activation. This includes counseling that may need to modify expectations based on surgical outcomes and intraoperative evaluations. Two MAPs are developed at first fitting where one is based on intraoperative findings. At 1 month, observations gathered from data logging, along with comparing sentence scores in quiet to the predictive model, provide an indication as to whether a new CI user will need specialized rehabilitation. Again, counseling may need to guide and modify expectations. The type of rehabilitation is determined, usually a combination of both bottom-up and top-down approaches. At the 6-month interval, testing in noise is applied, and further adaptations to the MAPs are made. In the future, we hope to extend the predictive model to include factors for analysis of performance in noise for the long term. Continued appropriate rehabilitation after 6 months ensues, and continued counseling insures that the CI user understands the need to support hearing progress with ongoing rehabilitation, if needed.

Providing viable rehabilitation to adult poor performers lies within the realm of detective work. In the early stages, it provides affirmative counseling based on predictive modeling and effective surgical planning and its implementation. Counseling patients with realistic expectations, however, takes place throughout the entire rehabilitation process. There will always be differences in outcomes, but having a full array of options based on objective measures and individual case history will guide the specialist to advise for optimal use of their hearing abilities. Motivation is a very important component of success, and this needs to be reinforced especially for this population who, often, have unrealistic expectations (this includes the family and supporting individuals).

As CI specialists, we provide access to direct rehabilitation and rehabilitation support. Specialized rehabilitation, given the wide variability in patient outcomes,

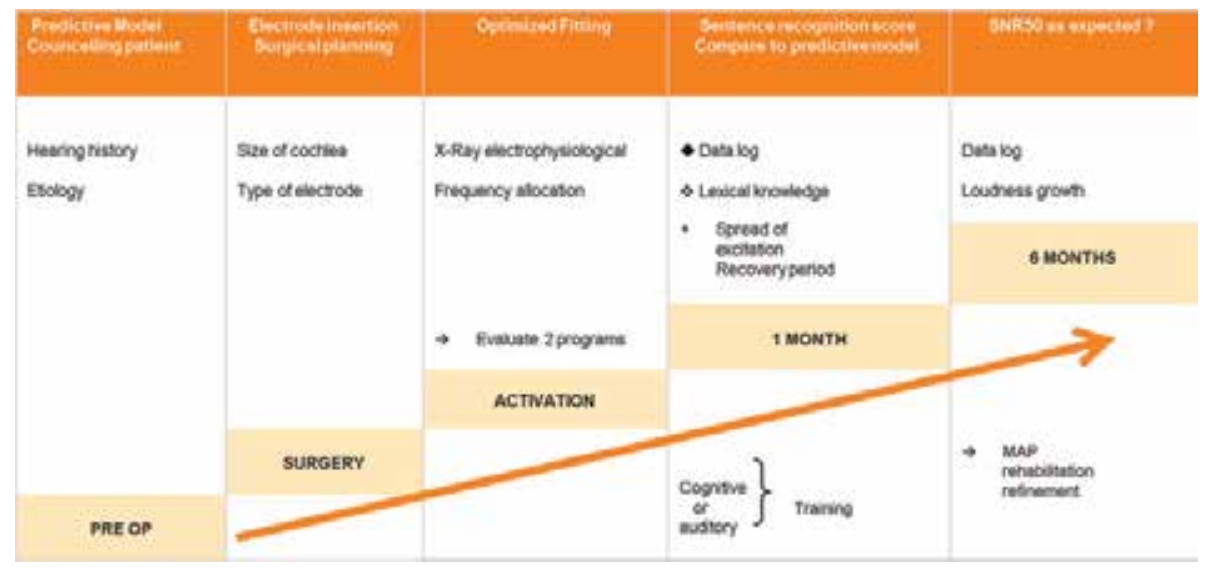

Figure 5.

Stages of the rehabilitation process. 
ideally should be modeled to the specific needs of each individual CI user. To achieve the best level of performance possible, programming options will continually be investigated, supported by patient-directed auditory experience and phonologic and cognitive training, when necessary.

We have discussed only the factors that may influence the post-implant performance of adult poor users, giving guidance on how best to examine the factors that affect performance. Our responsibility as clinicians is to offer an adult patient-user guidance that leads to an improvement in their quality of life through better hearing. We aim to utilize professional time efficiently and effectively, and we aim to concentrate on those who need post-implant therapy rather than providing standard rehabilitation strategies that may miss some and waste time for others.

\section{Acknowledgements}

We would like to thank the speech therapists in our service for their considerable input to the rehabilitation process. We would like to thank Ms. Dianne Mecklenburg for editing this chapter.

\section{Declaration}

Author CJJ is an employee of Cochlear, manufacturer of cochlear implants.

\section{Author details}

Bernard Fraysse $^{1 *}$ and Chris J. James ${ }^{2}$

1 Hôpital Purpan, CHU Toulouse, France

2 Cochlear France SAS, Toulouse, France

*Address all correspondence to: fraysse.sec@chu-toulouse.fr

\section{IntechOpen}

(C) 2019 The Author(s). Licensee IntechOpen. This chapter is distributed under the terms of the Creative Commons Attribution License (http://creativecommons.org/licenses/ by/3.0), which permits unrestricted use, distribution, and reproduction in any medium, provided the original work is properly cited. (c) BY 


\section{References}

[1] James C, Karoui C, Laborde M-L, Lepage B, Molinier CE, Tartayr M, et al. Early sentence recognition in adult cochlear implant users. Ear and Hearing. 2019;40;905-917. DOI: 10.1097/ AUD.0000000000000670

[2] Harris MS, Capretta NR, Henning SC, Feeney L, Pitt MA, Moberly AC. Postoperative rehabilitation strategies used by adults with Cochlear implants: A pilot study. Laryngoscope Investigative Otolaryngology. 2016;1(3):42-48

[3] Dirbashi A: The Role of Motivation in Adult Education. 2017. Available from: www.linkedin.com/pulse/role-motiva tion-adult-education-abdulrahman-al-d irbashi [Accessed: 20 October 2018]

[4] Blamey P, Artieres F, Başkent D, Bergeron F, Beynon A, Burke E, et al. Factors affecting auditory performance of postlinguistically deaf adults using Cochlear implants: An update with 2251 patients. Audiology and Neurotology. 2013;18:36-47

[5] Garadat SN, Zwolan TA, Pfingst BE. Using temporal modulation sensitivity to select stimulation sites for processor MAPs in cochlear implant listeners. Audiology \& Neuro-Otology. 2013; 18(4):247-260

[6] Lazard DS, Vincent C, Venail F, Van de Heyning P, Truy E, Sterkers O, et al. Pre-, per- and postoperative factors affecting performance of postlinguistically deaf adults using cochlear implants: A new conceptual model over time. PLoS One. 2012;7(11): e48739

[7] Vickers D, Said S. The role of objective measures and imaging to optimise cochlear implant outcomes. ENT \& Audiology News. 2017;4. Available from: www.entandaudiolog ynews.com
[8] Stakhovskaya O, Sridhar D, Bonham BH, Leake PA. Frequency map for the human cochlear spiral ganglion: Implications for cochlear implants. Journal of the Association for Research in Otolaryngology. 2007;8(2):220-233

[9] Holden LK, Finley CC, Firszt JB, Holden TA, Brenner C, Potts LG, et al. Factors affecting open-set word recognition in adults with cochlear implants. Ear and Hearing. 2013;34(3): 342-360

[10] Li T, Galvin 3rd JJ, Fu QJ. Interactions between unsupervised learning and the degree of spectral mismatch on short-term perceptual adaptation to spectrally shifted speech. Ear and Hearing. 2009;30:238-249

[11] Gani M, Valentini G, Sigrist A, Kós M-I, Boëx C. Implications of deep electrode insertion on cochlear implant fitting. Journal of the Association for Research in Otolaryngology. 2007;8(1): 69-83

[12] Christov F, Munder P, Berg L, Arnold J, Bagus H, Lang S, et al. A twelve months follow-up: Influence of origin and duration of hearing-loss on Tnrts after Cochlear implantation. Otolaryngology. 2016;6:258

[13] Lenarz T. Cochlear implant-State of the art. GMS Current Topics in Otorhinolaryngology-Head and Neck Surgery. 2018;16:Doc04

[14] Abbas PJ, Brown CJ, Hughes ML, Gantz BJ, Wolaver AA, Gervais JP, et al. Electrically evoked compound action potentials recorded from subjects who use the nucleus CI24M device. The Annals of Otology, Rhinology \& Laryngology. Supplement. 2000;185:6-9

[15] Tanamati LF, Muniz LF, Samuel PA, Goffi-Gomez MVS, Wiemes GRM, 
Lima DP, et al. Use of remote control in the intraoperative telemetry of cochlear implant: Multicentric study.

Brazilian Journal of Otorhinolaryngology. 2018;85(4):502-509. pii:

S1808-8694(18)30227-1

[16] Hughes ML, Laurello SA. Effect of stimulus level on the temporal response properties of the auditory nerve in cochlear implants. Hearing Research. 2017;351:116-129

[17] Anderson S, Parbery-Clark A, White-Schwoch T, Kraus N. Aging affects neural precision of speech encoding. The Journal of Neuroscience. 2012;32(41):14156-14164

[18] Armero O, Hicks C. Aural rehabilitation for older adults. Hearing Review. 2018;25(5):12-16

[19] Sweetow RW, Sabes JH.

Technologic advances in aural rehabilitation: Applications and innovative methods of service delivery. Trends in Amplification. 2007;11(2): 101-111

[20] Anderson S, Kraus N. Auditory training: Evidence for neural plasticity in older adults. Perspectives on Hearing and Hearing Disorders. Research and Research Diagnostics. 2013;17:37-57

[21] Moberly AC, Bates C, Harris MS, Pisoni DB. The enigma of poor performance by adults with Cochlear implants. Otology \& Neurotology. 2016; 37(10):1522-1528

[22] Pichora-Fuller MK. Cognitive aging and auditory information processing. International Journal of Audiology. 2003;42(suppl 2):26-32

[23] Moberly AC, Harris MS, Boyce L, Nittrouer S. Speech recognition in adults with Cochlear implants: The effects of working memory, phonological sensitivity, and aging. JSLGR. 2017;60: 1046-1061
[24] Lazard DS, Giraud AL. Faster phonological processing and right occipito-temporal coupling in deaf adults signal poor cochlear implant outcome. Nature Communications. 2017;8:14872. 2012;7(11):e48739

[25] Rouger J, Lagleyre S, Démonet JF, Fraysse B, Deguine O, Barone P.

Evolution of crossmodal reorganization of the voice area in cochlear-implanted deaf patients. Human Brain Mapping. 2012;33(8):1929-1940

[26] Olds C, Pollonini L, Abaya H, Larky J, Loy M, Bortfeld H, et al. Cortical activation patterns correlate with speech understanding after Cochlear implantation. Ear and Hearing. 2016;37(3):e160-e172

[27] Kramer S, Vasil KJ, Adunka OF, Pisoni DB, Moberly AC. Cognitive functions in adult Cochlear implant users, Cochlear implant candidates, and normal-hearing listeners. Laryngoscope Investigative Otolaryngology. 2018; 3(4):304-310 


\title{
Cochlear Implant in Single-Sided Deafness Children and Adults
}

\author{
Ángel Ramos Macías, Silvia A. Borkoski Barreiro, \\ Juan Carlos Falcón González and Ángel Ramos de Miguel
}

\begin{abstract}
Binaural hearing in humans ensures our effective communication as it improves the sound localization, the speech understanding in noise, the spatial awareness, the listening easiness, and the development of spoken language. The majority of the literature on patients with single-sided deafness and cochlear implantation involves adult patients; the cochlear implant is a viable and cost-effective treatment option for this population. So in this chapter, we will emphasize the importance of early treatment of unilateral hearing loss in the pediatric age and the emerging indication of the cochlear implant as a treatment.
\end{abstract}

Keywords: cochlear implant, single-sided deafness, unilateral hearing loss, tinnitus, children, quality of life, language

\section{Introduction}

Binaural hearing in humans ensures our effective communication as it improves the sound localization, the speech understanding in noise, the spatial awareness, the listening easiness, and the development of spoken language. Lack of binaural input and diminished audibility negatively affects the abovementioned factors, thereby affecting communication and quality of life [1-3].

The term single-sided deafness (SSD) is defined by the presence of a complete hearing loss in one of the two ears, and thresholds better than $25 \mathrm{~dB}$ at all frequencies, in contralateral side.

During the human development, binaural hearing is even more important in young children than in adult. The neural circuit for binaural processing is innate and functional at birth, so the neural consequences of lack of binaural input and diminished audibility should not be underestimated. The asymmetric development promoted by unilateral implant did not eliminate integration of binaural input in the brainstem, but severe impairments were found in perception of binaural cues. Given that binaural processing, at least, at the brainstem, is possible, it can be hypothesized that perception of binaural cues would be established in children receiving bilateral cochlear implants with long-term use but that differences from normal would persist with increasing abnormalities for those children who had experienced longer durations of unilateral implant use [4].

Although the importance of binaural hearing was demonstrated several decades ago, the treatment of SSD has only become an increased focus of attention since the beginning of the last decade [5-8]. 
There is a paucity of high-level evidence with many studies reporting with cochlear implants that have been used in adult patients with unilateral, severeprofound sensorineural hearing loss with associated disabling tinnitus and normal contralateral hearing with good results [9-15].

While cochlear implant (CI) provision is a well-established and beneficial therapy in an increasing number of countries for adults with acquired SSD, there is less experience with this therapeutic option in children [16-19].

The estimated incidence of sensorineural hearing impairment ( $>40 \mathrm{~dB} \mathrm{HL})$ at birth is 1.86 per 1000 newborns in developed countries and $30-40 \%$ of these are unilateral. The prevalence of unilateral hearing loss (UHL) increases with age due to cases of delayed onset before 5 years. At school age, up to 3-6\% of children with different grades of UHL could be found [20-22].

\section{Consequences of SSD in children}

There is evidence that single-sided deafness negatively impacts on the development of children, not only presenting difficulties in locating sounds and understanding speech in noise but also having difficulty in mastering complex skills for spoken language which can cause significant psychosocial difficulties and school problems. Children suffering from SSD that receive a CI have the potential to obtain useful information from an ear that, without the implantation, would contribute poorly or not contribute, thus, increasing the quality of their general communication $[23,24]$.

\subsection{Speech and language}

Any degree of hearing loss makes children at risk of producing shorter expressions and being less able to produce verbs related to grammatical morphology. Studies show that children and adults with SSD have worse results in speech recognition in noise and have fewer skills for sound localization [21-25].

Sangen et al. were among the first to study language skills in children with SSD. The study showed differences between children with SSD and normal-hearing children on several language skills (morphology, syntax, and vocabulary tests) and on auditory behavior, presumably due to auditory input interrupted during the time of language acquisition [26].

Difficulties in language oral and reading skills and the worst results in the recognition of words and language, although improving over time, it has been observed that adolescents with SSD continue to present worse results in language tests compared to normal hearing [27].

Ramos et al. in their study show that cochlear implant provides children with congenital SSD with significant audiological and subjective benefits. Children with congenital SSD and implanted after a long hearing deprivation period ( $>6$ years) may not have an important binaural benefit, although bilateral effect can be achieved. Children with post-lingual unilateral deafness and after a short period of hearing deprivation probably integrated the normal acoustic hearing with the cochlear implant electrical signal and showed binaural benefits (Figure 1) [19].

Studies performed on children with single-sided deafness have shown that the postoperative hearing benefit was significant in the auditory tests for understanding speech in noise. Most of the studies performed on CI recipients suffering from acquired SSD demonstrate during the postoperative period a significant decrease in location error in the implanted ear [19, 28, 29]. 


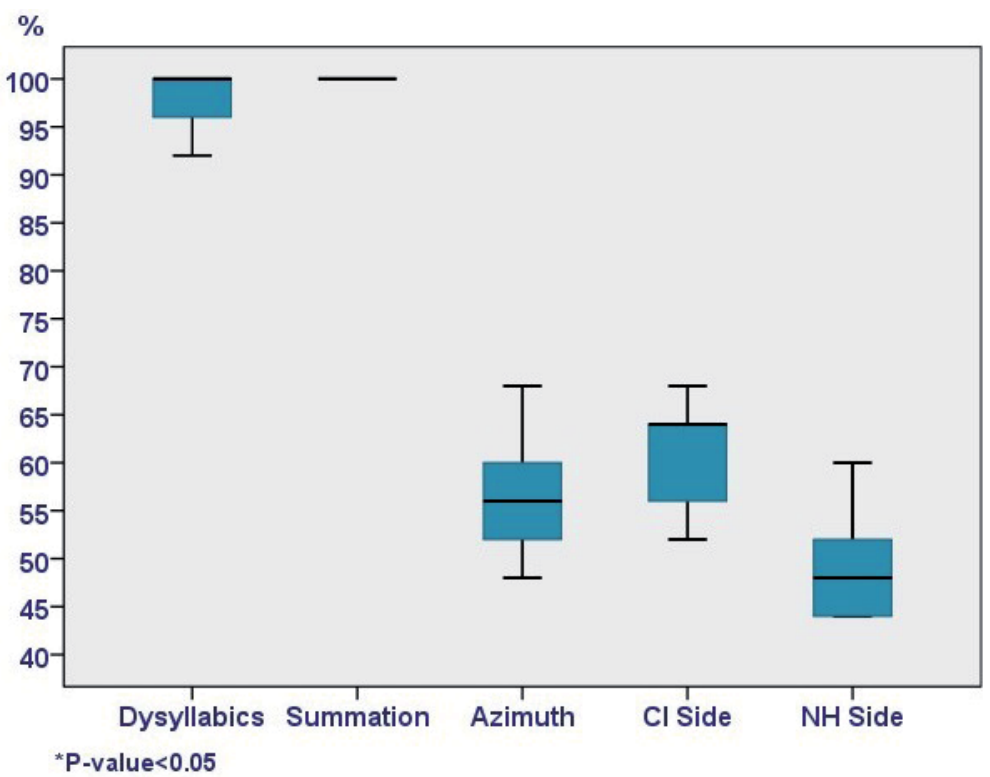

Figure 1.

Ramos et al. acquired SSD: speech test results [19].

Early intervention could prevent such language difficulties and minimize problems with spatial hearing and speech understanding. All the children's parents in this study confirmed their choice of using a CI. This established a high score in the CI satisfaction parameter by the parents regardless of the age of implantation [19].

\subsection{Cognitive functions and neural processing}

Unilateral hearing losses in children have traditionally been underappreciated. The common wisdom among medical and educational professionals has been that at least one normal-hearing or near-normal-hearing ear was sufficient for typical speech and language development in children. Different patterns of plasticity occur following partial and profound unilateral deafness, and if it is of congenital etiology, it poses severe challenges for the maturation of the brain [30, 31].

It has been reported both in adults and children with SSD that changes in the auditory cortex and other brain structures as a result of an imbalance in the auditory input have a cascading effect with worsening in neural networks and processes involved in executive function, cognition, attention, and understanding of language [4, 21, 30, 32].

The study of Vila et al. shows significant differences in how sound is processed in the cortex in children with SSD compared to normal-hearing children with functional magnetic resonance studies (fMRI) [33].

\subsection{School performance and quality of life}

Although children with SSD often function satisfactorily at an early age, many experience difficulties in school; $22-59 \%$ experience increased fatigue due to the additional cognitive effort devoted to detecting, decoding, processing, and understanding, which translates most of the time into worse school performance [21, 34, 35].

Studies reveal a decrease in the quality of life in children with SSD in different domains especially in school activities and social interactions, which are especially important for development and learning. Problems in the field of social and emotional behavior continue even in adulthood $[2,27]$. 
Close monitoring and good communication between professionals in different domains are crucial in order to minimize the potential negative effects of UHL.

Although considerable work has been done on the quality of life (QoL) attainment and health economic implications of cochlear implants, further studies are needed to characterize the costs and benefits with respect to the recipients' health, well-being, and contributions to society in cases of unilateral hearing patients.

\section{Etiology, diagnosis, and treatment}

In bilateral hearing loss, the genetic cause is the most frequent, whereas in SSD it does not occur with the same incidence. Genetic mutations occur in $28 \%$ of cases [35]. Although some syndromes (brachi-oto-renal syndrome, Waardenburg) may initially present as SSD, they usually progress toward bilateral hearing loss. Between 35 and $64 \%$ of the cases, the UHL is of unknown cause. Hearing loss is progressive in 14 and $32.8 \%$, and this is frequently associated with malformations in the inner ear. Aplasia or hypoplasia of the cochlear nerve occurs more frequently in SSD, up to $50 \%$. Among the postnatal causes, cytomegalovirus (CMV) infection, cranioencephalic trauma, and meningitis are the most frequent causes. It is also important to keep in mind the external and middle ear problems as a probable cause of an HU [36].

Thanks to vaccination schedules, it is rare to find deafness secondary to parotiditis, measles, or rubella, although anti-vaccine currents increase the risk of these old diseases. It is important to always verify during the anamnesis the correct vaccination of children with sensorineural hearing loss.

The criteria for candidacy for SSD cochlear implantation are emerging. Children with unilateral deafness or asymmetric hearing loss who have traditionally not been considered candidates for cochlear implantation should be evaluated individually. The audiological management of these potential candidates is not very different compared to children who are recipients of traditional cochlear implants. The diagnosis requires the exhaustive collection of family and personal history, including risk factors and a detailed physical examination, as well as the realization, when necessary, of the relevant complementary studies (genetic tests, imaging tests, laboratory tests, and other complementary explorations (e.g., EKG)). In the study of the etiology of neonatal hearing loss, it is important to perform a radiological study using computerized tomography and/or magnetic resonance, each of which provides different characteristics for the study of the different anatomical, pathological alterations in the external ear, medium, and internal, as well as in the central auditory pathways. Like any other intervention in medicine, the treatment must be individualized taking into account the characteristics, needs, and expectations of each child and their family (Figure 2) [36, 37].

Achieving a binaural hearing and maintaining the auditory pathway of the affected side, avoiding brain reorganization and its consequences, are the main reasons for performing a cochlear implant in a unilateral sensorineural hearing loss of cochlear origin.

In children with SSD who systematically use their CI, we know that bimodal stimulation does not generate any conflict in the auditory integration, nor does it cause the use of an implant in the affected ear while having normal hearing in the other $[38,39]$.

Children with unilateral CI for SSD need special rehabilitation method by using masking of the normal-hearing ear.

Children with acquired SSD are likely to gain similar positive benefits from cochlear implantation as those recently reported in adults (improved localization and better speech understanding in specific noise conditions). However, implantation of children with prelingual UHL is currently problematic as the impact of UHL may not become apparent until the child enters full-time education, by which time 


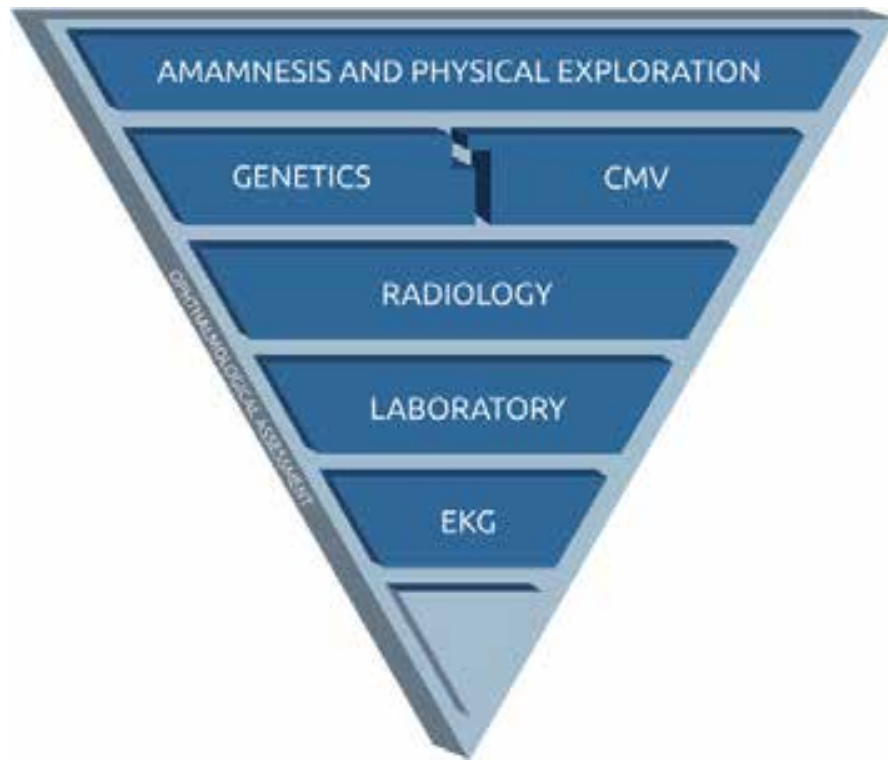

Figure 2.

Recommended sequence for etiological diagnosis (levels of diagnostic yield, ordered from highest to lowest) [35].

outcomes from cochlear implantation may be suboptimal due to auditory deprivation. In congenital sensorineural UHL, the delay in cochlear implantation may produce nonoptimal results [37].

Special consideration should be given to those unilateral congenital neurosensory hearing loss secondary to CMV and to malformations of the inner ear if these exist in both ears, given the high probability of loss of the contralateral healthy ear $[19,37]$.

\section{What is amblyaudia?}

The term amblyaudia describes persistent auditory difficulties in individuals with a history of UHL during the critical period of brain development [37].

Early exposure to sound allows adequate development and maturation of auditory processing centers. The development of the process we know as hearing and perception requires a binaural auditory ability, which makes elements such as redundancy and the shadow effect of the head, suppression, and masking (cocktail party effect) come into play to allow locating sound, spatial hearing, and perception of speech in noise.

The clinical presentation and long-term impact of amblyaudia, being a phenomenon of recent description, are not correctly defined, but it is known that subjects at risk of amblyaudia have a hearing loss with three specific aspects: asymmetric deafness, measurable loss $>30 \mathrm{~dB}$, and the loss which occurs during the critical period of development. Its presentation may be subtle and not detected in the usual audiometric tests, so it is important to bear in mind this emergent diagnostic entity and the long-term consequences of unilateral hearing loss and asymmetric hearing loss in childhood [37].

\section{Adults with UHL and accompanying severe tinnitus treated with a cochlear implant}

Prolonged spontaneous tinnitus of some degree is experienced by up to $20 \%$ of the adult population with exact estimates varying according to the tinnitus 
definition used. Subjects with tinnitus report poor speech perception, difficulty falling asleep, difficulty concentrating, insecurity, and in many cases depression. For some people their tinnitus is persistent, debilitating, and has a negative impact on their quality of life, even when present in only one ear [38-40].

The use of a CI primarily to suppress tinnitus has been considered for those patients who have incapacitating tinnitus and a UHL and thus would not normally meet the standard criteria for CI. Studies have shown the CI has successfully been used to treat the tinnitus symptoms with the benefits lasting long term $[11,41,42]$. The CI is also able to restore true binaural input for these patients providing them with the advantages of binaural hearing for listening in noise and sound localization.

The impact of cochlear implantation on the reduction of tinnitus is wellestablished; in addition to improvements in hearing, a statistically and clinically significant reduction in the loudness and disability of tinnitus is reported. Longterm studies reporting results up to 10 years after activation also show continued reduction of tinnitus loudness to very low levels and 100\% continued device use. A hypothesis is that routine daily use of the implant leads to residual tinnitus inhibition with prolonged time constants. In some patients this residual inhibition could last overnight (i.e., the switch-off period) and occasionally provides full tinnitus inhibition during day and night as reported regularly in studies with conventional CI candidates with less burdensome tinnitus [43-46].

Patients with tinnitus and SSD feel considerably worse off than those with tinnitus alone and similarly disadvantaged to more traditional implant candidates with bilateral hearing loss. Cochlear implants can reduce or suppress incapacitating tinnitus in patients with unilateral, severe-profound sensorineural hearing loss and normal contralateral hearing. It is a valid and effective therapy when other treatments have failed. The impact on quality of life of the CI on these two groups is equivalent, and a CI should be considered as an effective treatment for this population.

The reduction of tinnitus after cochlear implantation may be due to several mechanisms, such as habituation, acoustic masking, direct stimulation of the cochlear nerve, and reorganization of cortical areas.

The result was more in favor to make CI treatment in cases with short period of tinnitus ( $<5$ years).

\section{Conclusions}

Limited audibility and atypical hearing experience affects listening skills; good contralateral hearing is not enough to promote normal auditory, linguistic, and cognitive development.

It is accepted that the majority of children with acquired unilateral hearing loss who receive CI as treatment have better performance in hearing and speech performance than those with a no cochlear implant; the lower the age of implantation, the better their performance; and congenital children must be more evaluated in more multicenter studies to clarify the long-term results.

The CI in adults with SSD and severe tinnitus is a valid and effective therapy when other treatments have failed.

\section{Conflict of interest}

None declared. 


\section{Nomenclature}

SSD

$\mathrm{dB}$

CI

UHL

fMRI

QoL

EKG

single-sided deafness

decibels

cochlear implant

unilateral hearing loss

functional magnetic resonance studies

quality of life

electrocardiogram

\section{Author details}

Ángel Ramos Macías ${ }^{1,2}$, Silvia A. Borkoski Barreiro ${ }^{1,2 *}$, Juan Carlos Falcón González ${ }^{1,2}$ and Ángel Ramos de Miguel ${ }^{1,2}$

1 Department of Otolaryngology Head Neck Surgery, Complejo Hospitalario Universitario Insular Materno Infantil de Gran Canaria, Las Palmas, Spain

2 Department of Otolaryngology, Psychoacoustic and Balance Laboratory, Las Palmas University (ULPGC), Las Palmas, Spain

*Address all correspondence to: silviaborkoski@hotmail.com

\section{IntechOpen}

(C) 2019 The Author(s). Licensee IntechOpen. This chapter is distributed under the terms of the Creative Commons Attribution License (http://creativecommons.org/licenses/ by/3.0), which permits unrestricted use, distribution, and reproduction in any medium, provided the original work is properly cited. (cc) BY 


\section{References}

[1] Jacobs E, Langereis MC, Frijns JH, Free RH, Goedegebure A, Smits C, et al. Benefits of simultaneous bilateral cochlear implantation on verbal reasoning skills in prelingually deaf children. Research in Developmental Disabilities. 2016;58:104-113

[2] Roland L, Fischer C, Tran K, Rachakonda T, Kallogjeri D, Lieu JE. Quality of life in children with hearing impairment: Systematic review and metaanalysis. Otolaryngology and Head and Neck Surgery. 2016;155(2):208-219

[3] Sladen DP, Frisch CD, Carlson ML, Driscoll CL, Torres JH, Zeitler DM. Cochlear implantation for singlesided deafness: A multicenter study. Laryngoscope. 2017;127(1):223-228

[4] Tillein J, Hubka P, Kral A. Monaural congenital deafness affects aural dominance and degrades binaural processing. Cerebral Cortex. 2016;26(4):1762-1777

[5] MacKeith NW, Coles RR. Binaural advantages in hearing of speech. The Journal of Laryngology and Otology. 1971;85:213-232

[6] Bronkhorst AW, Plomp R. Binaural speech intelligibility in noise for hearing-impaired listeners. The Journal of the Acoustical Society of America. 1989;86:1374-1383

[7] Colburn HB, Shinn-Cunningham B, Kidd G Jr, Durlach N. The perceptual consequences of binaural hearing. International Journal of Audiology. 2006;45(Suppl 1):34-44

[8] Firszt JB, Reeder RM, Skinner MW. Restoring hearing symmetry with two cochlear implants or one cochlear implant and a contralateral hearing aid. Journal of Rehabilitation Research and Development. 2008;45:749-767
[9] Buechner A, Brendel M, LesinskiSchiedat A, Wenzel G, Frohne-Buechner C, Jaeger B, et al. Cochlear implantation in unilateral deaf subjects associated with ipsilateral tinnitus. Otology \& Neurotology. 2010;31:1381-1385

[10] Masgoret E, Meran J, Moreno C, Falcón JC, Artiles O, Ramos A. Acúfeno e implante coclear. Experiencia preliminar. Acta Otorrinolaringológica Española. 2010;61:405-411

[11] Ramos Á, Polo R, Masgoret E, Artiles O, Lisner I, Zaballos ML, et al. Implante coclear en pacientes con hipoacusia súbita unilateral y acúfeno asociado. Acta Otorrinolaringológica Española. 2012;63:15-20

[12] Ramos Macías A, Falcón-González JC, Manrique Rodríguez M, Morera Pérez C, García-Ibáñez L, Cenjor Español C, et al. One year results for patients with unilateral hearing loss and accompanying severe tinnitus and hyperacusis treated with a cochlear implant. Audiology \& Neuro-Otology. 2018;23(1):8-19. DOI: $10.1159 / 000488755$

[13] Holder JT, O’Connell B, HedleyWilliams A, Wanna G. Cochlear implantation for single-sided deafness and tinnitus suppression. American Journal of Otolaryngology. 2017;38:226-229

[14] Mertens G, De Bodt M, Van de Heyning P. Evaluation of long-term cochlear implant use in subjects with acquired unilateral profound hearing loss: Focus on binaural auditory outcomes. Ear and Hearing. 2017;38:117-125

[15] Mertens G, De Bodt M, Van de Heyning P. Cochlear implantation as a long-term treatment for ipsilateral incapacitating tinnitus in subjects with 
unilateral hearing loss up to 10 years. Hearing Research. 2016;331:1-6

[16] Plontke SK, Heider C, Koesling S, et al. Cochlear implantation in a child with posttraumatic single-sided deafness. European Archives of OtoRhino-Laryngology. 2013;270:1757-1761

[17] Hassepass F, Aschendorff A, Wesarg T, Kröger S, Laszig R, Beck RL, et al. Unilateral deafness in children: Audiologic and subjective assessment of hearing ability after cochlear implantation.

Otology \& Neurotology. 2013;34(1):53-60

[18] Kitoh R, Moteki H, Nishio S, Shinden S, Kanzaki S, Iwasaki S, et al. The effects of cochlear implantation in Japanese single-sided deafness patients: Five case reports. Acta OtoLaryngologica. 2016;136:460-464

[19] Ramos Macías A, Borkoski Barreiro SA, Falcón González JC, de Miguel Martínez I, Ramos de Miguel A. Single-sided deafness and cochlear implantation in congenital and acquired hearing loss in children. Clinical Otolaryngology. 2019;44(2):138-143. DOI: $10.1111 /$ coa.13245

[20] van Wieringen A, Boudewyns A, Sangen A, Wouters J, Desloovere C. Unilateral congenital hearing loss in children: Challenges and potentials. Hearing Research. 2019;372:29-41. DOI: 10.1016/j.heares.2018.01.010

[21] Lieu JE. Management of children with unilateral hearing loss. Otolaryngologic Clinics of North America. 2015;48(6):1011-1026

[22] Ross DS, Visser SN, Holstrum WJ, Qin T, Kenneson A. Highly variable population-based prevalence rates of unilateral hearing loss after the application of common case definitions. Ear and Hearing. 2010;31(1):126-313

[23] Lieu JE, Tye-Murray N, Fu Q. Longitudinal study of children with unilateral hearing loss. Laryngoscope. 2012;122:2088-2095

[24] Anne S, Lieu JEC, Cohen MS. Speech and language consequences of unilateral hearing loss: A systematic review. Otolaryngology and Head and Neck Surgery. 2017;157(4):572-579

[25] Koehlinger K, Van Horne AO, Oleson J, McCreery R, Moeller MP. The role of sentence position, allomorph, and morpheme type on accurate use of s-related morphemes by children who are hard of hearing. Journal of Speech, Language, and Hearing Research. 2015;58(2):396-409

[26] Sangen A, Royackers L, Desloovere C, Wouters J, van Wieringen A. Singlesided deafness affects language and auditory development-A case control study. Clinical Otolaryngology. 2017;42(5):979-987

[27] Krishnan LA, Van Hyfte S. Management of unilateral hearing loss. International Journal of Pediatric Otorhinolaryngology. 2016;88:63-73

[28] Arndt S, Aschendorff A, Laszig R, et al. Comparison of pseudobinaural hearing to real binaural hearing rehabilitation after cochlear implantation in patients with unilateral deafness and tinnitus. Otology $\&$ Neurotology. 2011;32:39-47

[29] Arndt S, Prosse S, Laszig R, Wesarg T, Aschendorff A, Hassepass F. Cochlear implantation in children with singlesided deafness: Does aetiology and duration of deafness matter? Audiology \& Neuro-Otology. 2015;20:21-30

[30] Kral A, O’Donoghue GM. Profound deafness in childhood. The New England Journal of Medicine. 2010;363(15):1438-1450

[31] Maslin MR, Munro KJ, El-Deredy W. Evidence for multiple mechanisms of cortical plasticity: A study of humans 
with late-onset profound unilateral deafness. Clinical Neurophysiology. 2013a;124(7):1414-1421

[32] Propst EJ, Greinwald JH, Schmithorst V. Neuroanatomic differences in children with unilateral sensorineural hearing loss detected using functional magnetic resonance imaging. Archives of Otolaryngology—Head \& Neck Surgery. 2010;136(1):22-26

[33] Vila PM, Lieu JE. Asymmetric and unilateral hearing loss in children. Cell and Tissue Research. 2015;361:271-278

[34] Kuppler K, Lewis M, Evans AK. A review of unilateral hearing loss and academic performance: Is it time to reassess traditional dogmata? International Journal of Pediatric Otorhinolaryngology. 2013;77:617-622

[35] Nuñez F, Jaúdenes C, Sequí, JM, Vivanco A, José Z. Diagnóstico y tratamiento precoz de la hipoacusia unilateral o asimétrica en la infancia: recomendaciones CODEPEH 2017. Revista Fiapas 2017; 163, Especial

[36] Kaplan AB, Kozin ED, Remenschneider A, Eftekhari K, Jung DH, Polley DB, et al. Amblyaudia: Review of pathophysiology, clinical presentation, and treatment of a new diagnosis, otolaryngology. Otolaryngology and Head and Neck Surgery. 2016 Feb;154(2):247-255

[37] Boyd PJ. Potential benefits from cochlear implantation of children with unilateral hearing loss. Cochlear Implants International. 2015;16(3):121-136. DOI: 10.1179/1754762814Y.0000000100

[38] Polonenko MJ, Papsin BC, Gordon KA. Children with single-sided deafness use their cochlear implant. Ear and Hearing. 2017;38(6):681-689. DOI: 10.1097/AUD.0000000000000452

[39] Ramos Macías A, Borkoski Barreiro S, Falcón González JC, Ramos de
Miguel A. AHL, SSD and bimodal CI results in children. European Annals of Otorhinolaryngology, Head and Neck Diseases. 2016;133(Suppl 1):S15-S20

[40] Van de Heyning P, Vermeire K, Diebl M, Nopp P, Anderson I, De Ridder D. Incapacitating unilateral tinnitus in single-sided deafness treated by cochlear implantation. The Annals of Otology, Rhinology, and Laryngology. 2008;117:645-652

[41] Vielsmeier V, Kreuzer PM, Haubner F, Steffens T, Semmler PR, Kleinjung T, et al. Speech comprehension difficulties in chronic tinnitus and its relation to hyperacusis. Frontiers in Aging Neuroscience. 2016;8:293

[42] Weidt S, Delsignore A, Meyer M, Rufer M, Peter N, Drabe N, et al. Which tinnitus-related characteristics affect current health-related quality of life and depression? A cross-sectional cohort study. Psychiatry Research. 2016;237:114-121

[43] Pan T, Tyler RS, Ji H, Coelho C, Gehringer AK, Gogel SA. Changes in the tinnitus handicap questionnaire after cochlear implantation. American Journal of Audiology. 2009;18:144-151

[44] Peters JP, van Zon A, Smit AL, van Zanten GA, de Wit GA, Stegeman I, et al. CINGLE-trial: Cochlear implantation for siNGLE-sided deafness, a randomised controlled trial and economic evaluation. BMC Ear, Nose and Throat Disorders. 2015;15:3

[45] Baguley DM, Atlas MD. Cochlear implants and tinnitus. Progress in Brain Research. 2007;166:347-355

[46] Arts RA, George EL, Janssen M, Griessner A, Zierhofer C, Stokroos RJ. Tinnitus suppression by intracochlear electrical stimulation in single sided deafness-A prospective clinical trial: Follow-up. PLoS ONE. 2016;11:e0153131 



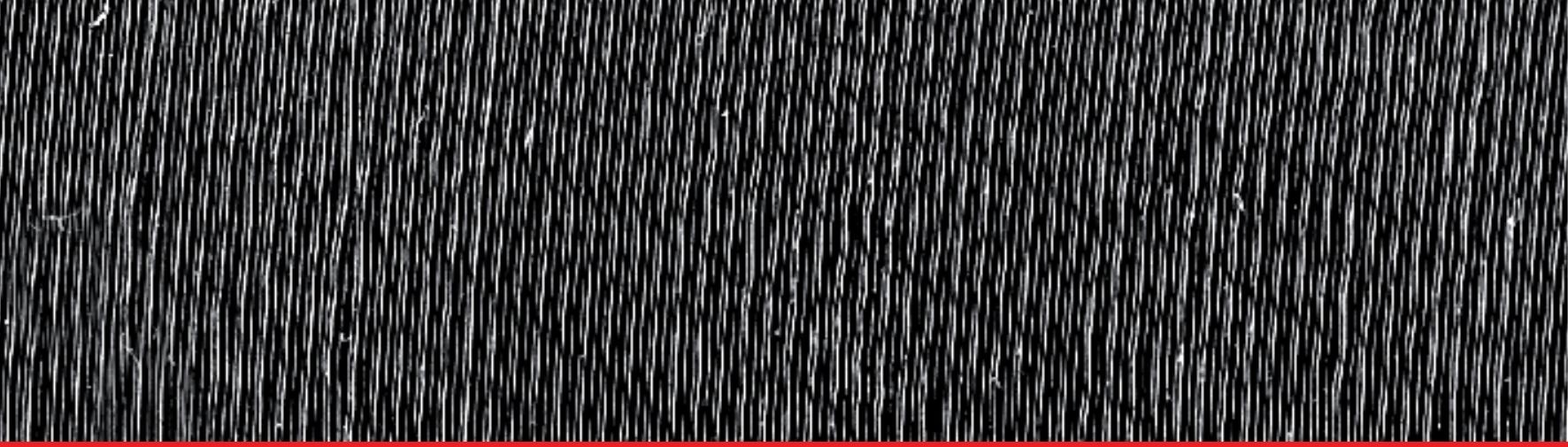

Edited by Diego Zanetti and Federica Di Berardino

This book covers some innovative aspects of the multifaceted and continuously evolving field of rehabilitation of hearing loss. International leading experts share their view and advanced experience on unilateral deafness, services for the hard of hearing,

hair cell regeneration, advanced imaging, active middle ear and bone conduction hearing aids, and cochlear implants.

Published in London, UK

๑ 2020 IntechOpen

๑ borzywoj / iStock

\section{IntechOpen}

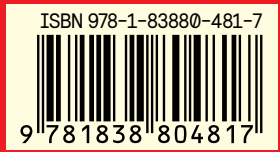

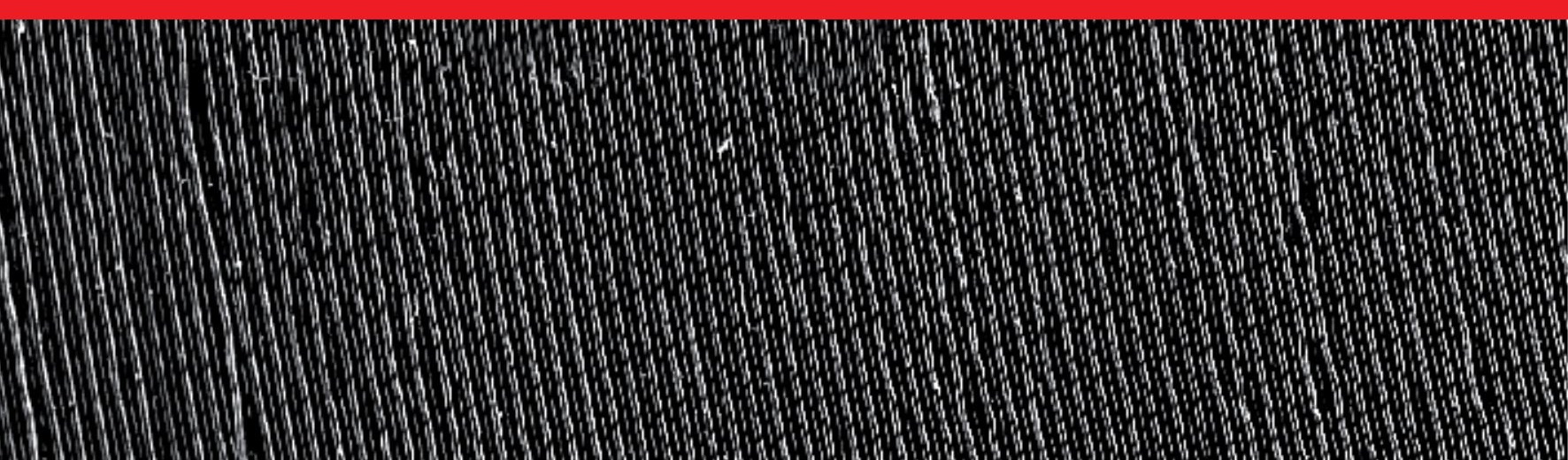

